\title{
Al1
}

Casa abierta al tiempo

UNIVERSIDAD AUTÓNOMA METROPOLITANA - IZTAPALAPA DIVISIÓN DE CIENCIAS BÁSICAS E INGENIERİA

\section{EFECTOS DE LAS INTERACCIONES \\ ELECTROSTÁTICAS SOBRE EL POTENCIAL EFECTIVO DE LÍQUIDOS MOLECULARES}

\author{
Tesis que presenta \\ Adrián Rocha Ichante \\ Para obtener el grado de \\ Doctor en Ciencias (Física)
}

Asesor: $\quad$ Dr Fernando del Río Haza

Jurado calificador:

Presidente: Dr Fernando del Río Haza

Secretario: Dr Antonio Moreno Razo

Vocal: Dr Enrique González Tovar

Vocal: Dr Mariano López de Haro

Vocal: Dr Andrés Estrada Alexanders

México, D. F. Noviembre 2014 
A Evelyn

A Leonardo 


\section{Agradecimientos}

Estas líneas las empleo para expresar mi más profundo y sincero agradecimiento a todas aquellas personas e instituciones que han colaborado de alguna u otra manera en la realización de este trabajo.

Agradezco a mi asesor el Dr Fernando del Río Haza por su dirección y supervisión. Su experiencia y entusiasmo han dejado un huella positiva en mi carrera. Siempre apreciaré su apoyo, confianza y sobre todo la paciencia que me ha tenido a lo largo de estos años.

A la Universidad Autónoma Metropolitana, en particular al Área de Líquidos de la Unidad Iztapalapa, por abrirme sus puertas, por facilitarme su infraestructura, y por darme la grata experiencia de formar parte de esta gran comunidad. Al CONACyT por apoyarme durante gran parte de esta etapa con un soporte económico tanto para la vida diaria como para lograr la movilidad a los distintos congresos a los que he asistido.

Al Dr Enrique Díaz Herrera y al Dr Antonio Moreno Razo por apoyarme en la parte computacional de esta investigación; su experiencia, programas y sugerencias han sido de gran valor.

Un reconocimiento especial les debo a mis amigos con los que conviví varios años en la UAM: Alicia, Alfredo, Benjamín, Gustavo, Rodolfo, y Tonalli. Todos esos momentos de debate, opinión y compañerismo siempre los tendré presentes.

Un agradecimiento muy intenso al ánimo constante que siempre me han regalado mis padres, hermanos y mi sobrino Roberto. A mi esposa Evelyn, por su cariño y comprensión, y a mi hijo Leonardo que, sin saberlo, me ha dado el impulso final para culminar esta etapa.

Muchas gracias. 


\section{Índice general}

\begin{tabular}{lc} 
Îndice de figuras & ix \\
\hline
\end{tabular}

Índice de tablas

Resumen xiii

\begin{tabular}{ll}
\hline 1. Introducción & 1
\end{tabular}

2. Elementos mecánico-estadísticos $\quad 5$

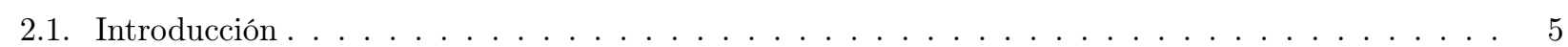

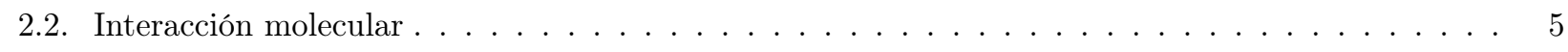

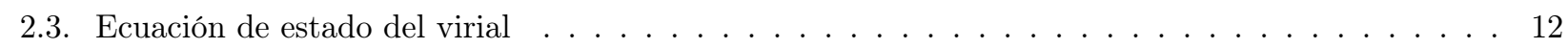

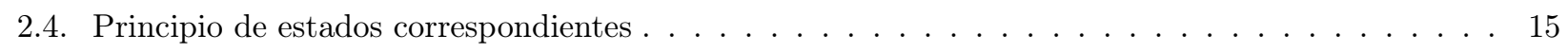

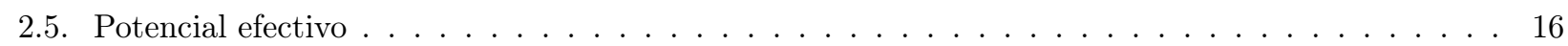

2.6. Simulación molecular . . . . . . . . . . . . . . . . . . . . . . . . . . 19

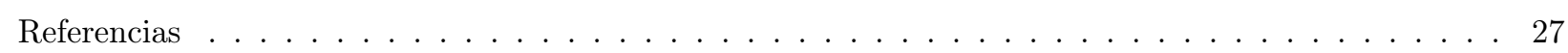

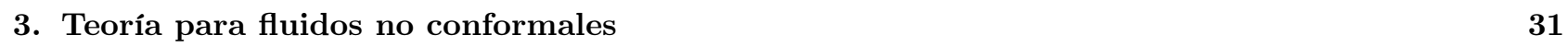

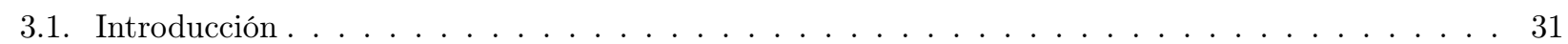

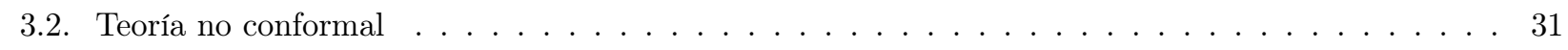

3.3. Teoría no conformal aproximada $\ldots \ldots \ldots \ldots \ldots \ldots \ldots \ldots$

3.4. El potencial ANC como modelo efectivo $\ldots \ldots \ldots \ldots \ldots \ldots \ldots$

3.5. Volúmenes de referencia ANC en forma analítica . . . . . . . . . . . . . . . . . 38

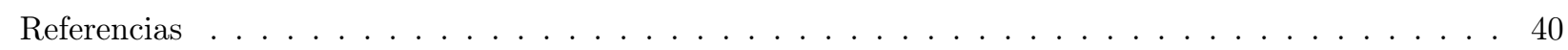

\begin{tabular}{|l|l}
\hline 4. Sistemas polares & 43
\end{tabular}

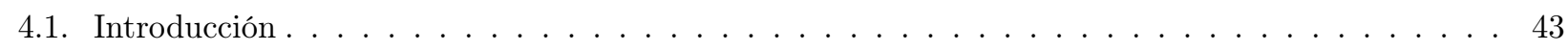

4.2. Antecedentes . . . . . . . . . . . . . . . . . . . . . . . . . . . 43

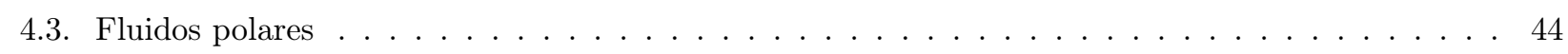

4.4. Sistema Stockmayer polarizable . . . . . . . . . . . . . . . . . . . . . 46

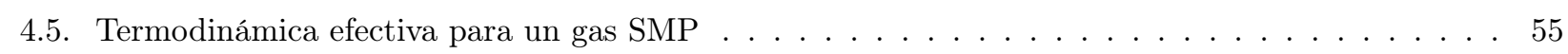

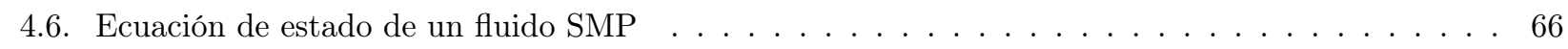

4.7. Simulación de sistemas SMP efectivos $\ldots \ldots \ldots \ldots$. . . . . . . . . . . . . . . . 73

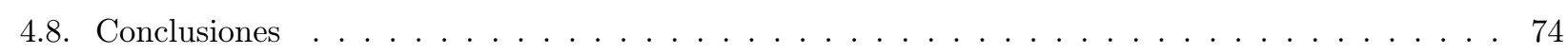

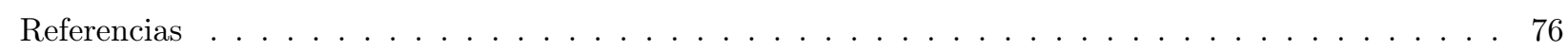




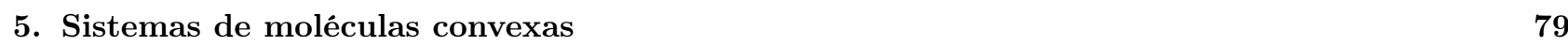

5.1. Introducción . . . . . . . . . . . . . . . . . . . . . . . . . . 79

5.2. Antecedentes . . . . . . . . . . . . . . . . . . . . . . . 80

$5.3 . \quad$ Pozos cuadrados convexos y la teoría ANC . . . . . . . . . . . . . . . . . . . . . . 80

5.4 . Potenciales ANC para moléculas convexas . . . . . . . . . . . . . . . . . . . . . . . 83

5.5. Aplicación a cuerpos convexos específicos . . . . . . . . . . . . . . . . . . . . . . . . . 88

5.6. Aplicación a sistemas reales . . . . . . . . . . . . . . . . . . . . . . . . 91

5.7. Conclusiones . . . . . . . . . . . . . . . . . . . . . . . . . . . . 93

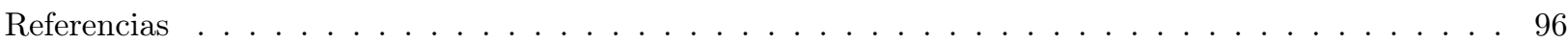

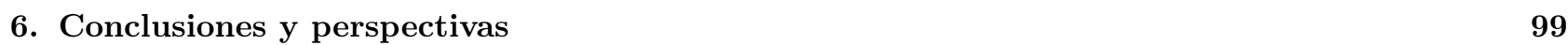

\begin{tabular}{|l|l}
\hline Apéndices & 103 \\
\hline
\end{tabular}

\begin{tabular}{|l|l|}
\hline A. Conceptos extras de Física Estadística & 105
\end{tabular}

A.1. Integrales de configuración . . . . . . . . . . . . . . . . . . . . . . . . . . . . 105

A.2. Expresiones analíticas de $B(T)$ y $C(T)$ para sistemas específicos . . . . . . . . . . . . . . . . . . 107

B. Integrales y ajustes numéricos extras.

B.1. Simplificación a la forma cerrada de $\mathcal{F}_{k}(T)$. . . . . . . . . . . . . . . . . . . . . . . . . . . . 111

B.2. Evaluación analítica de integrales . . . . . . . . . . . . . . . . . . . . . . . . . . 111

B.3. Ajustes numéricos . . . . . . . . . . . . . . . . . . . . . . . . . . . 115

\begin{tabular}{|l|l}
\hline C. Cuerpos no esféricos & 117 \\
\hline
\end{tabular}

C.1. Dinámica de cuerpo rígido . . . . . . . . . . . . . . . . . . . . . . . . . . . . . 117

C.2. Teoría básica de cuerpos convexos. . . . . . . . . . . . . . . . . . . . . . . . . . . 118

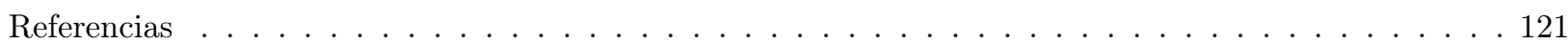




\section{Índice de figuras}

2.1. Diagrama PVT típico de un fluido real. $\ldots \ldots \ldots \ldots \ldots \ldots$

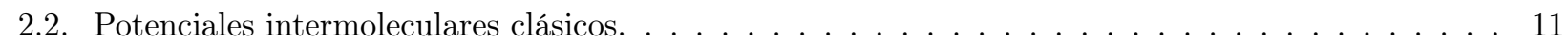

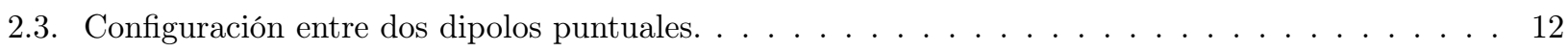

2.4. Segundo coeficiente virial de sustancias reales. . . . . . . . . . . . . . . . . . . . . 14

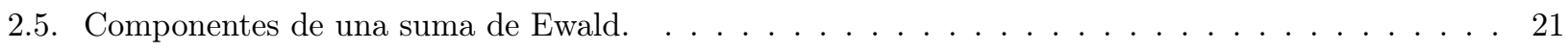

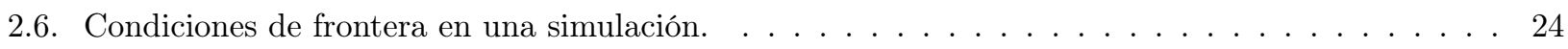

2.7. Lista de vecinos en una simulación de dinámica molecular simple. . . . . . . . . . . . . . 25

3.1. Comportamiento lineal ANC entre segundos coeficientes viriales. . . . . . . . . . . . . . . . 35

3.2. Cambio del potencial ANC con respecto a la suavidad. . . . . . . . . . . . . . . . 36

4.1. Efecto de la configuración entre dipolos sobre el potencial SMP . . . . . . . . . . . . . . . 47

$4.2 . \quad$ Efecto de la polarizabilidad en el potencial $\mathrm{SM} \ldots \ldots \ldots \ldots \ldots \ldots$. . . . . . . . . . 48

4.3. Dependencia de los potenciales SMP y SMPs con el momento dipolar. . . . . . . . . . . . . 52

4.4. Suavidades repulsiva y atractiva en función de la temperatura. $\ldots \ldots \ldots \ldots \ldots$

4.5. Suavidades repulsiva y atractiva en función del momento dipolar. . . . . . . . . . . . . . 55

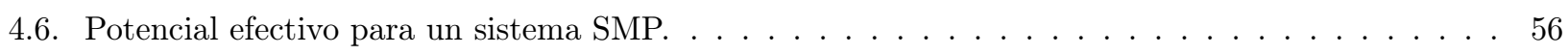

4.7. Energía y diámetro relativos para fluidos SMP con respecto a la temperatura y el momento

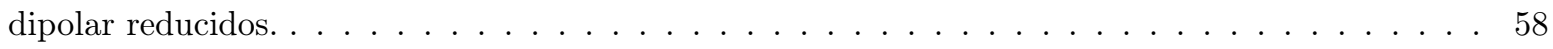

4.8. Predicción del segundo coeficiente virial efectivo para fluidos SMP. . . . . . . . . . . . . 61

4.9. Tercer coeficiente virial de un sistema SMP. . . . . . . . . . . . . . . . . . 62

4.10. Efecto de frustración en un sistema de tres dipolos. . . . . . . . . . . . . . . . 63

4.11. Contribución de los coeficientes viriales a la presión de un fluido SM. . . . . . . . . . . . 66

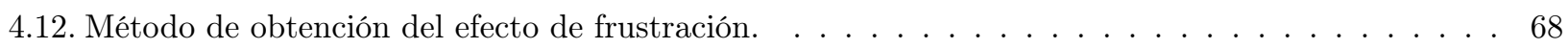

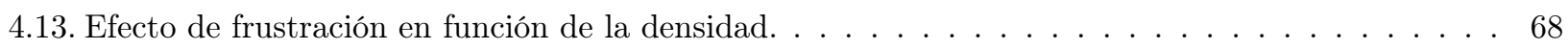

4.14. Temperatura crítica de un fluido SM en términos del momento dipolar. . . . . . . . . . . 70

4.15. Curvas ortobáricas para un fluido SM. Efecto de la frustración. . . . . . . . . . . . . . . 71

4.16. Curvas ortobáricas para un fluido SM. Efecto de la intensidad del dipolo. . . . . . . . . . . 72

4.17. Curvas ortobáricas para un fluido SMP. Efecto de la polarizabilidad. . . . . . . . . . . . . 72

4.18. Función de distribución radial mediante DM: de un fluido SM y de un fluido efectivo polar tipo LJ. 73

5.1. $B(T)$ para distintas elongaciones con respecto a $B_{0}(T) . \ldots \ldots \ldots \ldots \ldots$

5.2. Comportamiento lineal entre los coeficientes viriales con distintas elongaciones y la referencia

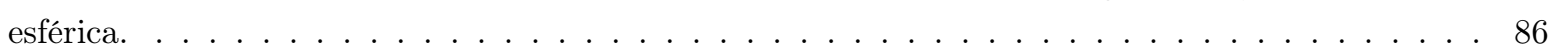

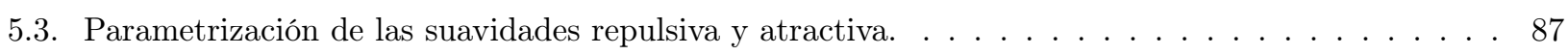

5.4. Segundos coeficientes viriales para interacciones CSW. . . . . . . . . . . . . . . . . . . . 89

5.5. Temperatura de Boyle y función suavidad para potenciales CSW. . . . . . . . . . . . . . 89 
5.6. Dependencia de los volúmenes de colisión con $T$ para una elongación dada. . . . . . . . . . . . . 90

5.7. Segundos coeficientes viriales para interacciones CANC. . . . . . . . . . . . . . . . . . . 91

5.8. Suavidades repulsiva y atractiva para interacciones CANC usando cuerpos convexos específicos. . 92

5.9. Función suavidad para alcanos y perfluoroalcanos. Dependencia con el número de carbonos. . . . 94

C.1. Cuerpo convexo formado por los centros de masa de dos cuerpos convexos. . . . . . . . . . . . . . 121 


\section{Indice de tablas}

4.1. Órdenes de contribución de la polarizabilidad al potencial SMP. . . . . . . . . . . . . . . . . . 46

4.2. Parámetros polares reducidos. . . . . . . . . . . . . . . . . . . . . . . . 50

4.3. Densidades de saturación. . . . . . . . . . . . . . . . . . . . . . . . . . . . . . . . . . . . . . . 69

5.1. Parámetros de ajuste para suavidades repulsiva y atractiva. . . . . . . . . . . . . . . . . . . . 86

5.2. Suavidades halladas para $n$-alcanos y $n$-perfluoroalcanos . . . . . . . . . . . . . . . . . . . 92

5.3. Ajustes para el modelo de suavidad única para un esferocilindro prolato. . . . . . . . . . . . . . . 93

B.1. Coeficientes de ajuste del parámetro de energía y distancia en un sistema polar. . . . . . . . . . . 115

B.2. Coeficientes de ajuste del parámetro de energía y distancia en un sistema polarizable. . . . . . . 115

C.1. Cuerpos convexos y sus medidas fundamentales. . . . . . . . . . . . . . . . . . . . . . 119

C.2. Cantidades características de algunos cuerpos convexos. . . . . . . . . . . . . . . . . . . . . 120 


\section{Resumen}

Esta tesis resume el trabajo realizado con potenciales efectivos sobre dos tipos de fluidos: el primero formado por moléculas que contienen dipolo eléctrico y el segundo con moléculas modeladas por cuerpos convexos. Los potenciales efectivos contienen un parámetro molecular extra, al de energía y al de distancia, que modifica el perfil del potencial con respecto a uno de referencia. El método empleado para ambos casos fue, esencialmente, trabajar con el promedio angular de las distintas configuraciones posibles entre dos moléculas y su posterior representación mediante un potencial efectivo simétricamente esférico; esto último se realiza mediante el principio de estados correspondientes. En el caso de moléculas convexas, el sistema efectivo representa adecuadamente el segundo coeficiente virial hallado por la termodinámica clásica de cuerpos convexos. En el caso polar, el potencial efectivo representa adecuadamente los primeros dos coeficientes viriales de un fluido teórico empleado regularmente en sistemas polares. Se ha incluido en dicho potencial un efecto de frustración energética entre dipolos lo cual ha permitido construir una ecuación de estado para este tipo de sistemas. 


\section{Capítulo 1}

\section{Introducción}

En la física estadística clásica a menudo se emplean modelos matemáticos, teóricos o empíricos, para obtener las propiedades termodinámicas de un fluido. El modelado puede hacerse en una o varias etapas del proceso de estudio y su complejidad dependerá del sistema que se analice y del enfoque empleado para ello. Desde un punto de vista fundamental, resultan importantes aquellos modelos que reproducen el potencial de interacción entre las moléculas de un sistema, ya que de esta forma pueden emplearse las distintas herramientas de la física estadística. Algunos de estos modelos de potencial engloban, en expresiones matemáticas sencillas, el comportamiento termodinámico y la estructura de sustancias simples, en especial a densidades bajas; el potencial Lennard-Jones es un buen ejemplo de ello. Desde entonces, una gran cantidad de ellos han sido construidos para representar el comportamiento de diversos grupos de sustancias reales a bajas y altas densidades, y generalmente se les denomina potenciales "realistas" ya que se supone reproducen el comportamiento de dichas sustancias con bastante exactitud.

Sin embargo, para la mayoría de los fluidos reales, el integrar en una sola expresión matemática todas las distintas interacciones moleculares que lo caracterizan hace que los potenciales sean poco manipulables a nivel teórico e incluso computacional. Si bien es cierto que en la actualidad las computadoras son lo suficientemente potentes para enfrentarse a modelos de tal complejidad, la existencia de una función mucho más sencilla que reproduzca aproximadamente la termodinámica del sistema original (aunque sea en un conjunto de estados específicos) implica una mejor comprensión del mismo desde un punto de vista físico. A estas nuevas funciones se les conoce como "potenciales efectivos" por su propiedad de reproducir de manera aproximada las características esenciales de un potencial realista, e incluso las de una sustancia real.

El éxito de un potencial efectivo al reproducir las propiedades termodinámicas del sistema de interés, aunado a su simplicidad y flexibilidad matemáticas, dependerá del ahorro teórico y/o computacional empleados en el proceso. Si su aplicación puede extenderse a un conjunto amplio de estados termodinámicos, e incluso a otros sistemas de similares características, sus ventajas se incrementarán sustancialmente. A pesar de que dicha efectividad esté limitada a una cantidad determinada de estados, su empleo aún es recomendable para trabajos muy puntuales en los que el modelo efectivo es muy exacto ó cuando se requieren resultados puramente cualitativos.

Generalmente, se busca que un potencial efectivo contenga parámetros vinculados con características moleculares del sistema analizado. En este contexto el potencial efectivo es un concepto altamente ligado al principio de estados correspondientes a nivel molecular; ésto se debe a que dicho principio utiliza una función "universal" que reproduce las interacciones moleculares de un conjunto de sistemas a los que comúnmente se les llama conformales.

En este trabajo se ha desarrollado el concepto de potencial efectivo y es aplicado a sistemas formados tanto por moléculas polares como convexas; dicho estudio ha sido dirigido principalmente a estados diluidos, aunque para el caso de sistemas polares se consideran incluso altas densidades. Todo se construye alrededor de un tipo 
de partículas que siguen el comportamiento dado por un potencial efectivo llamado ANC, y que ha sido aplicado en los últimos años para calcular de manera muy precisa una variedad de propiedades de sustancias simples $\mathrm{y}$ sus mezclas. La teoría que fundamenta esta función (llamada precisamente ANC) suele considerarse como una extensión al principio de estados correspondientes ya que lo generaliza para sistemas no conformales utilizando un parámetro extra llamado suavidad el cual controla la conformalidad entre dos potenciales. El potencial ANC para fluidos reales contiene parámetros de interacción dependientes de las características moleculares de la sustancia de interés; la polarizabilidad, los momentos electrostáticos y la elongación de la moléculas serán algunas de las características aquí presentes.

El orden de la presente tesis es el siguiente: en el capítulo 2 se tratan de forma breve aquellos temas que recurrentemente aparecen en este trabajo. Se introduce el concepto de interacción molecular y la manera en la que comúnmente se clasifican para su estudio. Posteriormente, se incluyen algunos modelos matemáticos simples, ya clásicos en el ámbito de la física de fluidos, que representan las principales interacciones moleculares empleadas en el presente estudio. En el mismo capítulo se presenta el principio de estados correspondientes y su importancia en el estudio de fluidos diluidos conformales. En seguida se realiza una breve introducción a la teoría de potenciales efectivos y su extensión para estudiar sistemas más complejos. Al final de este capítulo se hace una pequeña revisión sobre cómo simular fluidos mediante la dinámica molecular; se tocan los principales aspectos para sistemas simples y para aquellos sistemas que incluyen interacciones del tipo polar.

El capítulo 3 se destina por completo a presentar los fundamentos básicos de la teoría ANC, así como las principales ventajas en su aplicación a sistemas fluidos. Se pone atención especial al concepto de "suavidad" y su inclusión como un parámetro molecular extra en la función potencial ANC. Ese parámetro determina de manera rigurosa que el segundo coeficiente virial de cualquier sustancia ANC puede escribirse en términos del respectivo coeficiente de un sistema de referencia; dicha funcionalidad es lineal y sencilla. Una demostración de dicha relación, para el caso más general, ha sido obtenida como parte de este trabajo, aportando expresiones analíticas para este coeficiente virial y todas ellas han sido empleadas en este trabajo. La visión ANC para el tercer coeficiente virial, y su comportamiento en la zona líquida y otras propiedades termodinámicas ha rendido excelentes resultados para distintos sistemas y ellos son comentados al final del capítulo.

En el capítulo 4 se presenta la investigación realizada sobre los sistemas polares y polarizables, donde se han construido potenciales efectivos que reproducen fluidos cuyas partículas son dipolos puntuales. El potencial Stockmayer, junto con sus variantes, han sido empleados repetidamente en los últimos años por su excelente capacidad para reproducir los efectos electrostáticos a nivel gas y líquido de fluidos reales; dichas interacciones son, a pesar de su simplicidad frente a otros modelos, difíciles de manejar en especial a densidades altas donde la simulación computacional es necesaria. En este capítulo se muestra como un potencial efectivo tipo LJ funciona adecuadamente para los sistemas Stockmayer a bajas densidades y momentos dipolares pequeños; para momentos dipolares mayores un potencial tipo ANC reproduce de manera efectiva a este tipo de sistemas, inclusive al polarizable. En particular, los primeros coeficientes viriales de este tipo de sistemas teóricos son conocidos de manera analítica y esta información ha servido para verificar la eficacia de la propuesta efectiva.

En este mismo capítulo se extienden los resultados obtenidos en la fase gaseosa para densidades mayores; en específico, se trabaja con el tercer coeficiente virial y el equilibrio líquido-vapor. Esto se logra de forma efectiva al introducir un efecto de "frustración energética" generado por la presencia de un dipolo mientras interactúan otros dos de ellos. Este factor permite que el tercer coeficiente virial presente el comportamiento adecuado con respecto a la temperatura, además de servir como medio (empleando resultados teóricos y simulados de otros autores) para construir una ecuación de estado para temperaturas subcríticas y momentos dipolares reducidos de intensidad media. Posteriormente, se construyen las curvas ortobáricas respectivas, se identifican los parámetros críticos y se muestra la compatibilidad de dicha ecuación, al incluir la polarizabilidad, con resultados de simulación de otras investigaciones.

Los resultados que surgen del estudio de potenciales efectivos (especialmente en fases densas para sistemas dipolares) son respaldados con datos obtenidos vía la simulación molecular. Se han realizado algunas 
simulaciones de sistemas electrostáticos y los resultados son comparados con la simulación simple de sistemas efectivos en las secciones finales de este capítulo. La parte final del capítulo se dedica a las conclusiones de este tema y cuyos resultados han sido reportados en un artículo científico de una revista de alto impacto y prestigio. Este artículo se anexa como complemento al final de la tesis.

La investigación orientada a sistemas en fase diluida formados por cuerpos convexos son contemplados en el capítulo 5 Los conceptos básicos de la teoría de cuerpos convexos y una breve introducción a la termodinámica de este tipo de sistemas ha sido revisada a lo largo de la tesis. En esta parte del trabajo toda esa teoría es revisada desde el marco ANC con parámetros moleculares escritos en términos de las medidas fundamentales del cuerpo convexo utilizado. Primero se estudian moléculas convexas que interaccionan mediante un potencial de pozo cuadrado convexo, obteniéndose con ello los volúmenes de colisión, propios de la teoría ANC, así como el segundo coeficiente virial del fluido en función de la elongación efectiva de la molécula.

La segunda parte de este capítulo se concentra en la generalización de los conceptos anteriores y en la construcción de un potencial continuo entre moléculas convexas. El potencial esférico generado se supone con características similares a la de un potencial tipo Kihara por lo que las definiciones de los volúmenes ANC de colisión tienen una dependencia única con la distancia internodular. La solución analítica de estos volúmenes es similar a la que caracteriza a la teoría ANC permitiendo identificar de forma exacta las suavidades repulsiva y atractiva en términos de las medidas fundamentales del cuerpo convexo empleado para ello. La aplicación de estas relaciones para algunos cuerpos en particular, como los esferocilindros y los elipsoides, sirve de base para relacionar la elongación efectiva de una molécula modelo con moléculas cuasilineales reales tales como los alcanos y perfluoroalcanos, cuyos parámetros ANC ya son conocidos. El capítulo finaliza con la presentación de las principales observaciones y conclusiones que se obtienen utilizando un potencial efectivo para estas dos familias de sustancias reales.

Una recapitulación final del trabajo se hace en el capítulo 6. En él se presentan las conclusiones más importantes de la investigación, y a partir de un análisis breve de ellas se plantean nuevas interrogantes y perspectivas a corto y largo plazo con respecto a los temas aquí abordados. 


\section{Capítulo 2}

\section{Elementos mecánico-estadísticos}

\subsection{Introducción}

En este capítulo se presentan los principales aspectos mecánico-estadísticos que aquí se usan continuamente. Se inicia con una breve reseña acerca de la naturaleza de un fluido real simple y el posible estudio de su estado termodinámico mediante el uso de una ecuación de estado. Desde un marco teórico dicha ecuación requiere información que indique cómo interactúan las moléculas que componen a ese fluido y también, en gran medida, de una expresión matemática que englobe toda esa información de forma conveniente. En la sección 2.2 se introduce el concepto de interacción molecular y se presenta una forma habitual de clasificar las interacciones en términos del alcance energético. Esta caracterización puede condensarse en modelos sencillos los cuales son ampliamente conocidos en el estudio de líquidos y en esta parte se presentan algunos de ellos. Estos modelos intermoleculares permiten generar la termodinámica de un sistema mediante la teoría del desarrollo del virial por lo que la sección 2.3 resume las principales características de la ecuación de estado surgida de este desarrollo escrita en términos de los llamados coeficientes viriales; nos enfocamos principalmente en los dos primeros coeficientes debido a que el estudio de la fase gaseosa forma parte primordial en esta investigación.

La representación de la interacción molecular mediante un modelo matemático sencillo lleva a la noción de potencial efectivo y el fundamento teórico que lo sustenta. Desde esta perspectiva se maneja el principio de estados correspondientes en la sección 2.4, tanto para sistemas simples como su generalización para sistemas más complejos, con el fin de manejar una función potencial única que represente un conjunto de sustancias reales. Este potencial que de manera "efectiva" reproduce la propiedad termodinámica de un conjunto de sustancias reales es introducido en la sección 2.5.

\subsection{Interacción molecular}

\subsubsection{Introducción}

Una ecuación de estado (EDE) es una relación funcional entre las variables termodinámicas intensivas de un sistema. Ésta reduce los grados de libertad independientes necesarios para describir el estado de dicho sistema [1-3]. En el caso de que el sistema sea una sustancia pura compresible, el estado puede definirse por tres propiedades, dos de ellas independientes, y ligadas por la respectiva EDE. Por su facilidad de manejo se emplean las propiedades de presión $(P)$, volumen $(V)$ y temperatura $(T)$. A partir de la EDE puede obtenerse una variable partiendo de las otras dos, además de propiedades que no pueden medirse directamente tal como la energía y la entropía que dependen del sistema [4]. La EDE más simple es la llamada "ideal" que esta dada por

$$
P V=N k_{\mathrm{B}} T,
$$


donde $k_{\mathrm{B}}$ es la constante de Boltzmann y $N$ es el número de partículas que componen al sistema; al sistema que cumple esta ecuación recibe también el nombre de ideal. Desde un punto de vista molecular las partículas que forman al sistema ideal no interactúan entre sí, aunque para muchas sustancias reales esta ecuación puede ser empleada de forma aproximada. Estos casos se dan para densidades lo suficientemente bajas en los que un número pequeño de las moléculas que la componen interactúan simultáneamente sin verse afectadas por el resto de las otras. Resulta claro que a medida que aumenta la densidad del sistema las interacciones reales entre sus moléculas cobran mayor relevancia por lo que la EDE ideal deja rápidamente de representar su estado termodinámico.

Una manera usual de medir cuánto se desvía el comportamiento de un sistema real (en un estado dado) con respecto al ideal es mediante el factor de compresibilidad

$$
Z \equiv \frac{P V}{N k_{\mathrm{B}} T} ;
$$

dicho factor tomará el valor de la unidad para un gas ideal teórico y en el caso de gases reales muy diluidos, la EDE ideal es ocupada con gran exactitud. Además, la experiencia muestra que cualquier gas real para el cual la presión (ó la temperatura) tiende a cero también tiende a cumplir un comportamiento ideal [3-5].

El comportamiento físico de un fluido en términos del estado del sistema puede entenderse con la ayuda de un diagrama $P-V$-T. La figura 2.1 muestra un ejemplo típico de las isotermas de un fluido en función de las propiedades termodinámicas restantes. Las isotermas hiperbólicas equiláteras son características de un gas ideal, de tal forma que cuando la temperatura disminuye la hipérbola comienza a descomponerse indicando un alejamiento de la idealidad del gas que se estudia.

Aquella isoterma caracterizada por un punto de inflexión con pendiente horizontal es muy importante en el estudio de un fluido; al estado que identifica a dicho punto y a dicha isoterma se les conoce comos críticos y a las cantidades $P_{\mathrm{c}}, V_{\mathrm{c}}$ y $T_{\mathrm{c}}$ se denominan constantes críticas [3,6]. Al realizar la compresión de dicho gas a la temperatura crítica $T_{\mathrm{c}}$ constante no aparece superficie de separación de dos fases ${ }^{1}$ La isoterma crítica separa los estados de un fluido en los que la sustancia estará siempre en una fase de la de aquellos que pueden dividirse en dos.

Por debajo de $T_{\mathrm{c}}$ puede lograrse la condensación a la fase líquida. Para analizar dicho cambio puede tomarse cualquier isoterma de la figura 2.1 siguiendo el comportamiento real del gas a medida que éste se comprime. Así, cerca del estado I, y aumentando la presión del gas se cumple de forma aproximada la ley de gas ideal, ec. 2.1. No obstante, comienzan a aparecer desviaciones de la ley cuando el volumen se ha reducido hasta el estado II y cuando se llega al punto III, toda relación con el gas ideal ha desaparecido. A partir de III, aunque el volumen del sistema disminuye, la presión no aumenta. Es en esa zona donde comienza a aparecer el líquido con una separación visible entre fases y cuanto más disminuye el volumen más líquido aparece. La presión correspondiente a este intervalo de estados (línea horizontal III-IV-V) se le denomina presión de vapor del líquido para la temperatura que define la isoterma ${ }^{2}$ Cuando se alcanza el estado VI la muestra es completamente líquida y cualquier nueva reducción de volumen requerirá una presión alta como se observa en la misma curva. Analíticamente, tal comportamiento termodinámico de la sustancia real puede estudiarse de forma adecuada mediante el uso de una EDE conveniente. La EDE permite estudiar zonas termodinámicas específicas tales como la crítica; por ejemplo, se tiene que la isoterma crítica pasa a través de un punto de inflexión (el punto crítico) el cual indica que el cambio en la presión (cantidad obtenida vía una EDE) con respecto al volumen cumple las siguientes dos condiciones matemáticas [3]:

$$
\left(\frac{\partial P}{\partial V}\right)_{T=T_{\mathrm{c}}}=0,
$$

\footnotetext{
${ }^{1}$ A una temperatura $T>T_{\mathrm{c}}$ la sustancia consiste en una única fase (que por definición es un gas) que ocupa el volumen completo del recipiente que la contiene impidiendo la formación de la fase líquida. Debido a que esta fase puede ser mucho más densa de la que regularmente se tiene para el gas se le da el nombre de fluido supercrítico [3].

${ }^{2}$ Los términos vapor saturado y líquido saturado suelen utilizarse para referirse al gas y el líquido en equilibrio mútuo.
} 


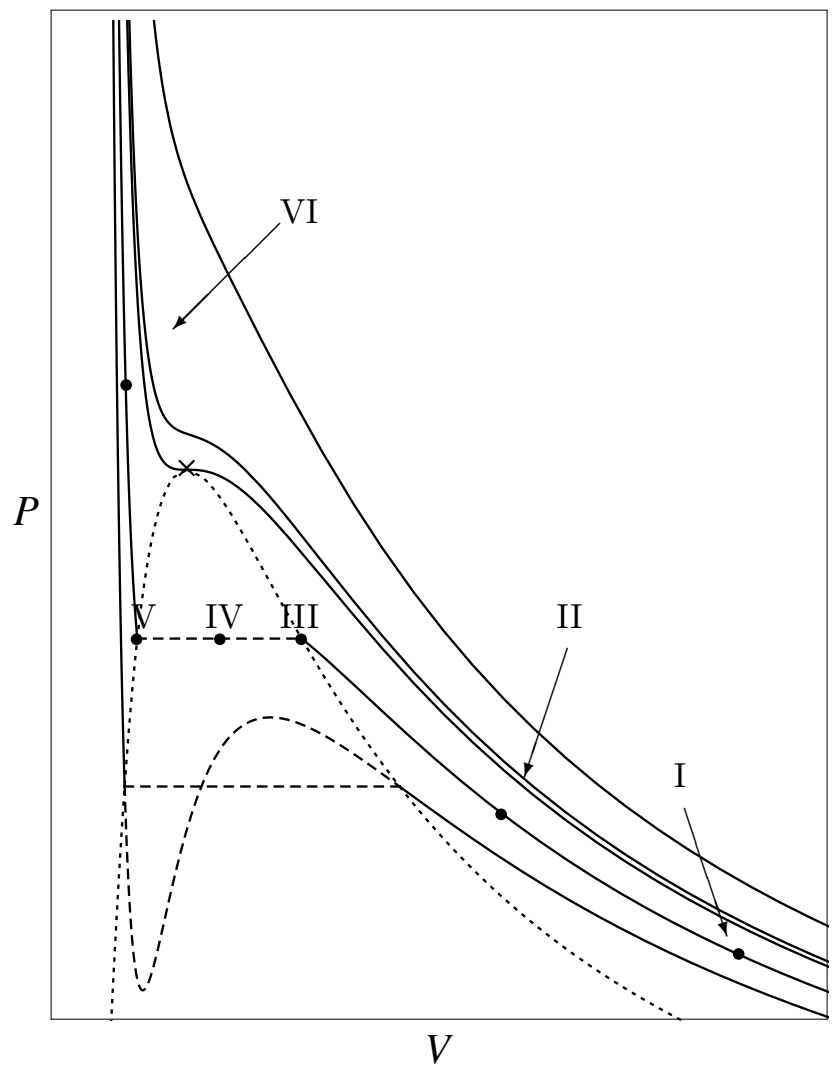

Figura 2.1: Diagrama PVT típico de un fluido real. La región por debajo de la línea punteada representa una zona de dos fases en equilibrio e indica los límites de la coexistencia. El punto crítico (marcado con un asterisco) se encuentra en el máximo de esta curva. El bucle (de van der Waals) a trazos muestra el comportamiento de algunas EDE (entre ellas las cúbicas) en la región de dos fases.

y

$$
\left(\frac{\partial^{2} P}{\partial V^{2}}\right)_{T=T_{\mathrm{c}}}=0
$$

es decir, para localizar el punto crítico de una sustancia se debe utilizar una EDE adecuada a ella y se resuelven (2.2) y 2.3) simultáneamente para encontrar las propiedades críticas correspondientes.

La condición 2.2, indica que si en cierto espacio la densidad de partículas se modifica no surge una presión que se contraponga a tal efecto lo que provoca que en el estado crítico las fluctuaciones de la densidad se hagan muy grandes [4], por lo que el índice de refracción y absorción del medio varía intensamente de un punto a otro. Por ello, la luz se disipa drásticamente y se dispersa en el medio lo que conduce al fenómeno conocido como opalescencia crítica; además, las fluctuaciones críticas son independientes de la constitución molecular, así que cerca de sus puntos críticos todos los fluidos tienen características en común.

Existen muchas propuestas de EDE que varían en forma y en número de parámetros, y su aplicación estará en función de la exactitud que se requiera y de la región termodinámica en la cual se trabaje. Entre las EDE más estudiadas se encuentran las que tienen sólo dos parámetros; uno de ellos mide la cohesión entre las moléculas y el otro es proporcional a su volumen, y suelen ser representadas por las letras $a$ y $b$, respectivamente. La EDE de este tipo más conocida es la de van der Waals

$$
\left(P+\frac{N^{2} a}{V^{2}}\right)(V-N b)=N k_{\mathrm{B}} T
$$

cuya forma permite ser reescrita como una ecuación cúbica con respecto al volumen (o la densidad) y con solución analítica: los parámetros de la EDE para una sustancia en particular, pueden determinarse a partir de las respectivas constantes críticas usando las condiciones (2.2) y (2.3) $3^{3}$ A partir de la forma de esta EDE

\footnotetext{
${ }^{3}$ En realidad esta no es una característica exclusiva de esta ecuación. Cualquier EDE de 2 o 3 parámetros con punto crítico lo permite.
} 
se han desprendido un gran número de variantes $4^{4}$ las cuales reproducen de manera más o menos acertada el comportamiento cualitativo de un gas real con una elección adecuada de sus parámetros en una región limitada del diagrama $P-V-T$.

Una visión con mayor fundamento teórico en el estudio de un fluido implica conocer cómo interactúan entre sí las partículas que lo forman, incluyendo todas aquellas interacciones con el exterior. Tal caracterización se puede lograr de forma completa al resolver la ecuación de Schrödinger de tal sistema adoptando algunas aproximaciones [8]. En general, el potencial de interacción de todas las partículas del sistema que arroja tal método puede ser escrito en términos de interacciones entre partículas de manera individual, por pares, triadas, etc., lo que se representa formalmente comq $5[9]$

$$
\Phi\left(\mathbf{r}_{1}, \cdots, \mathbf{r}_{N} ; \Omega\right)=\sum_{i} \Phi_{1}\left(\mathbf{r}_{i}\right)+\sum_{i<j} \Phi_{2}\left(\mathbf{r}_{i}, \mathbf{r}_{j}\right)+\sum_{i<j<k} \Phi_{3}\left(\mathbf{r}_{i}, \mathbf{r}_{j}, \mathbf{r}_{k}\right)+\cdots+\Phi_{N}\left(\mathbf{r}_{1}, \cdots, \mathbf{r}_{N}\right)
$$

donde $\mathbf{r}_{i}$ es la posición de la $i$-ésima partícula y la inclusión del símbolo $\Omega$ en el lado izquierdo de la ecuación significa que existe una dependencia orientacional implícita entre las partículas para cada una de las contribuciones del lado derecho 6

Efectivamente, si se tuviera un conocimiento completo de cada uno de los términos de la ecuación anterior se obtendría una descripción microscópica plena de la sustancia en estudio. Sin embargo, sólo a un nivel cuántico han sido obtenidos resultados cuantitativos para modelos simples e ideales lo que hace poco práctica su implementación a nivel macroscópico mediante el uso de la física estadística. Históricamente, el estudio del potencial intermolecular se ha concentrado esencialmente en el segundo término de la derecha de 2.5 que involucra a dos de las partículas del sistema. Esta situación es mucho más marcada para el fluido diluido donde los últimos términos del lado derecho tienen menor relevancia respecto a los primeros:7 A este enfoque se le conoce como aditividad (o superposición) por pares el cual considera solamente al segundo término del lado derecho de la ec. 2.5 es decir

$$
\Phi\left(\mathbf{r}_{1}, \cdots, \mathbf{r}_{N} ; \Omega\right) \approx \sum_{i, j} \Phi_{2}\left(\mathbf{r}_{i}, \mathbf{r}_{j}\right)
$$

En general, un conocimiento apropiado de las interacciones $\Phi_{2}$ ha sido esencial en el estudio de los líquidos y sus propiedades termodinámicas; debido a esto se ha generado un gran número de modelos de interacciones binarias, que aunque limitados en algunos casos, dan una base cualitativa o semicuantitativa para comprender y correlacionar resultados experimentales.

\subsubsection{Energía intermolecular y su clasificación}

El estudio del comportamiento termodinámico de las sustancias (principalmente en fase gaseosa) ha llevado a clasificaciones diversas de las energías intermoleculares, considerando las características principales de las moléculas que las componen. La energía de interacción entre un par de moléculas puede dividirse en energías de corto y de largo alcance $[10-12]]^{8}$

\footnotetext{
${ }^{4}$ Aunque la EDE de van der Waals ha tenido un peso importante en el estudio de los fluidos, en virtud de que fue la primera que predijo la transición líquido-vapor, cuantitativamente se toma como un punto de partida para encontrar mejores ecuaciones de estado empíricas postulando condiciones adecuadas en la frontera en los límites de baja y alta densidad. La implementación de este tipo de condiciones estimulan nuevas mejoras en el desarrollo de EDE cúbicas analíticas. Una revisión completa y reciente sobre EDE cúbicas se presentan en el capítulo 4 de la referencia [7].

${ }^{5}$ Nótese que esta definición no impone restricción alguna sobre el número de especies en el sistema.

${ }^{6} \mathrm{El}$ primer término del lado derecho de 2.5 se hace cero en el caso en que se consideren partículas sin estructura o no existan interacciones externas sobre las mismas.

${ }^{7} \mathrm{El}$ hecho de emplear únicamente la interacción entre un sólo par de moléculas y que muchas veces concentre la física completa del sistema estudiado es el objetivo planteado por un potencial efectivo, concepto que se tocará más adelante.

${ }^{8}$ Aunada a este tipo de energía se pueden incluir las de alcance intermedio las cuáles tiene su origen en los términos de segundo orden de las energías de intercambio. A distancias intermedias su importancia radica en evitar la suma directa entre los términos de corto y largo alcance $[9,13]$.
} 


\section{Energía de corto alcance}

El origen de este tipo de interacciones es cuántico y surge (cuando dos moléculas se acercan lo suficiente) al traslape entre sus nubes electrónicas. El principio de exclusión de Pauli prohibe que dos electrones ocupen un mismo estado cuántico lo que deriva en un efecto de repulsión. Las energías en este intervalo pueden obtenerse vía teoría de perturbación de primer orden y se les conoce como energías de intercambio de primer orden; la expresión más simple que la representa es [13]

$$
\Phi_{2}=A e^{-B r}
$$

donde $A$ y $B$ son constantes y $r$ es la distancia entre los centros de masa. Algunas veces dicha energía puede ser representada por una expresión proporcional a una potencia inversa de la distancia entre los centros de las moléculas [14]

$$
\Phi_{2}=\frac{\nu}{r^{n}}
$$

donde $n$ y $\nu$ son constantes.

\section{Energías de largo alcance}

Estas energías tienen un origen electromagnético y pueden dividirse, a su vez, en tres tipos: a) electrostáticas, b) de inducción y c) de dispersión $[10,13]$. Las últimas dos requieren una base mecánicocuántica para su comprensión.

\section{a) Electrostática ${ }^{9}$}

En general, la distribución electrónica en una molécula puede expresarse como un desarrollo multipolar [15]; la energía electrostática surge así de la interacción entre los diversos multipolos de las moléculas con una dependencia en sus intensidades ya sean de la carga, dipolo, cuadripolo, etc. En el caso unipolar la energía entre dos cargas viene dada por la relación clásica de Coulomb; es decir, proporcional a la inversa de la distancia. Para el caso de moléculas neutras, y con una distribución de carga asimétrica, el primer término interactivo se da entre dos dipolos permanentes con una energía proporcional a $r^{-3}$ y al producto entre las magnitudes de los dipolos ${ }^{10}$ En el mismo orden, desde un punto de vista perturbativo 11 la presencia de una molécula extra no produce ninguna distorsión en la distribución electrónica del par de moléculas involucradas; por lo tanto, las distintas interacciones de este punto se consideran aditivas para cada par de moléculas.

\section{b) de inducción}

Este tipo de energía surge de la polarización de una molécula por otra y siempre tiene una importancia secundaria respecto a las interacciones electrostáticas permanentes. A esta propiedad que tiene la molécula de ser polarizada se le conoce como polarizabilidad. Parte de la generación de dipolos inducidos contribuyen energéticamente ya sea al interaccionar con el resto de los multipolos permanentes o con otro dipolo inducido. Estas energías no son aditivas ya que surgen debido a la presencia de una tercer molécula; mientras más cerca se encuentre ésta al par de estudio mayor será la energía inductiva del sistema.

\section{c) de dispersión}

La contribución de este tipo de interacción proviene tanto de moléculas no-polares como polares y su explicación formal se hace en términos de la mecánica cuántica ${ }^{12}$ De manera semiclásica estas energías pueden entenderse como surgidas de la inducción generada por dipolos instantáneos entre

\footnotetext{
${ }^{9} \mathrm{~A}$ menudo se le conoce como contribución orientacional debido a que la configuración entre moléculas aparece explícitamente en su forma funcional.

${ }^{10}$ Una revisión más amplia, desde un enfoque efectivo, será realizada cuando se toque este tipo de sistemas en el capítulo 4

${ }^{11} \mathrm{La}$ inducción electrostática en una molécula generada por otra corresponde al segundo orden perturbativo.

${ }^{12}$ También conocida como energía de dispersión de London.
} 
las moléculas. En promedio se genera una atracción entre moléculas expresada como una serie del tipo

$$
\Phi_{\text {disp }}=-\frac{c_{1}}{r^{6}}-\frac{c_{2}}{r^{8}}-\frac{c_{3}}{r^{10}}-\cdots
$$

donde $c_{1}, c_{2}, c_{3}$, etc, son constantes positivas y su valor dependerá de la molécula de estudio.

\subsubsection{Modelos clásicos de interacción molecular}

Existen diversos modelos que representan en mayor o menor medida la interacción específica existente entre las moléculas de una sustancia (o un grupo característico de ellas); sin embargo, algunos de los más básicos utilizados en el estudio de líquidos se muestran a continuación [8,16]. En particular, se presta mayor atención en aquellos modelos que son utilizados en este trabajo y el comportamiento de todos ellos se presentan en la figura 2.2 .

\section{Esfera dura}

Este potencial representa a esferas duras (HS) de diámetro $\sigma$ y cuya interacción está dada por la siguiente forma funcional

$$
\Phi_{\mathrm{HS}}(r)=\left\{\begin{array}{cc}
\infty, & r \leq \sigma \\
0, & r>\sigma
\end{array} ;\right.
$$

lo que indica que dos moléculas no interactúan hasta el momento en que tienen contacto; un punto importante en este potencial es que no existen fuerzas atractivas entre las moléculas.

\section{Pozo cuadrado}

El potencial de pozo cuadrado (SW) es una extensión directa del modelo anterior al incluir energías atractivas y está formado por un nódulo esférico repulsivo rodeado por un cascarón atractivo también esférico; dicho espacio entre el nódulo y el cascarón (el pozo) tiene una profundidad energética $-\epsilon$. Los tamaños del nódulo y el cascarón suelen escribirse proporcionales al diámetro del nódulo central $\sigma$. La interacción entre dos moléculas SW está dada por

$$
\Phi_{\mathrm{SW}}(r)=\left\{\begin{array}{cc}
\infty, & r \leq \sigma, \\
-\epsilon, & \sigma<r \leq \lambda \sigma, \\
0, & \lambda \sigma<r,
\end{array}\right.
$$

donde $\sigma$ es el ancho del nódulo duro y $\lambda$ es un parámetro de escala que mide el ancho del pozo.

\section{Lennard-Jones 12-6}

La interacción tipo Lennard-Jones (LJ) tiene la forma

$$
\Phi_{\mathrm{LJ}}(r ; \delta, \epsilon)=\epsilon\left[\left(\frac{\delta}{r}\right)^{12}-2\left(\frac{\delta}{r}\right)^{6}\right]
$$

donde $\delta$ y $\epsilon$, son la posición y energía del mínimo del potencial, que cumplen $\Phi_{\mathrm{LJ}}(r=\delta)=-\epsilon$. Cuando se usa el parámetro de distancia $\sigma$, para el cual $\Phi_{\mathrm{LJ}}(r=\sigma)=0$, se obtiene una forma alterna para este potencial

$$
\Phi_{\mathrm{LJ}}(r ; \sigma, \epsilon)=4 \epsilon\left[\left(\frac{\sigma}{r}\right)^{12}-\left(\frac{\sigma}{r}\right)^{6}\right]
$$




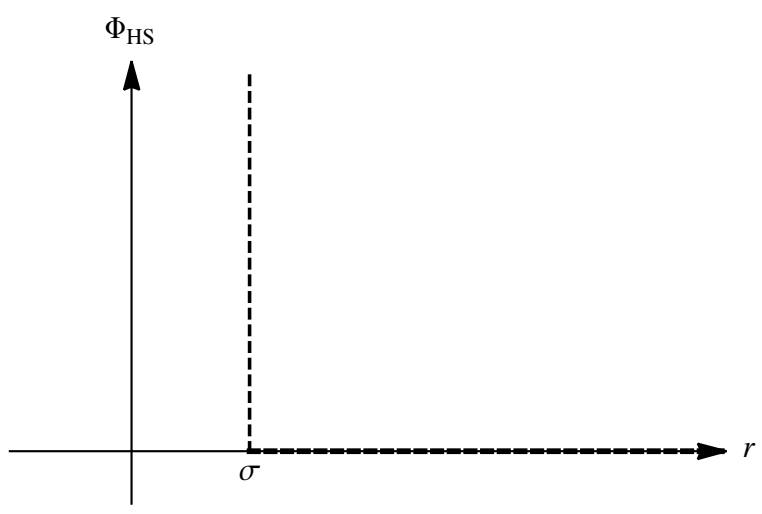

(a)

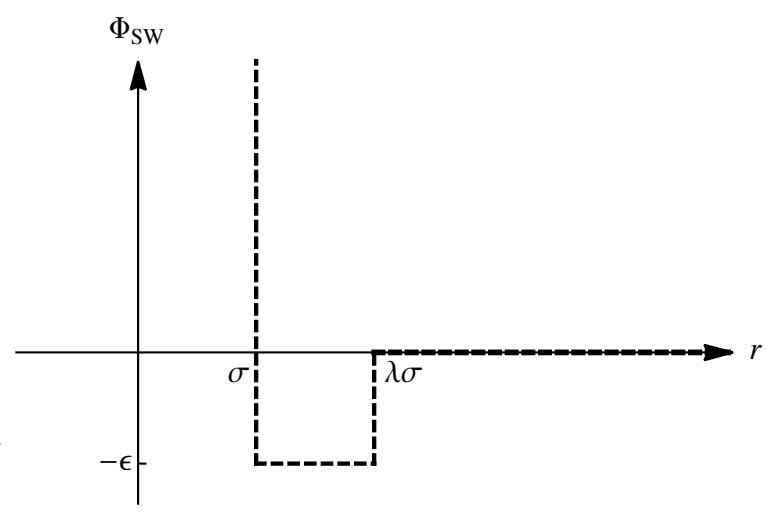

(b)

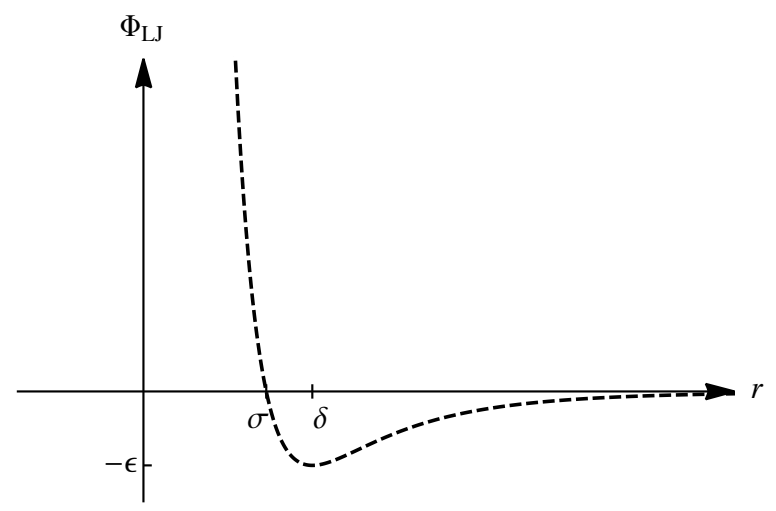

(c)

Figura 2.2: Potenciales intermoleculares binarios empíricos (línea a trazos) y su comportamiento con respecto a la distancia $r$. Potencial de esfera dura (a), de pozo cuadrado (b) y el potencial Lennard-Jones (c).

donde ambas distancias características están relacionadas mediante $\sigma=2^{-1 / 6} \delta$. La forma matemática de este potencial reproduce de forma básica el comportamiento real entre las moléculas: atracción entre ellas cuando están muy alejadas y repulsión si se encuentran muy cerca.

El primer término del lado derecho corresponde a la energía de corto alcance en su forma más simplificada dada por la ecuación 2.8 . Aunque no existe fundamento teórico para la elección de la potencia del término repulsivo, Lennard-Jones mostró de manera empírica [17] que valores entre 8 y 14 son adecuados para la construcción del segundo coeficiente virial en gases nobles; por conveniencia matemática se elige el valor $n=12$. El término repulsivo del lado derecho de (2.8) es precisamente el primer sumando de la ec. (2.9) correspondiente a la energía de dispersión 13

\section{Kihara}

El potencial de Kihara (K), se aplica en general a moléculas no esféricas que son sustituidas por cuerpos rígidos convexos (ver apéndice C.2 y rodeados de un campo energético de la misma forma [16]. Su expresión

${ }^{13} \mathrm{El}$ potencial Lennard-Jones es un caso especial del llamado potencial de Mie, cuya forma general está dada por

$$
\Phi_{\mathrm{Mie}}(r)=\frac{\nu}{r^{n}}-\frac{\mu}{r^{m}}, \quad n>m>3,
$$

con parámetros $\nu$ y $\mu$ constantes. 
analítica tiene la misma forma funcional a la del potencial LJ 12-6:

$$
\Phi_{\mathrm{K}}=\Phi_{\mathrm{LJ}}\left(\rho ; \rho_{0}, \epsilon\right)=\epsilon\left[\left(\frac{\rho_{0}}{\rho}\right)^{12}-2\left(\frac{\rho_{0}}{\rho}\right)^{6}\right]
$$

donde $\rho_{0}$ es la distancia mínima a la que pueden acercarse las superficies de dos moléculas sin sentir atracción entre ellas y $\rho$ es la distancia más corta entre sus superficies. Es decir, $\rho$ depende de la distancia $r$ entre los centros de masa de las moléculas, de su forma y de la configuración orientacional entre ellas.

\section{Stockmayer}

El potencial Stockmayer (SM) fue construido para estudiar las propiedades de sistemas polares y consiste en la interacción entre dos dipolos puntuales más una interacción tipo Lennard-Jones [10,11,13,18]. En el caso de un sistema conformado por moléculas de una sola especie (cada una con momento dipolar $\mu$ ) está dado por 14

$$
\Phi_{\mathrm{SM}}\left(r_{i j}, \boldsymbol{\omega}_{i}, \boldsymbol{\omega}_{j}\right)=\Phi_{\mathrm{LJ}}(r ; \epsilon, \delta)-\frac{\mu^{2}}{r^{3}} g_{i j}\left(\boldsymbol{\omega}_{i}, \boldsymbol{\omega}_{j}\right)
$$

donde $\boldsymbol{\omega}_{i}=\left(\theta_{i}, \phi_{i}\right)$ representa el conjunto de ángulos que definen la orientación del dipolo de la molécula $i$-ver figura 2.3 y $r$ es la distancia entre los dipolos. La función $g_{i j}$ que aparece en esta última ecuación está dada por

$$
\begin{aligned}
g_{i j}\left(\boldsymbol{\omega}_{i}, \boldsymbol{\omega}_{j}\right) & =\mathbf{s}_{i} \cdot \mathbf{s}_{j}-\frac{3}{r^{2}}\left(\mathbf{s}_{i} \cdot \mathbf{r}\right)\left(\mathbf{s}_{j} \cdot \mathbf{r}\right) \\
& =2 \cos \theta_{i} \cos \theta_{j}-\sin \theta_{i} \sin \theta_{j} \cos \left(\phi_{i}-\phi_{j}\right)
\end{aligned}
$$

donde $\mathbf{s}_{i}$ es el vector unitario de cada dipolo. Diversas modificaciones y extensiones a este potencial son utilizados regularmente en el estudio de sistema polares a nivel teórico [19] y a nivel computacional [20] y posteriormente serán revisadas con mayor detalle (pág. 46p.

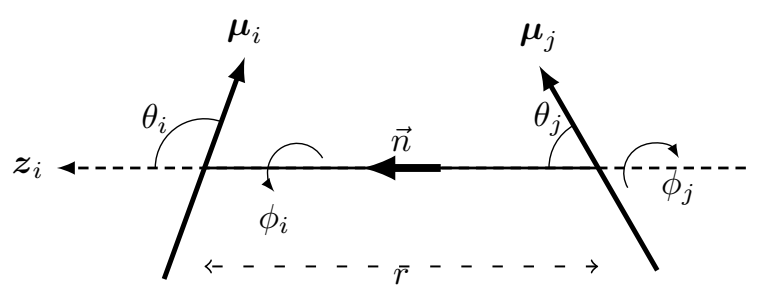

Figura 2.3: Configuración angular entre moléculas SM. Ángulos entre dos dipolos puntuales con intensidades $\boldsymbol{\mu}_{i}$ y $\boldsymbol{\mu}_{j}$ y separación $r$.

\subsection{Ecuación de estado del virial}

\subsubsection{Introducción}

De todas las EDE propuestas la más versátil para el estudio de gases es aquella obtenida como un desarrollo del virial en la presión $P$ (o el volumen $V$ ) el cual, para un sistema de un componente, está dado por [3,6,13]

$$
\frac{P}{\rho k_{\mathrm{B}} T}=1+\sum_{n=2} B_{n}(T) \rho^{n-1}
$$

\footnotetext{
${ }^{14}$ Este potencial y la teoría que lo rodea es revisado con mayor detalle al inicio del capítulo 4
} 
donde $\rho=N / V$ es la densidad. El término $B_{n}(T)$, con una dependencia explícita con la temperatura, es conocido como el $n$-ésimo coeficiente virial. Estos coeficientes indican la desviación existente en una EDE real de la de un gas ideal. La ventaja de usar esta EDE es que los coeficientes que la componen pueden escribirse en términos del tipo de interacción existente entre las moléculas y su cálculo requerirá del conocimiento analítico de dicha interacción la cual, en principio, caracteriza por completo la termodinámica del gas de estudio.

La expresión matemática de cada uno de los términos $B_{n}$ tiene un fundamento mecánico estadístico y la literatura sobre el tema es muy extensa en cuanto a su planteamiento básico $[6,21,22]$ y en el apéndice A.1 se hace un resumen sobre los principales aspectos al respecto. Debido a su importancia en el estudio de fluidos en fase gaseosa, a continuación se exponen los detalles de los dos primeros coeficientes viriales, $B_{2}$ y $B_{3}$.

\subsubsection{Primeros coeficientes viriales}

\section{Segundo coeficiente virial}

La primera desviación de la idealidad esta dada por el coeficiente $B_{2}$ el cual puede escribirse en términos de la segunda integral de racimo $b_{2}$, expresión definida en el apéndice A.1. Con esta relación, la evaluación de $B_{2}(T)$ requiere tanto la primera y segunda integrales de configuración, $Q_{1}^{C}=V$ y $Q_{2}^{C}$, ecuaciones A.11, por lo que el segundo coeficiente virial se reduce a la integral

$$
B_{2}=-\frac{1}{2 V} \int d \boldsymbol{r}_{1} d \Omega_{1} d \boldsymbol{r}_{2} d \Omega_{2}\left[\mathrm{e}^{-\Phi / k_{\mathrm{B}} T}-1\right],
$$

donde se ha incluido una dependencia angular en la función del potencial de interacción por pares $\Phi$.

Si el volumen $V$ es macroscópico, la ausencia de fuerzas externas significa que $\Phi$ depende únicamente de la posición relativa entre las moléculas $\boldsymbol{r}=\boldsymbol{r}_{1}-\boldsymbol{r}_{2}$ y la orientación. En este caso general introducimos nuevas coordenadas $\boldsymbol{r}$ y la posición $\boldsymbol{r}_{\mathrm{CM}}$ del centro de masa de dos moléculas para obtener

$$
B_{2}=-\frac{1}{2 V} \int d \boldsymbol{r}_{\mathrm{CM}} d \boldsymbol{r} d \Omega_{1} d \Omega_{2}\left[\mathrm{e}^{-\Phi / k_{\mathrm{B}} T}-1\right] .
$$

Debido a que el integrando no depende de la distancia entre los centros de las moléculas, la integral sobre $\boldsymbol{r}_{\mathrm{CM}}$ puede ser desarrollada inmediatamente obteniéndose un factor de $V$. La posición relativa $\boldsymbol{r}$ es determinada por la distancia relativa $r$ y los ángulos $(\theta, \varphi)$ que especifican su orientación en el espacio. Los otros ángulos necesarios para especificar la orientación intermolecular, asumiendo que la molécula 1 está fija en el espacio, son $\left(\theta_{2}, \varphi_{2}, \psi_{2}\right)$; si se designa $\Omega=\left(\theta_{1}, \varphi_{2}, \theta_{2}, \varphi_{2}, \psi_{2}\right)$ el coeficiente puede ser escrito com ${ }^{15}$

$$
B_{2}(T)=-\frac{1}{2 V} \int_{0}^{\infty} d r r^{2} \int d \Omega\left[\mathrm{e}^{-\Phi(r, \Omega) / k_{\mathrm{B}} T}-1\right] .
$$

Para moléculas esféricas $\mathrm{e}^{-\Phi(r, \Omega) / k_{\mathrm{B}} T}=\mathrm{e}^{-\Phi(r) / k_{\mathrm{B}} T}$, ésta ecuación simplemente da

$$
B_{2}(T)=-2 \pi \int_{0}^{\infty} d r r^{2}\left[\mathrm{e}^{-\Phi(r) / k_{\mathrm{B}} T}-1\right]
$$

donde la integración al infinito se permite mientras el potencial intermolecular cae a cero para valores de $r$ más grandes que unos pocos diámetros moleculares. En general, el segundo coeficiente virial puede calcularse numéricamente con un modelo conveniente para $\Phi(r)$. Alternativamente, la información sobre el potencial intermolecular puede desarrollarse a partir de medidas experimentales del segundo coeficiente virial.

Típicamente, como se observa en la figura 2.4, este coeficiente es negativo a temperaturas bajas mientras que a temperaturas mayores el coeficiente se hace positivo. Esto se debe a la competencia entre los términos energéticos negativos y positivos. A temperaturas mucho más altas el segundo coeficiente generalmente tiende a cero debido a que las interacciones comienzan a anularse [1]. El coeficiente $B_{2}$ es una función tan solo de

\footnotetext{
${ }^{15}$ Esto significa que el elemento del ángulo sólido correspondiente se toma como $\sin \theta d \theta d \varphi / 4 \pi$.
} 
la temperatura $T$ pero también dependerá de las variables introducidas como parámetros que especifiquen la función $\Phi(r, \Omega)$. Para garantizar la convergencia de la integral 2.20 en $r$ se supone que para cualquier $\Omega$ la cantidad $\int d \Omega \Phi(r, \Omega) \rightarrow 0$ al menos tan rápido como $1 / r^{4}$ cuando $r \rightarrow \infty$.

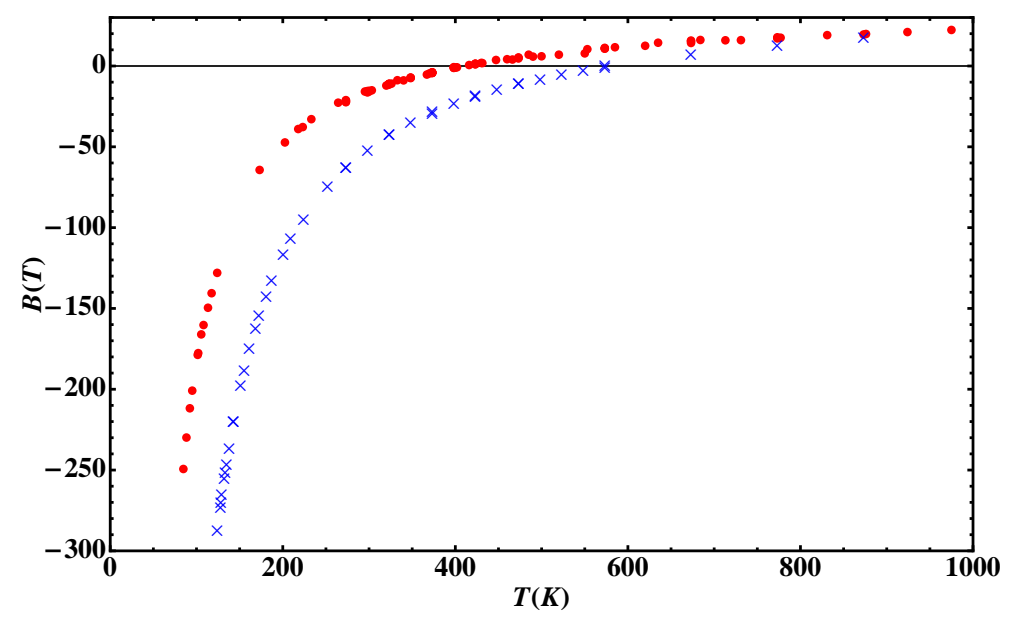

Figura 2.4: Segundo coeficiente virial experimental $B(T)$, para $\operatorname{Ar}(\bullet)$ y para el $\operatorname{Kr}(\times)$ y su dependencia con la temperatura [23].

\section{Tercer coeficiente virial}

En el caso del tercer coeficiente virial $B_{3}$ se considerarán (con el fin de simplificar los principales resultados) potenciales intermoleculares centrales que dependen tan solo de la distancia de separación $r_{i j}$ entre dos partículas cualesquiera; por lo tanto, la energía configuracional se puede aproximar mediante la aditividad por pares, ec. (2.6).

Con lo anterior la tercer integral de racimo definida en el apéndice A requiere la obtención de $Q_{1}^{C}, Q_{2}^{C}$ y $Q_{3}^{C}$ dadas por las ecuaciones A.11, lo que lleva a la expresión final

$$
B_{3}(T)=\frac{1}{3 V} \iiint_{V} f_{12} f_{13} f_{23} d \boldsymbol{r}_{1} d \boldsymbol{r}_{2} d \boldsymbol{r}_{3}
$$

donde el término $f_{i j}$ dado por

$$
f_{i j}=\mathrm{e}^{\Phi\left(r_{i j}\right) / k_{\mathrm{B}} T}-1
$$

conocido como función de Mayer, se incluye con la intención de simplificar la notación. La integración analítica de la ec 2.22 es mucho más difícil que la del segundo coeficiente virial y los resultados analíticos o numéricos obtenidos para diversos potenciales intermoleculares no siempre son satisfactorios con respecto a los datos experimentales. Una mejoría a lo anterior es alcanzada cuando se incluyen términos de no aditividad [8] aunque se requieren procedimientos numéricos más complejos.

El desarrollo del virial es la forma conveniente para la ecuación de estado de gases reales a densidades moderadas. Es válida para temperaturas por debajo de la crítica y hasta la línea de saturación, aunque para temperaturas supercríticas la región de convergencia no es completamente clara. Debido a que sólo el segundo y tercer coeficiente pueden determinarse experimental o teóricamente, la aplicación de la ecuación del virial se limita a densidades hasta la mitad, e incluso mayores, de la crítica [21,24].

La forma matemática de las interacciones intermoleculares presentadas en el apartado 2.2.3] permite resolver analíticamente las integrales de los primeros dos coeficientes viriales; en el apéndice se incluye una sección dedicada a exponer algunos de ellos y sus principales características, ver A.2. Las expresiones ahí mostradas, al 
igual que el respectivo desarrollo de integración, han sido ocupadas en el transcurso de este trabajo y por ello el interés de presentarlas. Para simplificar la notación usada hasta el momento se aplicará la simbología clásica de usar las primeras letras del alfabeto (mayúsculas) para distinguir los primeros coeficientes viriales; es decir, $B$ corresponderá al segundo coeficiente virial, $C$ al tercer coeficiente, y así sucesivamente.

\subsection{Principio de estados correspondientes}

\subsubsection{Introducción}

Entre las múltiples herramientas con que cuenta la física de fluidos existe una que ha mostrado un gran poder en la búsqueda de propiedades termodinámicas de fluidos simples y sus mezclas: el principio de estados correspondientes (PEC) [7,14,25]. La versatilidad de dicho principio radica en que permite predecir propiedades de fluidos con información mínima del sistema de interés partiendo de un estudio detallado de algún sistema de referencia. El principio se basa en el análisis dimensional de las propiedades de los sistemas involucrados ya sea desde una visión macroscópica o partiendo de un aspecto molecular.

Demostrado por primera vez por van der Waals, el PEC establece que existen grupos de sustancias para los cuales es posible encontrar una EDE única. Tal como fue deducida, la EDE de van der Waals de cada sustancia se reduce con sus respectivos parámetros críticos de tal forma que

$$
F\left(\frac{V}{V_{c}}, \frac{T}{T_{c}}, \frac{P}{P_{c}}\right)=0
$$

es una ecuación válida para un conjunto específico de sustancias.

\subsubsection{PEC para sistemas simples}

Aunque el PEC fue establecido inicialmente desde un punto de vista empírico, trabajos posteriores han sentado un fundamento teórico utilizando a los parámetros moleculares de un potencial de interacción como parámetros de reducción. Los puntos básicos que conducen a este principio para sistemas simples (PEC simple), a nivel molecular, son enunciados a continuación:

1. La función de partición puede factorizarse en una contribución independiente de la densidad y otra del tipo configuracional dependiente de ella. Este punto es válido generalmente a bajas densidades y aún a altas para fluidos simples aunque no siempre aplica a moléculas poliatómicas o asociadas.

2. La contribución configuracional puede tratarse usando mecánica estadística clásica excluyendo de esa forma a aquellos fluidos que exhiben un comportamiento cuántico.

3. La energía potencial intermolecular total $\Phi$ se expresa como la suma de las interacciones por pares de moléculas, ec. (2.6), que permite escribir al potencial en la forma,

$$
\Phi=\epsilon f\left(\frac{r}{\delta}\right),
$$

que es el producto entre un parámetro de energía y una función "universal" $f$ específica para un grupo de sustancias; esta función depende tan solo de la distancia entre las moléculas $r$ reducida con una longitud $\delta$ característica del potencial intermolecular. Aquellos sistemas que cumplen este último punto, ec. 2.25, se les conoce como fluidos conformales, donde cada uno de ellos tiene parámetros $\delta$ y $\epsilon$ característicos [7,21].

Con este último enunciado la integral de configuración A.8 puede escribirse comd

$$
Q_{N}^{C}=\delta^{3 N} f^{*}\left(T^{*}, V^{*}, N\right)
$$

\footnotetext{
${ }^{16}$ Para una revisión más detallada se pueden consultar las referencias [14, 25].
} 
donde $f^{*}$ es una función común a las sustancias del grupo estudiado y $T^{*}=k_{\mathrm{B}} T / \epsilon$ y $V^{*}=V / \delta^{3}$ son cantidades adimensionales denominadas temperatura y volumen reducidos, respectivamente. Esto permite a su vez, definir a la función de partición total, a partir de la cual [14] se obtiene una EDE única de la forma

$$
P^{*}=\frac{P \delta^{3}}{\epsilon}=F\left(T^{*}, V^{*}\right),
$$

donde $F$ es una función común al grupo de sustancias y con una dependencia en los parámetros del potencial intermolecular.

El primer punto es válido para bajas densidades y fluidos monoatómicos aún a altas densidades; no lo es para fluidos poliatómicos ó cuando hay presencia de enlaces de hidrógeno. El segundo punto se satisface excepto para moléculas muy ligeras como el $\mathrm{H}_{2}$, He y Ne. Aunque en el tercer punto se precisa la aditividad por pares, ésta en realidad no es obligatoria ya que el PEC simple es obedecido para algunas sustancias aún a alta densidades [26].

\subsubsection{Extensiones al PEC simple}

Distintas modificaciones han sido realizadas al PEC simple para poder aplicarse a sistemas más complejos y todas ellas llevan de manera implícita la ausencia de no-conformalidad entre esos sistemas. En este sentido se han desarrollado dos métodos: el primero toma a la propiedad de interés y la desarrolla en una serie de Taylor con respecto a un conjunto de parámetros adimensionales que la caracterizan [7]. Procediendo de esta manera con el factor de compresibilidad se tiene

$$
Z\left(V^{*}, T^{*} ; \lambda_{1}, \cdots, \lambda_{n}\right)=\left.Z\left(V^{*}, T^{*}\right)\right|_{\lambda_{i}=0}+\sum_{i=1}^{n}\left(\frac{\partial Z}{\partial \lambda_{i}}\right)_{\lambda_{i}=0} \lambda_{i}
$$

en donde $\left\{\lambda_{i}\right\}$ son parámetros adimensionales de caracterización.

El segundo enfoque extiende el PEC simple partiendo de una visión molecular del sistema analizado. Esto se logra haciendo que los parámetros para un modelo intermolecular $\Phi$, propuesto ad hoc al sistema estudiado, dependan del estado termodinámico. Es evidente que la manera en que se logra esta dependencia está ligada directamente a la forma analítica de $\Phi$ y a la fiabilidad que se intente alcanzar con el método. Aquel potencial dependiente del estado del sistema se le conoce regularmente como pseudopotencial o como potencial efectivo [9] ya que su forma analítica es más simple que la del potencial realista (u original) y reproduce de forma aproximada la propiedad termodinámica en estudio.

Un ejemplo ilustrativo aplicando esta visión es el realizado por Cook y Rowlinson [27] para sistemas gaseosos formados por moléculas elípticas y polares y cuya interacción depende fuertemente de la orientación entre ellas. En dicho estudio se valen de promedios en el ensamble canónico [11] con los cuales hallan potenciales efectivos sencillos que, además de depender del estado del gas, tienen una dependencia implícita con las características de la molécula tales como la forma o la intensidad del multipolo eléctrico que contiene. La determinación de un potencial efectivo y sus principales características se estudia con mayor detalle en la siguiente sección y es base importante del presente trabajo por lo que el concepto será utilizado continuamente de ahora en adelante.

\subsection{Potencial efectivo}

\subsubsection{Introducción}

Una adecuada elección para un modelo intermolecular (como los mencionados en la sección 2.2.3) al estudiar un fluido proviene muchas veces de un conocimiento previo sobre cómo interactúan las partículas que forman dicho sistema. Esto significa que al aplicar un modelo muy simple a un sistema complejo lleva, generalmente, a resultados inadecuados debido a que las distintas contribuciones energéticas existentes en el fluido real no han sido consideradas. Cuando el potencial intermolecular incorpora exitosamente todas esas contribuciones se le 
suele denominar "potencial realista", $\Phi_{r}$. El uso de un potencial realista único puede hacerse a un conjunto de sustancias afínes en términos de propiedades comúnes en ellas y la única diferencia entre un elemento y otro es la forma en que se parametriza al potencial realista. En general, ésto se hace con términos moleculares que caracterizan a una sustancia en específico; usualmente un término de energía y otro de distancia.

Modelos como el potencial Lennard-Jones 12-6 (ec. 2.12 representan adecuadamente a sistemas simples en los que las moléculas son prácticamente esféricas, donde los multipolos existentes son muy pequeños o no influye el efecto de la forma de la molécula; dichas características anisotrópicas entre las moléculas requieren modelos más elaborados. Este es el caso del potencial SM, ec. 2.16), que al sumar un término extra al potencial LJ agrega la dependencia orientacional y electrostática de largo alcance existente entre las moléculas polares. Estos dos potenciales son una muestra clara de modelos "realistas" que reproducen las interacciones en fluidos reales y ejemplifican claramente como $\Phi_{r}$ tiene que ser modificado para detectar efectos más estructurados entre las moléculas. Sin embargo, la generación de potenciales más complejos conlleva la aplicación de herramientas teóricas o computacionales también más complejas.

Distintos trabajos de investigación han superado la dificultad anterior utilizando una función que sustituya los aspectos básicos del potencial $\Phi_{r}$ dentro de un régimen característico de estados; claro está, dicha funcion debe superar en sencillez matemática a la original incorporando parámetros que correspondan con características moleculares de los constituyentes del sistema de estudio. A esta nueva función se le denominará "potencial efectivo" $\Phi_{\text {ef }}$ y su calidad como potencial sustituto de $\Phi_{r}$ (o incluso de $\Phi$ ) se basará en cuán bien reproduzcan las propiedades del sistema original 17

La forma analítica precisa de $\Phi_{\text {ef }}$ dependerá del sistema que se estudia, de la propiedad termodinámica a reproducir, del estado termodinámico en el que se plantea dicho estudio, así como de la exactitud que se requiere de dicho análisis. La identificación correcta de todas estas restricciones permitirá plantear una forma matemática conveniente para $\Phi_{\text {ef }}$. En una gran cantidad de casos una función esféricamente simétrica es suficiente para alcanzar dicha meta, en especial cuando se trabaja a densidades bajas, aún cuando las moléculas no sean totalmente esféricas ${ }^{18}$ Sin embargo, a medida que las sustancias de estudio se vuelven más complejas el modelo efectivo necesita replantearse ya sea incluyendo parámetros extras ó proponiendo una manera distinta de interactuar entre las moléculas.

Tomaremos el primer caso como una solución viable para los sistemas que serán estudiados; es decir, se supone que el potencial efectivo de dichos sistemas es esféricamente simétrico aún en el caso que exista una clara dependencia orientacional en la interacción original $\Phi$. Además, se supondrá, en la medida de lo posible, que el modelo efectivo sea una interacción por pares de tal manera que las contribuciones extras entre varios cuerpos: $\Phi_{2}, \Phi_{3}, \ldots, \Phi_{N}$ de la ecuación 2.5 sean incorporadas en la interacción efectiva por pares.

\subsubsection{Propiedad termodinámica efectiva}

Si consideramos que un potencial efectivo $\Phi_{\text {ef }}$, reproduce adecuadamente alguna propiedad termodinámica $X$ del sistema de interés en un estado $(\rho, T)$ determinado, entonces debe cumplirse

$$
X_{\mathrm{ef}}\left(\rho, T ; \Phi_{\mathrm{ef}}\right)=X(\rho, T ; \Phi(\Omega))
$$

donde el lado derecho corresponde al dato real de la propiedad estudiada y es escrito como un funcional del potencial real $\Phi \longdiv { 1 9 }$ El lado izquierdo pertenece a la propiedad termodinámica obtenida con el potencial efectivo

\footnotetext{
${ }^{17}$ La línea distintiva entre un potencial realista y uno efectivo suele ser muy ambigua y el uso de un término u otro dependerá totalmente del contexto en el que se esté trabajando.

${ }^{18} \mathrm{~A}$ bajas densidades el segundo coeficiente virial de moléculas no esféricas, como el $\mathrm{H}_{2} \mathrm{O}$ o el octano, es reproducido adecuadamente usando un potencial esférico. Incluso, para moléculas como el etano y el butano el tercer coeficiente virial también se obtiene con un potencial de este tipo.

${ }^{19} \mathrm{Al}$ igual que en el caso de $\Phi$, la misma definición es aplicable a un potencial realista $\Phi_{r}$ tal que
}

$$
X_{\mathrm{ef}}\left(\rho, T ; \Phi_{\mathrm{ef}}\right)=X\left(\rho, T ; \Phi_{r}\right),
$$


construido para dicho propósito. En el lado derecho de la ecuación se hace patente la dependencia de $\Phi$ con la orientación $\Omega$ entre las dos moléculas. La definición 2.29 lleva implícita la idea de reproducir un conjunto de estados termodinámicos por lo que $\Phi_{\text {ef }}$ tendrá una mayor valía mientras mayor sea este conjunto. La definición 2.29 para un potencial efectivo será aplicada continuamente cuando se traten propiedades termodinámicas efectivas de los sistemas involucrados.

Debido a su accesibilidad experimental y teórica la presión es una buena elección con la cual trabajar. Así, la última ecuación en términos de esta propiedad termodinámica se convierte en

$$
P_{\mathrm{ef}}\left(\rho, T ; \Phi_{\mathrm{ef}}\right)=P(\rho, T ; \Phi(\Omega)) .
$$

donde $\Omega$ es el conjunto de ángulos que definen la orientación entre dos moléculas que son supuestas rígidas. Si $g(r, \Omega ; \Phi(\Omega))$ es la función de distribución de dos partículas la ecuación anterior puede replantearse usando el teorema del virial de Clausius [10]

$$
\frac{P}{\rho k_{\mathrm{B}} T}=1-\frac{\rho}{6} \int d^{3} r \int d \Omega r \frac{\partial \Phi(\Omega)}{\partial r} g(r, \Omega ; \Phi(\Omega))
$$

donde $\Omega$ está normalizada, $\int d \Omega=1$. Al sustituir 2.32 en ambos lados de 2.31 y simplificando se obtiene

$$
\int_{0}^{\infty} d r r^{3} \frac{\partial \Phi_{\mathrm{ef}}}{\partial r} g_{\mathrm{ef}}\left(r ; \rho, T ; \Phi_{\mathrm{ef}}\right)=\int_{0}^{\infty} d r r^{3} \int d \Omega \frac{\partial \Phi}{\partial r} g(r ; \rho, T, \Omega ; \Phi(\Omega)) .
$$

Esta ecuación es una relación explícita y directa entre la función de distribución radial del sistema efectivo, $g_{\text {ef }}$ y la función de distribución radial originada por las distintas interacciones entre las partículas dadas en la ecuación 2.5. Es claro que ambos lados de la ecuación incluyen una dependencia en la densidad $\rho$ aunque, como se verá posteriormente, será preciso incluir dicha dependencia también en el potencial efectivo.

No existe un método único para encontrar $\Phi_{\text {ef }}$ a partir de cualquiera de las tres últimas ecuaciones y su búsqueda está sujeta a la información del sistema de estudio con que se cuente. Es decir, características básicas del sistema de interés como el estado termodinámico en que se trabaja, la información experimental, teórica, u obtenida a partir de simulación por computadora serán una fuente de información que permitirá realizar una elección matemática adecuada para $\Phi_{\text {ef }}$ y poder extraer así la información molecular requerida del sistema efectivo.

\subsubsection{Determinación de un potencial efectivo}

Si es que existe una solución $\Phi_{\text {ef }}$ para la ecuación (2.33), deberá estar acotada por un conjunto de funciones que garanticen la termodinámica "normal" del fluido y que satisfagan los siguientes requisitos físicos:

1. Tienen un mínimo único que separa una región repulsiva de una atractiva,

2. su parte repulsiva tiende a infinito cuando $r \rightarrow 0, \mathrm{y}$

3. su parte atractiva tiende a anularse cuando $r \rightarrow \infty$ más rápidamente que $1 / r^{3}$.

En general, aún suponiendo una forma funcional sencilla para este potencial surgen desventajas metodológicas en la resolución numérica de 2.33). Por ejemplo, si se conoce el lado derecho de la ecuación para un determinado número de estados y contando con las cantidades efectivas del lado izquierdo - obtenidas, ya sea por simulación o de forma teórica-, el potencial obtenido tendrá en general una dependencia con el estado termodinámico del sistema; es decir, un mismo potencial no funcionará para dos estados distintos. Aunado a esto, en caso de emplear un método iterativo, se requiere el manejo de una gran cantidad de información lo

lo que significa que el sistema efectivo se construye partiendo de las propiedades termodinámicas que genera un sistema realista elegido acorde al caso estudiado. 
que lleva a un ejercicio complejo e incierto. Lo anterior hace patente la necesidad de trabajar en un inicio con sistemas simples en los que los estados específicos estén a bajas densidades y/o altas temperaturas, y considerando moléculas cuasiesféricas no polares. En caso de alcanzar las expectativas con casos simples se está en posibilidad de estudiar sistemas de mayor complejidad.

\subsubsection{Características de un potencial efectivo}

El principal objetivo en la construcción de un potencial $\Phi_{\text {ef }}$ es que abarque una región amplia de estados $\rho-T$ con una forma matemática lo más sencilla posible, contando además con el menor número de parámetros efectivos. Como éstos pueden presentar una dependencia del estado termodinámico del sistema, una elección adecuada de la estructura matemática del potencial efectivo, tendrá una dependencia en dichos parámetros haciendo que el potencial cambie de manera suave en la región de estados en la que está definida. En principio, se esperaría que un potencial con dependencia del estado termodinámico sea capaz sólo de reproducir la propiedad termodinámica a partir de la cual se generó y dicha dependencia se mantendría aún suponiendo ausencia de interacciones de más de dos cuerpos; así, por ejemplo, una $\Phi_{\text {ef }}$ obtenida resolviendo la ecuación 2.31 solo tendría validez en la reproducción de la presión del sistema original [28]. Sin embargo, la experiencia ha mostrado que esto no siempre sucede y, en general, el mismo potencial efectivo (mismos parámetros efectivos) para una sustancia puede ser empleado con éxito en el cálculo de propiedades distintas de aquella con la que se obtuvo $\Phi_{\text {ef }}$.

Las formas más comúnes para un $\Phi_{\text {ef }}$ son las que están caracterizadas por dos parámetros y la inclusión de un parámetro extra permite, generalmente, tener un mejor control del potencial efectivo. Este nuevo parámetro debe estar ligado a una propiedad molecular tal como la excentricidad en el caso de moléculas convexas o el momento dipolar para el caso de moléculas polares. En ese sentido la teoría ANC, como veremos mas adelante, constituye un modelo efectivo cuyas características engloban los requisitos arriba mencionados al usar una familia de potenciales triparamétricos en los cuales el parámetro extra controla la pendiente del potencial intermolecular por pares.

\subsection{Simulación molecular}

El estudio de fluidos mediante simulación por computadora se ha convertido en una herramienta de uso común que complementa al experimento y a la teoría con información relevante y que en muchas ocasiones (cuando se trabajan sistemas muy complejos) resulta ser la única manera de obtenerla. La información que se extrae por este medio ayuda a entender las propiedades de un conjunto de partículas en términos de su estructura y las interacciones microscópicas que se les asignen a ellas. Es decir, propuesta la interacción entre las moléculas, se pueden predecir de manera "exacta" (ateniéndose a la limitación numérica y computacional) las propiedades del sistema simulado. Lo anterior significa que además de implementar algoritmos eficientes, en máquinas que procesen los cálculos de manera mucho más rápida, la elección del modelo de interacción entre las partículas resulta de gran importancia.

Existen dos grandes grupos de técnicas de simulación en la mecánica estadística, la dinámica molecular (DM) y el método de Monte-Carlo (MC), que muchas veces suelen combinarse para dar lugar a métodos híbridos. En el método MC se evalúan los promedios de la mecánica estadística mediante la generación aleatoria, pesada por el factor de Boltzmann, de una configuración del sistema; su principal característica radica en que está limitada al cálculo de propiedades estáticas.

El objetivo de la dinámica molecular es resolver las ecuaciones de movimiento para obtener las trayectorias moleculares (en el espacio fase) de cada una de las partículas que forman el sistema propuesto. A continuación se muestran los aspectos principales de este método tanto para interacciones de corto como de largo alcance, incluyendo además, anisotropía orientacional entre las partículas. Posteriormente se presentan de manera breve 
las herramientas con las que se lleva el control de la dinámica y con las cuales se logra simplificar el trabajo de computadora.

\subsubsection{Ecuaciones de movimiento}

Para un sistema compuesto de $N$ átomos cuyos centros de masa se ubican con las coordenadas $\mathbf{r}^{N}=$ $\left(\mathbf{r}_{1}, \mathbf{r}_{2}, \cdots, \mathbf{r}_{N}\right)$ y con momentos lineales $\mathbf{p}^{N}=\left(\mathbf{p}_{1}, \mathbf{p}_{2}, \cdots, \mathbf{p}_{N}\right)$, todos ellos interactuando mediante un potencial por pares $\Phi_{2}(\mathbf{r})$, donde $\mathbf{r}=\mathbf{r}_{j}-\mathbf{r}_{i}$. Las ecuaciones clásicas de movimiento para cada partícula están dadas por

$$
\dot{\mathbf{r}}_{i}=\frac{\mathbf{p}_{i}}{m_{i}} \quad \quad \text { y } \quad \dot{\mathbf{p}}_{i}=-\frac{\partial \Phi_{2}}{\partial r_{i}}=\mathbf{F}_{i}
$$

donde $\mathbf{F}_{i}$ es la fuerza total sobre la partícula $i$ debida a todas las demás y a posibles campos externos. Cuando el potencial intermolecular $\Phi_{2}$ incluye una dependencia orientacional aparecen torcas sobre cada una de las partículas. Esto significa que además de la fuerza entre partículas existe una torca sobre cada una de ellas debida a un campo externo $\mathbf{G}_{i}$, tal que

$$
\mathbf{N}_{i}=\dot{\mathbf{L}}_{i}=\mathbf{s}_{i} \times \mathbf{G}_{i}
$$

donde $\mathbf{N}_{i}$ y $\mathbf{L}_{i}$ son la torca total y el momento angular correspondientes a la partícula $i$, y $\mathbf{s}_{i}$ es el vector unitario que indica su "orientación". En particular, cuando la molécula a simular tiene simetría lineal, el momento angular esta dado por $\mathbf{L}_{i}=I \boldsymbol{\omega}_{i}$ donde $I$ es su momento de inercia -para más detalles ver la sección C.1

Existen muchos métodos para desarrollar una integración numérica paso a paso de las ecuaciones (2.34) y 2.35 y la experiencia ha colocado a la familia de algoritmos tipo Verlet como uno de los mejores [29,30]. De todos ellos, el "velocity Verlet", ha mostrado una buena eficiencia ${ }^{20}$ En el caso traslacional, está dado por

$$
\begin{aligned}
\mathbf{p}_{i}\left(t+\frac{1}{2} \Delta t\right) & =\mathbf{p}_{i}(t)+\frac{1}{2} \Delta t \mathbf{F}_{i}(t) \\
\mathbf{r}_{i}(t+\Delta t) & =\mathbf{r}_{i}(t)+\Delta t \frac{\mathbf{p}_{i}\left(t+\frac{1}{2} \Delta t\right)}{m_{i}} \\
\mathbf{p}_{i}(t+\Delta t) & =\mathbf{p}_{i}(t)+\frac{1}{2} \Delta t \mathbf{F}_{i}(t+\Delta t)
\end{aligned}
$$

donde $\Delta t$ es el paso temporal. El símil al caso traslacional se logra con las ecuaciones rotacionales mediante el mismo algoritmo

$$
\begin{aligned}
\mathbf{s}_{i}(t+\Delta t) & =\mathbf{s}_{i}(t)+\Delta t \dot{\mathbf{s}}_{i}(t)+\frac{1}{2}(\Delta t)^{2} \ddot{\mathbf{s}}_{i}(t) \\
\dot{\mathbf{s}}_{i}\left(t+\frac{1}{2} \Delta t\right) & =\dot{\mathbf{s}}_{i}(t)+\frac{1}{2} \Delta t \ddot{\mathbf{s}}_{i}(t) \\
\dot{\mathbf{s}}_{i}(t+\Delta t) & =\dot{\mathbf{s}}_{i}\left(t+\frac{1}{2} \Delta t\right) \ddot{\mathbf{s}}_{i}(t+\Delta t) .
\end{aligned}
$$

A partir de la ecuación (C.7) se calculan directamente las dos primeras ecuaciones de la parte rotacional; sin embargo, la última de estas tres ecuaciones no puede obtenerse debido a la dependencia de $\ddot{\mathbf{s}}_{i}(t+\Delta t)$ con $\dot{\mathrm{s}}_{i}(t+\Delta t)$ que aún no se calcula en las ecuaciones anteriores. Por ello se aplica la aproximación

$$
\dot{\mathbf{s}}_{i}(t+\Delta t)=\dot{\mathbf{s}}_{i}\left(t+\frac{1}{2} \Delta t\right)+\frac{1}{2} \Delta t \ddot{\mathbf{s}}_{i}(t)
$$

con la que se logra calcular la aceleración angular

$$
\ddot{\mathbf{s}}_{i}(t+\Delta t)=I^{-1} \dot{\mathbf{G}}_{i}(t+\Delta t)-\left\{I^{-1} \mathbf{s}_{i}(t+\Delta t) \cdot \mathbf{G}_{i}(t+\Delta t)+\left[\dot{\mathbf{s}}_{i}\left(t+\frac{1}{2} \Delta t\right)+\frac{1}{2} \Delta t \ddot{\mathbf{s}}_{i}(t)\right]^{2}\right\} \mathbf{s}_{i}(t)
$$

de manera eficiente [32]. En el caso de que existan interacciones de largo alcance entre las partículas a menudo se emplea el método de sumas de Ewald presentado en la siguiente sección.

\footnotetext{
${ }^{20}$ Las principales características del algoritmo de Verlet son: (a) es reversible en el tiempo, (b) es simpléctico, (c) es de bajo orden en el tiempo, de aquí que permita pasos temporales grandes, (d) requiere una sola evaluación de la fuerza, y (e) es fácil de programar [31].
} 


\subsubsection{Método sumas de Ewald}

\section{El método para cargas}

Un sistema de $N$ átomos (cada uno de los cuales lleva una carga $q_{j}$ ) tiene una energía de interacción total carga-carga $(q q)$

$$
\Phi_{q q}=\frac{1}{2} \sum_{n}^{\prime} \sum_{i=1}^{N} \sum_{j=1}^{N} \frac{q_{i} q_{j}}{\left|\mathbf{r}_{i j}+L \mathbf{n}\right|}
$$

donde $L$ es la longitud de la caja. La suma es sobre todos los vectores enteros $\mathbf{n}$, y el símbolo prima indica que los términos con $i=j$ se omiten cuando $\mathbf{n}=0$. Esta suma converge lentamente debido al largo alcance de las fuerzas electrostáticas y de hecho es condicionalmente convergente.

El método de la suma de Ewald [29,30] transforma la suma 2.43 en dos sumas que convergen rápidamente más un término constante. Para ello se supone que cada partícula $i$ con carga $q_{i}$ es rodeada por una distribución de carga difusa de signo opuesto, tal que la carga total de esta nube cancela exactamente a $q_{i}$. En ese caso, el potencial electrostático debido a la partícula $i$ es debido exclusivamente a la fracción de $q_{i}$ que no es apantallada y a grandes distancias, esta fracción decae rápidamente a cero y dependerá de la distribución de carga apantallante.

La distribución de carga compensatoria que rodea a la carga $i$ es una gaussiana con anchura $\sqrt{2 / \alpha}$

$$
\rho_{i}(r)=-q_{i}\left(\frac{\alpha}{\pi}\right)^{3 / 2} \exp \left[-\alpha r^{2}\right]
$$

donde $\alpha$ es un parámetro arbitrario que determina la anchura de la distribución y su elección está determinada por consideración de eficiencia computacional.

La contribución al potencial electrostático en un punto $r_{i}$ debido a un conjunto de cargas apantalladas puede calcularse directamente por una suma, ya que el potencial debido a una carga apantallada es una función de $r$ que decae rápidamente. Finalmente, debe corregirse el hecho de haber sumado una nube apantallante a cada partícula, esto se muestra esquemáticamente en la figura 2.5 .
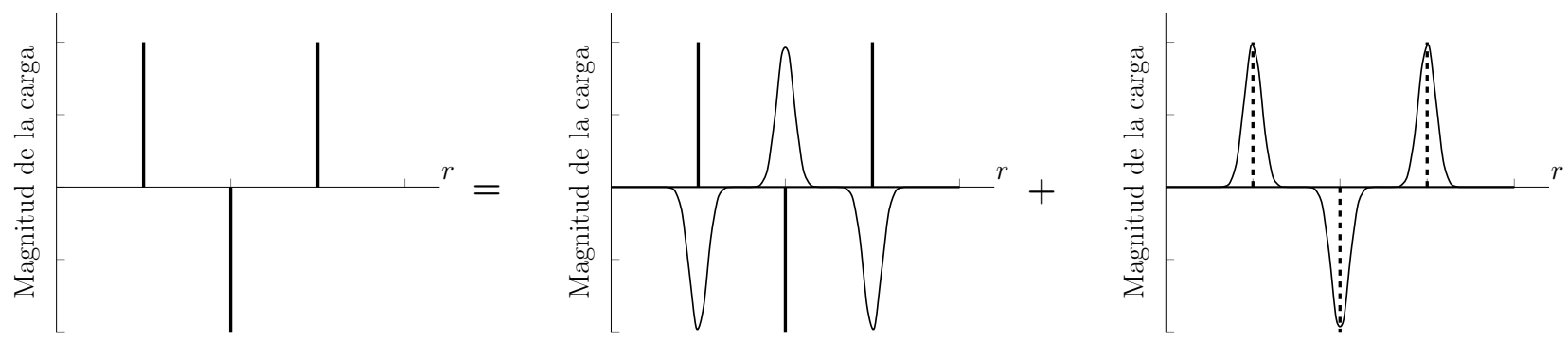

Figura 2.5: Componentes de una suma de Ewald en un sistema unidimensional de cargas puntuales. En el espacio directo, cada carga es rodeada por una distribución de carga gaussiana $\rho_{i}(\boldsymbol{r})$ de igual amplitud, pero de signo opuesto. Esta contribución es contrabalanceada en el espacio recíproco por una distribucuón gaussiana del signo contrario.

Así, se tienen tres contribuciones al potencial electrostático: una debida a la carga puntual, otra debida a la nube electrónica apantallante y finalmente otra debida a la nube electrónica compensatoria; las tres con carga $q_{i}$. Aunque debieran excluirse autointeracciones coulómbicas, es conveniente retener la contribución debida a la distribución de carga compensatoria y corregir posteriormente. La razón de esto último es que esta distribución no es solo una función que varía suavemente sino además es periódica. Tal función puede representarse por una serie de Fourier (rápidamente convergente), lo cual será esencial desde el punto de vista computacional.

La suma de Ewald consiste en reorganizar la ecuación 2.43 en sumas sobre cascarones esféricos concéntricos, considerando la neutralidad de carga $\sum_{j} q_{j}=0$. La suma incluye el parámetro $\alpha$ cuyo valor determina la 
máxima exactitud numérica y una elección conveniente de ellas asegura que términos del orden de $\exp \left(-\alpha^{2} L^{2}\right)$ sean despreciables por lo que se tiene que

$$
\begin{aligned}
\Phi_{q q}=\frac{1}{2 \pi L} \sum_{\mathbf{n} \neq 0} \frac{1}{n^{2}} \exp \left[-\frac{\pi^{2}|\mathbf{n}|^{2}}{L^{2} \alpha^{2}}\right] & \left|\sum_{j=1}^{N_{a}} q_{j} \exp \left(\frac{2 \pi i}{L} \mathbf{n} \cdot \mathbf{r}_{j}\right)\right|^{2} \\
& \sum_{1 \leq i \leq j \leq N_{a}} \frac{q_{i} q_{j} \operatorname{erfc}\left(\alpha\left|\mathbf{r}_{i j}\right|\right)}{\left|\mathbf{r}_{i j}\right|}-\frac{\alpha}{\pi^{1 / 2}} \sum_{j=1}^{N_{a}} q_{j}^{2} .
\end{aligned}
$$

Los términos reales en (2.45) son de corto alcance, así que un corte esférico (con alcance $r_{c u t}<L / 2$ ) puede usarse junto con las fronteras periódicas. La suma en el espacio de Fourier (sobre n) también será candidata a truncarse después de un número limitado de términos. Un valor típico para $\alpha$ es de $5 / L$, aunque el resultado será relativamente insensible a la elección, dado que existen términos suficientes en la $\mathbf{n}$-suma. Un corte esférico es impuesto sobre la $\mathbf{n}$-suma, $|\mathbf{n}|^{2} \leq n_{\text {cut }}^{2}$; típicamente $n_{\text {cut }}$ es cercano a 5 [33].

\section{El método para dipolos puntuales}

Para el caso dipolar el campo $\mathbf{G}_{i}$ que aparece en la ecuación 2.35 está dado por

$$
\mathbf{G}_{i}=\mu_{i} \mathbf{E}\left(\mathbf{r}_{i}\right)=-\nabla_{\mathbf{s}_{i}} \Phi_{d d}
$$

donde $\mathbf{s}_{i}$ es el vector direccional unitario del $i$-ésimo dipolo puntual, $\mu_{i}$. Si se considera que todos los dipolos tienen la misma intensidad $(\mu)$, la interacción dipolo-dipolo $(d d)$ puede ser escrita en la forma [33]

$$
\Phi_{d d}=-\mu^{2} \frac{\left(\mathbf{s}_{i} \cdot \nabla\right)\left(\mathbf{s}_{j} \cdot \nabla\right)}{\left|\mathbf{r}_{i j}\right|}
$$

donde es el término $\left(\mathbf{s}_{i} \cdot \nabla\right)$ puede tomarse como una carga $q$.

Si se sigue el mismo procedimiento como el que se aplica a las cargas el operador diferencial anterior puede aplicarse a 2.45) para obtener la energía potencial total de un sistema dipolar:

$$
\begin{aligned}
\Phi_{d d}=\mu^{2} \quad \sum_{1 \leq i \leq j \leq N_{a}} & {\left[a_{1}\left(r_{i j}\right)\left(\mathbf{s}_{i} \cdot \mathbf{s}_{j}\right)-a_{2}\left(r_{i j}\right)\left(\mathbf{s}_{i} \cdot \mathbf{r}_{i j}\right)\left(\mathbf{s}_{j} \cdot \mathbf{r}_{i j}\right)\right] } \\
& +\frac{2 \pi \mu^{2}}{L^{3}} \sum_{\mathbf{n} \neq 0} e(n)\left[C(\mathbf{n})^{2}+S(\mathbf{n})^{2}\right]-\frac{2 \alpha^{3} \mu^{2} N_{a}}{3 \pi^{1 / 2}}
\end{aligned}
$$

donde

$$
\begin{gathered}
a_{n}(r)=\left\{\begin{array}{cc}
\frac{\operatorname{erfc}(\alpha r)}{r^{3}}+2 \alpha \frac{\exp \left(-\alpha^{2} r^{2}\right)}{\pi^{1 / 2} r^{2}} & n=1 \\
-r^{-1} \frac{d a_{n-1}}{d r} & n=2,3
\end{array}\right. \\
\begin{array}{c}
e(n)=\frac{1}{n^{2}} \frac{\exp \left(-\pi^{2} n^{2}\right)}{L^{2} \alpha^{2}} \\
\left\{\begin{array}{l}
C \\
S
\end{array}\right\}(\mathbf{n})=\sum_{j=1}^{N}\left(\mathbf{n} \cdot \mathbf{s}_{j}\right)\left\{\begin{array}{c}
\cos \\
\sin
\end{array}\right\}\left(2 \pi \frac{\mathbf{n} \cdot \mathbf{r}_{j}}{L}\right)
\end{array}
\end{gathered}
$$

donde $\operatorname{erfc}(x)$ es la función error complementaria [34]. En el límite $\alpha \rightarrow 0$, la ecuación (2.48) se reduce al resultado del potencial dado por el dipolo sencillo, ec. 2.16,

$$
\Phi_{d d}=\mu^{2} \sum_{i<j} \frac{g_{i j}}{r_{i j}^{3}}
$$

donde $g_{i j}$ esta dada por la ec. 2.18. 


\section{Inclusión del método en las ecuaciones de movimiento}

Cuando se trabaja con un potencial cuyas contribuciones son interacciones esféricas de corto alcance (LJ, ANC, etc) más una de largo alcance como la $\Phi_{d d}$, las ecuaciones de movimiento deben involucrar la fuerza generada por el potencial dipolo-dipolo la cual consiste en una suma sobre todos los átomos truncada a una distancia de corte $r_{c u t}$ y otra suma en el espacio de Fourier truncada en $n_{c u t}$. Así, la contribución dipolar a la fuerza de un átomo simple está dada por

$$
\begin{array}{r}
\mathbf{F}_{i j}^{d d}=\mu^{2} \sum_{j(\neq i)}\left[\left(a_{2}\left(r_{i j}\right)\left(\mathbf{s}_{i} \cdot \mathbf{s}_{j}\right)-a_{3}\left(r_{i j}\right)\left(\mathbf{s}_{i} \cdot \mathbf{r}_{i j}\right)\left(\mathbf{s}_{j} \cdot \mathbf{r}_{i j}\right)\right) r_{i j}+a_{2}\left(r_{i j}\right)\left(\left(\mathbf{s}_{i} \cdot \mathbf{r}_{j}\right) \mathbf{s}_{i}+\left(\mathbf{s}_{i} \cdot \mathbf{r}_{j}\right) \mathbf{s}_{j}\right)\right]+ \\
\frac{8 \pi^{2} \mu^{2}}{L^{4}} \sum_{n \neq 0} e(n)\left(\mathbf{n} \cdot \mathbf{s}_{i}\right) \mathbf{n}\left[C(\mathbf{n}) \operatorname{sen}\left(\frac{2 \pi}{L} \mathbf{n} \cdot \mathbf{r}_{i}\right)-S(\mathbf{n}) \cos \left(\frac{2 \pi}{L} \mathbf{n} \cdot \mathbf{r}_{i}\right)\right]
\end{array}
$$

donde se ha utilizado el resultado $\nabla(\mathbf{s} \cdot \mathbf{r})=\mathbf{s}$ y la derivada de erfc $(\alpha x)$ [34].

La contribución del dipolo en la resolución de la ecuación de movimiento del vector dipolar $\mathbf{s}_{i}$ se presenta al final de la sección 2.6.1. El cálculo de $\mathbf{G}_{i}$, ec. 2.46, también puede expresarse como la suma de dos términos:

$$
\begin{aligned}
\mathbf{G}_{i}= & \mu^{2} \sum_{j(\neq i)}\left[-a_{1}\left(r_{i j}\right) \mathbf{s}_{j}+a_{2}\left(r_{i j}\right)\left(\mathbf{s}_{j} \cdot \mathbf{r}_{i j}\right) r_{i j}\right]- \\
& \frac{4 \pi^{2} \mu^{2}}{L^{3}} \sum_{n \neq 0} e(n) \mathbf{n}\left[C(\mathbf{n}) \cos \left(\frac{2 \pi}{L} \mathbf{n} \cdot \mathbf{r}_{i}\right)-S(\mathbf{n}) \operatorname{sen}\left(\frac{2 \pi}{L} \mathbf{n} \cdot \mathbf{r}_{i}\right)\right]
\end{aligned}
$$

\subsubsection{Control de la Dinámica Molecular}

\section{Condiciones periódicas de frontera y radio de corte}

A menos que los efectos de superficie sean de interés particular se puede trabajar con condiciones periódicas de frontera. Dicha solución computacional consiste en rodear la "caja de simulación" original con réplicas de sí misma. Además se adopta la convención de mínima imagen en la que cada uno de los átomos interactúa con los átomos, o sus imágenes, más cercanos en el arreglo periódico. En el transcurso de la dinámica, si un átomo deja la caja de simulación, la atención debe concentrarse en la imagen entrante - figura 2.6. Una consideración especial debe tomarse cuando las interacciones entre las moléculas no sean de corto alcance, como en el caso de sistemas cargados y dipolares - ver sección 2.6.2 -.

La obtención de las fuerzas interatómicas en una simulación de DM involucra, en principio, un gran número de cálculos entre pares; esto se hace considerando cada uno de los átomos $i$ y su interacción sobre todos los otros $j$ para calcular las separaciones $r_{i j}$. En la práctica, se acostumbra establecer un radio de corte $r_{c u t}$ y despreciar las interacciones entre átomos separados una distancia mayor a este radio; así, se tiene que $\Phi\left(r_{i j}\right)=0$ cuando $r_{i j}>r_{c u t}$, por lo que la simulación se saltará los cálculos de las fuerzas, evitando cálculos costosos, y considera al siguiente candidato $j$, — ver figura 2.6 .

\section{Lista de vecinos}

Cuando se usa el radio de corte, el tiempo para examinar todas las separaciones entre pares es proporcional al número $\frac{1}{2} N(N-1)$ de distintos pares en un sistema de $N$ átomos. Para ahorrar tiempo de cómputo se hace uso de una lista de átomos vecinos. La esfera de radio $r_{c u t}$, alrededor de un átomo particular, está rodeada por otra esfera más grande de radio $r_{\text {list }}$ como se muestra en la figura 2.7. En el primer paso de la simulación se construye una lista de todos los vecinos de cada uno de los átomos y sólo aquellos que aparecen en dicha lista son checados en la rutina del cálculo de fuerza. Esta lista se reconstruye continuamenmte y es importante hacer esto antes de que algunas partículas no enlistadas crucen la zona de "seguridad" y entren a la zona de interacción. 


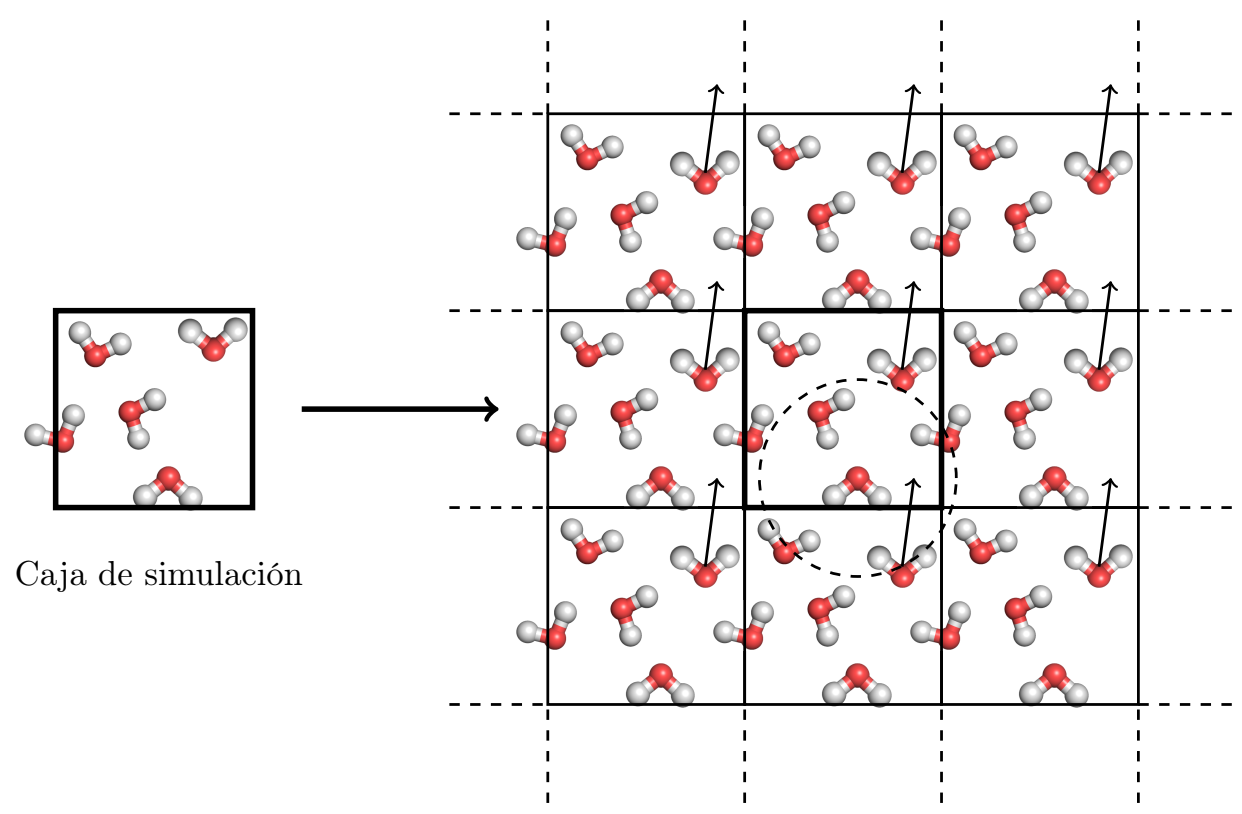

Figura 2.6: Condiciones periódicas de frontera. Mientras una partícula sale de la caja de simulación, una partícula imagen entra para reemplazarla. En el cálculo de las interacciones entre partículas dentro del radio de corte (círculo a trazos en la parte central de la figura) tanto los vecinos reales e imágenes son incluidos.

\section{Inicio de la simulación}

Establecida la caja de simulación de volumen fijo $V$, el inicio de la DM requiere el conjunto de posiciones y velocidades iniciales de las partículas. Evidentemente los resultados a obtener en la simulación deben ser independientes del estado del cual se parte por lo que cualquier configuración inicial es permitida mientras sea adecuada y con la densidad requerida. Cuando se parte de cero se acostumbra que las posiciones sean usualmente definidas sobre una red, suponiendo cierta estructura cristalina; esta estructura es típicamente la más estable a $T=0$ con el potencial respectivo.

Las velocidades iniciales para cada una de las partículas pueden definirse mediante el uso de una distribución de velocidades de Maxwell-Boltzmann a una temperatura fija [35]; éstas deben ser ajustadas ya que el sistema obtiene un pequeño momento lineal total correspondiente a un movimiento traslacional del sistema completo y por lo tanto es práctica común restar dicha componente de la velocidad para cada partícula y operar así en una condición de momento lineal total nulo.

Esta obtención aleatoria de las velocidades iniciales es usualmente el único momento donde el "azar" entra en una simulación de dinámica molecular; la subsecuente evolución temporal es completamente determinista. Una posibilidad alterna para inicializar las primeras posiciones y velocidades consiste en tomar los datos de posiciones y velocidades finales de una DM previa; ésta es de hecho la más comúnmente usada.

\section{Control del sistema}

En un cálculo estándar, la densidad es controlada mediante la elección del volumen de la caja y los cambios de la caja son usualmente alcanzados estableciendo un mecanismo en el código el cual lleva al sistema a la temperatura deseada reescalando las velocidades. En el algoritmo velocity Verlet esto puede implementarse reemplazando la ec. 2.37) por

$$
\mathbf{p}_{i}\left(t+\frac{\delta t}{2}\right)=\sqrt{\frac{T_{0}}{T(t)}} \mathbf{p}_{i}(t)+\frac{1}{2} \mathbf{F}_{i}(t) \delta t
$$




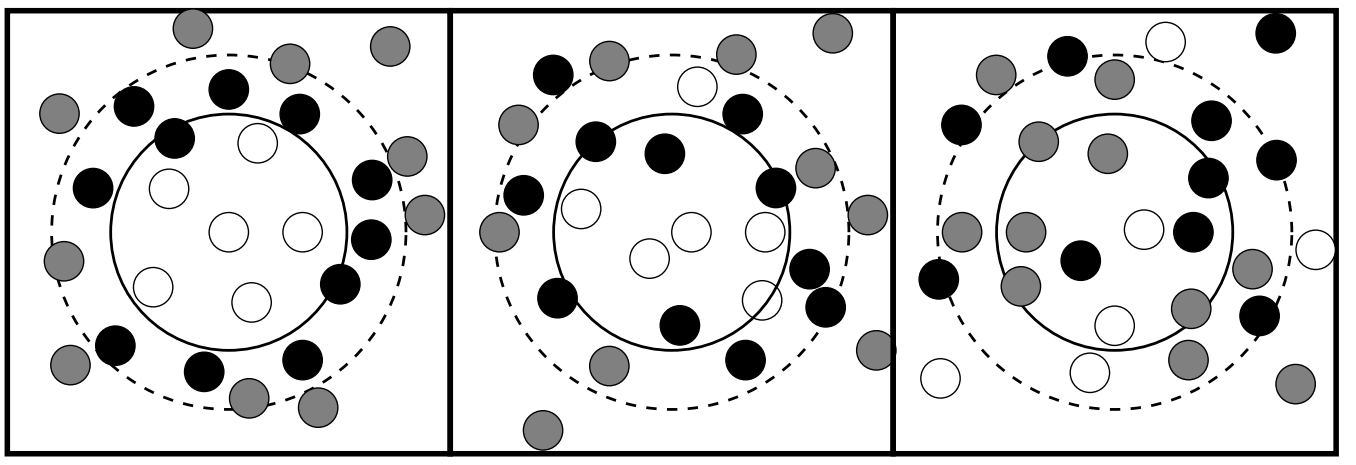

Figura 2.7: Lista de vecinos al momento de construirse y dos tiempos posteriores. Las esferas con radios de corte, $r_{\text {cut }}$ (círculo relleno), y para la lista de vecinos, $r_{l i s t}$ (círculo a trazos), son indicados en la figura. La lista debe reemplazarse antes de que las partículas (aquellos que aparecen en gris), originalmente fuera de la esfera exterior, penetren la esfera interna.

donde $T_{0}$ es la temperatura deseada, y $T(t)$ la "temperatura instantánea" - ver sección 2.6.4. Al lograr tal modificación no se están siguiendo las ecuaciones de Newton y la energía total no se conserva. Por lo tanto, no deben recolectarse datos en esta etapa: estas simulaciones de "temperatura controlada" deben usarse solamente para llevar al sistema de un estado a otro [36].

\subsubsection{Cantidades estadísticas a medir}

Las propiedades más fáciles de obtener en un sistema cuando alcanza el equilibrio son aquellas introducidas en termodinámica elemental tales como la energía o la presión y pueden ser expresadas en términos del estado del sistema. Su obtención es relativamente simple y permite vincular la termodinámica con el mundo microscópico.

\section{Propiedad instantánea}

La medición de cantidades en DM significa desarrollar promedios temporales de las propiedades físicas instantáneas del sistema y pueden ser escritas en función de las coordenadas y velocidades de la partícula. Así, se puede definir el valor instantáneo de una propiedad física genérica $A$ al tiempo $t$ :

$$
A(t)=f\left(\mathbf{r}_{1}(t), \cdots, \mathbf{r}_{N}(t), \mathbf{v}_{1}(t), \cdots, \mathbf{v}_{N}(t)\right)
$$

y obtener entonces su promedio

$$
\langle A\rangle=\frac{1}{N} \sum_{t=1}^{N} A(t)
$$

donde $N$ es el número de partículas. El valor de $A(t)$ se calcula cada paso temporal por la DM mientras ella se ejecuta y la suma 2.57) se actualiza a cada paso y al final de la ejecución se promedia con el número de pasos. Este es el método preferido cuando la cantidad es simple de calcular y/o particularmente importante.

\section{Energía cinética y potencial}

Las energías potencial y cinética promedio (instantáneas) para un sistema simple son obtenidas de manera inmediata durante el cálculo de la fuerza y las velocidades con las expresiones

$$
\begin{aligned}
V_{\text {trans }}(t) & =\sum_{i} \sum_{j>i} \Phi\left(\left|\mathbf{r}_{i}(t)-\mathbf{r}_{j}(t)\right|\right) \\
K_{\text {trans }}(t) & =\frac{1}{2} \sum_{i} m_{i}\left[v_{i}(t)\right]^{2}
\end{aligned}
$$


En el caso de que las moléculas puedan rotar tanto la energía cinética como la potencial del sistema se obtienen considerando las dos contribuciones del movimiento traslacional y rotacional. La energía potencial completa se obtiene sumando todas las contribuciones por pares del nuevo potencial de la misma forma que 2.58 y en el caso de la cinética se tiene que

$$
K=K_{\text {trans }}+K_{\text {rot }}
$$

donde $K_{\text {trans }}$ está dada por la ecuación 2.59 y la contribución rotacional, para el caso de sistemas dipolares, por

$$
K_{\text {rot }}=\frac{1}{2} I \sum_{i=1}^{N}\left|\dot{\mathbf{s}}_{i}\right|^{2}=\frac{1}{2} I \sum_{i=1}^{N}\left(\dot{\theta}_{i}^{2}+\dot{\varphi}_{i}^{2} \operatorname{sen}^{2} \theta_{i}\right)
$$

La suma de las energías cinética y potencial totales debe mantenerse constante durante toda la simulación por lo que se debe calcular en todo momento para asegurar constancia. En la práctica, existen pequeñas fluctuaciones en la energía total, las cuales son producidas usualmente por error en el tiempo de integración, y pueden reducirse en magnitud disminuyendo el valor del paso temporal.

\section{Temperatura}

La temperatura está directamente relacionada a la energía cinética por el teorema de equipartición asignando una energía cinética promedio $k_{\mathrm{B}} T / 2$ por grado de libertad. Definida la energía cinética instantánea en automático se puede definir la temperatura instantánea proporcional a $K(t)$

$$
T(t)=\frac{2}{m N k_{\mathrm{B}}} K(t)
$$

donde $m=3$ se utiliza para sistemas esféricos y $m=5$ cuando se trabaja con sistemas con simetría lineal como en el caso de los dipolos. La existencia de restricciones globales en el sistema reduce el número de grados de libertad por lo que deben ser restadas de la ecuación de arriba.

Separando las contribuciones energéticas por medio del teorema de equipartición también se pueden definir temperaturas correspondientes a la parte translacional y rotacional dadas por

$$
\begin{aligned}
T_{\text {rot }}(t) & =\frac{1}{N k_{\mathrm{B}}} K_{\text {rot }}(t) \\
T_{\text {trans }}(t) & =\frac{2}{3 N k_{\mathrm{B}}} K_{\text {trans }}(t)
\end{aligned}
$$

\section{Presión}

La medida de la presión en una simulación de dinámica molecular se basa en el teorema del virial de Clausius

$$
P V=N k_{\mathrm{B}} T-\frac{1}{3}\left\langle\mathbf{r}_{i} \cdot \mathbf{F}_{i}^{t o t}\right\rangle
$$

donde la fuerza completa sobre cada partícula tiene contribuciones del potencial esférico $\mathbf{F}_{i}^{s p h}$ y del anisotrópico $\mathbf{F}_{i}^{d d} ;$ es decir

$$
\left\langle\mathbf{r}_{i} \cdot \mathbf{F}_{i}^{t o t}\right\rangle=\left\langle\sum_{i}^{N} \mathbf{r}_{i} \cdot \mathbf{F}_{i}^{s p h}\right\rangle+\left\langle\sum_{i}^{N} \mathbf{r}_{i} \cdot \mathbf{F}_{i}^{d d}\right\rangle
$$

todas las cantidades que aquí aparecen son accesibles en una simulación por lo que la ecuación (2.64) constituye una forma práctica de medir la presión. 


\section{Estructura - Función de distribución radial}

Existen correlaciones bien definidas que pueden medirse experimentalmente para obtener detalles importantes sobre la organización molecular promedio. El tratamiento de la correlación estructural comienza con una función de distribución de pares completamente general:

$$
g\left(\mathbf{r}_{1}, \mathbf{r}_{2}\right)=\frac{N(N-1) \int d \mathbf{r}_{3} \cdots d \mathbf{r}_{N} \exp \left[-\frac{\Phi}{k_{\mathrm{B}} T}\right]}{\rho^{2} \int d \mathbf{r}_{1} \cdots d \mathbf{r}_{N} \exp \left[-\frac{\Phi}{k_{\mathrm{B}} T}\right]}
$$

donde la integral que aparece en el denominador es la función de partición. En el caso de sistemas homogéneos la separación relativa es significativa por lo que se llega a una suma sobre pares de átomos

$$
g(\mathbf{r})=\frac{2 V}{N}\left\langle\sum_{i<j} \delta\left(\mathbf{r}-\mathbf{r}_{i j}\right)\right\rangle
$$

y si además el sistema es isótropo la función puede se promediada sobre los ángulos sin pérdida de información. El resultado es la función de distribución radial $g(r)$, que describe la organización local esféricamente promediada alrededor de un átomo dado.

La definición de $g(r)$ implica que $\rho g(r) d \mathbf{r}$ sea proporcional a la probabilidad de encontrar un átomo en el elemento de volumen $d \mathbf{r}$ a una distancia $r$ de un átomo dado y $4 \pi \rho g(r) r^{2} \Delta r$ es el número promedio de átomos en una concha de radio $r$ y espesor $\Delta r$ centrada en el átomo.

La función de distribución radial se puede obtener en la simulación usando un histograma de separación discretizada entre pares de partículas. Si $h_{n}$ es el número de pares de átomo $(i, j)$ para los cuales $(n-1) \Delta \leq$ $r_{i j} \leq n \Delta r$. Si se supone que $\Delta r$ sea lo suficientemente pequeño se tiene que

$$
g\left(r_{n}\right)=\frac{V h_{n}}{2 \pi N^{2} r_{n}^{2} \Delta r}
$$

donde $r_{n}=(n-1 / 2) \Delta r$. Si la medición de la función de distribución radial se extiende a un rango máximo de $r_{d}$, el número de "rebanadas" del histograma será $r_{d} / \Delta r$.

\section{Referencias}

[1] L. E. Reichl. A modern course in statistical physics. John Wiley \& Sons, Inc., 1998.

[2] V. M. Faires and C. M. Simmang. Thermodynamics. MacMillan Publishing Co. Inc., 1978.

[3] H. B. Callen. Thermodynamics and an Introduction to Thermostatics. John Wiley and sons, 1985.

[4] F. Reif. Fundamentals of statistical and thermal physics. Mc Graw Hill Book Company, 1965.

[5] L. D. Landau and E. M. Lifshitz. Course of Theoretical Physics Vol 5, Statistical Physics Part 1. Pergamon International Library, 1980.

[6] R. K. Pathria. Statistical Mechanics. BH, Oxford, 2001.

[7] J. V. Sengers, R. F. Kayser, C. J. Peters, and H. J. Jr White. Equations of state for fluids and fluid mixtures. Part 1. IUPAC-Elsevier, 2000.

[8] I. G. Kaplan. Intermolecular Interactions: Physical Picture, Computational Methods and Model Potentials. John Wiley and Sons,Ltd, 2006.

[9] C. G. Gray and K. E. Gubbins. Theory of Molecular Fluids Volume 1: Fundamentals. Clarendon Press, Oxford, 1984. 
[10] G. C. Maitland, M. Rigby, E. B. Smith, and W. A. Wakeham. Statistical Intermolecular Forces. Clarendon Press, Oxford, 1981.

[11] J. N. Israelachvili. Intermolecular and surface forces. Academic Press, London, 1997.

[12] G. C. Maitland and E. B. Smith. Chemical Society Reviews, 2:181-201, 1973.

[13] J. O. Hirschfelder, C. F. Curtiss, and R. F. Bird. Molecular Theory of gases and liquids. Wiley, 1954.

[14] J. M. Prausnitz, R. N. Lichtenhaler, and de Azevedo Gomes. Termodinámica molecular de los equilibrios de fases. Prentice Hall, 2000.

[15] J. D. Jackson. Classical Electrodynamics. John Wiley and Sons, Inc, 1999.

[16] T. Kihara. Intermolecular Forces. John Wiley and Sons, New York, 1978.

[17] J. E. Lennard-Jones. Proc. Phys. Soc., 43:461-482, 1931.

[18] W. H. Stockmayer. J. Chem. Phys., 9:398-402, 1941.

[19] J. J. Weis and D. Levesque. J. Chem. Phys., 125:034504, 2006.

[20] G. N. Patey and J. P. Valleau. J. Chem. Phys., 61(2):534-540, 1974.

[21] K. Lucas. Molecular Models for fluids. Cambridge, University Press, 2006.

[22] N. M. Laurendeau. Statistical Thermodynamics. Fundamentals and applications. Cambridge, University Press, 2005.

[23] J. H. Dymond, K. N. Marsh, R. C. Wilhoit, and K. C. Wong. Virial Coefficients of Pure Gases and Mixtures, in: Landolt-Börnstein, Numerical Data and Functional Relationships in Science and Technology, in: M. Frenkel, K.N. Marsh (Eds.), New Series, Group IV: Physical Chemistry, volume 21. Springer-Verlag, 2002 .

[24] R. C. Reid, J. M. Prausnitz, and B. E. Poling. The properties of Gases and Liquids. McGraw-Hill, Inc., 1987.

[25] H. W. Xiang. The Corresponding-States Principle and its practice. Thermodynamic, Transport and Surface properties of fluids. Elsevier, 2005.

[26] K. E. Gubbins and T. M. Reed. Applied Statistical Mechanics. Thermodynamic and transport properties of fluids. McGraw-Hill, 1973.

[27] D. Cook and J. S. Rowlinson. Proc. R. Soc. Lond. A, 219:405-418, 1953.

[28] F. H. Stillinger. J. Chem. Phys., 117(1):288-296, 2002.

[29] M. P. Allen and D. J. Tildesley. Computer simulation of liquids. Clarendon Press Oxford, 1991.

[30] D. Frenkel and B. Smith. Understanding Molecular Simulation: From algorithms to application. Academic Press, 2002.

[31] M. P. Allen. Computing, 23(2):1-28, 2004.

[32] J. Bartke. Computer simulation of the Stockmayer fluid. Ph. D. Thesis, Bergische Universität Wuppertal, Germany, 2008.

[33] D. C. Rapaport. The art of molecular dynamics simulation. Cambridge University Press, 1998. 
[34] M. Abramowitz and I. A. Stegun. Handbook of Mathematical Functions with Formulas, Graphs, and Mathematical Tables. Dover, New York, 1972.

[35] J. H. Vlugt, J. P. J. M. van der Eerden, M. Dijkstra, B. Smit, and D. Frenkel. Introduction to Molecular Simulation and Statistical Thermodynamics. http://www.phys.uu.nl/ vlugt/imsst, 2008. [Delft, The Netherlands.].

[36] F. Ercolessi. Molecular dynamics primer. http://www.fisica.uniud.it/〜ercolessi/, 1997. [Dipartimento di Fisica Università di Udine.]. 


\section{Capítulo 3}

\section{Teoría para fluidos no conformales}

\subsection{Introducción}

Al final del primer capítulo se ha introducido el concepto de potencial efectivo, su aplicación a sistemas en la fase gaseosa y las posibles extensiones a densidades mayores. También se ha establecido cómo un conjunto de sustancias reales pueden representarse mediante una función potencial $\Phi=\epsilon f(r / \delta)$ única, ec. (2.25. pág. 15), con una elección adecuada de parámetros $\epsilon$ y $\delta$. Aquellas sustancias que cumplen ésta misma forma funcional se les conoce como fluidos conformales, siendo los gases nobles un ejemplo de ello, por lo que el término de no conformalidad será aplicado a aquellas sustancias que no cumplen este principio.

La teoría no conformal (NC) ha sido desarrollada como una extensión directa al principio de estados correspondientes (PEC) al introducir un parámetro molecular (extra a los parámetros comúnes de energía y distancia) que mide la no conformalidad entre los perfiles de un potencial de un sistema de interés y otro elegido como referencia. Dicho parámetro modifica el perfil del potencial, haciéndolo más o menos suave, respecto a una referencia y por ello se le ha asignado el nombre de "suavidad".

Estudios de una gran variedad de sistemas, tanto esféricos como no esféricos, muestran que tomando a la suavidad como un parámetro constante se obtienen resultados de alta exactitud. A tal aproximación se le da el nombre de teoría ANC la cual logra una relación lineal entre el segundo coeficiente virial $B(T)$ del sistema de estudio con el de la referencia $B_{0}(T)$. La importancia de la teoría ANC reside en su capacidad para reproducir, dentro del error experimental, las propiedades de más de 60 sustancias puras, y sus mezclas, en la fase gaseosa.

Al final del capítulo se presentan los detalles de cómo las cantidades que identifican al sistema ANC de referencia son obtenidas de manera analítica. Ellas son escritas en términos de funciones especiales (cilíndrico hiperbólicas, gamma, gamma incompletas, entre otras) haciéndonos contar con expresiones compactas cuyas principales ventajas y aplicaciones serán mencionadas en esta última sección.

\subsection{Teoría no conformal}

\subsubsection{Diámetros de colisión y fluidos equivalentes ${ }^{1}$}

Supóngase un sistema en fase gaseosa cuyas partículas interactúan por pares mediante el potencial $\Phi_{2}(r, \Omega)$, donde $r$ es la distancia entre las partículas y $\Omega$ su orientación relativa. Dicho potencial tiene un mínimo bien definido de profundidad $\Phi_{2}\left(\delta_{\Omega}, \Omega\right)=-\epsilon_{\Omega}$ en el punto $r=\delta_{\Omega}$ el cual separa al potencial en regiones repulsiva $\left(0 \leq r \leq \delta_{\Omega}\right)$ y atractiva $\left(r>\delta_{\Omega}\right)$; además, se supone que dicho potencial tiende a cero cuando $r \rightarrow \infty \mathrm{y}$ a infinito cuando $r \rightarrow 0$.

\footnotetext{
${ }^{1}$ Los principales aspectos de la teoría no conformal, y la forma aproximada, aquí expuestos pueden revisarse en las referencias $[1-4]$.
} 
Para el potencial $\Phi_{2}(r, \Omega)$ pueden calcularse diámetros medios en los que se registran las colisiones binarias y son obtenidas a partir de las frecuencias de colisión en el límite de bajas densidades. Estos diámetros medios de colisión son obtenidos a partir del promedio de todas las distancias de máxima aproximación entre los centros de las moléculas a una temperatura dada. Las colisiones repulsivas se dan en un nódulo con diámetro medio $\sigma$ dado por

$$
\sigma^{3}(T) \mathrm{e}^{\epsilon / k_{\mathrm{B}} T}=\int d \Omega \int_{0}^{\delta_{\Omega}} d r r^{3}\left(\frac{\partial \mathrm{e}^{-\Phi_{2}(r, \Omega) / k_{\mathrm{B}} T}}{\partial r}\right)
$$

y las colisiones atractivas por el intervalo medio de las fuerzas atractivas $R$ por

$$
R^{3}(T)\left(\mathrm{e}^{\epsilon / k_{\mathrm{B}} T}-1\right)=-\int d \Omega \int_{\delta_{\Omega}}^{\infty} d r r^{3}\left(\frac{\partial \mathrm{e}^{-\Phi_{2}(r, \Omega) / k_{\mathrm{B}} T}}{\partial r}\right)
$$

donde esta última cantidad también puede entenderse como el diámetro medio de un cascarón atractivo. La energía $\epsilon$ que aparece en estas ecuaciones se elige de tal forma que sea el mismo mínimo energético para todos los integrantes de la familia de potenciales $\Phi_{2}(r, \Omega)$ y el del potencial de pozo cuadrado representado por el lado izquierdo de las ecuaciones 3.1) y 3.2.

Puede demostrarse que, para una temperatura dada, tanto $\sigma$ como $R$ corresponden respectivamente al diámetro y alcance de un fluido equivalente de pozo cuadrado que tiene exactamente la misma presión que el sistema de interés [1]. Efectivamente, de 2.20 pág. 13, 3.1) y (3.2) se observa que

$$
B(T)=\frac{2 \pi}{3}\left[\sigma^{3}(T) \mathrm{e}^{\epsilon / k_{\mathrm{B}} T}-R^{3}(T)\left(\mathrm{e}^{\epsilon / k_{\mathrm{B}} T}-1\right)\right]
$$

el cual es ideńtico al segundo coeficiente virial de un pozo cuadrado (SW) de profundidad $\epsilon$, diámetro $\sigma$ y alcance $R$. El alcance efectivo de este potencial SW es entonces

$$
\lambda_{\mathrm{ef}}=\frac{R(T)}{\sigma(T)} .
$$

Por conveniencia, en la ec. 3.3 se definen parámetros de escala en términos de las propiedades del sistema de interés. Para manejar definiciones convenientes se integran por partes las ecs. (3.1) y (3.2) tal que

$$
\sigma^{3}(T) \mathrm{e}^{\epsilon / k_{\mathrm{B}} T}=\int d \Omega \delta_{\Omega}^{3} \mathrm{e}^{\epsilon_{\Omega} / k_{\mathrm{B}} T}-3 \int d \Omega \int_{0}^{\delta_{\Omega}} d r r^{2} \mathrm{e}^{-\Phi_{2}(r, \Omega) / k_{\mathrm{B}} T}
$$

y

$$
R^{3}(T)\left(\mathrm{e}^{\epsilon / k_{\mathrm{B}} T}-1\right)=\int d \Omega \delta_{\Omega}^{3}\left(\mathrm{e}^{\epsilon_{\Omega} / k_{\mathrm{B}} T}-1\right)+3 \int d \Omega \int_{\delta_{\Omega}}^{\infty} d r r^{2}\left(\mathrm{e}^{-\Phi_{2}(r, \Omega) / k_{\mathrm{B}} T}-1\right)
$$

donde $\sigma$ es similar al diámetro de esferas duras, a bajas densidades, en la teoría de perturbación WCA. Basándonos en éstas dos ecuaciones se introducen las definiciones del diámetro medio $\delta$ de la molécula y el de la energía $\epsilon$ promediando angularment $\AA^{2} \operatorname{los}$ respectivos parámetros de los potenciales $\Phi_{2}(r, \Omega)$

$$
\begin{aligned}
\delta^{3} & \equiv \int d \Omega \delta_{\Omega}^{3} \\
\mathrm{e}^{\epsilon / k_{\mathrm{B}} T} & \equiv \frac{\int d \Omega \delta_{\Omega}^{3} \mathrm{e}^{\epsilon_{\Omega} / k_{\mathrm{B}} T}}{\int d \Omega \delta_{\Omega}^{3}}
\end{aligned}
$$

que en el caso del diámetro para moléculas esféricas, es exactamente la distancia centro-a-centro entre ellas a la cual $\Phi_{2}$ tiene su mínimo; difiere del diámetro de van der Waals $\delta_{\mathrm{vdw}}$ definido como la distancia a la cual la energía es cero, i.e. $\Phi_{2}\left(r=\delta_{\mathrm{vdw}}\right)=0$.

Con éstos dos nuevos parámetros las ecuaciones 3.5 y 3.6 pueden reescribirse como

\footnotetext{
${ }^{2}$ La definición de energía incluida aquí es distinta a la dada originalmente en [3].
} 


$$
\sigma^{3}(T)=\delta^{3}-\frac{3}{\mathrm{e}^{\epsilon / k_{\mathrm{B}} T}} \int d \Omega \int_{0}^{\delta_{\Omega}} d r r^{2} \mathrm{e}^{-\Phi_{2}(r, \Omega) / k_{\mathrm{B}} T}
$$

y

$$
R^{3}(T)=\delta^{3}+\frac{3}{\mathrm{e}^{\epsilon / k_{\mathrm{B}} T}-1} \int d \Omega \int_{\delta_{\Omega}}^{\infty} d r r^{2}\left(\mathrm{e}^{-\Phi_{2}(r, \Omega) / k_{\mathrm{B}} T}-1\right)
$$

\subsubsection{Volúmenes reducidos de colisión}

Tomando como unidades de longitud y energía a las cantidades $\delta$ y $\epsilon$ ya antes mencionadas y definiendo las siguientes cantidades: $y=r / \delta, y_{\Omega}=\delta_{\Omega} / \delta, \Phi^{*}=\Phi_{2} / \epsilon$ y $T^{*}=k_{\mathrm{B}} T / \epsilon$, pueden reducirse los diámetros de colisión. Con esto el volumen repulsivo se escribe

$$
b^{*}\left(T^{*}\right)=\frac{\sigma^{3}(T)}{\delta^{3}(T)}=1-\frac{3}{e^{1 / T^{*}}} \int d \Omega \int_{0}^{y_{\Omega}} d y y^{2} \mathrm{e}^{-\Phi^{*}(y, \Omega) / T^{*}}
$$

y el volumen reducido encerrado por el cascarón atractivo será

$$
\Lambda^{*}\left(T^{*}\right)=\frac{R^{3}(T)}{\delta^{3}(T)}=1+\frac{3}{\left(\mathrm{e}^{1 / T^{*}}-1\right)} \int d \Omega \int_{y_{\Omega}}^{\infty} d y y^{2}\left(\mathrm{e}^{-\Phi^{*}(y, \Omega) / T^{*}}-1\right) .
$$

El segundo coeficiente virial del potencial reducido para $\Phi(y, \Omega)$ queda en la forma final

$$
B^{*}\left(T^{*}\right)=\frac{B(T)}{2 \pi \delta^{3} / 3}=b^{*}\left(T^{*}\right) \mathrm{e}^{1 / T^{*}}-\Lambda^{*}\left(T^{*}\right)\left(\mathrm{e}^{1 / T^{*}}-1\right)
$$

con alcance del potencial SW equivalente dado por

$$
\lambda_{\mathrm{ef}}^{3}=\frac{\Lambda^{*}\left(T^{*}\right)}{b^{*}\left(T^{*}\right)} .
$$

\subsubsection{Función suavidad}

Los volúmenes reducidos 3.11 y 3.12 pueden ser reescritos en una manera más conveniente para detallar la forma del perfil del potencial mediante la transformación $\phi(y, \Omega)=\Phi_{2}(y, \Omega) / \epsilon_{\Omega}+1$, obteniéndose así las expresiones

$$
b^{*}\left(T^{*}\right)=1+\int d \Omega \int_{1}^{\infty} d \phi\left[\frac{\partial y^{3}(\phi, \Omega)}{\partial \phi}\right]_{\mathrm{R}} \mathrm{e}^{-\phi / T^{*}}
$$

y

$$
\Lambda^{*}(T)=1+\frac{1}{e^{1 / T^{*}}-1} \int d \Omega \int_{0}^{1} d \phi\left[\frac{\partial y^{3}(\phi, \Omega)}{\partial \phi}\right]_{\mathrm{A}}\left(\mathrm{e}^{-(\phi-1) / T^{*}}-1\right)
$$

donde $y(\phi, \Omega)$ es el inverso de $\phi(y, \Omega)$ en las regiones repulsiva y atractiva de $\phi(y)$ denotado por los índices $\mathrm{R}$ y A, respectivamente. La ausencia de conformalidad entre $\Phi_{2}(y, \Omega)$ y un potencial de referencia, $\Phi_{0}(y)$, de igual profundidad $\epsilon$, es considerada por la función suavidad $s(\phi, \Omega)$ definida como

$$
s(\phi, \Omega)=\frac{\epsilon_{\Omega}\left[\partial \Phi_{0}(y) / \partial y^{3}\right]_{y_{0}(\phi)}}{\epsilon\left[\partial \Phi_{2}(y, \Omega) / \partial y^{3}\right]_{y(\phi)}},
$$

donde las derivadas de la ecuación (3.17) son evaluadas para el mismo valor de la energía reducida $\phi$. De esta ecuación es claro que el parámetro de forma $s$ mide la razón de pendientes entre los potenciales $\Phi_{2}$ y $\Phi_{0}$ para una $y$ dada. Así, por definición, si $s<1$ el potencial $\Phi_{2}$ tiene una pendiente más pronunciada que $\Phi_{0}$. En general, el cambio del perfil respecto a la referencia es distinto en ambos lados del potencial $\Phi_{2}$ de tal forma que se definen dos parámetros de suavidad tanto para la parte repulsiva, $s_{\mathrm{R}}$, como para la atractiva, $s_{\mathrm{A}}$. 


\subsection{Teoría no conformal aproximada}

\subsubsection{Potencial ANC}

En una gran cantidad de casos [2] las suavidades $s_{\mathrm{R}}$ y $s_{\mathrm{A}}$ son independientes de $\phi$ y por lo tanto pueden tomarse constantes tales que

$$
S_{\mathrm{R}}=\left\langle s_{\mathrm{R}}\right\rangle_{\Omega}
$$

y

$$
S_{\mathrm{A}}=\left\langle s_{\mathrm{A}}\right\rangle_{\Omega} .
$$

Mediante esta aproximación se logra una definición única de la suavidad para cada uno de las ramas del potencial $\Phi_{2}$. La simplificación al marco anterior se le asigna el nombre de teoría no-conformal aproximada (ANC) y su potencial característico por pares toma la forma

$$
\frac{\Phi_{\mathrm{ANC}}\left(y, S_{i}\right)}{\epsilon}=\left(\frac{1-a}{\chi\left(y, S_{i}\right)-a}\right)^{12}-2\left(\frac{1-a}{\chi\left(y, S_{i}\right)-a}\right)^{6}
$$

con

$$
\chi\left(y, S_{i}\right)^{3}=\frac{y^{3}-1}{S_{i}}+1
$$

donde $S_{i}=S_{\mathrm{R}}$ cuando $y \leq 1$ y $S_{i}=S_{\mathrm{A}}$ cuando $y>1$. Así, el potencial ANC está caracterizado por los parámetros moleculares de distancia $\delta_{\mathrm{ANC}}$ (el cual corresponde con el mínimo local), de energía $\epsilon_{\mathrm{ANC}}$ (la energía en el mismo punto) y los respectivos parámetros de suavidad $S_{\mathrm{R}}$ y $S_{\mathrm{A}}$. Obsérvese que este potencial tiene un nódulo duro dado por $y_{0}=\left[1+S_{\mathrm{R}}\left(a^{3}-1\right)\right]^{1 / 3}$ dependiente de la suavidad repulsiva.

Cuando $S=1.0$ se tiene que $\chi=y$ por lo que el potencial ANC se reduce a la forma

$$
\frac{\Phi_{0}(y)}{\epsilon}=\left(\frac{1-a}{y-a}\right)^{12}-2\left(\frac{1-a}{y-a}\right)^{6}
$$

el cual es un potencial tipo Kihara esférico 12-6, ec. 2.15), donde a es el diámetro del nódulo duro en unidades de $\delta_{\text {ANC }}$ y se toma como potencial de referencia. Además, si se toma el valor de $a=0.0957389$ se tiene que $\Phi_{0}(y)$ es una buena representación del potencial por pares del argón [2]. Un caso especial se da cuando $S \cong 1.13$, valor con el cual el potencial ANC correspondiente es prácticamente conformal a la función LJ 12-6 [5].

\subsubsection{Segundo coeficiente virial ANC}

A partir de la forma de $\Phi_{\text {ANC }}$ se puede demostrar formalmente [3] que los volúmenes dados por las expresiones (3.15) y 3.16 se convierten en

$$
b^{*}\left(T^{*}\right)=1-S_{\mathrm{R}}+S_{\mathrm{R}} b_{0}^{*}\left(T^{*}\right)
$$

y

$$
\Lambda^{*}\left(T^{*}\right)=1-S_{\mathrm{A}}+S_{\mathrm{A}} \Lambda_{0}^{*}\left(T^{*}\right)
$$

donde $b_{0}^{*}\left(T^{*}\right)$ y $\Lambda_{0}^{*}\left(T^{*}\right)$ son los volúmenes de referencia para el potencial $\Phi_{0} 3^{3}$

Las expresiones 3.23 y 3.24 nos permiten escribir, en una forma simple, el segundo coeficiente virial, $B^{*}\left(T^{*}\right)$, en términos de los volúmenes efectivos de referencia

$$
B^{*}\left(T^{*}\right)=\left[1-S_{\mathrm{R}}+S_{\mathrm{R}} b_{0}^{*}\left(T^{*}\right)\right] \mathrm{e}^{1 / T^{*}}-\left[1-S_{\mathrm{A}}+S_{\mathrm{A}} \Lambda_{0}^{*}\left(T^{*}\right)\right]\left\{\mathrm{e}^{1 / T^{*}}-1\right\} .
$$

\footnotetext{
${ }^{3}$ Una manera independiente para llegar a estas mismas expresiones, partiendo del potencial ANC, ha sido elaborada como parte del presente trabajo y pueden observarse esos resultados al final de este capítulo.
} 


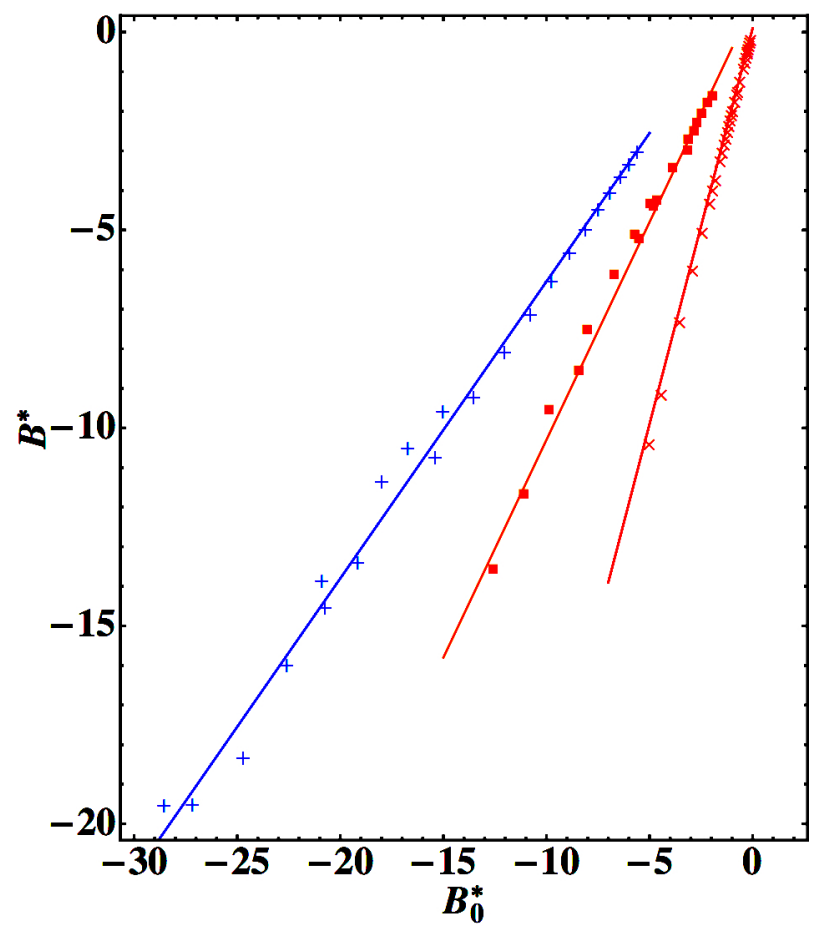

Figura 3.1: Comportamiento lineal entre segundos coeficientes viriales experimentales [6] para algunas sustancias simples con respecto a la referencia: $\mathrm{H}_{2} \mathrm{O}(+), \mathrm{HCl}(\boldsymbol{\square})$ y $\mathrm{Ar}(\times)$. Las líneas rectas han sido dibujadas para ayudar a la vista.

Un caso importante se da cuando $S=S_{\mathrm{R}}=S_{\mathrm{A}}$ lo que simplifica la presentación de $B^{*}\left(T^{*}\right)$ obteniéndose una relación lineal entre los segundos coficientes viriales del fluido de estudio y el de referencia

$$
B^{*}\left(T^{*}\right)=1-S+S B_{0}^{*}\left(T^{*}\right)
$$

La forma lineal que presentan los volúmenes de colisión con sus respectivos volúmenes de referencia, ecs. 3.23 y 3.24 y en especial esta última ecuación han sido una fuente intensa de estudios de sistemas no conformales. Ambas expresiones 3.25 y 3.26 son consideradas como una extensión al PEC al relacionar una propiedad termodinámica entre sistemas no-conformales de acuerdo a sus potenciales $\Phi_{\text {ANC }}$ con respecto a un potencial de referencia $\Phi_{0}$. Muchos segundos coeficientes viriales de sustancias reales siguen el comportamiento mostrado por la ecuación (3.26) con respecto al segundo coeficiente virial de una sustancia elegida como referencia; en la figura 3.1 se muestran algunos ejemplos experimentales de dicho comportamiento.

Con el fin de identificar adecuadamente la situación al usar una o dos suavidades en el potencial ANC, decimos que cuando $S=S_{\mathrm{R}}=S_{\mathrm{A}}$ se está trabajando con la versión 1S de la teoría ANC; mientras que cuando se tiene que $S_{\mathrm{R}} \neq S_{\mathrm{A}}$ la versión que se maneja es la $2 \mathrm{~S}$; la mayor parte de sistemas reales a los cuales se les ha aplicado esta teoría hacen uso de la primera versión con excelentes resultados.

El potencial ANC, como ya se mencionó arriba, tiene la característica de que su perfil se modifica variando el término $S_{\mathrm{R}}$ ó $S_{\mathrm{A}}$ del potencial. En la figura 3.2 se presenta un conjunto de potenciales de esta familia para el caso $1 \mathrm{~S}$, mostrando cómo el pozo de $\Phi_{\mathrm{ANC}}$ se ensancha a medida que el parámetro $S$ se incrementa. Si dos interacciones $\Phi_{\text {ANC }}^{(1)}$ y $\Phi_{\text {ANC }}^{(2)}$ tienen la misma suavidad ambas funciones son conformales y se superponen una a la otra cumpliendo el PEC. 


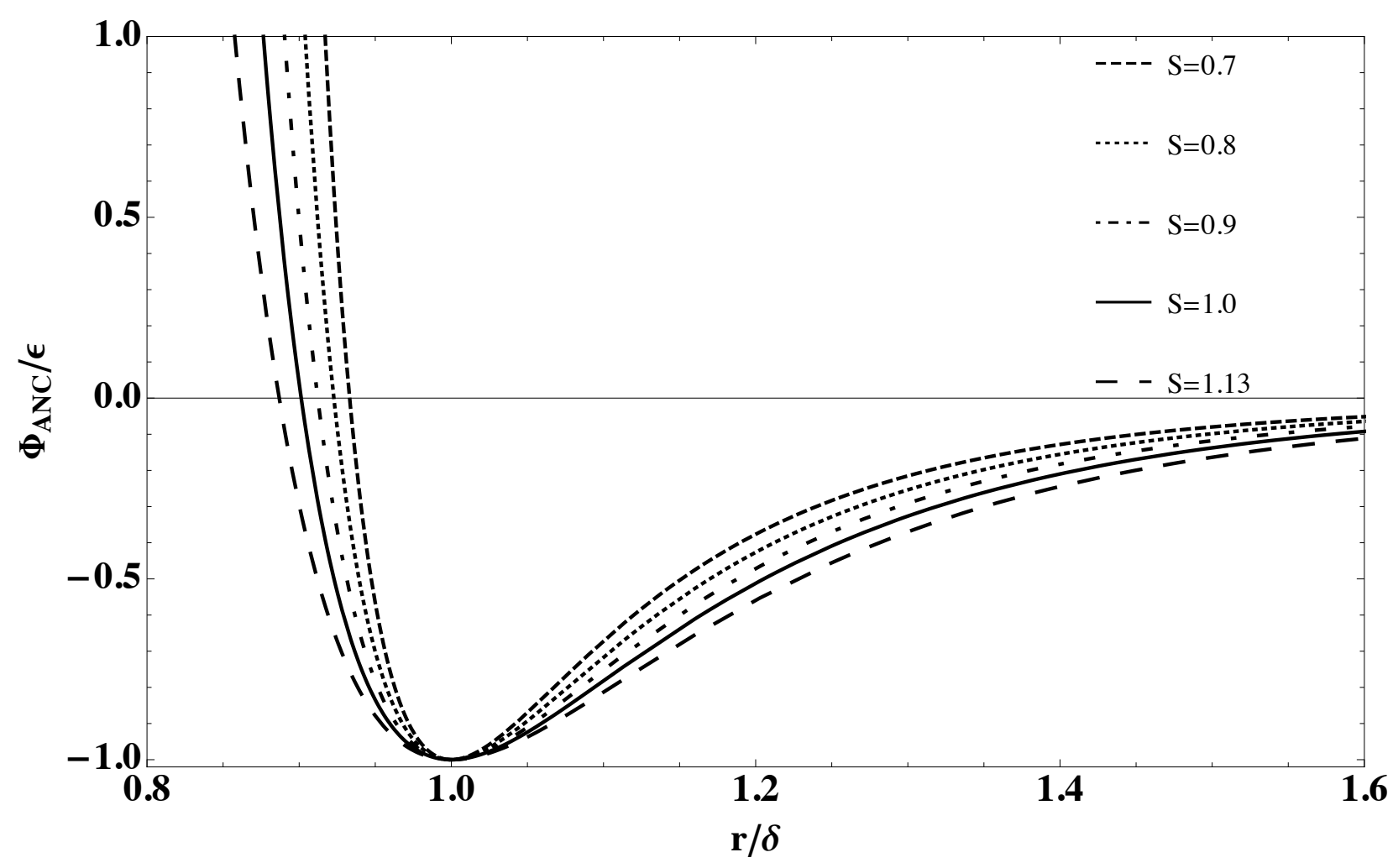

Figura 3.2: Perfil del potencial ANC 1-S para distintas suavidades.

\subsubsection{Volúmenes de referencia}

La incorporación de un modelo ANC para el estudio de sistemas reales (o realistas) implica el conocimiento exacto de las integrales $b_{0}^{*}$ y $\Lambda_{0}^{*}$ para aquellas temperaturas en las cuales se desee trabajar. Existen dos formas de calcular dichos volúmenes y ambas han sido ocupadas en este trabajo. La primera de ellas se presenta como una serie finita que fácilmente puede incluirse en cualquier rutina computacional para dos intervalos de $T^{*}$. Para $0.05<T^{*}<0.4$ :

$$
\begin{aligned}
b_{0}^{*}\left(T^{*}\right) & =\sum_{m=0}^{5} c_{m}\left(T^{*}\right)^{m} \\
\Lambda_{0}^{*}\left(T^{*}\right) & =b_{0}^{*}\left(T^{*}\right) \sum_{m=0}^{5} l_{m}\left(T^{*}\right)^{m}
\end{aligned}
$$

y para $0.4<T^{*}<250$ :

$$
\begin{aligned}
b_{0}^{*}\left(T^{*}\right) & =\sum_{m=0}^{\mathrm{j}} c_{m}\left(\ln T^{*}\right)^{m} \\
\Lambda_{0}^{*}\left(T^{*}\right) & =b_{0}^{*}\left(T^{*}\right) \sum_{m=0}^{\mathrm{k}} l_{m}\left(\ln T^{*}\right)^{m}
\end{aligned}
$$

donde las constantes $c_{m}$ y $l_{m}$ para ambos intervalos de temperatura y demás información pueden revisarse en las referencias $[4,5,7]$.

La segunda forma de obtener $b_{0}^{*}$ y $\Lambda_{0}^{*}$ consiste en una serie semianalítica ligada a aquellas expresiones matemáticas encontradas regularmente en el estudio de fluidos y se muestra al final del capítulo como un resultado más de este trabajo. 


\subsubsection{Tercer coeficiente virial ANC}

Para un fluido ANC se ha construido un modelo para el tercer coeficiente virial el cual se basa en la suma de dos contribuciones y la dependencia de la no conformalidad en ellas. La primera contribución es debida a las interacciones binarias y representada por un potencial ANC, y la otra contribución surge de las fuerzas entre tres cuerpos representadas por el potencial Axilrod-Teller-Mutto [8]. Dicha interacción, ademas de incluir la configuración relativa entre las tres partículas, incluye el parámetro $\nu$ ATM (en ocasiones denotado por $C_{9}$ ) el cual gobierna la intensidad de las fuerzas ternarias.

La expresión para el tercer coeficiente ANC $+\mathrm{ATM}$, en su forma reducida, se escribe como

$$
C_{\mathrm{ANC}}^{*}=C_{2}^{*}\left(T^{*}, S\right)+C_{3}^{*}\left(T^{*}, S, \nu^{*}\right)
$$

donde $C_{2}^{*}$ y $C_{3}^{*}$ están dados por

$$
C_{2}^{*}=K_{0}(S)+3 K_{1}(S) x+3 K_{2}(S) x^{2}+K_{3}(S) x^{3}
$$

y

$$
C_{3}^{*}=\nu^{*} \mathcal{Q}\left(T^{*}, S\right)
$$

donde $x=\mathrm{e}^{1 / T^{*}}-1$, y $\mathcal{Q}$ es una integral múltiple obtenida de desarrollar la expresión completa del tercer coeficiente virial en potencias de $\nu^{*}=\nu / \epsilon \delta^{9}$. Tanto las funciones $K_{i}(S)$ como $\mathcal{Q}$ son obtenidas por integración numérica, ajustadas usando polinomios en $x$ y cuya forma exacta puede ser revisada en la fuente original [9].

\subsubsection{Fluidos ANC densos}

Mediante simulaciones de Monte Carlo - Gibbs ensamble (GEMC) ha sido calculado el equilibrio líquidovapor para un intervalo de suavidades de: $S \in[0.6,1.0]$. El comportamiento de la curva ortobárica muestra que a medida que la suavidad aumenta el domo también se eleva hacia temperaturas mayores. Los parámetros críticos del fluido ANC hallados muestran una función única con la suavidad y se pueden representar de manera aproximada por polinomios en $S$ como [10-12]

$$
\begin{aligned}
& T_{\mathrm{c}, \mathrm{ANC}}^{*}(S)=0.348915+0.326038 S+0.443771 S^{2} \\
& \rho_{\mathrm{c}, \mathrm{ANC}}^{*}(S)=0.447+0.0084 S \\
& P_{\mathrm{c}, \mathrm{ANC}}^{*}(S)=-0.0108+0.1572 S .
\end{aligned}
$$

\subsection{El potencial ANC como modelo efectivo}

La teoría efectiva ANC ha sido aplicada a una gran variedad de sistemas (tanto teóricos como reales) puros y sus mezclas, con excelentes resultados abarcando estados que van desde fases diluidas hasta fluidos densos cerca del punto triple e incluso, en algunos casos, hasta diez veces la temperatura crítica. En la práctica, los sistemas estudiados se consideran que están en fase isotrópica y además se desprecian los posibles grados internos de libertad. Debido a que la virtud principal de esta teoría radica en que el potencial ANC es escrito en función de las características moleculares del fluido estudiado, ella cae dentro del campo de la termodinámica molecular [13].

Así, se busca un potencial esférico del tipo $\Phi_{\mathrm{ANC}}$, ec. (3.20), que reproduzca de manera eficiente la interacción $\Phi$, ec. 2.5 tal que aquellas propiedades que caracterizan a la sustancia de estudio tales como la interacción electrostática, la forma de la molécula, fuerzas de muchos cuerpos, etc, son incorporados en los parámetros efectivos ANC: $\epsilon, \delta, S_{\mathrm{R}}$ y $S_{\mathrm{A}} 4^{4}$ Es decir, cualquier propiedad termodinámica del fluido tal como la presión,

\footnotetext{
${ }^{4}$ En el caso de mezclas de sustancias se introducen términos cruzados adicionales para cada uno de los parámetros del potencial ANC $[5,7,14-16]$.
} 
dependerá de las variables termodinámicas (densidad, temperatura y fracción molar) y funcionalmente del conjunto de parámetros ANC.

A nivel del gas, un potencial efectivo ANC con parámetros constantes es suficiente para reproducir $B(T)$ (dentro del error experimental) de muchas sustancias de naturaleza sencilla. En muchas ocasiones sólo se requiere el uso de un parámetro único de suavidad (aproximación 1S). Con esta aproximación y mediante la inversión de datos experimentales de $B(T)$ se han obtenido potenciales efectivos tipo ANC para gases nobles y sus mezclas [5], los halometanos [11], moléculas homodiatómicas y sus mezclas [14], e incluso con moléculas lineales pequeñas con multipolos permanentes [15]. Todos estos resultados mejoran a los generados por otros modelos como el LJ 12-6, el Kihara o aquellos obtenidos a partir de correlaciones empíricas según las referencias antes citadas.

Cuando la complejidad de la molécula es mayor (o a una mayor densidad) el potencial requiere generalmente que los parámetros dependan del estado del fluido. En este caso se busca que dichos parámetros tengan variaciones suaves y pequeñas sobre amplios rangos de temperatura y densidad, mientras se mantiene una buena exactitud con el modelo. Dos ejemplos con esta nueva aproximación son de primera importancia para la investigación actual: A) El estudio de moléculas convexas ha logrado vincular los parámetros ANC con las medidas fundamentales del cuerpo y se ha aplicado a familias de moléculas reales tales como los $n$-alcanos y los $n$-perfluoroalcanos $[4,17]$; y B) en el caso de interacciones dipolo-dipolo, con intensidades bajas, la aplicación del modelo ANC permite la inclusión del mismo parámetro suavidad tanto en la zona repulsiva del potencial como en la atractiva (aproximación 1S), que junto con el resto de los parámetros moleculares tendrán una dependencia explícita en la temperatura del sistema [18]. Dicha dependencia es muy marcada en el parámetro energético y muy pequeña para el de la longitud por lo que esta última puede considerarse constante como una primera aproximación. Los resultados también son aplicados a sustancias reales, como el $\mathrm{HCl}$, con intensidades dipolares pequeñas.

El comportamiento del tercer coeficiente virial ANC con respecto a la temperatura puede obtenerse con el modelo ANC-ATM y las predicciones hechas para sustancias reales concuerdan con los datos reportados en la literatura dentro de los intervalos permitidos del error experimental [19].

Entre otras sustancias estudiadas con el modelo ANC se encuentran algunos gases nobles: argón y kriptón, moléculas diatómicas: $\mathrm{N}_{2}, \mathrm{~F}_{2}$ y CO, el perfluorometano y el eteno [20]. Además, el potencial ANC ha mostrado su eficacia en la reproducción del segundo coeficiente virial acústico de nueve sustancias reales [21], los coeficientes de viscosidad $\eta$ para los gases nobles más pesados, moléculas diátomicas y algunas poliatómicas [22] y la conductividad térmica para el caso de los gases nobles [23].

\subsection{Volúmenes de referencia ANC en forma analítica}

Durante el desarrollo de la investigación, nuevas expresiones analíticas se han obtenido para los volúmenes de referencia ANC. A continuación se presenta la metodología seguida y la forma final de dichos volúmenes. Todos los cálculos realizados para obtener dichas cantidades pueden revisarse en el apéndice B.

Como antecedente inmediato, la forma analítica del segundo coeficiente virial de un fluido ANC-1S está dada por

$$
B_{0}^{*}=B_{\mathrm{ANC}-1 \mathrm{~S}}^{*}(S=1)=a^{3}+3(1-a)\left[a^{2} \mathcal{F}_{1}\left(T^{*}\right)+a(1-a) \mathcal{F}_{2}\left(T^{*}\right)+\frac{(1-a)^{2}}{3} \mathcal{F}_{3}\left(T^{*}\right)\right]
$$

donde las funciones $\mathcal{F}_{k}\left(T^{*}\right)$ son ampliamente conocidas en el ámbito de la teoría de cuerpos convexos [24-27], apéndice A.2.1. El desarrollo para obtener la expresión (3.37) del segundo coeficiente virial de un potencial ANC se puede hallar en la referencia [28] y una metodología similar ha sido aplicada en el cálculo de los volúmenes de referencia de la teoría ANC. 


\subsubsection{Nueva forma cerrada para la integral $\mathcal{F}_{k}\left(T^{*}\right)$}

Antes de continuar con los volúmenes de colisión, mostramos una simplificación a la forma cerrada de la integral $\mathcal{F}_{k}(T)$, ecuación A.19 pág. 107). Usando las fórmulas de recurrencia de las funciones cilíndricoparabólicas $D_{y}[x][29,30]$, la expresión $($ A.19] se logra escribir en una forma mucho más compacta:

$$
\mathcal{F}_{k}(T)=\left(\frac{2 \epsilon}{k_{\mathrm{B}} T}\right)^{\frac{k}{12}} \Gamma\left[\frac{6-k}{6}\right] \exp \left[\frac{\epsilon}{2 k_{\mathrm{B}} T}\right] D_{\frac{k}{6}}\left[-\left(\frac{2 \epsilon}{k_{\mathrm{B}} T}\right)^{\frac{1}{2}}\right] .
$$

El cálculo completo se encuentra en el apéndice B.1 Esta nueva forma de la expresión $\mathcal{F}_{k}(T)$ aporta, además de una reducción en las rutinas dentro de cualquier software matemático, un elemento más a favor de aquellos sistemas que empleen este tipo de funciones.

\subsubsection{Volúmenes de colisión ANC}

Por definición, los volumenes de colisión reducidos tanto repulsivo $b^{*}\left(T^{*}\right)$, como atractivo $\Lambda^{*}\left(T^{*}\right)$, ecs. (3.11) y 3.12 , son

$$
\begin{aligned}
b^{*}\left(T^{*}\right) & =1-\frac{3}{\mathrm{e}^{1 / T^{*}}} \int_{0}^{1} \mathrm{e}^{-\Phi_{\mathrm{ANC}-2 \mathrm{~S}}^{*} / T^{*}} y^{2} d y \\
\Lambda^{*}\left(T^{*}\right) & =1+\frac{3}{\mathrm{e}^{1 / T^{*}}-1} \int_{1}^{\infty}\left(\mathrm{e}^{-\Phi_{\mathrm{ANC}-2 \mathrm{~S}}^{*} / T^{*}}-1\right) y^{2} d y .
\end{aligned}
$$

en donde se hace énfasis en el uso de un potencial ANC versión 2S, ec. 3.20 , y donde se ha considerado que $y=r / \delta_{\mathrm{ANC}}, T^{*}=k T / \epsilon_{\mathrm{ANC}} \mathrm{y} \Phi_{\mathrm{ANC}}^{*}=\Phi_{\mathrm{ANC}} / \epsilon_{\mathrm{ANC}}$.

A partir de la definición de la función $\chi$, ec. (3.21), y empleando el siguiente cambio de variable

$$
Y=\frac{\chi-a}{1-a}
$$

se tiene $q u \Phi^{5}$

$$
y^{3}=1-S_{i}+S_{i} \chi^{3} \quad \text { y } \quad S_{i} \chi^{2} d \chi=y^{2} d y
$$

el cual permite escribir a las integrales que aparecen en los volúmenes de colisión como

$$
\int_{0}^{1} \mathrm{e}^{-\Phi_{\mathrm{ANC}-2 \mathrm{~S}}^{*} / T^{*}} y^{2} d y=S_{\mathrm{R}}(1-a)\left[a^{2} \mathcal{D}_{1}\left(T^{*}\right)+a(1-a) \mathcal{D}_{2}\left(T^{*}\right)+\frac{(1-a)^{2}}{3} \mathcal{D}_{3}\left(T^{*}\right)\right]
$$

y

$$
\int_{1}^{\infty}\left(\mathrm{e}^{-\Phi_{\mathrm{ANC}-2 \mathrm{~S}}^{*} / T^{*}}-1\right) y^{2} d y=S_{\mathrm{A}}(1-a)\left[a^{2} \mathcal{E}_{1}\left(T^{*}\right)+a(1-a) \mathcal{E}_{2}\left(T^{*}\right)+\frac{(1-a)^{2}}{3} \mathcal{E}_{3}\left(T^{*}\right)\right] .
$$

donde las integrales $\mathcal{D}_{k}\left(T^{*}\right)$ y $\mathcal{E}_{k}\left(T^{*}\right)$ están escritas en términos de un potencial LJ 12-6 y pueden resolverse de forma analítica como una serie infinita de funciones gamma incompletas, dadas por las expresiones

$$
\begin{aligned}
\mathcal{D}_{k}\left(T^{*}\right) & =\frac{k}{12} \sum_{j=0}^{\infty} \frac{2^{j}}{j !} \Gamma\left[\frac{6 j-k}{12}, \frac{1}{T^{*}}\right] T^{*-\frac{6 j+k}{12}} \\
\mathcal{E}_{k}\left(T^{*}\right) & =1+\frac{k}{12} \sum_{j=0}^{\infty} \frac{2^{j}}{j !} \gamma\left[\frac{6 j-k}{12}, \frac{1}{T^{*}}\right] T^{*-\frac{6 j+k}{12}}
\end{aligned}
$$

con $k=1,2$ y 3, y cuya integración se realiza en el apéndice B.2.2

\footnotetext{
${ }^{5}$ En este contexto la nueva variable $Y$ es la posición reducida $(Y=r / \epsilon)$ de un potencial Lennard-Jones, por lo que la ecuación 3.39 es una relación lineal directa entre el cubo de las distancias reducidas entre un sistema ANC y uno LJ, haciendo de la suavidad el parámetro que las relaciona.
} 


\subsubsection{Volúmenes de colisión - referencia}

Sustituyendo $S=1$ en las ecuaciones B.4 y , B.5 se obtienen los volúmenes de referencia

$$
b_{0}^{*}\left(T^{*}\right)=1-\frac{3(1-a)}{\mathrm{e}^{1 / T^{*}}}\left[a^{2} \mathcal{D}_{1}\left(T^{*}\right)+a(1-a) \mathcal{D}_{2}\left(T^{*}\right)+\frac{(1-a)^{2}}{3} \mathcal{D}_{3}\left(T^{*}\right)\right]
$$

y

$$
\Lambda_{0}^{*}\left(T^{*}\right)=1+\frac{3(1-a)}{\mathrm{e}^{1 / T^{*}}-1}\left[a^{2} \mathcal{E}_{1}\left(T^{*}\right)+a(1-a) \mathcal{E}_{2}\left(T^{*}\right)+\frac{(1-a)^{2}}{3} \mathcal{E}_{3}\left(T^{*}\right)\right]
$$

cuyas definiciones permiten llegar a las ecuaciones 3.23 y 3.24 . Como era de esperarse, cuando $S_{\mathrm{R}}=S_{\mathrm{A}}$ este conjunto de ecuaciones se reducen al caso $1 \mathrm{~S}$.

\subsubsection{Caso especial ANC-LJ}

Un punto interesante a notar es aquella variante obtenida cuando se considera $a=0$ en la teoría ANC. El potencial ANC, ecuación (3.20), toma la siguiente forma

$$
\frac{\Phi_{\mathrm{ANC}}^{\prime}\left(y, S_{i}\right)}{\epsilon}=\left[\frac{S_{i}}{y^{3}-1+S_{i}}\right]^{4}-2\left[\frac{S_{i}}{y^{3}-1+S_{i}}\right]^{2},
$$

donde el símbolo prima hace referencia a este caso especial. La ventaja de este modelo consiste en que el potencial LJ 12-6 es el potencial de referencia, $\Phi_{\text {ANC }}^{\prime}\left(y, S_{i}=1\right)=\Phi_{0}^{\prime}=\Phi_{\mathrm{LJ}}$, por lo que

$$
B_{0}^{* \prime}=B_{\mathrm{LJ}}^{*}
$$

Esto último significa que el segundo coeficiente virial de cualquier fluido ANC-1S (con $a=0)$ puede escribirse en términos del segundo coeficiente virial de un fluido Lennard-Jones como

$$
B^{* \prime}\left(T^{*}\right)=1-S+S B_{\mathrm{LJ}}^{*}\left(T^{*}\right)
$$

cuya expresión analítica es bien conocida.

Para el caso 2S, usando $a=0$ en los volúmenes de referencia 3.44 y 3.45 , éstos se simplifican obteniéndose expresiones mucho más compactas

$$
\begin{aligned}
b_{0}^{* \prime}\left(T^{*}\right) & =1-\frac{\mathcal{D}_{3}\left(T^{*}\right)}{\mathrm{e}^{1 / T^{*}}}, \\
\Lambda_{0}^{* \prime}\left(T^{*}\right) & =1+\frac{\mathcal{E}_{3}\left(T^{*}\right)}{\mathrm{e}^{1 / T^{*}}-1},
\end{aligned}
$$

y ya que las relaciones lineales entre los volúmenes de colisión, $b^{*}$ y $\Lambda^{*}$, con sus respectivos volúmenes de referencia (ecs. 3.49 3.50 se mantienen, el segundo coeficiente virial ANC 2S estará dado por

$$
\begin{aligned}
B^{* \prime}\left(T^{*}\right) & =\left[1-S_{\mathrm{R}}+S_{\mathrm{R}} b_{0}^{* \prime}\left(T^{*}\right)\right] \mathrm{e}^{1 / T^{*}}-\left[1-S_{\mathrm{A}}+S_{\mathrm{A}} \Lambda_{0}^{* \prime}\left(T^{*}\right)\right]\left(\mathrm{e}^{1 / T^{*}}-1\right) \\
& =1-S_{\mathrm{R}} \mathcal{D}_{3}\left(T^{*}\right)-S_{\mathrm{A}} \mathcal{E}_{3}\left(T^{*}\right),
\end{aligned}
$$

donde $\mathcal{D}_{3}\left(T^{*}\right)$ y $\mathcal{E}_{3}\left(T^{*}\right)$ están dadas por 3.42 y 3.43 .

Desde el punto de vista de estados correspondientes, el potencial ANC modificado (3.46), y en específico el segundo coeficiente virial (3.51), se han empleado en sistemas polares (Cap. 4. Sec. 4.4.6) en donde un potencial LJ 12-6 resulta insuficiente para lograr una conformalidad requerida para este tipo de fluidos. 


\section{Referencias}

[1] A. Gil-Villegas and F. del Río. Phys. Rev. E, 53(3):2326-2336, 1996.

[2] F. del Río, J. E. Ramos, A. Gil-Villegas, and I. A. McLure. J. Phys. Chem., 100:9104-9115, 1996.

[3] F. del Río, J. E. Ramos, and I. A. McLure. J. Phys. Chem. B, 102:10568-10575, 1998.

[4] J. E. Ramos, F. del Río, and I. A. McLure. J. Phys. Chem. B, 102:10576-10585, 1998.

[5] I. A. McLure, J. E. Ramos, and F. del Río. J. Phys. Chem. B, 103:7019-7030, 1999.

[6] J. H. Dymond, K. N. Marsh, R. C. Wilhoit, and K. C. Wong. Virial Coefficients of Pure Gases and Mixtures, in: Landolt-Börnstein, Numerical Data and Functional Relationships in Science and Technology, in: M. Frenkel, K.N. Marsh (Eds.), New Series, Group IV: Physical Chemistry, volume 21. Springer-Verlag, 2002 .

[7] B. Ibarra-Tandi and F. del Río. Phys. A, 388:4315-4327, 2009.

[8] I. G. Kaplan. Intermolecular Interactions: Physical Picture, Computational Methods and Model Potentials. John Wiley and Sons,Ltd, 2006.

[9] O. Guzman and F. del Río. J. Phys. B: At. Mol. Opt. Phys., 40:3989-4003, 2007.

[10] O. Guzman and F. del Río. J. Phys. Chem. B, 105:8220-8229, 2001.

[11] F. del Río, I. A. McLure, J. Chávez, J. E. Ramos, and E. Ávalos. Mol. Phys., 104(22-24):3757-3775, 2006.

[12] F. del Río and O. Guzmán. Mol. Phys., 110(11-12):1261-1267, 2012.

[13] G. C. Maitland, M. Rigby, E. B. Smith, and W. A. Wakeham. Statistical Intermolecular Forces. Clarendon Press, Oxford, 1981.

[14] F. del Río, J. E. Ramos, and I. A. McLure. Phys. Chem. Chem. Phys., 1:4937-4945, 1999.

[15] J. E. Ramos, F. del Río, and I. A. McLure. Phys. Chem. Chem. Phys., 3:2634-2643, 2001.

[16] J. A. González-Calderón and F. del Río. Collect. Czech. Chem. Commun., 74(2):363-391, 2009.

[17] J. E. Ramos, F. del Río, and I. A. McLure. Phys. Chem. Chem. Phys., 2:2731-2741, 2000.

[18] F. del Río, E. Díaz-Herrera, and E. Ávalos. J. Chem. Phys., 122:034504, 2005.

[19] O. Guzmán, F. del Río, and J. E. Ramos. Mol. Phys., 109(6):955-967, 2011.

[20] F. del Río, O. Guzman, J. E. Ramos, and B. Ibarra-Tandi. Fluid Ph. Eq., 259:9-22, 2007.

[21] J. E. Ramos, F. del Río, and A. F. Estrada-Alexanders. Int. J. Thermophys., 20(2), 1999.

[22] F. del Río, B. Ibarra-Tandi, and L. Mier y Terán. Mol. Phys., 101(19):2997-3007, 2003.

[23] J. E. Ramos. Mol. Phys., 103(17):2323-2334, 2005.

[24] A. J. M. Garrett. J. Phys. A: Math. Gen., 13:379-380, 1980.

[25] W. Witschel. Int. J. Thermophys., 11(6):1075-1085, 1990.

[26] T. Kihara. J. Phys. Soc. Japan, 6(5):289-296, 1951. 
[27] K Yamazaki and T. Kihara. J. Stat. Phys., 14(6):509-519, 1976.

[28] J. A. González-Calderón. Módelos esféricos y lineales del potencial de moléculas pequeñas no polares. Ph. D. Thesis. Universidad Autónoma Metropolitana., México, 2011.

[29] I. S. Gradshteyn and I. M. Ryzhik. Table of Integrals, series, and products. Elsevier, 2007.

[30] M. Abramowitz and I. A. Stegun. Handbook of Mathematical Functions with Formulas, Graphs, and Mathematical Tables. Dover, New York, 1972. 


\section{Capítulo 4}

\section{Sistemas polares}

\subsection{Introducción}

El carácter eléctrico inherente en las moléculas es el principal factor que define las propiedades fisicoquímicas de la materia; es decir, la interacción entre las distribuciones de carga existentes en las moléculas produce las distintas fases de la materia. En específico, las propiedades termodinámicas de los sistemas polares han sido ampliamente investigadas mediante distintas técnicas tanto teóricas [1,2] como por simulación por computadora [3]. En el estudio de estos sistemas, una gran cantidad de información para fluidos polares se ha obtenido empleando el modelo molecular Stockmayer (SM) el cual consiste de una interacción tipo LJ más la interacción existente entre dos dipolos permanentes puntuales [4]. Cuando se requiere estudiar efectos de polarización regularmente se suman al potencial SM términos extras que simulan la inducción electrostática entre las moléculas [5].

Aunque esos potenciales sean una idealización de las interacciones entre moléculas polares reales, han sido aplicados de manera exitosa para describir las propiedades de fluidos puros y sus mezclas; sin embargo, tanto su dependencia angular como los efectos electrostáticos de largo alcance los hacen poco manipulables tanto a nivel teórico como computacional. Uno de los objetivos de la presente investigación consiste en hallar un potencial simple y esférico que reproduzca la termodinámica de un sistema polar para fases fluidas y que además permita entender los efectos electrostáticos de la frustración a nivel molecular.

Para lo anterior se estudia la conformalidad entre el potencial SM polarizable (SMP) y el efectivo, lo que permite aprovechar el principio de estados correspondientes (PEC); así, si ambas funciones son conformales en un estado dado dicho principio indica que las propiedades termodinámicas del sistema SM se pueden obtener a partir de las del efectivo. Los sistemas efectivos tomados para este estudio son los fluidos LJ 12-6 y ANC 2-S aprovechando las ventajas que cada caso aporta: en el primero de ellos se aprovecha el conocimiento completo de su termodinámica y con el segundo se tiene un mejor control de la conformalidad entre sistemas. Diversos autores han trabajado con sistemas polares efectivos, principalmente a bajas densidades [6-9], que emplean una función SM, y aproximaciones de la misma; muchos de esos resultados corresponden a casos especiales dentro del modelo efectivo actual.

\subsection{Antecedentes}

Los primeros estudios de sistemas polares desde el punto de vista ANC utilizan al potencial SM, ec. (2.16] [10]. El segundo coeficiente virial es obtenido para intensidades pequeñas del dipolo y los resultados son compatibles con los obtenidos por otros autores por debajo de la temperatura de inversión; es decir, aquella donde el segundo coeficiente virial tiene su máximo. 
La inversión de datos teóricos de dicho coeficiente mediante el modelo ANC, ecs. (3.23) y (3.24, arroja parámetros efectivos de distancia y energía acorde al hecho de que se le suma al potencial LJ un término atractivo: el tamaño efectivo de la molécula disminuye y la profundidad del potencial efectivo crece a medida que aumenta la intensidad del dipolo. En cuanto a la suavidad, tanto repulsiva como atractiva, en promedio disminuye a medida que la intensidad del dipolo aumenta ${ }^{1}$

La aplicación de este modelo, utilizando parámetros moleculares constantes, a gases reales se ha hecho inicialmente con aquellas sustancias compuestas por moléculas heterodiatómicas y poliatómicas (y sus mezclas con gases nobles), ambos tipos de moléculas caracterizados por tener dipolos permanentes pequeños [10], muestran resultados acordes a los experimentales.

El tema de los gases polares usando la visión ANC es retomado posteriormente por Ávalos et. al. [11] empleando como principal herramienta el principio de estados correspondientes, y permitiendo que los parámetros efectivos dependan del estado del sistema. Aunque existen estudios similares a éste [7], la inclusión del parámetro suavidad agrega versatilidad al trabajo y permite extender el concepto para momentos dipolares y densidades mayores, haciendo posible encontrar propiedades termodinámicas a nivel del líquido SM y su aplicación para el equilibrio líquido vapor del $\mathrm{HCl}$.

\subsection{Fluidos polares}

Una molécula polar es aquella caracterizada por un momento dipolar eléctricd ${ }^{2}$ permanente, el cual se origina por diferencia de electronegatividad de los átomos que conforman a la molécula. La distribución de carga de un sistema molecular está básicamente determinada por las cargas nucleares localizadas en las distribuciones de densidad nuclear y electrónica alrededor del núcleo. Tanto las moléculas no polares como las polares pueden adquirir un momento dipolar inducido bajo la acción de un campo eléctrico externo que resulta de un experimento o del ambiente molecular de una molécula; este efecto inductivo es temporal y desaparece cuando también lo hace el campo externo. A esta redistribución de cargas debida a la inducción se le conoce como polarización y el término molecular que la mide es la polarizabilidad denotada por la letra griega $\alpha$. Al conjunto completo de interacciones (permanentes o inducidas) entre dipolos se le dará el nombre de interacción dipolo-dipolo.

\subsubsection{Multipolos eléctricos}

En teoría electrostática una distribución de carga $\rho\left(\mathbf{r}^{\prime}\right)$ de una parte restringida del espacio, puede caracterizarse en términos de sus momentos multipolares. Éstos son los coeficientes de un desarrollo en serie del potencial de la distribución de carga $\phi(\mathbf{r})$, en un punto $\mathbf{r}$ debido a toda esa distribución. De acuerdo a la teoría electrostática, el potencial $\phi(\mathbf{r})$ está dado por la ley de Coulomb como

$$
\phi(\mathbf{r})=\frac{1}{4 \pi \epsilon_{0}} \int \frac{\rho(\mathbf{r})}{\left|\mathbf{r}-\mathbf{r}^{\prime}\right|} d \mathbf{r}^{\prime}
$$

\footnotetext{
${ }^{1}$ Este resultado, como se verá posteriormente, se contrapone con la inclusión del resto de las configuraciones omitidas.

${ }^{2}$ Un dipolo eléctrico simple está formado por dos cargas eléctricas $q$ y $-q$ separadas una distancia $R$. Esta distribución de cargas se representa mediante el vector momento dipolar eléctrico $\boldsymbol{\mu}$ dirigido desde la carga negativa a la positiva. El módulo del vector dipolo es $q R$ y regularmente se utilizan unidades de debyes (D) para medirlo,
}

$$
1 \mathrm{D}=3.33564 \times 10^{-30} \mathrm{C} \mathrm{m} \text {. }
$$


donde $\epsilon_{0}$ es la permitividad del vacío. Suponiendo que $|\mathbf{r}| \gg\left|\mathbf{r}^{\prime}\right|$, se tiene que

$$
\begin{aligned}
\left|\mathbf{r}-\mathbf{r}^{\prime}\right|^{-1} & =r^{-1}\left[1-\frac{2 \mathbf{r} \cdot \mathbf{r}^{\prime}}{r^{2}}+\left(\frac{r^{\prime}}{r}\right)^{2}\right]^{-1 / 2} \\
& =r^{-1}\left[1-\frac{\mathbf{r} \cdot \mathbf{r}^{\prime}}{r^{2}}-\frac{1}{2}\left(\frac{r^{\prime}}{r}\right)^{2}+\frac{3}{2} \frac{(\mathbf{r} \cdot \mathbf{r})^{2}}{r^{4}}+\ldots\right] .
\end{aligned}
$$

Combinando los términos subsecuentes en la ec. (4.2) de acuerdo a sus potencias en $\left(r^{\prime} / r\right)^{n}$ con la ec. (4.1) se forma el desarrollo multipolar del potencial $\phi\left(\mathbf{r}^{\prime}\right)[12]$.

En específico, el término dipolar es

$$
\begin{aligned}
\phi^{(2)}(\mathbf{r}) & =\frac{1}{4 \pi \epsilon_{0}} \frac{\mathbf{r}}{r^{3}} \cdot \int \mathbf{r}^{\prime} \rho\left(\mathbf{r}^{\prime}\right) d r^{\prime}=\frac{1}{4 \pi \epsilon_{0}} \frac{\boldsymbol{\mu} \cdot \mathbf{r}}{r^{3}} \\
& =\frac{1}{4 \pi \epsilon_{0}} \frac{\mu_{\alpha}}{r^{2}} \frac{r_{\alpha}}{r}
\end{aligned}
$$

donde el momento dipolar (vector) de la distribución de carga (un tensor de primer rango) es denotado por $\boldsymbol{\mu}$ y donde las tres componentes del tensor momento dipolar son

$$
\mu_{\beta}=\int r_{\beta}^{\prime} \rho\left(\mathbf{r}^{\prime}\right) d \mathbf{r}^{\prime}
$$

\subsubsection{Potencial de interacción entre dos dipolos}

Habiendo expresado el potencial $\phi(\mathbf{r})$ como un desarrollo en serie de $1 / r$, el desarrollo correspondiente para el campo eléctrico $\mathbf{E}(\mathbf{r})$ puede obtenerse por diferenciación

$$
\mathbf{E}(\mathbf{r})=-\nabla \phi(\mathbf{r})
$$

que para el término dipolar es 3

$$
\begin{aligned}
\mathbf{E}^{(2)}(\mathbf{r}) & =\frac{1}{4 \pi \epsilon_{0}}\left[\frac{3 \mathbf{r}(\boldsymbol{\mu} \cdot \mathbf{r})}{r^{5}}-\frac{\boldsymbol{\mu}}{r^{3}}\right] \\
& =\frac{1}{4 \pi \epsilon_{0}} \frac{\boldsymbol{\mu}}{r^{3}} \cdot\left[\frac{3 \mathbf{r r}}{r^{2}}-1\right],
\end{aligned}
$$

obteniéndose un potencial de interacción dipolo-dipolo $(d d)$

$$
\begin{aligned}
\Phi_{i j}^{d d} & =\boldsymbol{\mu}_{j} \cdot \mathbf{E}_{i}^{(2)}(\mathbf{r})=\frac{1}{r^{3}}\left[\boldsymbol{\mu}_{i} \cdot \boldsymbol{\mu}_{j}-3\left(\boldsymbol{\mu}_{i} \cdot \hat{\mathbf{r}}\right)\left(\boldsymbol{\mu}_{j} \cdot \hat{\mathbf{r}}\right)\right] \\
& =-\frac{\mu^{2}}{r^{3}}\left[2 \cos \theta_{i} \cos \theta_{j}-\sin \theta_{i} \sin \theta_{j} \cos \left(\phi_{i}-\phi_{j}\right)\right]
\end{aligned}
$$

para moléculas de una sola especie. El potencial binario Stockmayer (SM), ec. (2.16), proviene precisamente de incrustar dipolos puntuales (interactúando según la expresión anterior) en dos sitios Lennard-Jones 12-6, ec. 2.12. Además de emplearse en sistemas polares simples [1,14], el potencial SM suele usarse en el estudio de polímeros autoensamblados, fluidos ferroeléctricos y magnetoreológicos e inclusive como modelo para cristales líquidos polares [15].

${ }^{3}$ Usando la definición del tensor dipolar [13], el campo originado obtiene la forma

$$
T_{\alpha \beta} \equiv \frac{1}{4 \pi \epsilon_{0}} \frac{1}{r^{3}}\left[\frac{3 r_{\alpha} r_{\beta}}{r^{2}}-\delta_{\alpha \beta}\right],
$$

y las componentes del campo dipolar de convierten en

$$
E_{\alpha}^{(2)}(\mathbf{r})=\frac{1}{4 \pi \epsilon_{0}} \frac{\mu_{\beta}}{r^{3}}\left[\frac{3 r_{\alpha} r_{\beta}}{r^{2}}-\delta_{\alpha \beta}\right]=T_{\alpha \beta} \mu_{\beta}
$$




\subsection{Sistema Stockmayer polarizable}

\subsubsection{Potencial Stockmayer polarizable}

La interacción entre dos moléculas Stockmayer polarizables (SMP), separadas una distancia $r=|\boldsymbol{r}|$, y con orientaciones dadas por $\boldsymbol{\omega}_{i}=\left(\theta_{i}, \phi_{i}\right)$, se define como

$$
\Phi_{\mathrm{SMP}}\left(r, \boldsymbol{\omega}_{i}, \boldsymbol{\omega}_{j}\right)=\Phi_{\mathrm{SM}}\left(r, \boldsymbol{\omega}_{i}, \boldsymbol{\omega}_{j}\right)+\Phi_{i j}^{d i}\left(r, \boldsymbol{\omega}_{i}, \boldsymbol{\omega}_{j}\right)
$$

donde al potencial SM, ecuación (2.16), se le ha sumado la interacción dipolo-dipolo inducido dada por

$$
\Phi_{i j}^{d i}\left(r, \boldsymbol{\omega}_{i}, \boldsymbol{\omega}_{j}\right)=-\frac{\mu^{2} \alpha}{r^{6}} h_{i j}\left(\boldsymbol{\omega}_{i}, \boldsymbol{\omega}_{j}\right)
$$

que incluye una dependencia extra con la polarizabilidad $\alpha$ de la molécula. La función $h_{i j}$ está dada por ${ }^{4}$

$$
h_{i j}=2+3 \cos ^{2} \theta_{i}+3 \cos ^{2} \theta_{j}
$$

cuyos ángulos son presentados en la figura 2.3, ver pág. 12 . Obsérvese que, cuando $\alpha=0$ se regresa a la expresión SM $[17,18]$.

De las expresiones 4.8 y 4.11) es sencillo determinar que la configuración más estable para $\Phi_{\mathrm{SMP}}$ se da cuando los vectores $\boldsymbol{\mu}_{i}$ y $\boldsymbol{\mu}_{j}$ cumplen la configuración cabeza-cola. La configuración menos estable dependerá del sistema que se esté manejando y especialmente de las intensidades del momento dipolar y la polarizabilidad. En el caso SM (cuando no hay polarización entre moléculas) la configuración con mayor valor energético será aquella donde dichos vectores se encuentran (cabeza-cabeza) o se contraponen (cola-cola).

En la figura 4.1 se presenta el perfil del potencial $\Phi_{\text {SMP }}$ para distintos sistemas y configuraciones orientacionales. En la figura principal se muestra el caso en que $\mu^{* 2}=\mu^{2} / \epsilon \sigma^{3}=1.0$ y $\alpha^{*}=\alpha / \sigma^{3}=0.1{ }^{5}$ las curvas a trazos cortos y a trazos largos representan el potencial para las configuraciones más estable e inestable del sistema, respectivamente. La energía de cualquier otra conformación angular posible entre las dos partículas, en el mismo estado, se encontrará entre esos dos casos extremos. En la figura secundaria se presenta la misma situación para $\mu^{* 2}=4.0$. Cotejando ambas figuras se observa el aumento notable (para la configuración más estable, curva a trazos cortos) en la profundidad del pozo de $\Phi_{\mathrm{SMP}}$ cuando la intensidad del momento dipolar se incrementa.

\begin{tabular}{|c|c|c|c|}
\hline$\mu^{* 2}$ & $\frac{\epsilon_{\mathrm{SMP}}^{(0)}-\epsilon_{\mathrm{LJ}}}{\epsilon_{\mathrm{LJ}}}$ & $\frac{\epsilon_{\mathrm{SMP}}^{(1)}-\epsilon_{\mathrm{SMP}}^{(0)}}{\epsilon_{\mathrm{SMP}}^{(0)}}$ & $\frac{\epsilon_{\mathrm{SMP}}^{(2)}-\epsilon_{\mathrm{SMP}}^{(1)}}{\epsilon_{\mathrm{SMP}}^{(1)}}$ \\
$(\%)$ & 52.0 & 36.8 & 2.5 \\
1 & 221.9 & 43.2 & 3.3 \\
2 & 405.2 & 48.5 & 4.0 \\
4 & 600.0 & 53.2 & 4.7 \\
\hline
\end{tabular}

Tabla 4.1: Contribución de los términos electrostáticos al potencial SMP $\left(\alpha^{*}=0.1\right)$ para distintos momentos dipolares, y diferentes órdenes de polarizabilidad.

\footnotetext{
${ }^{4} \mathrm{El}$ término extra surge de considerar un momento inducido sobre el dipolo, generado por un campo eléctrico total homogéneo, dado por $\alpha \mathbf{E}_{i}^{(2)}(\mathbf{r})$ [16].

${ }^{5}$ Aquí se han usado los parámetros LJ, $\epsilon$ y $\sigma$, como unidades de reducción de los parámetros electrostáticos.
} 


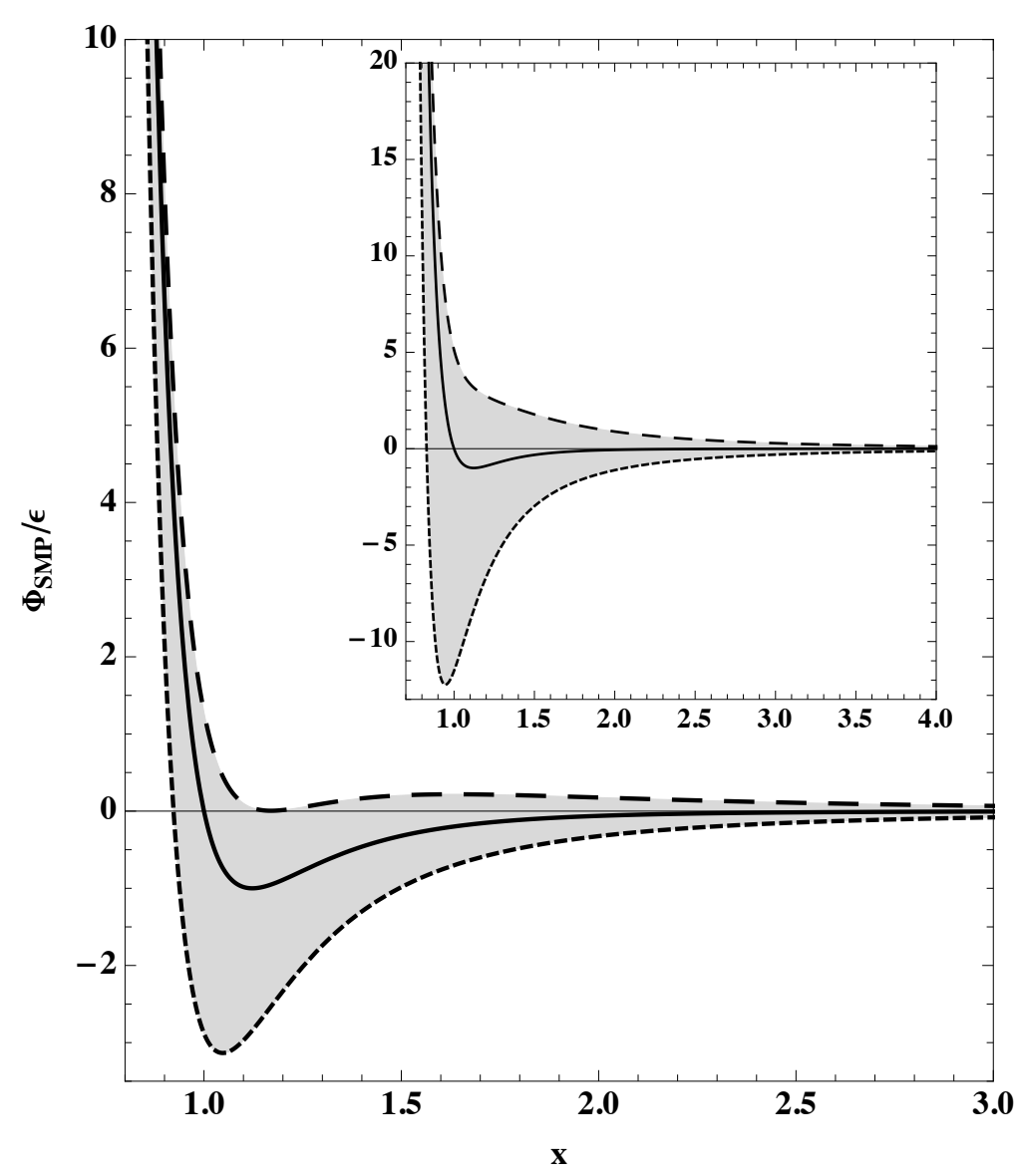

Figura 4.1: Potencial LJ (línea sólida) comparado con el potencial por pares SMP para $\mu^{* 2}=1.0$ y $\alpha^{*}=0.1$. Los centros de las partículas están separados una distancia, $x=r / \sigma$. Dos configuraciones extremas entre dipolos son mostradas: cola-cola (línea a trazos largos) y cabeza-cola (línea a trazos cortos). A una distancia dada, $x$, el potencial SMP puede tomar cualquier valor en la región sombreada dependiendo de la orientación relativa de los dipolos. Una situación equivalente es mostrada en el recuadro con una intensidad dipolar, $\mu^{* 2}=4.0$ y misma polarizabilidad.

En la ecuación (4.9) se han despreciado términos inductivos mayores al de primer orden al considerar que contribuyen poco a dicha energía de interacción. Este hecho se justifica en cierta medida usando la energía del mínimo del potencial. La tabla 4.1 presenta el cambio porcentual de estas energías al incorporar cada término electrostático al potencial LJ según el potencial SM 6 Dichos cálculos se han obtenido considerando la configuración más estable entre los dipolos (cabeza-cola), por lo que para cualquier otro arreglo todas las contribuciones serán menores a las de este caso.

En esta última tabla $\epsilon_{\mathrm{SMP}}^{(i)}$ corresponde al mínimo energético con el $i$-ésimo orden de polarizabilidad. Estos resultados se presentan de manera gráfica en la figura 4.2 donde se muestran los efectos acumulados de cada uno de los términos de $\Phi_{\mathrm{SMP}}$ ocupados en este trabajo.

En la siguiente sección se trabajará con promedios angulares del potencial $\Phi_{\text {SMP }}$ los cuales resultan dependientes de la temperatura. Los perfiles de estos potenciales promediados se hallan en la zona sombreada de la figura 4.1 exactamente entre el perfil LJ (línea sólida) y la configuración más estable (línea a trazos cortos), por lo que realmente la contribución de segundo orden en $\alpha$ será mucho menor a las especificadas en la tabla 4.1. en especial a temperaturas grandes.

\subsubsection{Potencial SMP esfericalizado}

La configuración relativa entre las moléculas que forman un sistema polar dependerá principalmente del campo eléctrico generado por todas ellas y su energía cinética. La primera dependencia tiende a alinear las

\footnotetext{
${ }^{6}$ En específico, el término de segundo orden en la polarizabilidad está dado por

$$
\frac{\mu^{2}}{r^{9}} \alpha^{2}\left[8 \cos \theta_{i} \cos \theta_{j}-\operatorname{sen} \theta_{i} \operatorname{sen} \theta_{j} \cos \left(\phi_{i}-\phi_{j}\right)\right] \text {. }
$$
}




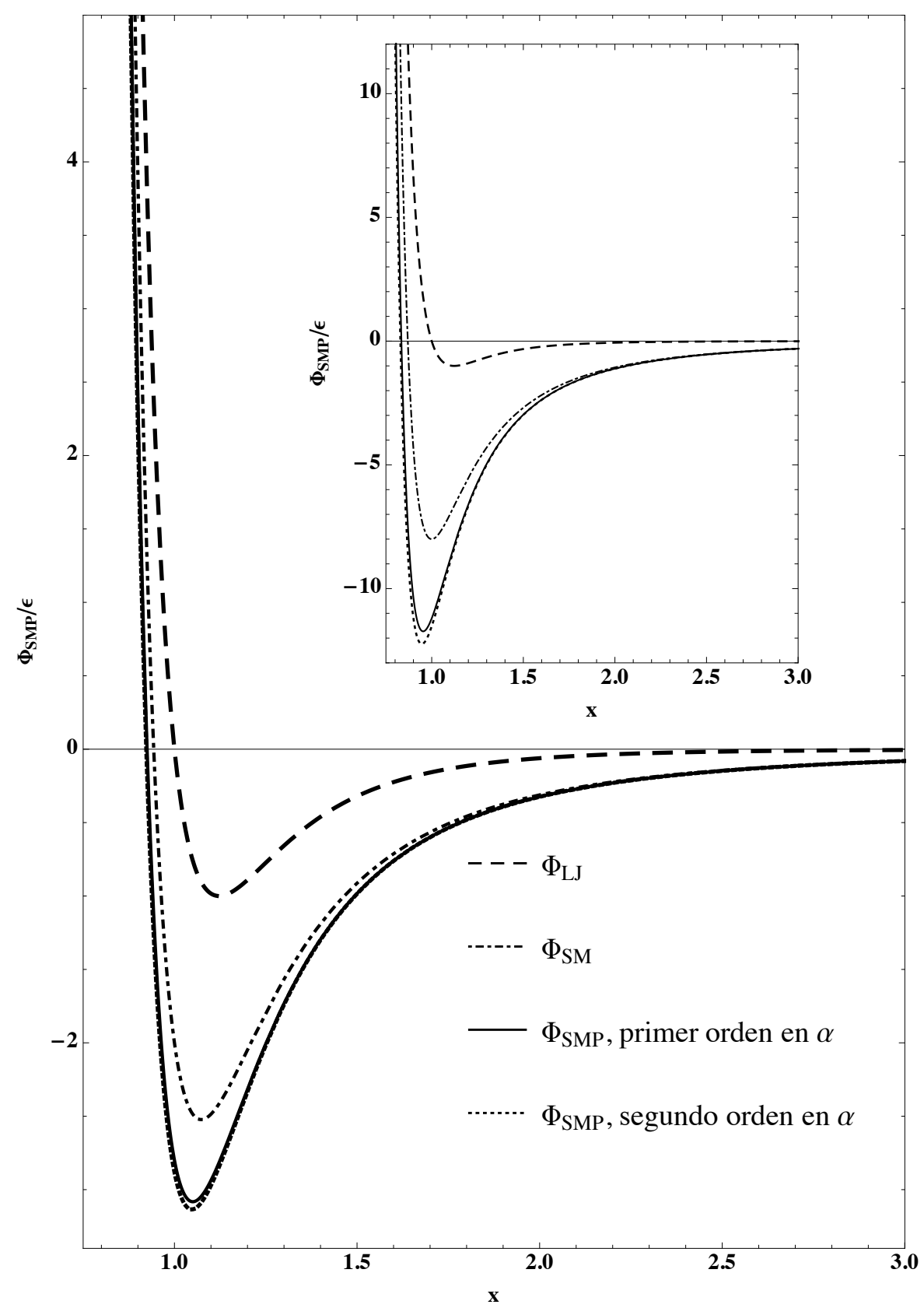

Figura 4.2: Efecto de la polarizabilidad en el potencial $\Phi_{\mathrm{SMP}}$ usando la configuración más estable, cabeza-cola. En la figura principal ( $\mu^{* 2}=1 \mathrm{y} \alpha^{*}=0.1$ ) se observa el cambio al sumar a la función Lennard-Jones el primer y segundo términos de la ecuación (4.17). La línea a trazos cortos muestra el efecto obtenido al agregar el término proporcional a $\alpha^{* 2}$. En el recuadro se muestra la misma situación para el caso $\mu^{* 2}=4$ y la misma polarizabilidad. 
moléculas en un arreglo específico mientras que el segundo factor altera tal disposición de manera aleatoria. Así, cuando la temperatura aumenta, el efecto electrostático entre las moléculas empezará a cobrar menor relevancia. A bajas densidades los dipolos que forman el sistema SMP rotan librement 7 por lo que resulta conveniente cambiar la interacción original $\Phi_{\mathrm{SMP}}$ por una promedio obtenida al considerar todas las configuraciones posibles existentes entre dos dipolos. De esta forma la dependencia angular es eliminada generando una expresión mucho más simple de manipular, simétricamente esférica, y que en esencia contiene la física del sistema SMP. Este proceso de "esfericalización" da como resultado un potencial esférico efectivo (SMPs) que será sustituido en la ec. 2.29 pág. 17 para intentar reproducir alguna propiedad termodinámica $X$ de interés de dicho sistema.8

En específico, si se toma a $X$ como la presión $P$ del sistema, ec. 2.31 pág. 18), se demuestra que a bajas densidades dicha ecuación puede escribirse en términos del segundo coeficiente virial

$$
B_{\mathrm{SMPs}}\left(T,\left[\Phi_{\mathrm{SMP}}\right]\right)=B_{\mathrm{SMP}}\left(T,\left[\Phi_{\mathrm{SMP}}\right]\right)
$$

donde el coeficiente del lado derecho corresponde al potencial original $\Phi_{\mathrm{SMP}}$ mientras que el del lado izquierdo es el coeficiente virial de la interacción efectiva SMPs.

Consideremos los dos coeficientes por separado; por un lado, el segundo coeficiente virial para un fluido SMP, ec. 4.9, está dado por

$$
B_{\mathrm{SMP}}(T)=\frac{1}{4} \int d \Omega \int_{0}^{\infty} d r\left[1-\exp \left(-\frac{\Phi_{\mathrm{SMP}}}{k_{\mathrm{B}} T}\right)\right] r^{2}
$$

donde $\Omega$ representa el conjunto total de ángulos que definen la orientación entre las dos moléculas SMP y cuya notación permite escribir la diferencial angular como $d \Omega \equiv \operatorname{sen} \theta_{i} d \theta_{i} \operatorname{sen} \theta_{j} d \theta_{j} d\left(\phi_{i}-\phi_{j}\right)$.

Por otro lado, el potencial esfericalizado $\Phi_{\mathrm{SMPs}}$ tiene, por definición, un segundo coeficiente virial de la forma

$$
B_{\mathrm{SMPs}}(T)=2 \pi \int_{0}^{\infty} d r\left[1-\exp \left(-\frac{\Phi_{\mathrm{SMPs}}}{k_{\mathrm{B}} T}\right)\right] r^{2}
$$

donde el factor $2 \pi$ surge debido a que no existe dependencia angular en $\Phi_{\mathrm{SMPs}}$.

De acuerdo a la ecuación 4.12, los coeficientes 4.13) y 4.14 son iguales, por lo que después de unos pasos algebraicos se tiene que el potencial esfericalizado tiene la forma

$$
\Phi_{\mathrm{SMP}}(r)=-k_{\mathrm{B}} T \ln \left\{\frac{\int d \Omega \exp \left(-\frac{\Phi_{\mathrm{SMP}}}{k_{\mathrm{B}} T}\right)}{\int d \Omega}\right\}
$$

donde $\int d \Omega=8 \pi$. La ecuación 4.15 expresa de forma explícita al potencial esfericalizado para un fluido SMP y debido a que el factor de Boltzmann se encuentra involucrado surge una dependencia natural en la temperatura.

Debido a que el potencial SMP contiene un kernel esférico del tipo LJ, la ecuación 4.15 puede reescribirse como

$$
\Phi_{\mathrm{SMPs}}(r)=\Phi_{\mathrm{LJ}}(r)-k_{\mathrm{B}} T \ln \left\{\frac{\int d \Omega \exp \left(-\frac{\Phi_{\mathrm{el}}}{k_{\mathrm{B}} T}\right)}{\int d \Omega}\right\}
$$

donde $\Phi_{e l}$ es la contribución electrostática dada por

$$
\Phi_{e l}=-\frac{\mu^{2}}{r^{3}} g_{i j}\left(\boldsymbol{\omega}_{i}, \boldsymbol{\omega}_{j}\right)-\frac{\mu^{2} \alpha}{r^{6}} h_{i j}\left(\boldsymbol{\omega}_{i}, \boldsymbol{\omega}_{j}\right)
$$

que resulta de sumar $\Phi_{i j}^{d d}$ dada por la ec. 4.8 y el segundo término de la ec. 4.9.

\footnotetext{
${ }^{7}$ Esta condición es válida para grandes separaciones o en un medio con una constante dieléctrica $(\varepsilon)$ alta, cuando la energía de interacción, dependiente de la orientación, está por debajo de la energía térmica $k_{\mathrm{B}} T$ [19].

${ }^{8} \mathrm{El}$ proceso de esfericalización es un caso especial del teorema de distribución de potencial usado para obtener la energía libre de Helmholtz de una energía libre dependiente de las orientaciones [19].
} 
La forma matemática para este potencial nos conduce a un término atractivo respecto al potencial LJ lo que indica que todas las curvas de los potenciales SMPs estarán (gráficamente) por debajo de dicha función y por arriba de aquella cuya configuración es la más estable (zonas grises de la figura 4.1).

La estructura que presenta la ecuación 4.15 recuerda a la energía libre de Helmholtz $A$ escrita en el ensamble canónico [20]; en este caso, entre dos dipolos que rotan libremente. En efecto, derivando la ecuación (4.15) respecto a la temperatura se llega a la expresión

$$
\Phi_{\mathrm{SMPs}}=\Phi_{\mathrm{SMP}}+T \frac{d \Phi_{\mathrm{SMPs}}}{d T}
$$

por lo que la energía libre de Helmholtz $A(=U-T S)$ se identifica con la función $\Phi_{\mathrm{SMPs}}$ y a $\Phi_{\mathrm{SMP}}$ con la energía interna dada por $1^{9}$

$$
\Phi_{\mathrm{SMP}}=\frac{\int d \Omega \Phi_{\mathrm{SMP}} \mathrm{e}^{-\Phi_{\mathrm{SMP}} / k_{\mathrm{B}} T}}{\int d \Omega \mathrm{e}^{-\Phi_{\mathrm{SMP}} / k_{\mathrm{B}} T}}
$$

Es decir, el potencial $\Phi_{\mathrm{SMPs}}$ está formado por dos términos independientes: uno energético y uno relacionado con la entropía orientacional entre dos dipolos [9]. En otras palabras, la función entre los corchetes de 4.15] es la función de partición canónica del sistema SMP y el potencial resultante es precisamente el promedio energético de todas las configuraciones posibles entre dos moléculas regidas por la interacción $\Phi_{\mathrm{SMP}}$.

\subsubsection{Desarrollo numérico del potencial esfericalizado}

Aunque la integral principal localizada en la ecuación (4.16) puede resolverse numéricamente se ha optado por tomar una ruta alterna, empleada por diversos autores, en la cual se desarrolla el factor de Boltzmann y posteriormente se integra el término general de dicho desarrollo. Para realizar este proceso se han definido cantidades reducidas usando los parámetros $\sigma$ y $\epsilon$ del kernel esférico como unidades de reducción. Dichas cantidades son presentadas en la tabla 4.2 .

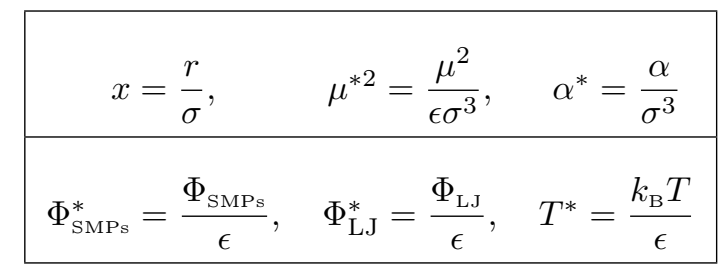

Tabla 4.2: Reducción de cantidades electrostáticas para el modelo SMP.

Con esta cantidades el potencial SMP esfericalizado reducido tiene la forma

$$
\Phi_{\mathrm{SMPs}}^{*}(x)=\Phi_{\mathrm{LJ}}^{*}(x)-T^{*} \ln \left[\sum_{m, n=0}^{\infty} \frac{\left\langle g_{i j}^{m} h_{i j}^{n}\right\rangle_{\Omega}}{m ! n !}\left(\frac{\mu^{* 2}}{T^{*} x^{3}}\right)^{m+n}\left(\frac{\alpha^{*}}{x^{3}}\right)^{n}\right]
$$

donde el término identificado por los corchetes $\langle\cdots\rangle$ es la integral

$$
\left\langle g_{i j}^{m} h_{i j}^{n}\right\rangle_{\Omega}=\frac{1}{8 \pi} \int d \Omega g_{i j}^{m} h_{i j}^{n}
$$

cuya solución puede aproximarse a la forma

$$
\left\langle g_{i j}^{m} h_{i j}^{n}\right\rangle_{\Omega}=\sum_{k=0}^{m} \sum_{l=0}^{n} \sum_{q=0}^{l}\left(\begin{array}{c}
m \\
k
\end{array}\right)\left(\begin{array}{l}
n \\
l
\end{array}\right)\left(\begin{array}{l}
l \\
q
\end{array}\right) \frac{\mathrm{B}\left(\frac{k+1}{2}, \frac{1}{2}\right) \mathrm{B}\left(\frac{k+2}{2}, \frac{m-k+2 q+1}{2}\right) \mathrm{B}\left(\frac{k+2}{2}, \frac{m-k+2 l-2 q+1}{2}\right)}{2^{2-(m-k)-(n-l)} 3^{-l} \pi}
$$

\footnotetext{
${ }^{9} \mathrm{El}$ denominador en esta expresión forma parte de un grupo conocido como integrales de Keesom [17,21,22], que en el caso de sustancias polares, sin el carácter inductivo, pueden simplificarse pasando de una integral múltiple a una simple mucho más sencilla de resolver numéricamente [23].
} 
escrita en términos de funciones beta, $\mathrm{B}(x, y)$ [24]. Rutinas de dicha expresión son sencillas de implementar en cualquier software matemático.

En general, no se requiere gran esfuerzo computacional para realizar cálculos con la expresión 4.20, y normalmente la convergencia se logra con pocos términos; sin embargo, la combinación: $x$ pequeñas, $T^{*}$ bajas y/o $\mu^{*}$ grandes, resulta en una convergencia muy lenta de esta suma. Y ya que se manejarán varios estados del sistema SMPs resulta conveniente encontrar un número mínimo de términos que agilicen estos cálculos.

En principio, nos hemos concentrado en sistemas SMPs en los estados: $T^{*} \in[0.3,10.0], \mu^{* 2} \in[0.0,4.0]$ y $\alpha^{*} \in[0.0,0.1]$, por lo que los valores $T^{*}=0.3, \mu^{* 2}=4.0$ y $\alpha^{*}=0.1$, definirán los números $m$ y $n$ requeridos para 4.20); en cualquier otro estado se requerirá un número menor de términos. Además, como se argumenta en la sección 4.4.5, al usar el PEC sólo se emplearán aquellos parámetros que identifican al mínimo de un potencial SMPs, por lo tanto no es necesario considerar valores pequeños para $x$, sino del orden de la unidad, —obsérvese la figura $4.3(\mathrm{a})$ - Especificado lo anterior, se establece que con valores $m=30$ y $n=10$ usados en la suma 4.20 se obtienen parámetros $\epsilon_{\text {ef }}$ y $\delta_{\text {ef }}$ con un error relativo al $1 \%$.

Los primeros términos de la ecuación 4.20 (cuando $n=0$ ) han sido utilizados en diversos estudios con fluidos SM. Usando el primer término distinto de cero en $m$ se obtiene la mitad del potencial de Keesom[11][6], utilizado regularmente en este tipo de sistemas [8,26-28]. Los primeros dos términos hasta un orden $x^{-12}$ fueron incluidos por Cook et. al. [7] en su aplicación del PEC a sistemas SM y posteriormente por otros investigadores [29-32]. En lo que se refiere a sistemas polarizables no se tiene conocimiento alguno de desarrollos, en esta misma dirección, aplicados a potenciales esfericalizados.

\subsubsection{Una forma analítica para el potencial SMP}

Como una primera aproximación pueden retenerse tan solo los primeros términos de (4.20) hasta el orden $x^{-12}$ y primer orden en $\alpha^{*}$ obteniéndose un potencial efectivo de la forma

$$
\Phi_{\mathrm{ef}}^{*}=\left(4-\frac{2 \alpha^{*} \mu^{* 6}}{15 T^{* 2}}+\frac{7 \mu^{* 8}}{450 T^{* 3}}\right) \frac{1}{x^{12}}-\left(4+2 \alpha^{*} \mu^{* 2}+\frac{\mu^{* 4}}{3 T^{*}}\right) \frac{1}{x^{6}}
$$

y, además considerando que tiene la misma forma que el potencial LJ 12-6, se procede de la misma manera que Cook et. al. [7] y Avalos et al. [11] se obtienen parámetros efectivos de energía y distancia dados por

$$
\begin{aligned}
\frac{\epsilon_{\mathrm{ef}}}{\epsilon}=1+\frac{\mu^{* 4}}{6 T^{*}}+\left(1-\frac{14}{25 T^{*}}\right) \frac{\mu^{* 8}}{144 T^{* 2}} & +\left[1+\frac{\mu^{* 4}}{12 T^{*}}\left(1+\frac{2}{5 T^{*}}\right)\right] \mu^{* 2} \alpha^{*} \\
\frac{\delta_{\mathrm{ef}}}{\sigma}=2^{1 / 6}\left\{1-\frac{\mu^{* 4}}{72 T^{*}}+\right. & \left(1-\frac{24}{25 T^{*}}\right) \frac{7 \mu^{* 8}}{10368 T^{*}} \\
- & \left.\frac{1}{12}\left[1-\frac{7}{72} \frac{\mu^{* 4}}{T^{*}}\left(1-\frac{24}{35 T^{*}}\right)\right] \mu^{* 2} \alpha^{*}\right\},
\end{aligned}
$$

que son la distancia y energía reducidos del mínimo del potencial 4.22. Cuando en estas dos expresiones se considera polarizabilidad nula se recuperan los resultados presentados en las dos referencias antes citadas. En pocas palabras, el potencial 4.22 puede ser escrito como un potencial LJ con parámetros moleculares 4.23) y 4.24, los cuales dependen del estado del sistema.

El proceso anterior muestra que al realizar la equivalencia entre el potencial trunco 4.22 y un potencial LJ se generan parámetros efectivos 4.23 y 4.24 los cuales ubican el mínimo del primero de los potenciales.

\footnotetext{
${ }^{10}$ Sólo deben considerarse los términos pares para $k, m$ y $m-k$, haciéndose nulos aquellos valores para valores impares.

${ }^{11}$ En realidad Keesom trabajó erróneamente con la energía interna del sistema dipolo-dipolo en lugar de hacerlo con la energía libre [25].
} 
Esta misma idea será empleada posteriormente de una manera más extensa considerando el desarrollo completo del potencial esfericalizado 4.20). Así, primero se ubica el mínimo del esfericalizado y con él se intenta obtener alguna equivalencia con un potencial mucho más sencillo. A partir de esos resultados el comportamiento de los parámetros efectivos podrá compararse con los ahora obtenidos.

\subsubsection{Conformalidad entre potenciales SMP y LJ}

Como ya se había mencionado, y como puede verse a partir de la ecuación 4.20 , el efecto neto de esfericalizar el potencial $\Phi_{\mathrm{SMP}}^{*}$ resulta en un potencial más atractivo que el LJ a medida que las propiedades electrostáticas del sistema crecen en intensidad. Dicho efecto es apreciado en la figura 4.3(a) en ella se muestra el comportamiento para un conjunto de potenciales $\Phi_{\mathrm{SMPs}}^{*}$ para distintas intensidades del momento dipolar manteniendo constantes $T^{*}$ y $\alpha^{*}$. Claramente se observa cómo el mínimo del potencial $\Phi_{\mathrm{SMPs}}^{*}$ se hace más profundo y se desplaza hacia la izquierda mientras $\mu^{*}$ aumenta. Un efecto semejante se observa cuando la temperatura disminuye.

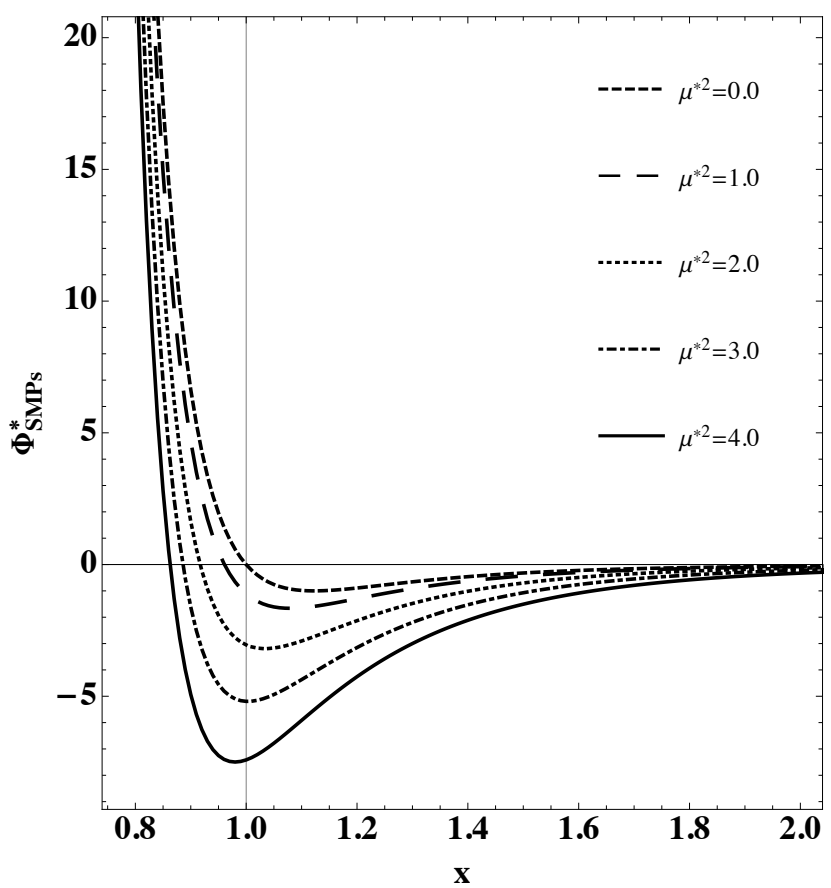

(a)

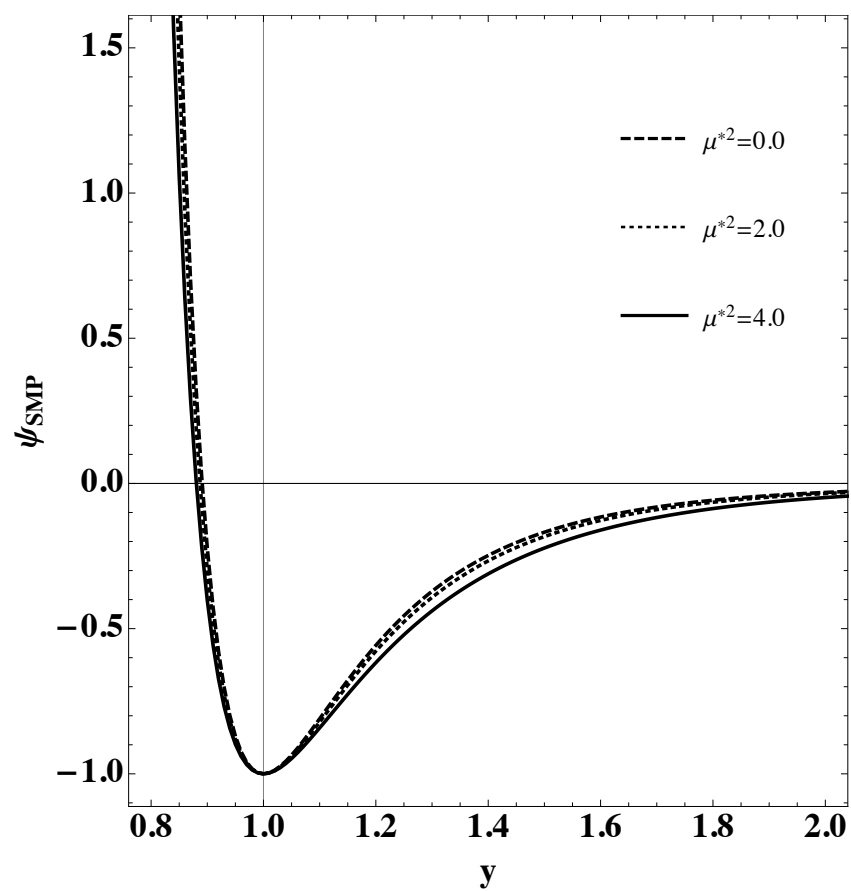

(b)

Figura 4.3: (a) Dependencia con $\mu^{*}$ del potencial $\Phi_{\mathrm{SMPs}}^{*}$. Potencial SMPs para distintos momentos dipolares, todas a $T^{*}=0.3$ y $\alpha^{*}=0.1$. (b) Potencial esfericalizado re-escalado $\psi_{\mathrm{SMP}}$. Las funciones $\Phi_{\mathrm{SMPs}}^{*}$ de la figura contigua son reescaladas para comparar sus perfiles con la función Lennard-Jones. Para momentos dipolares menores a 1.0 las gráficas se superponen con la de la función LJ (curva superior).

Para un sistema dado, el potencial $\Phi_{\mathrm{SMPs}}$ tiene un mínimo caracterizado por la distancia, $\delta_{\mathrm{ef}}$, y energía, $\epsilon_{\mathrm{ef}}$; esto significa, $\Phi_{\mathrm{SMPs}}\left(\delta_{\mathrm{ef}}\right)=-\epsilon_{\mathrm{ef}}$. Usando a $\epsilon$ y $\sigma$ del potencial LJ como parámetros de reducción se establecen las siguientes cantidades

$$
\begin{aligned}
& F_{\epsilon}=\frac{\epsilon_{\mathrm{ef}}}{\epsilon} \\
& F_{\delta}=\frac{\delta_{\mathrm{ef}}}{\sigma}
\end{aligned}
$$


con los cuales se reescala el potencial $\Phi_{\mathrm{SMPs}}^{*}$, para definir una nueva versión del potencial esfericalizado

$$
\psi_{\mathrm{SMP}}(y)=\frac{\Phi_{\mathrm{SMPs}}^{*}\left(y=x / F_{\delta}\right)}{F_{\epsilon}},
$$

que cumple la relación $\psi_{\mathrm{SMP}}(y=1)=-1$. Visualmente, el perfil $\psi$ de cualquier sistema puede compararse de manera directa con el respectivo reescalamiento del potencial LJ $\left(\psi_{\mathrm{LJ}}\right)$, estableciendo así una técnica semiempírica para identificar conformalidad entre el sistema de estudio y el sistema Lennard-Jones. Un resultado importante puede obtenerse tomando el conjunto de potenciales $\Phi_{\text {SMPs }}^{*}$ presentes en la figura 4.3(a) y a continuación aplicarles el reescalamiento respectivo. El nuevo conjunto de potenciales $\psi$, junto con el potencial $\psi_{\mathrm{LJ}}$, se muestran en la figura $4.3(\mathrm{~b})$.

Tomando en cuenta la visión establecida por el PEC y lo expresado en la parte final de la sección anterior, se reemplazará la interacción esfericalizada $\Phi_{\mathrm{SMPs}}^{*}$ por una función LJ con parámetros efectivos. Como ya se ha visto en la sección 2.4 el PEC establece que si una familia de potenciales del mismo tipo puede ser superpuesta, toda ella en una función potencial única, esta última caracterizará a toda la familia desde el punto de vista termodinámico.

De la figura 4.3 se desprenden los siguientes hechos: para el intervalo de temperaturas aquí manejado, los sistemas con $\mu^{* 2} \lesssim 1.0$ son prácticamente conformales a un sistema LJ (aún para polarizabilidades altas); es decir, para estos sistemas se espera pueda aplicarse el PEC. Cuando la intensidad dipolar aumenta y la temperatura disminuye dicho principio dejará de cumplirse lo que implica que los perfiles de $\psi_{\mathrm{SMP}}$ empiezan a no coincidir con la función LJ.

De esta forma cualquier otro potencial $\psi_{\mathrm{SMP}}$, con intensidades dipolares menores a los usados en este ejemplo, quedarán entre las curvas superior e inferior de la figura 4.3(b). De forma similar se puede mostrar que para $T^{*} \gtrsim 1.0$ cualquier sistema con $\mu^{* 2} \leq 4.0$ y $\alpha^{*} \leq 0.1$ son aproximadamente conformales a LJ (línea sólida en la figura). Para el resto de potenciales $\psi_{\mathrm{SMP}}$ no conformales (ejemplificados por las curvas que no se superponen en la figura 4.3(b) se considerarán aproximadamente conformales a una función LJ.

Dos puntos importantes pueden extraerse de lo anterior: i) la construcción de $\psi$ tiene como única finalidad la de mostrar conformalidad entre el sistema Lennard-Jones y nuestro sistema de interés $\Phi_{\mathrm{SMPs}}^{*}$, y ii) verificado lo anterior, las sustituciones $\epsilon \rightarrow \epsilon_{\mathrm{ef}} \mathrm{y} \delta \rightarrow \delta_{\mathrm{ef}}$ en la función Lennard-Jones generará un potencial efectivo del sistema SMPs, de acuerdo al principio de estados correspondientes.

\subsubsection{Teoría ANC para un fluido SMP}

Una vía alterna para verificar de manera cuantitativa la conformalidad entre dos potenciales es mediante la teoría ANC. En el caso de sistemas SMP la teoría ANC, en su forma modificada - ver sección 3.5.4 -, puede introducirse relacionando a los potenciales $\Phi_{\mathrm{ANC}}^{\prime}$ y $\Phi_{\mathrm{SMPs}}$. El hecho de que el potencial ANC modificado $\Phi_{\mathrm{ANC}}^{\prime}$ tenga como referencia a un potencial LJ 12-6 (el cual es un modelo ampliamente estudiado) hacen de la teoría ANC una buena alternativa para utilizarse en este tipo de sistemas.

La versión $2 \mathrm{~S}$ de esta teoría permite relacionar los volúmenes medios de colisión $b^{*}$ y $\Lambda^{*}$, (ecs. 3.23 y 3.24 pág. 34, del sistema de estudio en términos de una referencia. Para valores fijos de $\mu^{*}, \alpha^{*}$ y $T^{*}$ del sistema se supone que tanto la rama repulsiva como atractiva del potencial $\psi_{\text {SMP }}$ son conformales a las ramas correspondientes de

$\psi_{\mathrm{LJ}}$; es decir, del potencial $\Phi_{\mathrm{ANC}}^{\prime}(S=1)$. En otras palabras, puede hallarse un potencial $\Phi_{\mathrm{ANC}}^{\prime}$ con parámetros efectivos que sea conformal a un potencial $\Phi_{\mathrm{SMP}}$ en un mismo estado.

Debido a que todos los integrantes de la familia de potenciales $\psi$ han sido construidos de tal forma que coincidan en su punto mínimo, los parámetros de energía y distancia $\left(\epsilon_{\mathrm{ef}}\right.$ y $\left.\delta_{\mathrm{ef}}\right)$ usados para tal efecto forman parte del sistema efectivo ANC. Por lo tanto, solo se requiere hallar las suavidades que hagan que el potencial $\psi_{\mathrm{LJ}}$, tanto en la zona repulsiva como en la atractiva, sea conformal al potencial $\psi_{\mathrm{SMP}}$ de interés y éstas se obtienen numéricamente. 
El procedimiento para hallar $S_{\mathrm{R}}$ y $S_{\mathrm{A}}$ parte de la definición integral de los volúmenes reducidos de colisión, ecs 3.23 y 3.24 , de la teoría ANC

$$
\begin{aligned}
b^{*} & =1-\frac{3}{e^{1 / \mathbb{T}^{*}}} \int_{0}^{1} d y y^{2} \mathrm{e}^{-\psi_{\mathrm{SMP}}\left(y, T^{*}\right) / \mathbb{T}^{*}} \\
\Lambda^{*} & =1+\frac{3}{\mathrm{e}^{1 / \mathbb{T}^{*}}-1} \int_{1}^{\infty} d y y^{2}\left[\mathrm{e}^{-\psi_{\mathrm{SMP}}\left(y, T^{*}\right) / \mathbb{T}^{*}}-1\right] .
\end{aligned}
$$

aplicados al potencial $\psi_{\mathrm{SMP}}$, y asegurándonos que dichos volúmenes cumplen las relaciones características

$$
\begin{aligned}
b^{*} & =1-S_{\mathrm{R}}+S_{\mathrm{R}} b_{0}^{\prime *} \\
\Lambda^{*} & =1-S_{\mathrm{A}}+S_{\mathrm{A}} \Lambda_{0}^{\prime *}
\end{aligned}
$$

con respecto al sistema de referencia. Las integrales anteriores distinguen dos temperaturas $T^{*}$ y $\mathbb{T}^{*}$; la primera de ellas corresponde al estado del sistema SMPs que se estudia, mientras que la segunda corresponde a la temperatura del sistema efectivo ANC que intenta reproducirlo ${ }^{12}$ Las integrales para los volúmenes $b^{*}$ y $\Lambda^{*}$ se resuelven numéricamente mientras que los volúmenes de referencia $b_{0}^{\prime *}$ y $\Lambda_{0}^{\prime *}$ se definen mediante las expresiones analíticas 3.49 y 3.50 dadas en la sección 3.5.4. pág. 40.

Las figuras 4.4 y 4.5 muestran algunos resultados numéricos de $S_{\mathrm{R}}$ y $S_{\mathrm{A}}$ obtenidos por el procedimiento antes mencionado. En ambos casos se muestra la tendencia de las suavidades cuando la temperatura aumenta y/o el momento dipolar disminuye.

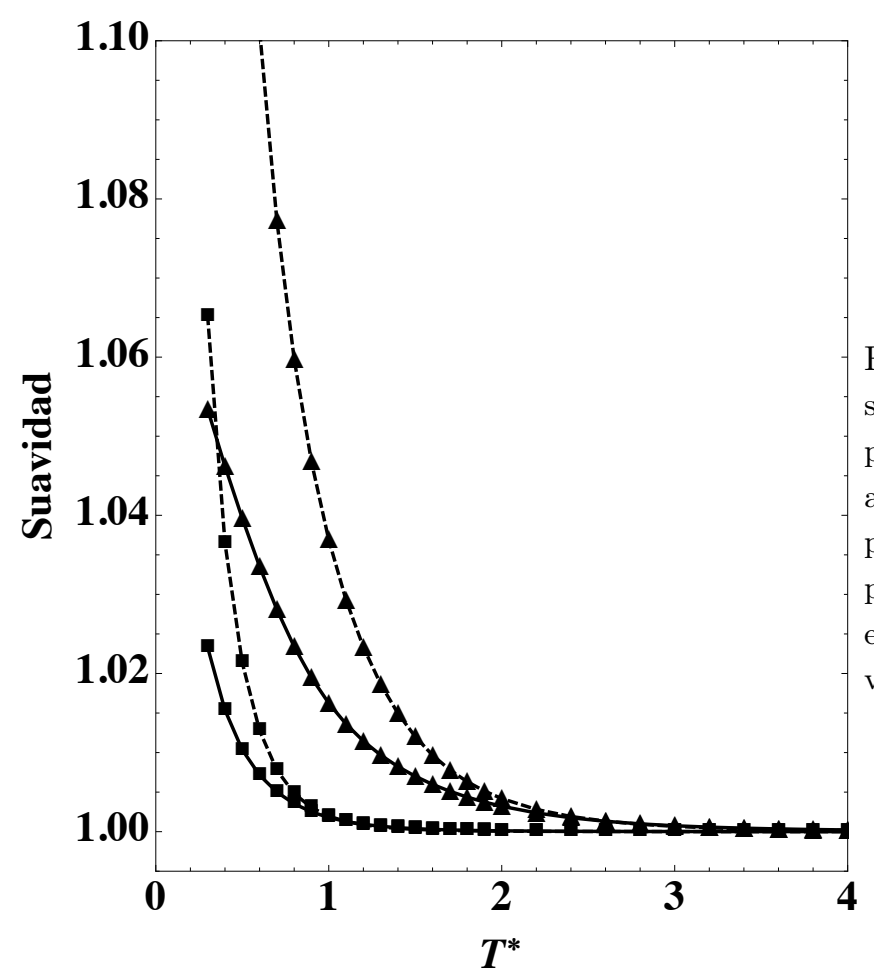

Figura 4.4: Comportamiento de los parámetros $S_{\mathrm{R}}$ (líneas sólidas) y $S_{\mathrm{A}}$ (líneas a trazos) en función de la temperatura para dos momentos dipolares: $\mu^{* 2}=2(\boldsymbol{\square})$ y $\mu^{* 2}=4(\boldsymbol{\Lambda})$, ambas a $\alpha^{*}=0.1$. Como puede observarse, la suavidad para la zona atractiva es mayor que la zona repulsiva. Los puntos son obtenidos vía el procedimiento mencionado en esta sección y las líneas fueron trazadas como ayuda a la vista.

A medida que la temperatura aumenta (ó el momento dipolar disminuye) se tiene que $S_{\mathrm{R}} \rightarrow 1$ y $S_{\mathrm{A}} \rightarrow 1$. Mientras más se alejen las suavidades de la unidad, mayor será la no-conformalidad entre los potenciales $\psi_{\mathrm{SMP}}$ y el $\psi_{\mathrm{LJ}}$. Cotejando las dos figuras simultáneamente se verifica fácilmente que para $\mu^{* 2} \leq 1$ y temperaturas $T^{*}>1$ los potenciales $\psi_{\mathrm{SMPs}}$ son conformales a un LJ. Una observación extra: De las mismas figuras se observa

\footnotetext{
${ }^{12}$ De hecho existen distintos sistemas efectivos ANC que pueden reproducir al fluido SMPs a una temperatura dada; sin embargo, se requiere que ellos cumplan la linealidad hallada en 4.30 y 4.31 exigida por dicha teoría.
} 
Figura 4.5: Comportamiento de los parámetros $S_{\mathrm{R}}$ (líneas sólidas) y $S_{\mathrm{A}}$ (líneas a trazos) en función de la intensidad

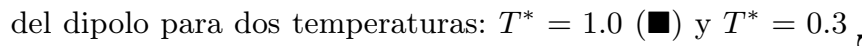
$(\boldsymbol{\Lambda})$, ambas a $\alpha^{*}=0.1$. Los puntos son obtenidos vía el

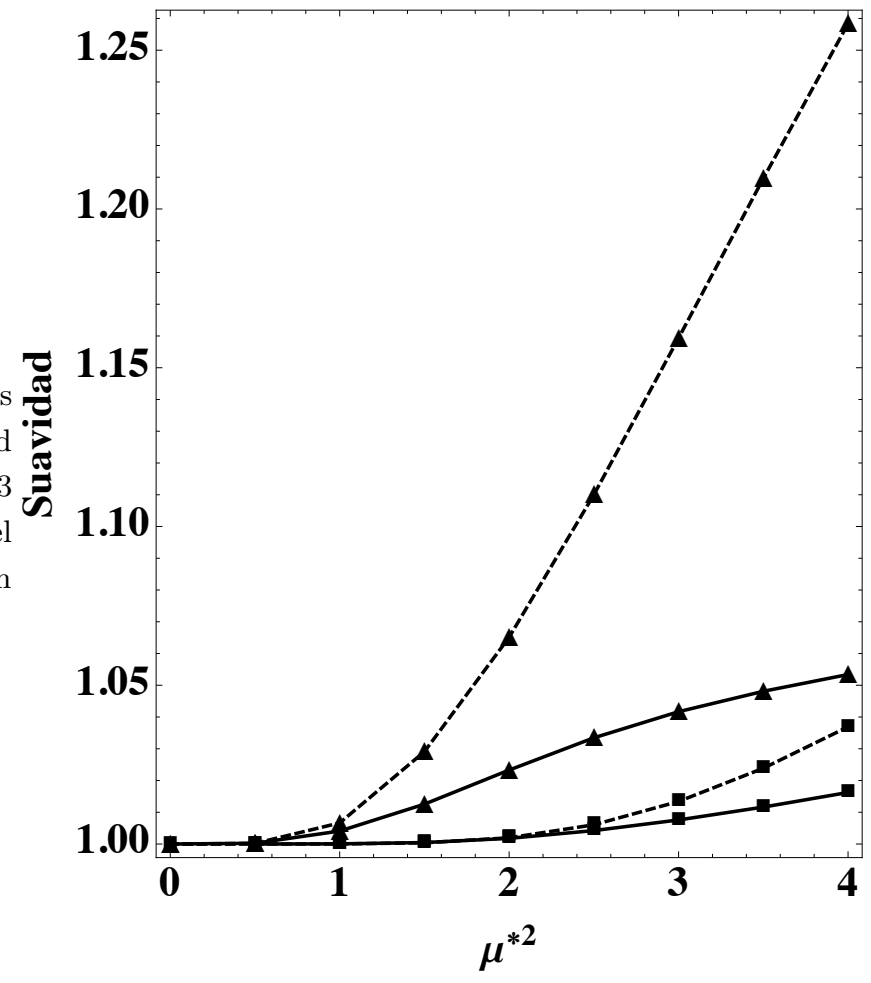
procedimiento mencionado en esta sección y las líneas fueron trazadas como ayuda a la vista.

que cuando el sistema de estudio se aleja de dicha conformalidad la diferencia entre los valores de $S_{\mathrm{R}}$ y $S_{\mathrm{A}}$ es muy notoria lo que indica que la teoría $\mathrm{ANC}$ en su versión $2 \mathrm{~S}$ resulta adecuada para detectar dichos cambios.

Este método permite corroborar los resultados observados en la figura 4.3(b) Aquellos potenciales $\psi_{\mathrm{SMP}}$ que se superponen al potencial $\psi_{\mathrm{LJ}}$ deben tener suavidades prácticamente iguales a la unidad y aquellos casos en los que ambos potenciales no se superponen se tiene que $S_{\mathrm{R}}>1$ y $S_{\mathrm{A}}>1$. La figura 4.6 presenta la diferencia entre usar un potencial efectivo LJ y uno tipo ANC para reproducir la función $\Phi_{\mathrm{SMPs}}^{*}$.

\subsection{Termodinámica efectiva para un gas SMP}

Como ya se ha mostrado, una función LJ puede utilizarse como un potencial efectivo para distintos estados de un sistema SMPs. El método puede extenderse y profundizar gracias al uso de la teoría ANC la cual introduce el concepto de suavidad que mide cuantitativamente la diferencia entre perfiles del potencial intermolecular con respecto a uno cuya termodinámica es conocida. En ambos casos el PEC establece que la termodinámica del sistema SMP esfericalizado puede representarse por cualquiera de estos dos potenciales dependiendo del estado que se esté estudiando.

Demostrada la conformalidad entre un potencial efectivo y el potencial esfericalizado $\Phi_{\mathrm{SMP}}$ (promedio configuracional de la interacción $\Phi_{\mathrm{SMP}}$ ), se propone ahora una equivalencia directa entre los sistemas SMP y el efectivo (LJ ó ANC). Con esto, las propiedades termodinámicas obtenidas con el sistema efectivo podrán ser cotejadas con aquellas obtenidas con el sistema SMP original.

\subsubsection{Potencial efectivo para un fluido SMP}

El método planteado al final de la seccion anterior permite obtener un potencial efectivo que reproduce al sistema SMP esfericalizado en un conjunto amplio de estados. Esta función efectiva puede ser del tipo LennardJones cuando se utilizan momentos dipolares pequeños siendo necesarios tan solo dos parámetros característicos, $\epsilon_{\text {ef }}$ y $\delta_{\text {ef. }}$ Cuando se trabaja con intensidades dipolares mayores es necesario incluir más parámetros y en este 


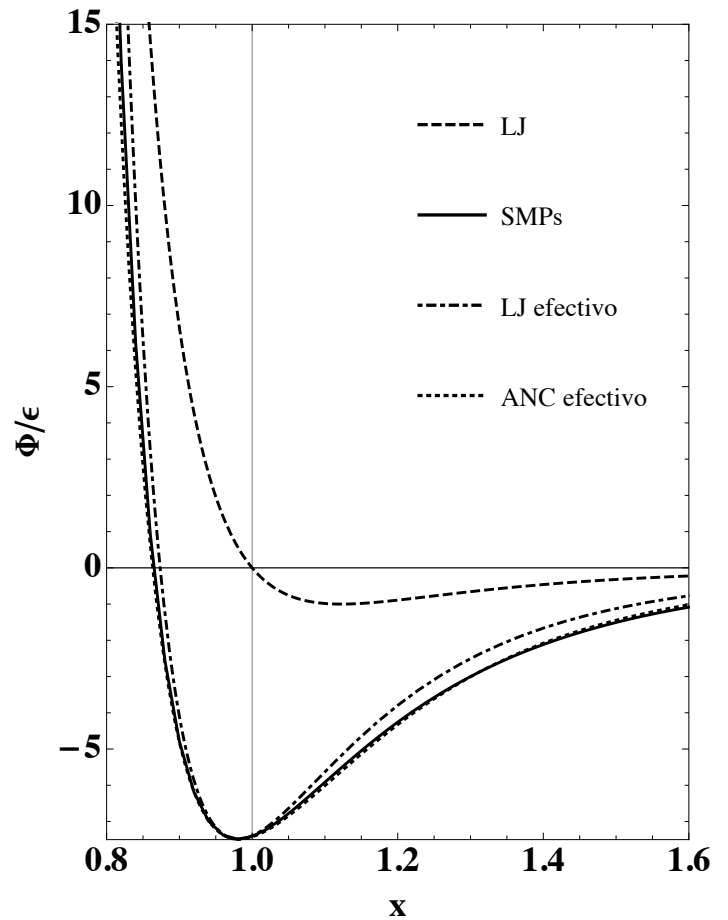

Figura 4.6: Potenciales efectivos para fluidos SMP a $T^{*}=0.3, \mu^{* 2}=4.0$ y $\alpha^{*}=0.1$. El potencial original $\Phi_{\mathrm{SMPs}}^{*}$ (línea sólida) se compara con los potenciales efectivos construidos en este trabajo. Para intensidades grandes del dipolo, el potencial efectivo tipo LJ con parámetros efectivos $\epsilon_{\mathrm{ef}} \mathrm{y} \delta_{\mathrm{ef}}$ no es conformal al potencial que se estudia. Utilizando un potencial efectivo tipo ANC (curva punteada) con $S_{\mathrm{R}}=1.085 \mathrm{y}$ $S_{\mathrm{A}}=1.208$ se empalman de mejor manera con el potencial esfericalizado. En la misma figura se presenta el potencial LJ a modo de comparación.

sentido el potencial ANC cumple ese objetivo usando el parámetro de suavidad para relacionar la forma del potencial de interés con una referencia.

Debido a que se ha considerado a la esfericalización del potencial $\Phi_{\mathrm{SMP}}$ como una función representativa de este sistema, se espera que el modelo efectivo pueda reproducir a un fluido SMP de manera adecuada a nivel del gas. En este sentido, los resultados obtenidos con nuestro sistema efectivo podrán constatarse con los diversos estudios existentes en la literatura que abordan los sistemas SM y SMP.

De manera general, el potencial efectivo para un fluido SMP estará dado por

$$
\Phi_{\mathrm{ef}}(r)=\Phi_{\mathrm{ANC}}^{\prime}\left(r ; \epsilon_{\mathrm{ef}}, \delta_{\mathrm{ef}}, S_{\mathrm{R}}, S_{\mathrm{A}}\right)
$$

donde se enfatiza el hecho de que es esféricamente simétrico y depende a lo más de cuatro parámetros característicos. La función $\Phi_{\text {ANC }}^{\prime}$ está dada por la ecuación 3.46 pág. 40 y como ya se ha mencionado es un caso especial de la teoría ANC. Además, cuando los parámetros suavidad cumplen la relación $S_{\mathrm{R}} \approx S_{\mathrm{A}} \approx 1$ el potencial anterior se reduce a una función LJ con una dependencia completa en el mínimo del potencial SMPs.

La aplicación de esta definición puede resumirse de la siguiente manera: a un sistema SMP en un estado dado le corresponde un potencial SMPs representado por la ecuación 4.20, pág. 50]. El mínimo de este potencial esfericalizado (parámetros $\epsilon_{\text {ef }}$ y $\delta_{\text {ef }}$ ) se utiliza para reescalarlo y encontrar su respectivo potencial $\psi_{\mathrm{SMP}}$ (ec. 4.27 pág. 53 el cual coincidirá en el mínimo con el potencial $\psi$ del LJ. En el supuesto de que ambos potenciales coincidan en su perfil, la ec. 4.32 en su forma Lennard-Jones deberá ser suficiente para utilizarse como el potencial efectivo del sistema original SMP. En caso contrario, cuando los perfiles no se superpongan se sigue el desarrollo ANC mostrado en la sección 4.4.6 obteniéndose dos nuevos parámetros $S_{\mathrm{R}}$ y $S_{\mathrm{A}}$ por lo cual se debe usar el caso general del potencial efectivo dado por 4.32 .

El procedimiento anterior no conlleva complejidad alguna y la obtención de cada uno de los parámetros efectivos se realiza de forma bastante sencilla. La única "desventaja" recae en el hecho de que debe recorrerse el mismo camino para cada nuevo estado. Esto realmente se supera fácilmente creando rutinas básicas por computadora que salven ese inconveniente. A pesar de ello, se ha optado por generar expresiones que representen dichos parámetros en términos de las propiedades del sistema de estudio; es decir, de la temperatura y de las 
intensidades electrostáticas del sistema dentro de intervalos de nuestro interés. Esto permitirá manejar a las ecuaciones efectivas en términos tan solo de parámetros macroscópicos, mientras que los microscópicos estarán presentes de manera implícita en la expresion final. Una ventaja extra en la construcción de estas expresiones resulta en que pueden analizarse una gran cantidad de estados de manera simultánea. Este tipo de funciones han sido obtenidas para los parámetros efectivos de distancia y energía; es decir, aquellos que ubican el mínimo del potencial esfericalizado y a continuación son presentadas.

\subsubsection{Funciones de escalamiento para sistemas SMPs}

La implementación de funciones que caractericen al mínimo, $\epsilon_{\mathrm{ef}} \mathrm{y} \delta_{\mathrm{ef}}$, del potencial $\Phi_{\mathrm{SmPs}}$ permite implementar técnicas rápidas y sistemáticas con las cuales calcular las propiedades termodinámicas del sistema polarizable en cualquier estado requerido.

Debido a que las cantidades con las que se trabaja han sido reducidas con los parámetros del kernel, $\epsilon$ y $\sigma$, la coordenada del potencial $\Phi_{\mathrm{SMPs}}^{*}$ en su punto mínimo estará dada por $F_{\epsilon}$ y $F_{\delta}{ }^{13}$ de tal forma que los parámetros efectivos tienen la forma

$$
\begin{aligned}
& \epsilon_{\mathrm{ef}}=\epsilon F_{\epsilon} \\
& \delta_{\mathrm{ef}}=\sigma F_{\delta} .
\end{aligned}
$$

Entonces, todo recae en la construcción de funciones que reproduzcan estos valores para cualquier estado de análisis en este trabajo; así, se partirá de un conjunto de datos $\left(F_{\epsilon}, F_{\delta}\right)$ obtenidos por minimización de la función $\Phi_{\mathrm{SMPs}}^{*}$ y posteriormente se buscará un ajuste adecuado basado en la información física de dichos sistemas. El método empleado ha hecho uso de un conjunto de datos discretos en intervalos de 0.5 tanto para $\mu^{*}$ como para $T^{*}$ y de 0.02 para $\alpha^{*}$. Se ha propuesto que el ajuste para este conjunto de datos sea 14

$$
\begin{aligned}
& F_{\epsilon}\left(T^{*}, \mu^{*}, \alpha^{*}\right)=f_{\epsilon}+g_{\epsilon} \alpha^{*} \\
& F_{\delta}\left(T^{*}, \mu^{*}, \alpha^{*}\right)=f_{\delta}+g_{\delta} \alpha^{*}
\end{aligned}
$$

donde las funciones $f_{x}$ representan el mínimo para el caso SM y las funciones $g_{x}$ aparecen cuando se incluye la inducción en la interacción principal. La inclusión de la polarizabilidad se hace en congruencia con la ecuación 4.9, donde el cambio en el mínimo de la función $\Phi_{\mathrm{SMPs}}^{*}$ incluye un término proporcional a $\alpha^{*}$.

Para expresar de manera adecuada estos ajustes, se ha intentado mantener la misma forma funcional de las expresiones $F_{\epsilon}$ y $F_{\delta}$ presentadas en las ecuaciones 4.23 y 4.24), respectivamente. Nótese, además, que el potencial $\Phi_{\mathrm{SMPs}}^{*}$ está conformado por tres distintas contribuciones energéticas: la del kernel, la cinética $T^{*}, \mathrm{y}$ la electrostática. Ésta última maneja la combinación $\mu^{* 2} / T^{*}$, la cual mide la razón entre las energías electrostática y cinética como puede verse de la ec 4.20). Sin embargo, el factor extra $T^{*}$ (el cual mide la razón entre la energía cinética y la energía del kernel) que multiplica al logaritmo natural en dicha ecuación muestra que el potencial esfericalizado no puede expresarse tan solo en términos de la variable $\mu^{* 2} / T^{*}$ sino que es necesario incluir coeficientes dependientes de la temperatura.

El primer ajuste realizado fue para el caso SM donde las funciones $f_{x}$ toman la forma

$$
\begin{aligned}
& f_{\epsilon}=\frac{1+\mathcal{A}_{1} \mu^{* 4} / T^{* 2}+\mathcal{A}_{2} \mu^{* 8} / T^{* 4}}{1+\mathcal{A}_{3} \mu^{* 4} / T^{* 2}}, \\
& f_{\delta}=\frac{2^{1 / 6}+\mathcal{A}_{4} \mu^{* 4} / T^{* 2}+\mathcal{A}_{5} \mu^{* 8} / T^{* 4}}{1+\mathcal{A}_{6} \mu^{* 4} / T^{* 2}},
\end{aligned}
$$

y los numeradores para $\mu^{* 2} \leq 1$ son muy cercanos a los encontrados por Ávalos et. al. [11]; los denominadores son necesarios para que el ajuste sea exitoso para $\mu^{* 2} \leq 4$. Los coeficientes $\mathcal{A}_{i}$ son funciones dependientes de $T^{*}$

\footnotetext{
${ }^{13}$ Ver sección 4.4 .4

${ }^{14}$ Para evitar alguna confusión los símbolos $F_{x}$ representarán tanto al dato puntual como a las funciones ahora manejadas; el contexto indicará cual de los dos conceptos se está ocupando.
} 
y su forma exacta se presenta en el apéndice B.3.1. pág. 115 . Su comportamiento con respecto a $\mu^{* 2}$ es mostrado por las líneas continuas de la figura 4.7 para distintas temperaturas. Como puede verse, el cambio tanto en la energía como en la posición se acrecenta a medida que la temperatura disminuye ó el momento dipolar aumenta; un efecto que es mucho mayor para el primer caso que para el segundo.

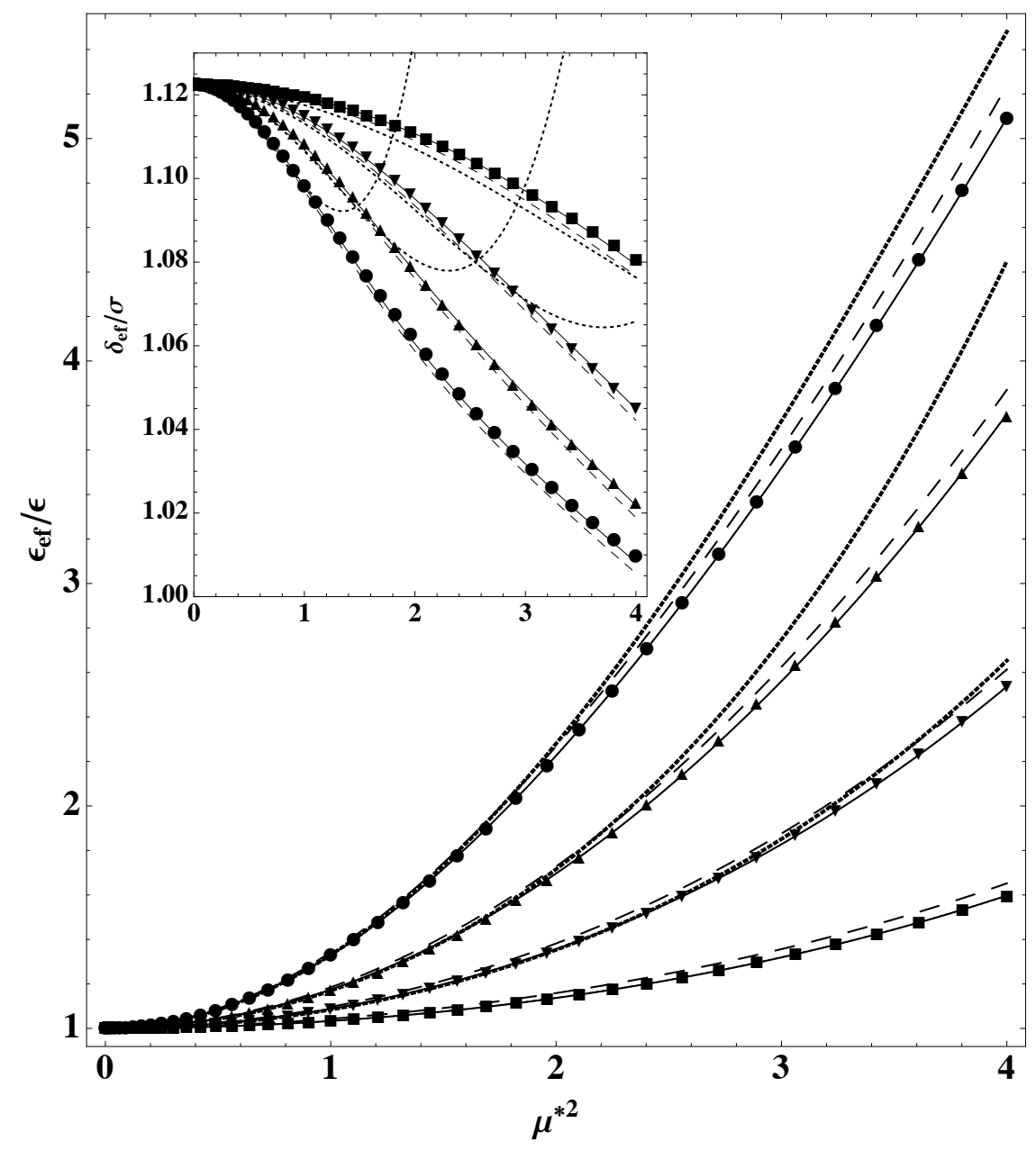

Figura 4.7: Energía relativa $\epsilon_{\text {ef }} / \epsilon$ y diámetro $\delta_{\text {ef }} / \sigma$ (recuadro) para un fluido SMP y su dependencia con la temperatura y el momento dipolar (curvas continuas). El efecto de la polarizabilidad se observa en las curvas a trazos para un valor de $\alpha^{*}=0.1$ y a las temperaturas reducidas: $T^{*}=0.5(\boldsymbol{\square}), 1.0(\boldsymbol{\nabla}), 2.0(\mathbf{\Delta})$ y $5.0(\bullet)$. Las líneas provienen de los ajustes mencionados en el cuerpo principal, ecs. (4.35) y 4.36 y los puntos son algunos ejemplos obtenidos por minimización directa de $\Phi_{\mathrm{SMPs}}^{*}$. En la misma figura se presenta (curvas punteadas) el potencial efectivo Lennard-Jones dado por las ecuaciones 4.23 y 4.24 cuando $\alpha^{*}=0.1$.

Para el caso polarizable, las funciones $g_{x}$ se construyen tomando en cuenta la dependencia lineal en $\alpha^{*}$ dadas por las ecuaciones 4.35 y 4.36. Así, al conjunto de datos SMP solo le es restado el efecto SM, ecs 4.37) y 4.38, quedando tan solo con el efecto debido a la inducción electrostática. De esta manera, el ajuste se facilita usando funciones de la forma

$$
\begin{aligned}
g_{\epsilon} & =\mathcal{B}_{1} \frac{\mu^{* 2}}{T^{*}}+\mathcal{B}_{2} \frac{\mu^{* 4}}{T^{* 2}} \\
g_{\delta} & =\mathcal{B}_{3} \frac{\mu^{* 2}}{T^{*}}+\mathcal{B}_{4} \frac{\mu^{* 4}}{T^{* 2}}
\end{aligned}
$$

donde los coeficientes $\mathcal{B}_{i}$ también son funciones dependientes de $T^{*}$ y su forma exacta se presenta en el apéndice 
B.3.1. pág. 115. El efecto en el mínimo del potencial SMPs al ser sumadas a las funciones $f_{x}$, ecs 4.33 y (4.34, se muestra en las cusrvas a trazos de la figura 4.7 para $\alpha^{*}=0.02$. El efecto de la polarizabilidad sobre el potencial esfericalizado SMP es pequeño para momentos dipolares pequeños, como se muestra en la figura; el cambio en la energía y posición del mínimo de $\Phi_{\mathrm{SMPs}}^{*}$ con respecto a la polarizabilidad es muy similar a como lo hace con el momento dipolar. Debido a que el efecto de las fuerzas electrostáticas sobre la forma de $\psi_{\mathrm{SMP}}(y)$ es despreciable para temperaturas grandes y cobra relevancia cuando disminuye la temperatura, las funciones $F_{\epsilon}$ y $F_{\delta}$ contienen mucha de la información acerca de la influencia de la interacción dipolo-dipolo sobre las propiedades del sistema. Como ya se mencionó, la forma racional de estas funciones y la inclusión de coeficientes dependientes de $T^{*}$ fueron necesarias para representar adecuadamente su comportamiento para valores grandes de $\mu^{* 2} / T^{*}$.

En la misma gráfica se muestran los parámetros efectivos para la aproximación tipo Lennard-Jones dada por la ecuación 4.22 tomando solo los primeros términos del potencial esfericalizado $\Phi_{\mathrm{SMPs}}^{*}$. Como se ve, a temperaturas altas la aproximación resultante sigue la tendencia correcta; sin embargo, a medida que la temperatura disminuye (y el momento dipolar aumenta) los valores se alejan bastante de los resultados exactos ubicados por las líneas continuas.

Las ecuaciones 4.35 y 4.36 están diseñadas para localizar el mínimo del potencial SMPs para valores $\mu^{* 2} \leq 4$ y $\alpha^{*} \leq 0.1$, por lo que su aplicación para intensidades mayores tanto en $\mu^{*}$ como en $\alpha^{*}$ se tomará como un resultado puramente cualitativo. Con estas nuevas definiciones la ecuación 4.32 puede reescribirse como

$$
\Phi_{\mathrm{ef}}(r ; T, \mu, \alpha)=\Phi_{\mathrm{ANC}}^{\prime}\left(r ;\left[\epsilon_{\mathrm{ef}}\right],\left[\delta_{\mathrm{ef}}\right], S_{\mathrm{R}}, S_{\mathrm{A}}\right)
$$

donde se enfatiza el hecho de haber incluido las funciones $\epsilon_{\mathrm{ef}}$ y $\delta_{\mathrm{ef}}$. Las mismas consideraciones hechas para 4.32 también son aplicables a esta nueva definición. Estas dos ecuaciones serán utilizadas regularmente cuando se tengan que hacer cálculos de propiedades termodinámicas para distintos sistemas en múltiples estados.

Demostrada la conformalidad entre estos dos sistemas (el efectivo y el SMPs) el PEC podría ser empleado para hallar las propiedades termodinámicas del sistema esfericalizado en términos del sistema efectivo. Sin embargo, si se propone que el potencial esfericalizado es una excelente representación del sistema SMP original, entonces la aplicación del PEC se enfocará en obtener la termodinámica del sistema SMP, de forma aproximada, a partir del potencial efectivo, ec. 4.41). La aplicación de dicho principio en el cálculo de los primeros coeficientes viriales para el sistema SMP y su extensión a densidades mayores se muestra a continuación.

\subsubsection{Segundo coeficiente virial efectivo para un fluido SMP}

De acuerdo al principio de estados correspondientes, conocida la forma analítica de $B_{\mathrm{ANC}}$ en su forma modificada, ec. (3.48 pág. 40), solo basta sustituir los parámetros moleculares de dicha expresión por $\delta \rightarrow \delta_{\text {ef }} \mathrm{y}$ $\epsilon \rightarrow \epsilon_{\mathrm{ef}}$, obtenidos en la sección anterior, para finalmente reducir la ecuación con los parámetros $\epsilon$ y $\sigma$ del kernel esférico de la interacción SMP original; el resultado final serê:

$$
B_{\mathrm{ef}}^{*}\left(T^{*}\right)=\frac{F_{\delta}^{3}}{\sqrt{2}} B_{\mathrm{ANC}}^{*}\left(\frac{T^{*}}{F_{\epsilon}}\right)
$$

donde $B_{\mathrm{ef}}^{*}=B_{\mathrm{ef}} / \sigma^{3}$ y $B_{\mathrm{ANC}}^{*}=B_{\mathrm{ANC}} / \sigma^{3}$ y los factores extras provienen de haber reducido con los parámetros efectivos del kernel esférico; por ello la aparición de los factores reducidos $F_{\epsilon}$ y $F_{\delta}{ }^{16}$ que de manera implícita

${ }^{15}$ Este resultado se verifica fácilmente al integrar directamente $B_{\text {ef }}$, ec. 2.21, usando el potencial 4.32,

$$
B_{\mathrm{ef}}\left(T, \mu, \alpha ;\left[\Phi_{\mathrm{ef}}\right]\right)=2 \pi \int d r r^{2}\left[1-e^{-\Phi_{\mathrm{ef}} / k_{\mathrm{B}} T}\right],
$$

y empleando cualquiera de los métodos desarrollados en el apéndice A.2 arrojando la misma expresión presentada en la ecuación 4.43 .

${ }^{10} \mathrm{En}$ los casos en que el potencial LJ sea un excelente potencial efectivo la ec 4.43 se reduce a

$$
B_{\mathrm{ef}}^{*}\left(T^{*}\right)=\frac{F_{\delta}^{3}}{\sqrt{2}} B_{\mathrm{LJ}}^{*}\left(\frac{T^{*}}{F_{\epsilon}}\right)
$$


incluyen el carácter electrostático del sistema SMP: $\mu^{*}$ y $\alpha^{*}$.

La validez de $\Phi_{\text {ef }}$ como potencial efectivo capaz de reproducir al potencial $\Phi_{\mathrm{SMP}}$ comienza exigiendo que $B_{\text {ef }}$ concuerde con el correspondiente segundo coeficiente virial para el fuido SMP. Datos de simulación y expresiones analíticas en forma de series infinitas para el cálculo de $B_{\mathrm{SMP}}$ se encuentran disponibles en la literatura [33-35] y pueden ser comparadas con nuestro resultado efectivo; ejemplos de ello son mostrados en las figuras 4.8(a) (sistema SM) y 4.8(b) (sistema SMP) donde se presenta la dependencia con $T^{*}$ para distintos momentos dipolares.

En el cálculo del segundo coeficiente virial para $\mu^{* 2} \leq 4$ se usan las funciones $F_{\epsilon}$ y $F_{\delta}$ dadas por 4.35 y 4.36), mientras que para intensidades mayores se utiliza el respectivo mínimo de $\Phi_{\text {SMPs }}^{*}$ para cada uno de los estados requeridos; estos últimos casos sirven para analizar el concepto de conformalidad para sistemas SMP a intensidades dipolares mayores. En ambas figuras los resultados efectivos son comparados con aquellos obtenidos vía analítica [33] y en el caso SM también se incluyen cálculos de Vega et. al. [35] obtenidos vía simulación de MC de dos centros Lennard-Jones dipolares. Para el fluido SM polarizable $B_{\mathrm{SMP}}^{*}$, se utiliza una polarizabilidad mediana de $\alpha^{*}=0.1$ y el resultado analítico se obtiene con la ec. A.35 presentada en el apéndice. Las integrales angulares $h_{j}$ que aparecen en dicha referencia han sido resueltas como parte de este trabajo y escritas como una suma infinita, ver ecuación (B.15 pág. 114.

Como puede observarse en ambas figuras, el cálculo del segundo coeficiente virial mediante el uso de un potencial efectivo (ANC) arroja excelentes resultados al compararlos con los obtenidos por medios analíticos y de simulación, inclusive para momentos dipolares mayores a los establecidos. Para momentos dipolares pequeños $\left(\mu^{* 2} \leq 1\right)$ el potencial LJ basta para obtener resultados adecuados; sin embargo, a medida que el momento dipolar aumenta se comienzan a presentar desviaciones con respecto al dato correcto lo que indica una pérdida de conformalidad entre el potencial LJ y el SMP. Dicha conformalidad se recupera cuando se utiliza un potencial efectivo ANC aplicado para el cálculo del resto de las curvas $\left(\mu^{2} \geq 1\right)$ con resultados satisfactorios. Además del momento dipolar ${ }^{17}$ la temperatura es factor fundamental para lograr la conformalidad entre los sistemas involucrados. Así, como ya se había mostrado, la combinación temperatura baja-momento dipolar grande hace que se pierda dicha conformalidad, lo que provoca que el potencial efectivo sea cuantitativamente inadecuado. Lo anterior se ilustra de mejor manera observando la curva extrema derecha $\left(\mu^{* 2}=25, \alpha^{*}=0.1\right)$ de la figura 4.8(b) para temperaturas grandes el potencial efectivo logra resultados cercanos al analítico lo cual se pierde cuando la temperatura disminuye, rescatando tan solo el efecto cualitativo.

Si bien en la generación del potencial efectivo $\Phi_{\text {ef }}$, se parte de la igualdad de segundos coeficientes viriales entre el sistema esfericalizado (vinculado al potencial $\Phi_{\mathrm{SMPs}}$ ) y el sistema de interés SMP (vinculado al $\Phi_{\mathrm{SMP}}$ ), esto no es influyente en los resultados mostrados arriba. Es decir, el hecho de haber construido $\Phi_{\text {ef }}$ partiendo de la ecuación 4.12 tuvo como objetivo principal el eliminar el atributo orientacional del potencial original y no el de reproducir con exactitud el coeficiente virial estudiado. Sin embargo, sí hay algo implícito en tal ecuación, el potencial fue diseñado considerando tan solo interacciones moleculares por pares. Así, es de esperarse que cualquier otra propiedad que involucre las interacciones entre más de dos partículas no sea reproducida adecuadamente. En las siguientes líneas mostraremos que en el caso del tercer coeficiente virial $C(T)$, a medida que la densidad aumenta y/o la temperatura disminuya el presente potencial efectivo no reproduce el efecto esperado. Sin embargo, dicho resultado abre la puerta a la identificación efectiva de aquél efecto debido a la presencia de una tercer partícula dipolar e incluirlo en nuestro sistema efectivo.

\subsubsection{Efecto de frustración}

Se puede inferir de lo anterior que la presión de un gas SMP diluido para momentos dipolares pequeños puede obtenerse adecuadamente con la ayuda del potencial efectivo de $\Phi_{\mathrm{SMP}}$. Sin embargo, en la construcción

\footnotetext{
${ }^{17}$ Aunque la polarizabilidad también es un factor importante en la existencia de conformalidad entre los sistemas efectivo y real, su importancia con respecto a $\mu^{*}$ y $T^{*}$ puede considerarse menor.
} 


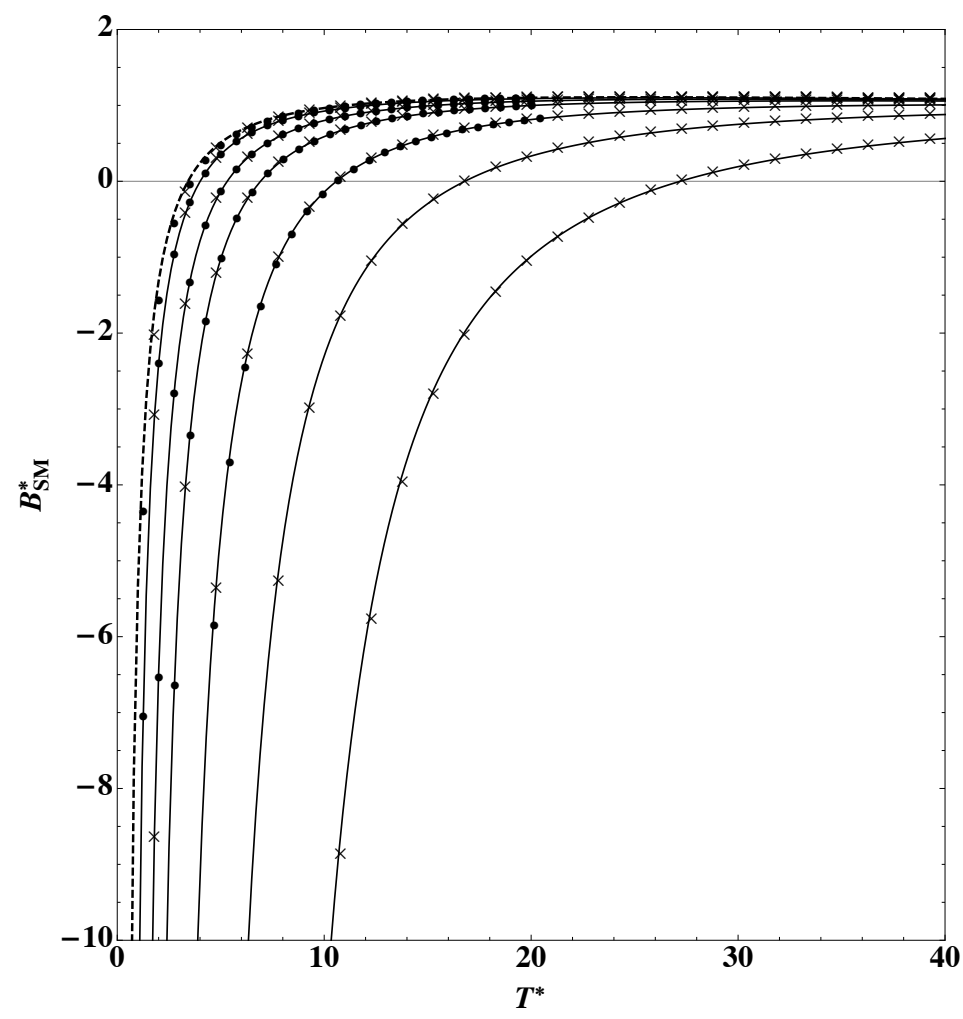

(a)

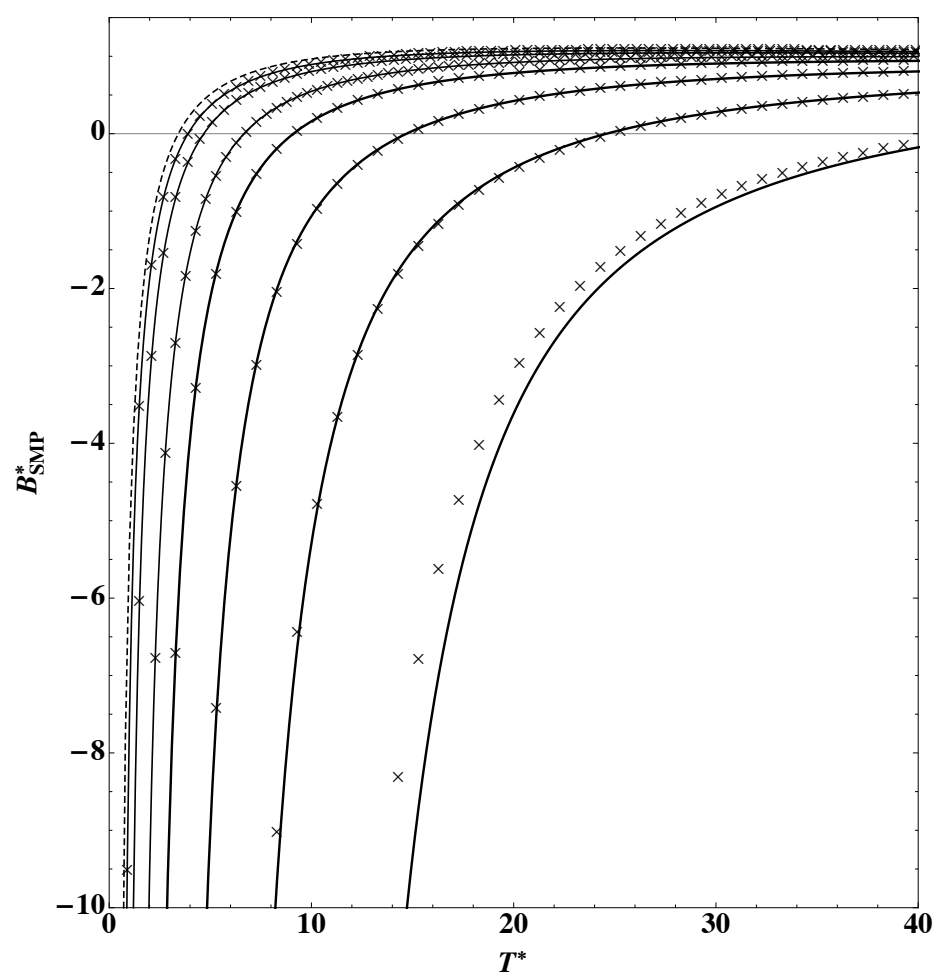

Figura 4.8: Predicción de $B_{\mathrm{SMP}}^{*}\left(T^{*}\right)$ con interacciones efectivas: (a) $\alpha^{*}=0.0$ y (b) $\alpha^{*}=$ 0.1. Para ambas gráficas, de izquierda a derecha, $\mu^{* 2}=1,2,4,6,10,16$ y 25 ; los primeros dos casos son obtenidos usando los parámetros efectivos $\epsilon_{\text {ef }}$ y $\delta_{\text {ef }}$, ecs. 4.33 y 4.34. En el resto de los casos se emplean los mínimos $\epsilon_{\text {ef }} \mathrm{y}$ $\delta_{\text {ef }}$, obtenidos numéricamente, y posteriormente se aplica el método explicado en el cuerpo principal del trabajo. Los datos incluidos en la gráfica provienen de dos fuentes distintas: simulación MC realizado por Vega et. al. [35] del segundo coeficiente virial para dos centros LJ con dipolos puntuales (puntos) y mediante la expresión analítica del coeficiente presentada en el apéndice (cruces) [33, 34]. A modo de referencia, se incluye el segundo coeficiente virial cuando la interacción dipolar es nula: $\mu^{* 2}=0$ (línea a trazos a la izquierda).

(b) 
de este potencial tan solo se han involucrado interacciones entre dos partículas lo que presupone que la presión debida al sistema efectivo se alejará de la del sistema SMP a medida que la densidad aumente. Es decir, se puede esperar que el siguiente término en el desarrollo del virial, el tercer coeficiente virial efectivo $C_{\text {ef }}$, obtenido vía el potencial $\Phi_{\text {ef }}$ no arroje el valor correcto en la obtención de la presión.

Expresiones analíticas del tercer coeficiente virial existen para el potencial LJ. Por lo tanto, el primer paso conveniente será el trabajar con intensidades del momento dipolar y temperaturas que permitan que un sistema SMP sea conformal al potencial LJ. El potencial efectivo tendrá esta forma y sólo serán requeridos parámetros de energía y distancia, $\epsilon$ y $\delta$, para construirlo. Si se supone que el potencial efectivo reproduce el tercer coeficiente virial de la misma manera que lo hace para el segundo, entonces $C_{\text {ef }}$ puede escribirse de manera similar a la ecuación 4.43); es decir

$$
C_{\mathrm{ef}}^{*}\left(T^{*}, \mu^{*}, \alpha^{*}\right)=\frac{F_{\delta}^{6}}{2} C_{\mathrm{LJ}}^{*}\left(\frac{T^{*}}{F_{\epsilon}}\right)
$$

donde $C_{\mathrm{LJ}}^{*}$ es el tercer coeficiente virial reducido del sistema Lennard-Jones (ver A.2.3 en el apéndice). La figura 4.9 refleja la inconsistencia al usar el sistema efectivo $\Phi_{\text {ef }}$ en la construcción de $C_{\text {ef }}$ para un caso SM.

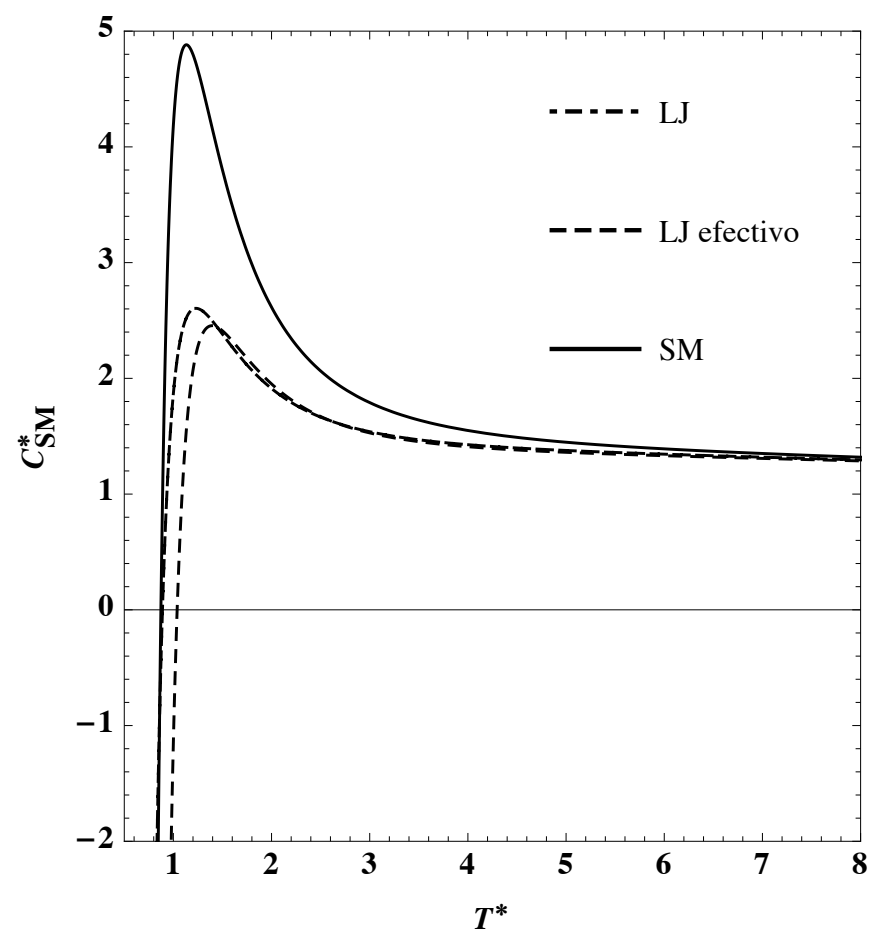

Figura 4.9: Tercer coeficiente virial para un fluido SM: vía potencial efectivo, ec. 4.45, y el cálculo directo $C_{\mathrm{SM}}^{*}($ línea sólida), ec. A.38, para $\mu^{* 2}=1.0$. También, a modo de comparación, la figura incluye el tercer coeficiente virial del fluido LJ.

En dicha figura se observa que $C_{\mathrm{ef}}^{*}$ ofrece valores muy bajos con respecto al tercer coeficiente virial verdadero de un fluido SM $C_{\mathrm{SM}}^{*}$, cuya expresión analítica [36] aparece en el apéndice, ec. [A.38), y la cual contiene una contribución positiva significativa (repulsiva) sobre los valores de la interacción Lennard-Jones. En términos cualitativos esto significa que cuando se construye el tercer coeficiente las contribuciones (negativas) sobre el potencial efectivo son muy grandes para densidades mayores. Esta discrepancia se explica debido a las correlaciones de largo alcance generadas al involucrar un tercer dipolo, a densidades ya moderadas. A modo de ilustración, tómese la configuración existente entre un par de dipolos en la presencia de un tercero (véase la figura 4.10; ; todos con una intensidad dipolar de $\mu^{* 2}=1.0$ y una distancia unitaria entre ellos. La configuración más estable entre las tres partículas es mostrada en esa figura con un mínimo energético, por cada par de dipolos, de $-1.25 \epsilon$. Este valor es menor a aquél obtenido cuando el tercer dipolo no está presente con una energía de $-2 \epsilon$ y un arreglo cabeza-cola entre ellos. Es decir, la configuración más estable entre dos dipolos se ve alterada cuando se presenta la tercera partícula; a este efecto se le conoce en la literatura como "frustración" e incide directamente en distintas áreas de la teoría de fluidos dipolares [37-41] áun para intensidades dipolares 
moderadas $\left(\mu^{* 2} \leq 3\right)$.

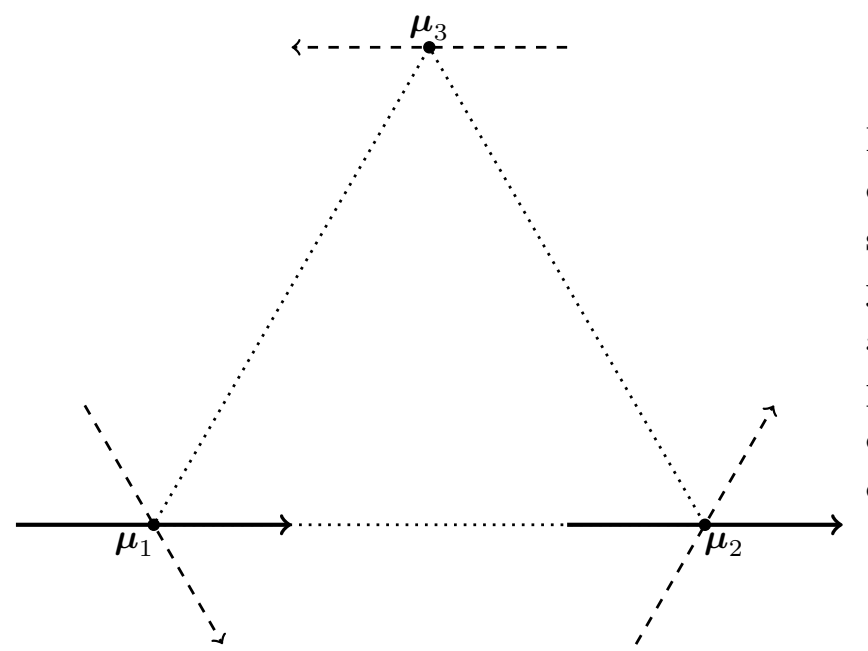

Figura 4.10: Efecto sobre un par de dipolos, $\boldsymbol{\mu}_{1}$ y $\boldsymbol{\mu}_{2}$, debido a la presencia de un tercero, $\boldsymbol{\mu}_{3}$. Supónganse las siguientes magnitudes de los dipolos: $\mu_{1}^{*}=\mu_{2}^{*}=\mu_{3}^{*}=1$, $\mathrm{y}$ las distancias reducidas entre ellos: $x_{12}=x_{13}=$ $x_{23}=1$. La configuración más estable para las tres partículas se muestra por las flechas a trazos con energía de interacción por pares de $-1.25 \epsilon$, a diferencia de la configuración más estable entre dos dipolos solitarios (dipolos 1 y 2 , líneas continuas) con energía $-2 \epsilon$.

Dicho efecto de frustración aparece en cualquier arreglo (no colineal en el caso del ejemplo anterior) por lo que se puede intuir que el potencial $\Phi_{\mathrm{SMPs}}$, proveniente de promediar angularmente todas las configuraciones posibles entre dos dipolos -ahora en presencia de un tercer cuerpo- debe tener una profundidad menor a la indicada por $\epsilon_{\mathrm{ef}}$, contenido en el potencial efectivo $\Phi_{\mathrm{ef}}$, ec. 4.32 . Esta sobrestimación del efecto dipolar en nuestro potencial efectivo, al no incluir la frustración a densidades moderadas, se refleja en el cálculo del tercer coeficiente virial obtenido arriba.

Así, el efecto de frustración aparece a nivel del tercer coeficiente virial y para tomarlo en cuenta debe ser analizado desde otra perspectiva. En este coeficiente se puede separar la contribución debida a la interacción LJ de aquella proveniente de la parte polar, tal que esta segunda parte se haga cero cuando $\mu^{*}=0$. Entonces, de acuerdo a lo anterior, cuando se toman en cuenta tres o más partículas la inclusión de la contribución polar debe resultar en una repulsión extra o una disminución en la atracción a la que se obtiene en el caso analizado en las secciones previas. La manera más sencilla de considerar esta disminución en la atracción es reducir la profundidad $\epsilon_{\mathrm{ef}}$ del pozo del potencial efectivo de manera tal que el efecto neto sea la reducción de la interacción dipolo-dipolo promedio. Ahora, debido a que la profundidad del potencial efectivo $\epsilon_{\mathrm{ef}}$ se introduce a nivel del segundo coeficiente virial, se propone que el factor de corrección debe ser proporcional a la densidad por lo que contribuirá finalmente al nivel de $C(T)$. También se supone que dicho efecto de frustración debe disminuir o saturarse a medida que la densidad aumente; esto puede verse por ejemplo, cuando se consideran cuatro dipolos; ellos pueden asociarse en dos pares, cada uno formado por dipolos antiparalelos.

Así, se puede suponer que el efecto de frustración puede ser incorporado incluyendo un factor adimensional $j_{\epsilon}$, dependiente de la densidad reducida, que afecta la energía efectiva dada en la ec. 4.33 tal que

$$
\begin{aligned}
\epsilon_{\mathrm{ef}} & =\epsilon F_{\epsilon} j_{\epsilon} \\
\delta_{\mathrm{ef}} & =\sigma F_{\delta}
\end{aligned}
$$

los cuales adoptaremos, a partir de este momento, como los nuevos parámetros efectivos sustituyendo a los definidos en las ecuaciones 4.33 y 4.34.

Puede proponerse un modelo sencillo para $j_{\epsilon}$ suponiendo que cuando se está en la fase del gas dicho efecto varíe linealmente con la densidad y que además $j_{\epsilon} \rightarrow 1$ cuando $\rho \rightarrow 0$; de esta forma la propuesta inicial será

$$
j_{\epsilon}=1-\gamma_{\epsilon} \rho^{*}
$$

donde $\rho^{*}=\rho \sigma^{3}$ es la densidad reducida del sistema y $\gamma_{\epsilon}$ es el parámetro que, acorde a la densidad, nos indica la cantidad en que disminuye el factor $\epsilon_{\mathrm{ef}}$ dado por la ecuación (4.33). 
Además, como se ha mencionado anteriormente, existe una razón para esperar que dicho efecto deba saturarse para densidades más altas. Tomando en cuenta ello, se propone una generalización a la ecuación anterior, incluso para estudiar sistemas a altas densidades, dada por

$$
j_{\epsilon}=1-k_{\epsilon} \tanh \left(\frac{\rho^{*}}{\rho_{s}^{*}}\right)
$$

donde $k_{\epsilon}$ y $\rho_{s}^{*}$ serán parámetros dependientes de la temperatura y del carácter electrostático del sistema. De dicha ecuación se puede comprobar fácilmente que a densidades bajas se tiende a la ecuación (4.48) de tal forma que $\gamma_{\epsilon}=k_{\epsilon} / \rho_{s}^{*}$. En particular, $1-k_{\epsilon}$ determinará la magnitud máxima de la frustración y $\rho_{s}^{*}$ determina la densidad a la cual el sistema dipolar se satura y el efecto se estabiliza.

En la siguiente sección se muestra que el potencial efectivo 4.41], con la inclusión de un efecto de frustración, es compatible con la expresión completa del tercer coeficiente virial tal que la dependencia de $\gamma_{\epsilon}$ con $\mu^{*}$ y $T^{*}$ puede ser determinada a bajas densidades; esto abre la puerta a la obtención de los factores $k_{\epsilon}$ y $\rho_{s}^{*}$ en la fase gaseosa y su posible extensión para el cálculo de propiedades termodinámicas del sistema para altas densidades. El entendimiento de tal efecto intenta dar así una explicación al mecanismo ejercido por la frustración y su responsabilidad en la sobreestimación del efecto dipolar sobre el equilibrio líquido-vapor de fluidos SM (así como de esferas duras dipolares) encontrado con el potencial de Keesom u otras interacciones promediadas angularmente $[9,31]$.

\subsubsection{Tercer coeficiente virial efectivo}

La propuesta matemática para la frustración dada por la ecuación 4.48 puede ahora ser incluida en el segundo coeficiente virial efectivo, ec. 4.43, obteniéndose la expresión modificada para este coeficiente

$$
\begin{aligned}
B_{\text {mod }}(T, \rho) & =\frac{2 \pi}{3} \delta^{3} \sum_{j=0}^{\infty}\left(-\frac{2^{j}}{4 j !}\right) \Gamma\left[\frac{2 j-1}{4}\right]\left(\frac{\epsilon}{k_{\mathrm{B}} T}\right)^{\frac{2 j+1}{4}}\left(1-\frac{2 j+1}{4} \gamma_{\epsilon} \rho^{*}\right) \\
& =B_{\mathrm{LJ}}(T)-\frac{\gamma_{\epsilon} \delta^{3}}{T} \frac{\partial B_{\mathrm{LJ}}(T)}{\partial(1 / T)} \rho
\end{aligned}
$$

donde, como paso intermedio, ha sido utilizada la forma del segundo coeficiente virial dada por la ecuación A.24 pág. 108. Los parámetros $\epsilon$ y $\delta$ son la energía y la posición del mínimo, respectivamente, de la función Lennard-Jones. Para trabajar con esta ecuación se escribe en unidades reducidas

$$
B_{\text {mod }}^{*}\left(T^{*}, \rho^{*}\right)=\frac{F_{\delta}^{3}}{\sqrt{2}} B_{\mathrm{LJ}}^{*}\left(\frac{T^{*}}{F_{\epsilon}}\right)-\frac{\gamma_{\epsilon} F_{\delta}^{6} \rho^{*}}{\sqrt{2} T^{*}}\left[\frac{\partial B_{\mathrm{LJ}}^{*}\left(\frac{T^{*}}{F_{\epsilon}}\right)}{\partial\left(1 / T^{*}\right)}\right]_{\epsilon_{\mathrm{ef}}, \delta_{\mathrm{ef}}}
$$

apareciendo los parámetros reducidos de energía y distancia, $F_{\epsilon}$ y $F_{\delta}$, y recalcando la constancia de los factores $\epsilon_{\text {ef }}$ y $\delta_{\text {ef }}$ en la derivada parcial. El primer término del lado derecho de esta expresión es precisamente el segundo coeficiente virial reducido dado por la ecuación 4.43 al cual se le resta un término proporcional a la densidad reducida.

Sustituyendo estos dos primeros coeficientes viriales efectivos, $B_{\mathrm{mod}}^{*}$ (primer orden en $\rho$ ), y a $C_{\mathrm{ef}}^{*}$ (segundo orden en $\rho$ ) en el desarrollo del virial, ec. (2.19), se obtiene la presión hasta precisamente el segundo orden en la densidad. Tomando en cuenta que la expresión efectiva para el segundo coeficiente virial de un fluido SMP genera buenos resultados, entonces es evidente que el término extra en la ecuación anterior debe anexarse a la ec (4.45) resultando en una nueva definición efectiva del tercer coeficiente virial para el fluido SMP. Esto se expresa matemáticamente de la siguiente forma

$$
C_{\mathrm{ef}}^{*}=\frac{F_{\delta}^{6}}{2}\left[C_{\mathrm{LJ}}^{*}\left(\frac{T^{*}}{F_{\epsilon}}\right)-\frac{\sqrt{2} \gamma_{\epsilon}}{T^{*}}\left[\frac{\partial B_{\mathrm{LJ}}^{*}\left(\frac{T^{*}}{F_{\epsilon}}\right)}{\partial\left(1 / T^{*}\right)}\right]_{\epsilon_{\mathrm{ef}}, \delta_{\mathrm{ef}}}\right]
$$


donde $B_{\mathrm{LJ}}^{*}$ y $C_{\mathrm{LJ}}^{*}$ están dados por las ecuaciones A.24 y A.37 respectivamente.

De la expresión anterior, $\gamma_{\epsilon}$ es el único parámetro desconocido y debido a que ésta nueva función caracteriza a la función $j_{\epsilon}$ su conocimiento a detalle es de importancia para la explicación del efecto de frustración.

Cuando se utiliza el caso SM es sencillo encontrar la forma exacta de $\gamma_{\epsilon}$. Si se supone que $C_{\mathrm{ef}}^{*}$ iguala al tercer coeficiente virial "verdadero" de un fluido SM $C_{\mathrm{SM}}[36]$, de tal manera que

$$
C_{\mathrm{ef}}^{*}\left(T^{*}, \mu^{*}, 0\right)=C_{\mathrm{SM}}^{*}\left(T^{*}, \mu^{*}\right)
$$

en donde se hace hincapié en que el lado izquierdo se evalúa para $\alpha^{*}=0$. A partir de esta igualdad y de la ec. (4.52) se resuelve directamente para $\gamma_{\epsilon}$ llegando finalmente a

$$
\gamma_{\epsilon}=\frac{T^{*}}{\sqrt{2}}\left[\frac{\partial B_{\mathrm{LJ}}^{*}\left(\frac{T^{*}}{F_{\epsilon}}\right)}{\partial\left(1 / T^{*}\right)}\right]_{\epsilon, \delta}^{-1}\left\{C_{\mathrm{LJ}}^{*}\left(\frac{T^{*}}{F_{\epsilon}}\right)-\frac{2}{F_{\delta}^{6}} C_{\mathrm{SM}}^{*}\left(T^{*}, \mu^{*}\right)\right\}
$$

con una dependencia clara con $\mu^{*}$ y $T^{*}$.

Debe recalcarse el hecho de que, aunque el factor $\gamma_{\epsilon}$ se extrajo usando tan solo información para el caso SM, puede ser usado para el caso polarizable. Esto se debe a que $\gamma_{\epsilon}$ es en sí mismo un factor de corrección y cualquier dependencia en $\alpha^{*}$ que tuviese, al ser incluida en la energía 4.46], generaría términos de orden mayor a uno en la polarizabilidad los que, como ya hemos planteado en un inicio, han sido despreciados.

\subsubsection{Presión virial efectiva SM}

Con expresiones explícitas para el segundo y tercer coeficientes viriales efectivos, la presión virial del fluido a densidades moderadas se define como

$$
\frac{P_{\mathrm{ef}}^{*}}{\rho^{*} T^{*}}=1+B_{\mathrm{ef}}^{*} \rho^{*}+C_{\mathrm{ef}}^{*} \rho^{* 2}
$$

donde $P_{\mathrm{ef}}^{*}=P_{\mathrm{ef}} \sigma^{3} / \epsilon$ y los coeficientes viriales efectivos $B_{\mathrm{ef}}^{*}$ y $C_{\mathrm{ef}}^{*}$ están caracterizados por las ecuaciones 4.43 . y 4.52 . La figura 4.11 muestra la presión $P_{\mathrm{SM}}^{*}$ del fluido $\mathrm{SM}$ en el caso que $\mu^{* 2}=3.0$ y $T^{*}=1.8$ (cercano a la temperatura crítica) en la región de bajas densidades y calculada para diferentes aproximaciones. En esta figura se incluyen dos curvas que representan las presiones efectivas obtenidas vía la ecuación (4.55); la primera considerando términos hasta segundo orden en $\rho^{*}$ y la segunda hasta tercer orden. Como referencia, se presenta la presión hasta el segundo $B_{\mathrm{SM}}^{*}$, y tercer orden $C_{\mathrm{SM}}^{*}$, en el desarrollo virial hallados en las referencias $[33,34,36]$ y a cuyas expresiones les llamaremos presiones viriales "exactas". También por comparación, la figura incluye la presión del fluido LJ calculada hasta tercer orden; esto es, cuando en la ecuación 4.55 se sustituye $\mu^{*}=0$.

Nótese de esta figura que la presión virial "exacta" del fluido SM es efectivamente más pequeña que la del caso no polar (el fluido LJ) lo cual significa que hasta tercer orden el efecto neto de las interacciones dipolares es aún atractiva. Entonces, comparando la presión virial "exacta" con la presión a segundo orden vemos que el efecto neto del tercer coeficiente virial del fluido SM es positivo; esto es, este coeficiente contribuye efectivamente con una repulsión neta. El modelo efectivo sin frustración da resultados muy cercanos al "exacto" calculado a segundo orden y por lo mismo subestimando la presión. Una vez que el tercer coeficiente virial efectivo es corregido al incluir el efecto de frustración, la presión concuerda con el resultado correcto mostrado por la línea negra sólida en la figura 4.11. Además, como se hará explícito en la siguiente sección, el modelo 4.41 con la frustración incluida puede ser usado para intentar representar de manera conveniente la ecuación de estado de un fluido SMP a temperaturas incluso mayores a la crítica.

En la misma gráfica se incluyen resultados de dinámica molecular de un fluido SM con momento dipolar $\mu^{* 2}=3.0$, a temperatura $T^{*}=1.8$, para todas las densidades allí mostradas. El primer conjunto de presiones pertenece a Bartke et. al. [33] y el segundo fue obtenido como parte de este trabajo [42]. En específico, nuestros datos de presión provienen de simular 500 dipolos puntuales durante 5000 colisiones posteriores al equilibro. Los 


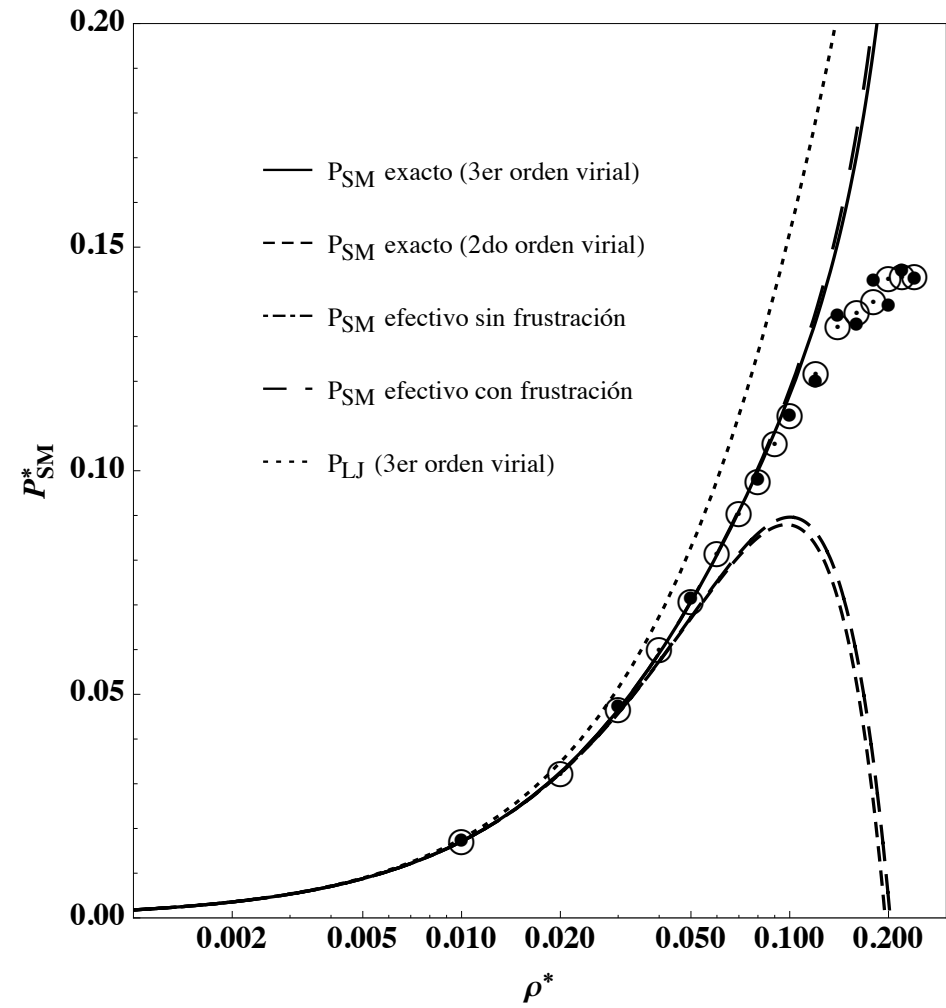

Figura 4.11: Presión vs densidad numérica $\left(P_{\mathrm{SM}}^{*} \quad\right.$ vs $\left.\rho^{*}\right): T^{*}=1.8, \mu^{* 2}=3.0 . \mathrm{En}$ esta figura se presentan las presiones explícitas SM correspondientes a la aproximación del virial hasta segundo y tercer orden. También se presentan las presiones SM efectivas, hasta tercer orden del virial, sin y con frustración. La serie virial hasta tercer orden coincide con los resultados de simulación de Bartke et. al. [33] $(\odot)$ y de este trabajo $(\bullet)$ hasta densidades de 0.1. Como referencia, se ha incluido a la presión del fluido LJ, $\left(\mu^{*}=0\right)$, hasta tercer orden del virial.

dipolos puntuales interaccionan mediante un potencial SM, ec. 2.16), por lo que en el código deben incluirse rutinas que incluyan la anisotropía del dipolo y su interacción de largo alcance. Esto significa que, a la par de la dinámica simple, deben incluirse el algoritmo Verlet dado por las ecuaciones 2.39 2.41) que involucran la rotación de las partículas junto con el método de sumas de Ewald (véase sección 2.6.2 que considera las interacciones dipolo-dipolo. Como puede observarse, por debajo de densidades reducidas de 0.1 la presión obtenida por simulación concuerda con la del desarrollo virial hasta el tercer orden en la densidad; a densidades más altas el cuarto coeficiente virial comienza a ejercer un efecto notable sobre la presión del fluido.

\subsection{Ecuación de estado de un fluido SMP}

Hasta ahora, se ha establecido que a bajas densidades, y momentos dipolares pequeños, el potencial esfericalizado $\Phi_{\mathrm{SMPs}}$ es conformal a la función LJ y su validez ha sido analizada, con buenos resultados, para el caso del segundo coeficiente virial. Cuando se intenta extender la validez de dicho modelo a densidades ligeramente mayores se ha requerido de un factor extra el cual incluye, de manera efectiva, la frustración energética entre un par de partículas dipolares cuando se encuentra presente otra del mismo tipo. En vista de ello, en la sección anterior se ha realizado una propuesta matemática con la ec. 4.49), basada en los aspectos básicos de tal fenómeno, con el fin de explicarlo.

Dicha propuesta, aplicada en el cálculo de $P_{\mathrm{ef}}^{*}$, ha mostrado que es suficiente trabajar con términos de hasta tercer orden en el desarrollo virial para lograr resultados adecuados; todo ello a densidades moderadas. Sin embargo, el planteamiento realizado sobre $j_{\epsilon}$ para sistemas alejados de la fase gaseosa abre la puerta para el estudio de tal fenómeno en sistemas más densos. Así, establecida la forma exacta de $\gamma_{\epsilon}$, mediante la ecuación 4.54, tan solo queda averiguar el comportamiento de los parámetros $k_{\epsilon} \mathrm{y} \rho_{s}^{*}$ que definen la forma general de $j_{\epsilon}$, ec. 4.49.

Con la presión efectiva calculada a nivel del virial, un paso consistente a dar es hallar la presión completa del sistema SMP dentro del marco de la conformalidad. Esto es, usando los parámetros efectivos $\epsilon_{\text {ef }}$ y $\delta_{\text {ef }}$, ecs. 
4.46 y 4.47), en una expresión conocida de la EDE del fluido LJ obtendremos de manera efectiva la respectiva EDE del fluido SMP.

Usando ahora el mismo desarrollo empleado con los coeficientes viriales, la presión total efectiva de un fluido SMP para densidades cercanas a la fase líquida es

$$
P_{\mathrm{ef}}^{*}\left(\rho^{*}, T^{*}\right)=\frac{\sqrt{2} F_{\epsilon} j_{\epsilon}}{F_{\delta}^{3}} P_{\mathrm{LJ}}^{*}\left(\frac{F_{\delta}^{3}}{\sqrt{2}} \rho^{*}, \frac{T^{*}}{F_{\epsilon} j_{\epsilon}}\right)
$$

en donde se ha usado explícitamente la definición de la frustración y el lado derecho corresponde a la forma analítica de la presión total de un fluido LJ dada por Johnson y cuyas características generales pueden hallarse en la referencia original [43]. Al escribir la EDE-LJ en términos de los parámetros moleculares efectivos se genera una EDE efectiva para sustancias SMP, por la cual a la expresión 4.56 se le ha denominado EDE-SMP. La dependencia de la presión SMP debida al momento dipolar y a la polarizabilidad es introducida vía las ecuaciones 4.46, 4.47, y el factor $j_{\epsilon}$.

La ecuación (4.56) para el sistema efectivo no estará determinada completamente debido a que los parámetros $k_{\epsilon} \mathrm{y} \rho_{s}^{*}$ declarados en la expresión (4.49) no son conocidos. Sin embargo, la ecuación (4.54) junto con $\gamma_{\epsilon}=k_{\epsilon} / \rho_{s}^{*}$, reduce el problema a hallar sólo uno de los parámetros requeridos. Datos de simulación de este tipo de sistemas hecho por diversos autores son una fuente importante de información que se usará en este trabajo para hallar así la forma de la función $j_{\epsilon}$.

Como puede observarse a partir de la ecuaciones (4.48) hasta (4.54), el efecto de frustración sólo tiene una dependencia directa con el momento dipolar; sin embargo como ya se ha mencionado antes, su construcción permite emplearlo en el caso de sistemas polarizables. De esta forma la presión $P_{\text {ef }}^{*}$ dada por 4.56 puede ser ocupada directamente para el pronóstico de propiedades termodinámicas en sistemas SMP. Resultados tales como la temperatura crítica y el equilibrio líquido-vapor para fluidos SM se obtendrán usando 4.56 en la parte final de este capítulo y serán comparados con datos de simulación también reportados por distintas fuentes para fluidos SMP.

\subsubsection{Metodología}

Usando a $\rho_{s}^{*}$ como parámetro libre, el perfil de la isoterma $P_{\mathrm{ef}}^{*}\left(\rho^{*}, T^{*}\right.$ constante $)$ puede modificarse de tal manera que, para un sistema dado, se haga coincidir (dentro del error de simulación) con resultados obtenidos vía simulación; en específico, se usan las densidades de coexistencia en el gas y en el líquido $\left(\rho_{v}^{*}\right.$ y $\rho_{l}^{*}$, respectivamente), para $\mu^{*}$ y $T^{*}$ dados. Así, con la densidad de coexistencia en el gas, $\rho_{v}^{*}$, se obtiene la presión $P_{\mathrm{ef}}^{*}$, vía la ec. 4.55, y con el fin de cumplir el equilibrio termodinámico, esta presión debe ser igual a la de la fase líquida. Con estos dos datos, $\left(P_{\mathrm{ef}}^{*}, \rho_{l}^{*}\right)$, la ecuación 4.56 puede resolverse numéricamente para obtener la densidad $\rho_{s}^{*}$ adecuada para este estado.

Debido a que el modelo efectivo LJ, ec. [4.41, es aproximado para $\mu^{* 2} \leq 4.0$ y densidades moderadas solo se usarán datos de simulación dentro de este régimen de intensidades del dipolo. Resultados de Smit et. al. [44] y van Leeuwen et. al., [45] obtenidos con simulación GEMC, para temperaturas menores a la crítica, son utilizados principalmente debido a su regularidad, al número extenso de datos y a que han sido obtenidos para momentos dipolares menores al del límite aquí planteado; otros conjuntos de datos obtenidos de diversas investigaciones sobre el tema servirán como apoyo a los resultados finales.

Un ejemplo del procedimiento antes descrito se muestra en la figura 4.12 para un sistema SM caracterizado por $\mu^{* 2}=1.0$ y $T^{*}=1.2$. En ella se muestran las isotermas generadas por la ecuación 4.56 sin incluir el efecto de frustración $j_{\epsilon}=1$, e incluyéndolo $j_{\epsilon}<1$, y son comparadas con los datos de simulación correspondientes a este mismo estado [45]. De la figuras puede observarse que a bajas densidades las dos curvas coinciden con el punto de simulación y cuando la densidad aumenta comienzan a separarse. En la zona líquida, cuando no se incluye la frustración, la isoterma generada queda muy por debajo del punto simulado y sólo cuando se incluye una frustración de $j_{\epsilon}=0.932$ la isoterma concuerda con los puntos de simulación. 


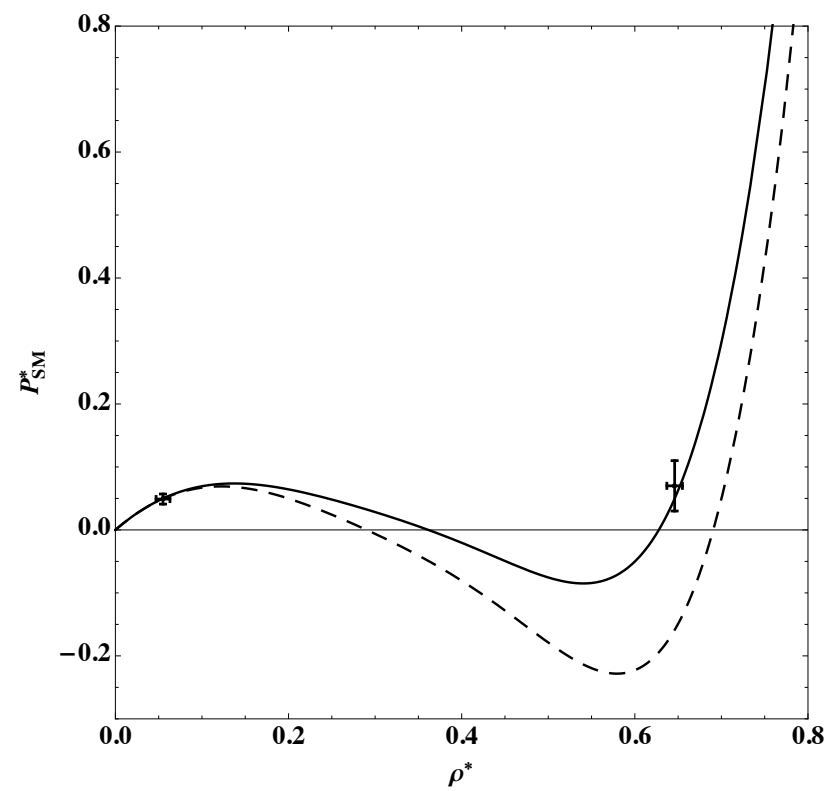

Figura 4.12: Método de obtención de $\rho_{s}^{*}$ para un sistema $\mathrm{SM}, \mu^{* 2}=1.0$ y $T^{*}=1.2$. La curva a trazos muestra la isoterma obtenida sin el efecto de frustración. Cuando el efecto es incluido (curva sólida), $\rho_{s}^{*}=0.292$, la isoterma formada concuerda con los puntos de simulación.

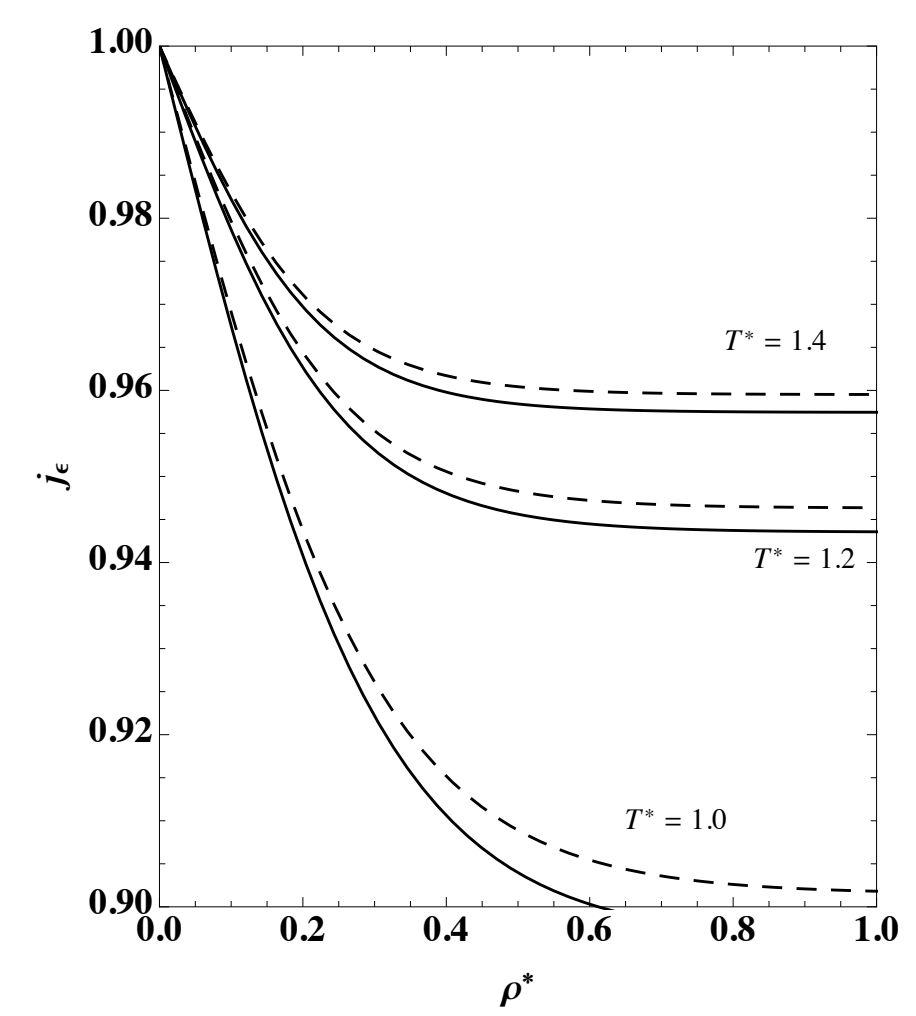

Figura 4.13: Efecto de frustración y su dependencia con la densidad. Sistema SM, $\mu^{* 2}=$ 1.0 (líneas sólidas) y $\mu^{* 2}=0.98$ (líneas a trazos) para tres distintas temperaturas. 
El proceso anterior es repetido para el resto de los datos utilizados obteniendo para cada uno de ellos un valor para la densidad de saturación $\rho_{s}^{*}$. Todo este conjunto de datos (ver tabla 4.3) tiene una dependencia con la temperatura y el momento dipolar y han sido parametrizados de la siguiente forma

$$
\rho_{s}^{*}\left(\mu^{*}, T^{*}\right)=\frac{0.218489+0.118729 \mu^{* 2}-0.0129663 \mu^{* 4}}{T^{*}}+\frac{-0.0168 \mu^{* 8}+0.00129676 \mu^{* 12}}{T^{* 3}}
$$

cuya validez se limita a los momentos dipolares manejados y las respectivas temperaturas subcríticas.

\begin{tabular}{|c|c|c|c||c|c|c|c||c|c|c|c|}
\hline \multicolumn{5}{|c||}{$\mu^{* 2}=1.0$} & \multicolumn{5}{c||}{$\mu^{* 2}=2.0$} & \multicolumn{5}{c|}{$\mu^{* 2}=3.0$} \\
\hline$T^{*}$ & $\rho_{s}^{*}$ & $T^{*}$ & $\rho_{s}^{*}$ & $T^{*}$ & $\rho_{s}^{*}$ & $T^{*}$ & $\rho_{s}^{*}$ & $T^{*}$ & $\rho_{s}^{*}$ & $T^{*}$ & $\rho_{s}^{*}$ \\
\hline 1.0 & 0.3167 & 1.34 & 0.2366 & 1.15 & 0.2200 & 1.50 & 0.2155 & 1.40 & 0.1731 & 1.70 & 0.1844 \\
1.05 & 0.2954 & 1.35 & 0.2296 & 1.20 & 0.2276 & 1.52 & 0.2105 & 1.45 & 0.1791 & 1.72 & 0.1827 \\
1.10 & 0.2792 & 1.36 & 0.2249 & 1.25 & 0.2277 & 1.54 & 0.2099 & 1.50 & 0.1833 & 1.74 & 0.1822 \\
1.15 & 0.2855 & 1.37 & 0.2109 & 1.30 & 0.2304 & 1.55 & 0.2100 & 1.55 & 0.1861 & 1.75 & 0.1827 \\
1.20 & 0.2921 & 1.38 & 0.2263 & 1.35 & 0.2321 & 1.56 & 0.2115 & 1.60 & 0.1871 & 1.76 & 0.1834 \\
1.25 & 0.2762 & 1.39 & 0.2224 & 1.40 & 0.2283 & 1.58 & 0.2236 & 1.62 & 0.1873 & 1.775 & 0.1871 \\
1.30 & 0.2520 & 1.40 & 0.2285 & 1.45 & 0.2234 & 1.59 & 0.2828 & 1.65 & 0.1859 & 1.80 & 0.2109 \\
1.32 & 0.2407 & 1.41 & 0.2300 & 1.47 & 0.2206 & 1.60 & 0.1844 & 1.68 & 0.1852 & 1.82 & 0.1831 \\
\hline
\end{tabular}

Tabla 4.3: Datos de densidades de saturación obtenidas mediante el método dado en esta sección. Los datos de simulación usados para su obtención provienen de los trabajos de Smit et. al. [44] y van Leeuwen et. al. [45]. Las densidades $\rho_{l}^{*}$ y $\rho_{v}^{*}$ empleadas pueden revisarse en dichas referencias.

El cambio de $j_{\epsilon}$ con respecto a la densidad se observa en la figura 4.13 para dos momentos dipolares y distintas temperaturas. Como se muestra en la figura, a bajas densidades $\gamma_{\epsilon}$ es el factor dominante, ver ec. 4.48), y se modifica rápidamente a bajas temperaturas. Para densidades mayores $j_{\epsilon}$ tiende al valor $1-k_{\epsilon}$ (o bien $\left.1-\gamma_{\epsilon} \rho_{s}^{*}\right)$, mientras que $\rho_{s}^{*}$ indica la densidad a la cual se inicia dicha tendencia. En este caso se observa que dicho factor puede reducir la energía atractiva efectiva hasta un $10 \%$, a altas densidades. Comportamientos semejantes se observan cuando la intensidad del dipolo aumenta y la temperatura se mantiene fija.

Antes de retomar la ec. 4.57) para los sistemas SM a altas densidades y posteriormente aplicarla a sistemas SMP, es necesario realizar algunas observaciones importantes. Es claro que en esta última parte del trabajo no se ha producido nueva información termodinámica. Para un fluido SM a densidad cercana al líquido se ha rescatado la información disponible para construir una función LJ, que con parámetros convenientes, sustituye el potencial SM (ángulo-dependiente) por otro potencial que es esférico.

\subsubsection{Temperatura crítica}

El punto crítico del fluido SM se calcula de forma numérica resolviendo simultáneamente las ecuaciones 2.2 y 2.3 , pág. 6. Se utiliza la presión total reducida del fluido SM, $P_{\text {ef }}^{*}=P_{\text {ef }} \sigma^{3} / \epsilon$, sustituyendo $\alpha^{*}=0$ en la ecuación 4.56), y calculando las dos derivadas con respecto a la densidad $\rho^{*}=\rho \sigma^{3}$.

La figura 4.14 muestra el comportamiento de $T_{\mathrm{c}}^{*}$ como función de $\mu^{* 2}$ para un fluido SM; los resultados de este trabajo son comparados con resultados de simulación [33,44-47]. Como se observa en la figura, el potencial efectivo primitivo (aquel que no incluye el efecto de frustración) reproduce cualitativamente la tendencia real de la temperatura crítica respecto a $\mu^{* 2}$; sin embargo, sobreestima el efecto de los dipolos. Cuando la función 


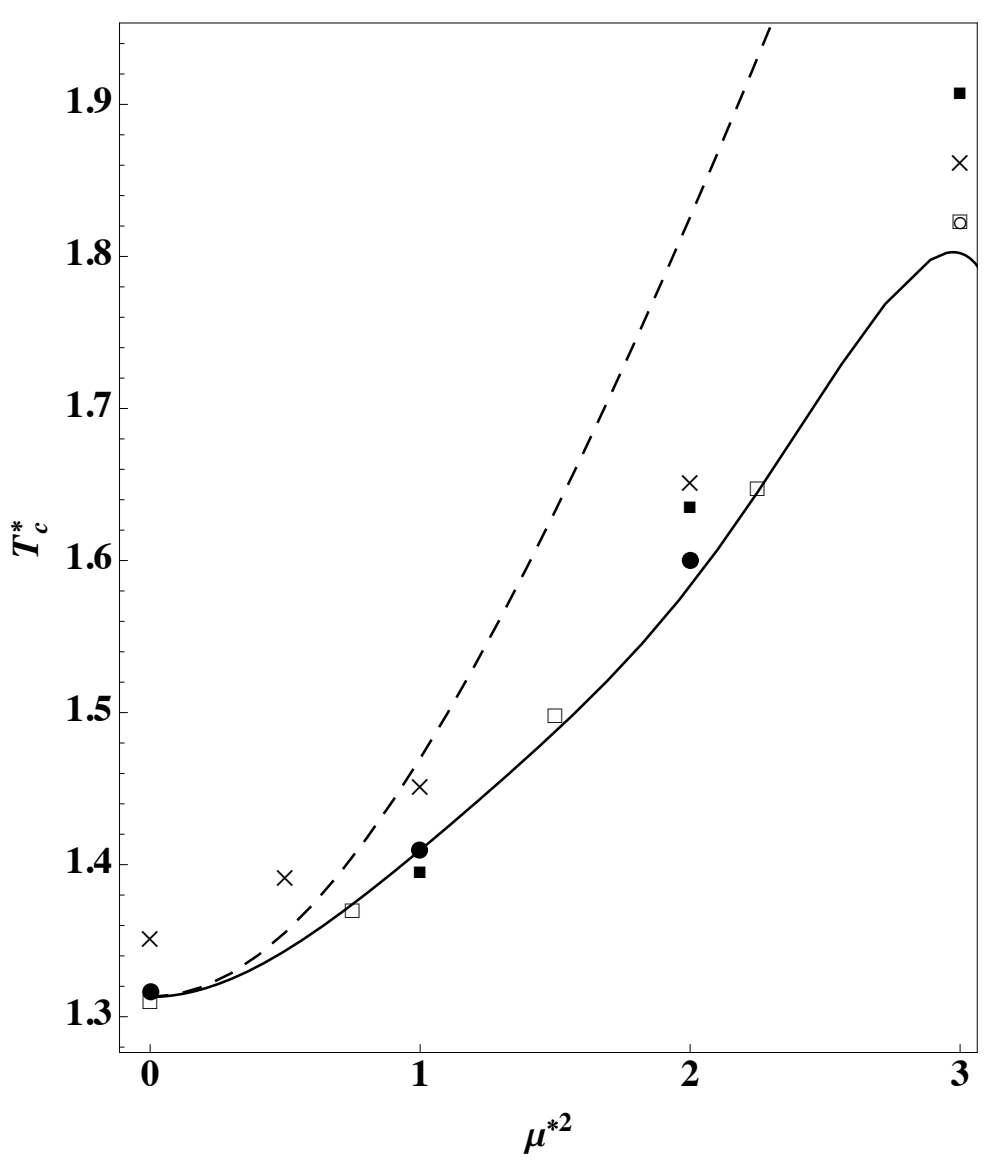

Figura 4.14: Temperatura crítica para el fluido SM como una función de la intensidad dipolar $\mu^{*}$. La predicción con el potencial efectivo primitivo, ec. 4.32, (línea a trazos) se compara con los resultados de simulación: Smit

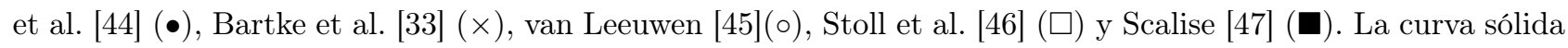
se obtiene cuando se incluye el factor de frustración en $\epsilon$ debido al factor $j_{\epsilon}$, como se discute en el texto. 
$\epsilon_{\mathrm{ef}}$ incluye los efectos de dipolos extras, la temperatura crítica $T_{\mathrm{c}}^{*}$ crece acorde los resultados de simulación con la tendencia correcta hasta intensidades cercanas a $\mu^{* 2}=3.0$.

\subsubsection{Coexistencia líquido-vapor para fluidos SMP}

En esta sección se presentan las curvas de coexistencia de los fluidos SMP obtenidas mediante la regla de áreas iguales de Maxwell $[13,48]$. La coexistencia de fase, a una temperatura dada $T^{*}$, requiere se cumplan simultáneamente las siguientes ecuaciones

$$
P_{\mathrm{ef}}^{*}\left(\rho_{v}^{*}, T^{*}\right)=P_{\mathrm{ef}}^{*}\left(\rho_{l}^{*}, T^{*}\right)
$$

y

$$
\nu^{*}\left(\rho_{v}^{*}, T^{*}\right)=\nu^{*}\left(\rho_{l}^{*}, T^{*}\right),
$$

para ambas fases, con $P_{\mathrm{ef}}^{*}$ dada por la ec. $4.56, \nu^{*}=\nu / k_{\mathrm{B}} T$ es el potencial químico $\nu$ reducido, y $\rho_{l}^{*}$ y $\rho_{v}^{*}$ son las densidades del líquido y del vapor del fluido, respectivamente. La ecuación (4.59) corresponde a una energía libre de Gibbs constante $(d G=0)$, por lo que puede demostrarse que el sistema de ecuaciones a partir del cual se calcula numéricamente la presión de equilibrio $P_{s}^{*}$ y las densidades de coexistencia, es [15]

$$
\begin{aligned}
P_{s}^{*} & =P_{\mathrm{ef}}^{*}\left(\rho_{v}^{*}, T^{*}\right)=P_{\mathrm{ef}}^{*}\left(\rho_{l}^{*}, T^{*}\right) \\
\int_{\rho_{v}^{*}}^{\rho_{l}^{*}} \frac{P_{\mathrm{ef}}^{*}\left(\rho^{*}, T^{*}\right)}{\rho^{* 2}} d \rho^{*} & =P_{s}^{*}\left(\frac{1}{\rho_{l}^{*}}-\frac{1}{\rho_{v}^{*}}\right) .
\end{aligned}
$$

Figura 4.15: Curvas ortobáricas para un fluido SM con $\mu^{* 2}=1.0$ obtenidas con el modelo de potencial efectivo: sin (curva a trazos) y con frustración (curva sólida). Los símbolos son datos de simulación obtenidos por Smit et. al. [44] (o) y Kiyohara et. al. [49] (ם). La curva restante (inferior) corresponde a la coexistencia del fluido Lennard-Jones. Los puntos críticos de los tres casos están representados por el símbolo $(\mathbf{\Lambda})$.

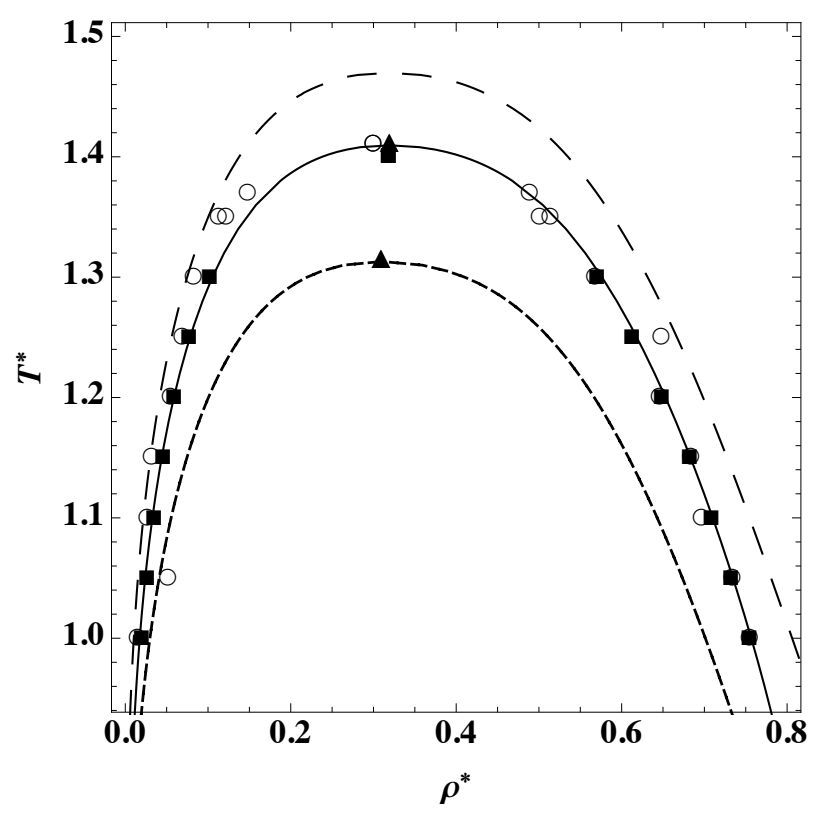

En la figura 4.15 se presentan las curvas LV calculadas con este método junto con los resultados de simulación de Smit et. al. [44] y Kiyohara et. al. [49]. La figura también incluye los resultados de distintas aproximaciones con este trabajo para $\mu^{* 2}=1.0$, obtenida con el potencial efectivo primitivo $\left(j_{\epsilon}=1\right)$ y con el factor de frustación incluido $\left(0 \leq j_{\epsilon} \leq 1\right)$; como referencia, se ha incluido la curva de coexistencia para el fluido LJ. Al introducir los efectos dipolares a nivel de dos cuerpos (sin factor de frustración) se obtiene una curva de coexistencia $T^{*}-\rho^{*}$ por arriba de las respectivas para el potencial LJ y del fluido SM. Cuando la frustración se incluye vía el factor $j_{\epsilon}$, la EDE genera una curva ortobárica que desciende con respecto a la original concordando muy bien con los datos de Smit y de Kiyohara.

Las curvas de coexistencia para fluidos SM para distintos momentos dipolares en el rango $0.0 \leq \mu^{* 2} \leq 3.0$ son presentadas en la figura 4.16 junto con resultados de simulación de Smit et. al. [44], Kiyohara et. al. [49] y 


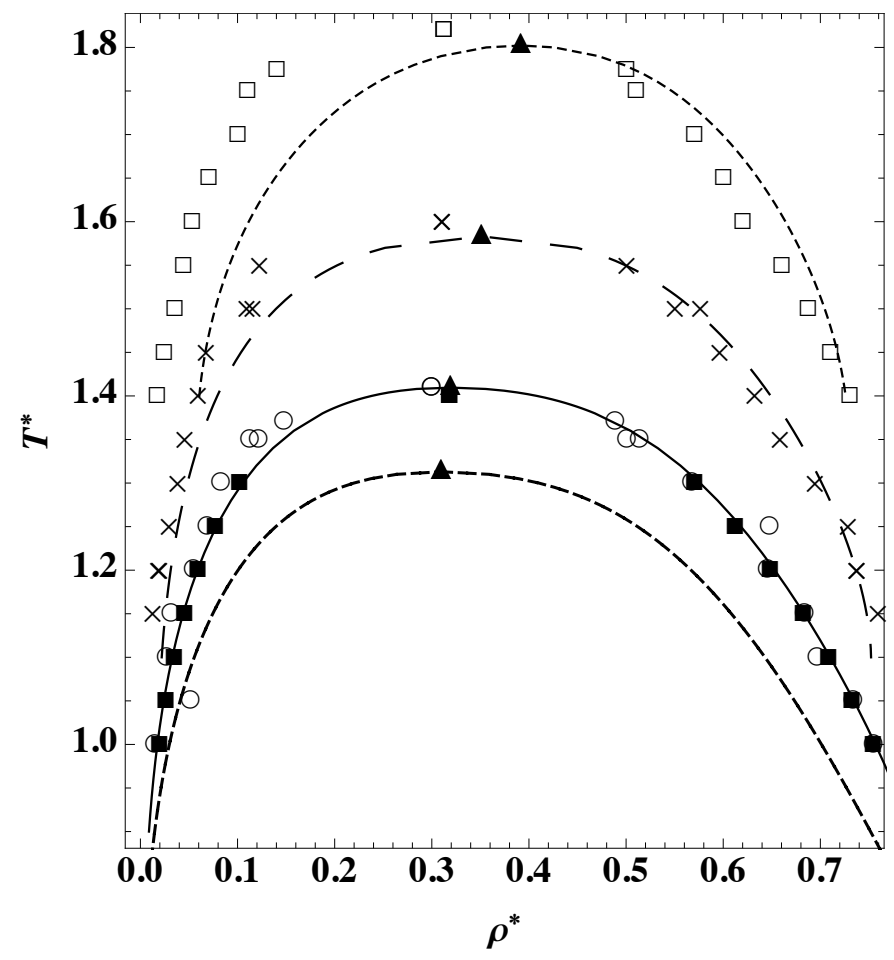

Figura 4.16: Curvas LV de fluido SM, para distintos momentos dipolares, obtenidas vía la EDE-SMP: De abajo hacia arriba: $\mu^{* 2}=$ $0.0,1.0,2.0$ y 3.0 donde sus respectivos puntos críticos están representados por el símbolo $\boldsymbol{\Lambda}$. Los símbolos restantes provienen de resultados de simulación [44, 45, 49].

Figura 4.17: Ortobáricas para un fluido SMP obtenidas con el modelo de potencial efectivo con efecto de frustración incluido y $\mu^{* 2}=$ 1.0. De abajo hacia arriba: $\alpha^{*}=0.0,0.03$, y 0.06. Los símbolos ( $\mathbf{\Delta})$ son sus puntos críticos. El resto de los símbolos son datos de simulación obtenidos por Kiyohara et. al. [49].

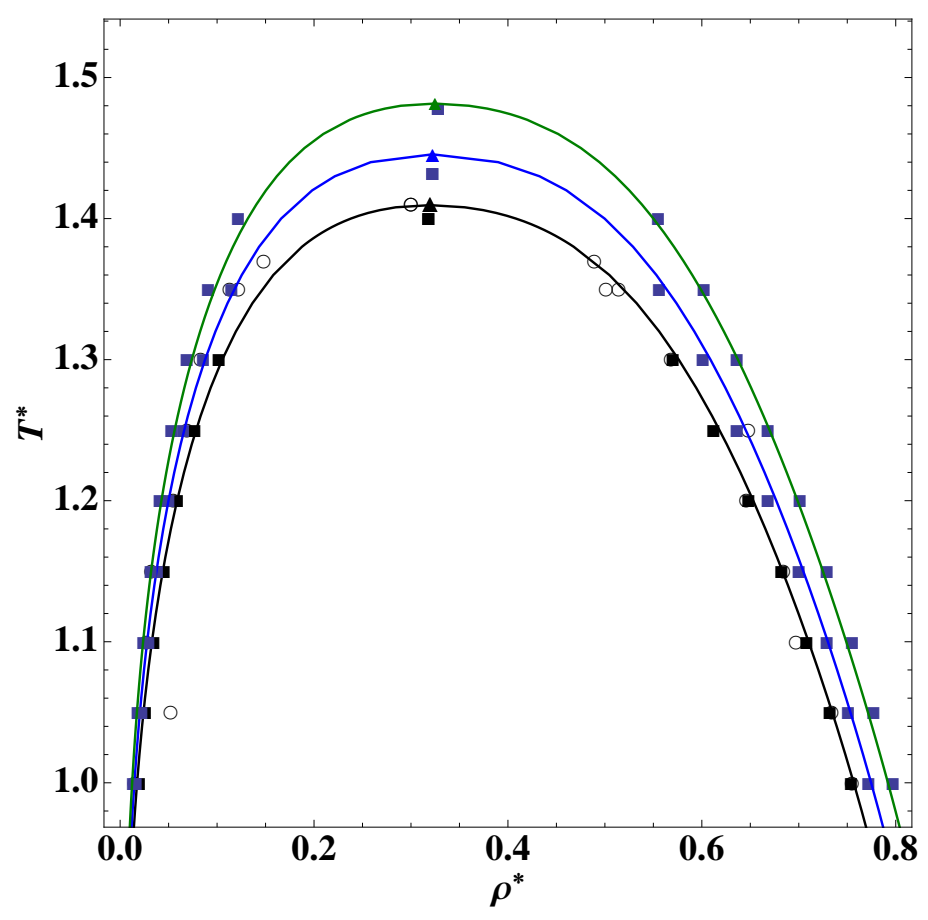


van Leuween [45]. Podemos ver que para $\mu^{* 2}=2.0$ la EDE efectiva es capaz de reproducir adecuadamente las densidades simuladas en la zona del líquido.

Debido a que el efecto de frustración no incluye dependencia con la polarizabilidad el mismo potencial efectivo usado en los casos anteriores puede ser usado para corroborar el modelo. Así, nuestro modelo puede incorporar los efectos de polarizabilidad a través de las ecs. 4.46) y 4.47). La figura 4.17 muestra las curvas ortobáricas para un sistema SMP: $\mu^{* 2}=1.0$ y distintas polarizabilidades. Todas las curvas presentan excelente concordancia con la simulación para una polarizabilidad de 0.06 [49].

\subsection{Simulación de sistemas SMP efectivos}

Puede probarse que el potencial efectivo $\Phi_{\text {ef }}$, construido en este capítulo, tiene sentido a nivel de estructura del líquido. Dicha prueba se ha realizado calculando la función de distribución radial de los sistemas efectivo y SM mediante simulaciones de la DM de los fluidos respectivos. Para el fluido SM, el código de DM aplica la técnica usual de las sumas de Ewald - ver sección 2.6.2 - y la resolución de las ecuaciones de la dinámica rotacional, ambas con el conjunto de subrutinas extras que implica su funcionamiento. En el caso del fluido efectivo (un fluido LJ) se utiliza una DM simple lo que intenta ser un ahorro computacional y temporal, con respecto a la DM anterior, para reproducir al sistema original SM.

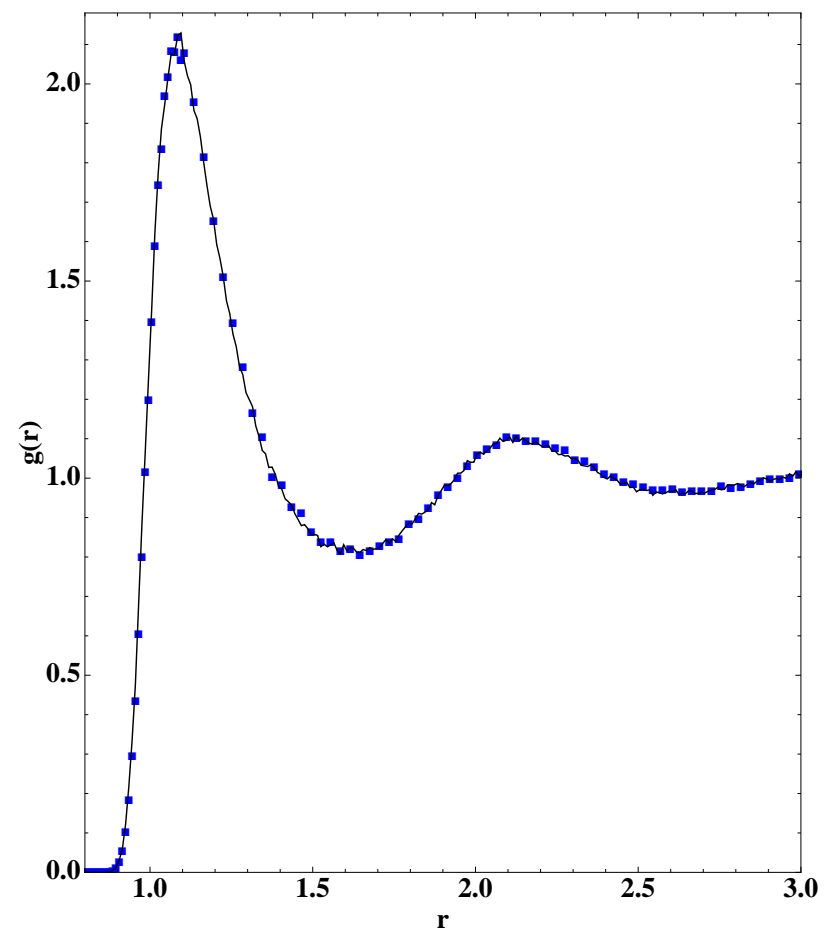

Figura 4.18: Función de distribución radial calculada vía dinámica molecular para un sistema SM y su equivalente efectivo LJ para $\mu^{* 2}=1.0$, $T^{*}=1.35$ y $\rho^{*}=0.6$. Los puntos pertenecen al fluido SM y la línea negra continua (interpolación de los datos de simulación) al LJ efectivo. Los parámetros efectivos de este último son obtenidos de las ecuaciones 4.46 and 4.47.

Todas las simulaciones fueron desarrolladas en la región del líquido, $\rho^{*}=0.6$ a una temperatura subcrítica $T^{*}=1.35 \mathrm{y}$ una intensidad dipolar $\mu^{*}=1.0$. La presión y la función de distribución radial $g(r)$, respectivas fueron calculadas en el ensamble NVT con $N=500$ partículas; se usaron 10000 colisiones para equilibrar los sistemas y 20000 colisiones adicionales para obtener promedios; el potencial LJ fue truncado a $r=3.5 \sigma$. El tiempo de simulación en el caso del sistema LJ efectivo fue aproximadamente 30 veces menor que el de la simulación SM.

Los parámetros efectivos usados en la simulación del fluido SMP efectivo fueron obtenidos directamente de las ecuaciones 4.46 y 4.47). En el estado de simulación arriba presentado se trabaja con los siguientes valores numéricos: $F_{\epsilon}=1.13$ y $F_{\delta}=1.11$, obtenidos directamente de las funciones 4.23 y 4.24 con parámetros $\mu^{*}$ y $T^{*}$ ya definidos arriba. En el equilibrio, el sistema LJ puro alcanza una presión de $P^{*}=0.46905$, mientras que 
el fluido SM alcanza una presión más pequeña $P^{*}=0.31734$; esta disminución en la presión se debe (como ya se ha mencionado anteriormente) a la fuerte atracción que surge de la interacción dipolo-dipolo. Por otro lado, en el equilibrio en el fluido LJ efectivo se alcanza una presión mucho menor que la del LJ estándar y mucho más cercana a la del caso SM: $P_{\mathrm{ef}}^{*}=0.31096$.

En cuanto a las correlaciones de estructura presentes, la función de distribución radial $g(r)$ para ambos sistemas es la cantidad que podemos cotejar directamente. Las funciones de distribución radial obtenidas mediante las simulaciones son mostradas en la figura 4.18, Los puntos que ahí aparecen corresponden a la simulación del sistema original SM y la línea sólida corresponde a la simulación del SM efectivo; es decir, del potencial LJ con parámetros moleculares dados por las ecuaciones 4.46 y 4.47). La concordancia entre las $g(r)$ del fluido SM y el fluido efectivo LJ son evidentes en esta figura.

\subsection{Conclusiones}

En este capítulo se ha obtenido un potencial esférico binario efectivo para fluidos cuyas partículas contienen un dipolo puntual y están sujetas a la polarización entre ellas. La interacción resultante es un potencial tipo ANC cuyos parámetros de energía $\epsilon_{\mathrm{ef}}$, de distancia $\delta_{\mathrm{ef}}$, y de forma $S_{\mathrm{R}}$ y $S_{\mathrm{A}}$ dependen (además de las variables de estado) de las características electrostáticas del sistema; de esta forma, el modelo efectivo logra incorporar en expresiones sencillas las interacciones de largo alcance presentes en sistemas de este tipo.

En la construcción del modelo se ha recurrido al principio de estados correspondientes y a la conformalidad entre los perfiles del potencial del sistema de estudio con respecto a uno de referencia: dos potenciales conformales comparten la misma termodinámica. Sin embargo, la dependencia orientacional del potencial hace difícil la aplicación de dicho principio por lo que se ha construido, a partir de dicha interacción, un potencial mucho más sencillo e independiente de la orientación relativa entre moléculas. Este potencial esférico (SMPs) se obtiene a partir del promedio configuracional del factor de Boltzmann en el ensamble canónico, ec. 4.20, y busca representar al sistema SMP de una manera mucho más simple. Así, se ha construido un potencial ANC conformal al potencial SMPs, pero que en la práctica reproduce la termodinámica del sistema SMP a bajas densidades, generando buenas expectativas para densidades más altas.

El primer paso de este trabajo consistió en esfericalizar el potencial SMP y comparar los perfiles de los potenciales resultantes SMPs con las funciones LJ y ANC, estableciendo así la conformalidad entre dichos potenciales. Así, para intensidades dipolares pequeñas $\left(\mu^{* 2} \leq 1.0\right)$ los potenciales SMPs de la gran mayoría de estados aquí manejados presentan conformalidad con respecto al fluido LJ pero a medida que el momento dipolar aumenta el perfil de ambos potenciales no coincide por lo que el PEC no se cumple. Este efecto se hace más evidente cuando se trabaja a bajas temperaturas. La principal desventaja de este método radica en que las comparaciones entre los perfiles se realizan cualitativamente y es aquí donde la teoría ANC muestra una mayor versatilidad: primero, pueden trabajarse sistemas SMP con intensidades polares mayores y, segundo, aportando un método cuantitativo con el cual se establece la existencia de conformalidad entre dos sistemas. En específico, bajo las condiciones $T^{*} \lesssim 2.0, \mathrm{y} / \mathrm{o} \mu^{* 2} \gtrsim 1.0$, es preferible un sistema efectivo tipo ANC con suavidades distintas a la unidad; esto significa que en el estudio de este tipo de sistemas polares un sistema LJ sólo será adecuado para momentos dipolares pequeños.

La conformalidad entre los potenciales ANC y el SMP esfericalizado permite calcular el segundo coeficiente virial del fluido SMP. Para esto, se emplean los parámetros efectivos ANC en la expresión analítica del segundo coeficiente virial ANC (ya conocido) obteniéndose una expresión analítica efectiva de este coeficiente para un fluido SMP. En la práctica, se sustituyen los parámetros moleculares ANC por los efectivos correspondientes a las ecuaciones 4.33 y 4.34, incluyendo las suavidades obtenidas por el método mostrado en la sección 4.4.6. Para poder compararlo con el fluido SMP, se reduce la expresión resultante en el paso anterior con los parámetros del kernel. El modelo efectivo es capaz de predecir el segundo coeficiente virial $B(T)$ de moléculas SM y su variante que incluye la polarizabilidad con excelentes resultados - ver figuras 4.8(a) para el caso SM y 
4.8(b) para el SMP (página 61) - incluso para momentos dipolares mayores a $\mu^{* 2}=4.0$, y con polarizabilidades también altas $\alpha^{*}=0.1$. Como ya se ha mencionado antes, cuando se trabaja con intensidades pequeñas del dipolo, $\mu^{* 2} \leq 1.0$, una función efectiva del tipo LJ es suficiente para reproducir el sistema gaseoso SM; este es una situación especial del caso ANC.

Si el procedimiento anterior se emplea para calcular el tercer coeficiente virial de un fluido SMP los resultados obtenidos no son los esperados; ni siquiera desde un punto de vista cualitativo. Esta contrariedad surge de usar un potencial de interacción binario en un coeficiente donde la presencia de un tercer cuerpo se hace importante, incluso a bajas densidades, debido a la interacción de largo alcance entre los dipolos. Para estudiar ese efecto se ha introducido el concepto de frustración $\left(j_{\epsilon}\right)$ el cual aparece cuando la configuración orientacional de mínima energía entre dos dipolos es perturbada por la presencia de una tercer molécula SMP; esa molécula extra "frustra" la configuración entre dos dipolos.

En este trabajo se ha hecho una propuesta matemática que incorpora al modelo efectivo el impacto de la frustración a nivel energético, haciendo compatible el cálculo exacto del tercer coeficiente virial para fluidos polares. A nivel del gas dicha propuesta tiene una dependencia lineal con la densidad y está dada por la ecuación 4.48. Si se considera que el potencial efectivo, con efecto frustrante incluido, reproduce exactamente al tercer coeficiente virial del sistema SM se puede obtener una expresión exacta para $j_{\epsilon}$. Si bien es cierto, que el modelo propuesto para la frustración no reproduce el tercer coeficiente virial de un fluido SMP (en el sentido de que no se está aportando teoría nueva en lo que respecta a esta propiedad del gas), lo que se logra es conocer la forma en que este nuevo factor influye en este tipo de fluidos a densidades a las que las interacciones electrostáticas ternarias comienzan a influir debido a su alcance.

Para densidades mayores, se ha propuesto un $j_{\epsilon}$ en el que el aumento de dipolos produce una saturación electrostática evitando así que la frustración continúe aumentando con la densidad. Este nuevo modelo, dado por (4.49), permite usar dicho concepto hasta densidades tales como la crítica e incluso mayores al considerar viable la aplicación de la teoría de estados correspondientes entre sistemas SMP y LJ; de esta forma la EDE de un fluido LJ (conocida de antemano) es ocupada para generar la correspondiente EDE de un sistema polar.

Con la información ganada al obtener la primera versión de $j_{\epsilon}$ y con la información del equilibrio líquidovapor de un fluido SM, se ha construido (en un proceso de retroalimentación) el nuevo modelo para 4.49. Provenientes de distintas fuentes, los datos de simulación del equilibrio LV en diversos estados de sistemas SM y SMP permiten obtener un serie de datos de densidades de saturación $\rho_{s}^{*}$ para distintos estados los cuales son parametrizados en función de las cantidades reducidas de la temperatura y el momento dipolar, obteniéndose así una expresión completa de $j_{\epsilon}$. A partir de este efecto se establece la EDE del fluido efectivo SMP (EDESMP). Como ya se ha mencionado, la polarizabilidad no aparece explícitamente en $j_{\epsilon}$ debido a que solo se han considerado términos de primer orden en $\alpha$. Sin embargo, esta misma ecuación puede usarse para sistemas polarizables. En principio, la validez del PEC solo se da en aquellos estados conformales de las funciones LJ y SMP por lo que los resultados aquí obtenidos sólo son aplicables para $\mu^{* 2} \leq 1.0$; para intensidades dipolares mayores los resultados se consideran simplemente como una aproximación.

La EDE-SMP con el factor de frustración incluido reproduce adecuadamente, como era de esperarse, la temperatura crítica y las curvas ortobáricas SMP para aquellos momentos dipolares en los que la conformalidad del sistema SMP con el LJ se hace presente. En aquellos estados en los que la conformalidad no es exacta y que se hallan limitados por los intervalos: $1.0 \leq \mu^{* 2} \leq 2.0$ y $\alpha^{*} \leq 0.06$, el nuevo modelo efectivo entrega resultados que concuerdan muy bien con aquellos que provienen de la simulación del equilibrio líquido-vapor de estos sistemas polares, - ver figura 4.14. Resulta claro de la figura 4.15 que cuando no se incluye $j_{\epsilon}$ en el término energético se obtienen ortobáricas que sobreestiman a las simuladas por otros autores. Esto puede deberse a que durante el promediado de la interacción SMP el factor frustrante existente en las interacciones de largo alcance es eliminado. La figura 4.16 muestra el buen comportamiento que arroja el sistema efectivo para estados con distinto momento dipolar, aunque para dipolos con intensidades $\mu^{* 2} \geq 2.0$ el modelo no reproduce adecuadamente a las ortobáricas reportadas. Sin embargo, como puede observarse en la figura 4.17, para el caso 
$\mu^{* 2}=1.0$, el sistema efectivo incorpora de manera exitosa el efecto debido a la polarización molecular.

Finalmente, el sistema SM y su contraparte efectiva son estudiados desde un punto de vista estructural al calcularse (mediante la simulación de la dinámica molecular del gas) la función de distribución radial. El algoritmo utilizado en la dinámica molecular para un sistema SM efectivo es el mismo al de un sistema LJ (simple y esférico) con interacciones $\delta_{\text {ef }}$ y $\epsilon_{\text {ef }}$ provenientes de las relaciones efectivas (4.46) y (4.47) los cuales incluyen el efecto dipolar frustrado. Es decir, la simulación de este sistema es mucho más sencilla, y con un tiempo de cómputo mucho menor al compararla con el empleado con el código establecido para estudiar las interacciones de largo alcance y una dependencia angular representativas del potencial SM. Un ejemplo de la simulación de estos sistemas se observa en la figura 4.18 en donde se presenta la respectiva $g(r)$ con una intensidad del dipolo de $\mu^{*}=1.0$ obtenida para los fluidos de estudio y efectivo. En la figura se observa la capacidad del modelo efectivo al reproducir la $g(r)$ de manera muy cercana a la del fluido SM original.

\section{Referencias}

[1] J. M. Deutch. Ann. Rev. Phys. Chem., 24:301-323, 1973.

[2] M. S. Wertheim. Ann. Rev. Phys. Chem., 30:471-501, 1979.

[3] B. J. Alder and E. L. Pollock. Ann. Rev. Phys. Chem., 32:311-329, 1981.

[4] W. H. Stockmayer. J. Chem. Phys., 9:398-402, 1941.

[5] A. D. Buckingham and J. A. Pople. Trans. Far. Soc., 51:1173-1179, 1955.

[6] W. H. Keesom. Phys. Z, 22:129, 1921.

[7] D. Cook and J. S. Rowlinson. Proc. R. Soc. Lond. A, 219:405-418, 1953.

[8] Y. Singh and S. Singh. J. Phys. B: Atom. Molec. Phys., 5:2039-2050, 1972.

[9] C. E. Woodward and S. Nordholm. Mol. Phys., 52(4):973-986, 1984.

[10] J. E. Ramos, F. del Río, and I. A. McLure. Phys. Chem. Chem. Phys., 3:2634-2643, 2001.

[11] E. Ávalos, F. del Río, and S. Lago. J. Phys. Chem. B, 109:508-517, 2005.

[12] J. D. Jackson. Classical Electrodynamics. John Wiley and Sons, Inc, 1999.

[13] J. P. Hansen and I. R. McDonald. Theory of Simple Fluids. Academic Press, London, 1986.

[14] J. O. Hirschfelder, C. F. Curtiss, and R. F. Bird. Molecular Theory of gases and liquids. Wiley, 1954.

[15] J. Bartke. Computer simulation of the Stockmayer fluid. Ph. D. Thesis, Bergische Universität Wuppertal, Germany, 2008.

[16] H. Yu and W. F. van Gunsteren. Comp. Phys. Comm., 172:69-85, 2005.

[17] V. Magnasco, M. Battezzati, A. Rapallo, and C. Costa. Chem. Phys. Lett., 428:231-235, 2006.

[18] J. R. Johnson and P. T. Eubank. Ind. Eng. Chem. Fund., 12:156-165, 1973.

[19] J. N. Israelachvili. Intermolecular and surface forces. Academic Press, London, 1997.

[20] R. K. Pathria. Statistical Mechanics. BH, Oxford, 2001.

[21] M. Battezzati and V. Magnasco. J. Phys. A: Math. Gen., 37:9677-9684, 2004. 
[22] M. Battezzati and V. Magnasco. J. Phys. A: Math. Gen., 38:6715-6728, 2005.

[23] P. C. Abbott. J. Phys. A: Math. Theor., 40:8599-8600, 2007.

[24] I. S. Gradshteyn and I. M. Ryzhik. Table of Integrals, series, and products. Elsevier, 2007.

[25] J. S. Rowlinson. Mol. Phys., 1:414, 1958.

[26] P. K. Bhattacharyya, A. K. Ghosh, and A. K. Barua. J. Phys. B: At. Mol. Phys., 3:526-535, 1970.

[27] Y. Singh and S. Singh. J. Phys. B: Atom. Molec. Phys., 4:776-785, 1971.

[28] G. T. Gao, J. B. Woller, X. C. Zeng, and W. Wang. J. Phys.: Condens. Matter, 9:3349-3360, 1997.

[29] Y. Singh and O. Joshi. Pramana, 15(5):407-412, 1980.

[30] P. Pathak, O. Singh, and O. Joshi. Pramana, 16(6):481-485, 1981.

[31] R. J. Sadus. Mol. Phys., 87(5):979-990, 1996.

[32] R. J. Sadus. Mol. Phys., 89(4):1187-1194, 1996.

[33] J. Bartke and R. Hentschke. Phys. Rev. E, 75:061503, 2007.

[34] S. C. Saxena and K. M. Joshi. Phys. fluids, 5(10):1217-1222, 1962.

[35] C. Vega, C. McBride, and C. Menduiña. Phys. Chem. Chem. Phys., 4:3000-3007, 2002.

[36] J. S. Rowlinson. J. Chem. Phys., 19:7, 1951.

[37] S. W. De Leeuw, C. P. Williams, and B. Smit. Fluid. Phase Eq., 48:99-109, 1989.

[38] G. C. A. M. Mooij, S. W. De Leeuw, B. Smit, and C. P. Williams. J. Chem. Phys., 97(7):5113-5120, 1992.

[39] R. D. Kamien and J. V. Selinger. J. Phys.: Condens. Matter, 13:R1, 2001.

[40] P. B. Allen. J. Chem. Phys., 120(6):2951-2962, 2004.

[41] S. H. L. Klapp and G. N. Patey. J. Chem. Phys., 112(8):3832-3844, 2000.

[42] A. Rocha-Ichante, F. del Río, and E. Ávalos. J. Chem. Phys., 133, 2010.

[43] J. K. Johnson, J. A. Zollweg, and K. E. Gubbins. Mol. Phys., 78(3):591-618, 1993.

[44] B. Smit, C. P. Williams, E. M. Hendriks, and S. W. de Leeuw. Mol. Phys., 68(3):765-769, 1989.

[45] M. E. van Leeuwen, B. Smit, and E. M. Hendriks. Mol. Phys., 78(2):271-283, 1993.

[46] J. Stoll, J. Vrabec, and H. Hasse. Fluid Phase Equil., 209:29-53, 2003.

[47] O. H. Scalise. Fluid Phase Equil., 253:171-175, 2007.

[48] E. A. and Guggenheim. Thermodynamics. An Advanced Treatment for Chemists an Physicists. Elsevier Science Publishers B. V., Amsterdam, 1967.

[49] K. Kiyohara, K. E. Gubbins, and A. Z. Panagiotopoulos. J. Chem. Phys., 106(8):3338-3346, 1997. 


\section{Capítulo 5}

\section{Sistemas de moléculas convexas}

\subsection{Introducción}

Las interacciones moleculares no esféricas suelen emplearse para estudiar sistemas reales anisotrópicos. Su importancia práctica puede observarse en situaciones variadas como la extracción de fluidos supercríticos [1,2], en el equilibrio de fases de nematógenos [3,4], e incluso en el análisis de interacciones del tipo polar [5-7].

Para gases simples, o ligeramente densos, dos potenciales intermoleculares empleados regularmente son el potencial por pares de multicentros $[8,9]$ y el potencial de Kihara [10]. En el primer caso la molécula es representada por un conjunto de sitios enlazados que interactúan con los sitios de otra molécula; la energía de interacción de este modelo tiene una dependencia con la distancia entre sitios y con su orientación relativa. En el segundo caso la forma de la molécula se establece mediante un cuerpo convexo por lo que la termodinámica de dichos sistemas es escrita en términos de las características geométricas del cuerpo usado. Isihara [11] introdujo por vez primera este concepto y es desarrollado posteriormente por él mismo et. al. [12,13] y por Kihara [14-17] para la obtención analítica de los primeros coeficientes viriales de fluidos formados por cuerpos convexos. La dependencia única con la distancia permite escribir de forma analítica el segundo coeficiente virial y para el caso de interacciones no aditivas puede hacerse lo mismo para el tercer coeficiente [18,19]; sin embargo, el conocimiento de coeficientes viriales de mayor orden está limitado a resultados numéricos para modelos particulares.

La dificultad matemática en el cálculo exacto de los primeros coeficientes viriales lleva subsecuentemente al uso de teoría de perturbaciones y a la simulación de sistemas específicos para obtener expresiones numéricas de coeficientes viriales de mayor orden y lograr incluso la construcción de la ecuación de estado para fluidos formados por cuerpos convexos de interés; investigaciones en esta dirección han sido desarrolladas por Boublik et. al. [20-23], Nezbeda [24,25] y Vega et. al. [26-29]. Trabajos recientes de Wertheim [30], han impulsado el estudio de estos sistemas desde un punto de vista más riguroso cuyo formalismo matemático permite calcular teóricamente el tercer coeficiente virial, aunque en el cálculo numérico de coeficientes de mayor orden se presentan inconvenientes computacionales [31].

Desde el punto de vista del principio de estados correspondientes, los modelos esféricamente simétricos han mostrado una buena eficacia en la fase gaseosa. Muestra de lo anterior se ha hecho patente al estudiar moléculas simples $\left(\mathrm{H}_{2}, \mathrm{~N}_{2}, \mathrm{CO}_{2}\right.$ y $\left.\mathrm{C}_{2} \mathrm{H}_{4}\right)$ las cuales no son totalmente esféricas, empleando modelos sencillos como el potencial LJ [14,32-34]. Sin embargo, dichos modelos esféricos fallan a medida que las moléculas del sistema se alejan de la esfericidad y/o la densidad del fluido aumenta, lo que origina desviaciones con respecto a dicho principio. Uno de los primeros intentos en este sentido fue llevado a cabo por Cook et. al. [35] trabajando con un potencial efectivo obtenido a partir de promediar angularmente una función realista y dependiente de la orientación de las partículas, y aplicándolo a moléculas con simetría axial.

En este capítulo se presenta el trabajo con sistemas formados por cuerpos convexos en fase gaseosa. Los 
cuerpos interactúan mediante potenciales dependientes principalmente de su elongación y de la distancia mínima que separa sus superficies. En primera instancia se hace una extensión a lo obtenido por del Río et. al. [36], con su reinterpretación del concepto ANC en términos de cuerpos convexos, para hallar nuevos aspectos teóricos y prácticos acerca de la teoría de potenciales efectivos. Se calcula el segundo coeficiente virial para interacciones de pozo cuadrado convexo y se muestra el comportamiento con respecto a la elongación del cuerpo y su dependencia con las características del nódulo central. Como más adelante se mostrará, se puede establecer una equivalencia entre este modelo y el de Kihara la cual logra vincular los conceptos de la teoría de Kihara con los volúmenes reducidos de colisión presentes en la teoría efectiva ANC. Posteriormente, empleando una interacción continua, también se halla una conexión directa entre la teoría elaborada por Kihara y los parámetros ANC. Dicho vínculo permite encontrar una relación cuantitativa entre el número de carbonos que forma una cadena molecular con la elongación del modelo ANC [37], y aplicarla a casos reales.

Parte del presente trabajo consistirá, precisamente, en retomar esta visión de modelar moléculas mediante cuerpos convexos. Primero se tomará la interacción pozo cuadrado convexo y se comparará con la versión implementada por Kihara para sistemas similares mostrando que ambos modelos tienen la misma base conceptual. Posteriormente, se trabajará con potenciales continuos del tipo ANC donde el nódulo central puede ser cualquier cuerpo convexo. Nuevamente se hallarán equivalencias entre esta manera de trabajar con la termodinámica de cuerpos convexos desarrollada por Kihara; interés especial se tendrá para cuerpos esferocilíndricos y elipsoidales, tanto prolatos como oblatos, por su aplicación directa a sistemas gaseosos de n-alcanos y n-perfluoroalcanos.

\subsection{Antecedentes}

El potencial Kihara fue introducido como una generalización al LJ, ec. 2.12, para el estudio de moléculas anisotrópicas y ha sido empleado continuamente para investigar la termodinámica, la estructura y las propiedades de transporte de los fluidos de moléculas representadas por cuerpos convexos. El potencial de interacción Kihara incorpora un pozo atractivo suave alrededor del nódulo duro dado por la ecuación 2.15 y con dependencia explícita con la distancia más corta entre las superficies de los nódulos $\rho$. Los parámetros del potencial han sido obtenidos para distintas sustancias puras y sus mezclas, utilizando una gran variedad de cuerpos convexos como nódulo central; dichos parámetros logran reproducir el segundo coeficiente virial experimental y en algunos casos el tercer coeficiente [10].

Interacciones entre cuerpos convexos han sido consideradas desde el punto de vista ANC [36, 37] mediante los siguientes dos modelos: pozo cuadrado convexo y potencial ANC entre cuerpos convexos. Ambos modelos permiten escribir los volúmenes reducidos $b^{*}$ y $\Lambda^{*}$, sección 3.2.2, en términos de las medidas fundamentales del cuerpo utilizado. Para ambos casos se ha demostrado que el segundo coeficiente virial tiene una dependencia explícita con la elongación de la molécula. Del mismo estudio se encuentra que a medida que la elongación de la molécula aumenta las interacciones de tipo atractivo pierden intensidad haciendo que el potencial efectivo sea mucho más "duro" que el de referencia, es decir, del esférico. Dicha investigación sólo fue aplicada a un sistema formado por esferocilindros prolato quedando pendiente la extensión a cualquier otro cuerpo convexo.

\subsection{Pozos cuadrados convexos y la teoría ANC}

Un cuerpo convexo (CB) está completamente determinado por sus tres medidas fundamentales [10]: $V$, $A$, y $M$; volumen, área superficial y radio de curvatura medio, respectivamente. En general, dichas medidas fundamentales son escritas en función del ancho del cuerpo $h$ y de su elongación $\xi=L / h$, siendo $L$ el largo ${ }^{1}$

\footnotetext{
${ }^{1}$ Un cuerpo convexo se caracteriza como aquél en el que cualquier segmento rectilíneo que une dos de sus puntos queda completamente contenido en él [16,38].
} 
El modelado de moléculas no esféricas empleando cuerpos convexos requiere de algunos elementos teóricos presentados en la sección C.2 del apéndice. Al emplear esta información, las propiedades termodinámicas de un gas formado por tales cuerpos estarán escritas en términos de las características geométricas de los mismos. En esta sección se emplea dicho enfoque en gases cuyas moléculas interactúan mediante una generalización angular del pozo cuadrado.

\subsubsection{Interacción CSW}

Sean $h_{1}$ y $h_{2}$ dos cuerpos convexos del mismo ancho $h$, y con la misma elongación $\xi_{h}$, que interactúan mediante el siguiente potencia $\left.\right|^{2}$

$$
\Phi_{\mathrm{CSW}}(r, \Omega)=\left\{\begin{array}{cc}
\infty, & r<l_{h}\left(\Omega ; h, \xi_{h}\right), \\
-\epsilon, & l_{h}\left(\Omega ; h, \xi_{h}\right)<r<l_{H}\left(\Omega ; H, \xi_{H}\right), \\
0, & l_{H}\left(\Omega ; H, \xi_{H}\right)<r,
\end{array}\right.
$$

denominado potencial de pozo cuadrado convexo (CSW). Este potencial es una generalización angular del potencial SW dado en la sección 2.2.3 El símbolo $\Omega$ representa la orientación relativa entre las dos partículas y $r$ es la distancia entre sus centros de masa.

El cuerpo convexo $H$ formado por todos los puntos cuyas distancias desde la superficie de $h_{1}$ sean iguales o menores que una cantidad $\rho_{0}$, se llama CB paralelo a $h_{1}$ (véase figura C.1 y notación de la sección C.2. Lo anterior significa que el ancho del cuerpo $H$ es $h+2 \rho_{0}$, del cual

$$
\rho_{0}=\frac{H-h}{2}=\frac{h}{2}\left(\lambda_{0}-1\right)
$$

donde $\lambda_{0}=H / h$ define el ancho relativo del pozo del potencial y por lo mismo, la diferencia $l_{H}(\Omega)-l_{h}(\Omega)=2 \rho_{0}$ es constante.

Si se fija el cuerpo $H$ y se desliza a $h_{2}$ alrededor del primero manteniendo una orientación constante, el centro de gravedad del segundo forma la superficie de un nuevo cuerpo convexo que se denotará $h+\rho_{0}+h$. Finalmente, promediando angularmente sobre todas las configuraciones posibles para $h_{2}$, los correspondientes volúmenes de colisión ANC se obtienen sustituyendo el potencial CSW en las ecuaciones [3.1) y [3.2 [36]. Los promedios angulares resultantes son obtenidos analíticamente usando la ecuación C.10; es decir,

$$
2 \sigma^{3}=2\left\langle l_{h}^{3}(\Omega)\right\rangle=\left\langle V_{h+h}\right\rangle
$$

y

$$
2 R^{3}=2\left\langle l_{H}^{3}(\Omega)\right\rangle=\left\langle V_{h+\rho_{0}+h}\right\rangle ;
$$

los cuales pueden ser escritos en términos de los parámetros $h$ y $\xi_{h}$ del cuerpo duro central.

Para modelar moléculas, el nódulo de CSW representará la repulsión o volumen excluido que surgen cuando las superficies de dos moléculas se acercan la una a la otra; por su parte el cascarón del CSW atractivo es usado para modelar la atracción tipo van der Waals entre las mismas moléculas. Con esta imagen el ancho del pozo atractivo de van der Waals es uniforme alrededor de la molécula.

\subsubsection{Segundo coeficiente virial}

Combinando los volúmenes anteriores $\sigma^{3}$ y $R^{3}$ de la interacción CSW, junto con las ecuaciones 3.11 3.13 de la sección 3.2 , se obtiene directamente el segundo coeficiente virial

$$
B_{\mathrm{CSW}}\left(T^{*}, \xi_{h}\right)=\left(V_{h}+\frac{M_{h} A_{h}}{4 \pi}\right) \mathrm{e}^{1 / T^{*}}-\left\{V_{h}+\frac{M_{h} A_{h}}{4 \pi}+\left(A_{h}+\frac{M_{h}^{2}}{4 \pi}\right) \rho_{0}+M_{h} \rho_{0}^{2}+\frac{2 \pi}{3} \rho_{0}^{3}\right\}\left(\mathrm{e}^{1 / T^{*}}-1\right),
$$

\footnotetext{
${ }^{2}$ Esta es la definición presentada por del Río et. al. [36].
} 
donde $T^{*}=k_{\mathrm{B}} T / \epsilon, \rho_{0}$ está dado por la ecuación (5.2), y $V_{h}, A_{h}$ y $M_{h}$ son las medidas fundamentales del nódulo central, las cuales introducen la dependencia con su elongación $\xi_{h}{ }^{3}$

Usando a $\frac{1}{2}\left\langle V_{h+h}\right\rangle$ como volumen de reducción, el segundo coeficiente virial, ec. [5.5), puede ser escrito como

$$
B_{\mathrm{CSW}}^{*}\left(T^{*}, \xi_{h}\right)=\frac{B_{\mathrm{CSW}}\left(T^{*}\right)}{\frac{1}{2}\left\langle V_{h+h}\right\rangle}=1-V^{*}\left(\xi_{h}, \lambda_{0}\right)\left(\mathrm{e}^{1 / T^{*}}-1\right)
$$

donde

$$
V^{*}\left(\xi_{h}, \lambda_{0}\right)=\frac{\left\langle V_{h+\rho_{0}+h}\right\rangle}{\left\langle V_{h+h}\right\rangle}-1 .
$$

La ecuación (5.6) dará el segundo coeficiente virial reducido para cualquier CB cuyas cantidades fundamentales sean conocidas. Dicho coeficiente tendrá así una dependencia con la temperatura, la elongación del nódulo central y el ancho $\rho_{0}$ del pozo (o $\lambda_{0}$, vía la ecuación 5.2 .

\subsubsection{Temperatura de Boyle}

La temperatura de Boyle se define como aquella a la cual el segundo coeficiente virial es cero: $B\left(T_{\mathrm{B}}^{*}\right)=0$; es decir, aquella temperatura en la cual el segundo coeficiente cambia de signo. Empleando esta condición, y partiendo de la ec. (5.6), el modelo anterior permite calcular dicha temperatura en función de la elongación del nódulo duro tal que

$$
\frac{1}{T_{\mathrm{B}}^{*}\left(\xi_{h}\right)}=\ln \left[\frac{V^{*}\left(\xi_{h}, \lambda_{0}\right)+1}{V^{*}\left(\xi_{h}, \lambda_{0}\right)}\right]
$$

donde $V^{*}\left(\xi_{h}, \lambda_{0}\right)$ se ha definido en (5.7).

\subsubsection{Función suavidad}

Para una elongación dada, la curva paramétrica entre el segundo coeficiente virial (5.6) con respecto a uno de referencia $\left(\xi_{h}=1\right)$ presenta el comportamiento lineal hallado comúnmente en la teoría ANC, figura 5.1 .

Para hallar la relación matemática entre ambos coeficientes, se propone la expresión

$$
B_{\mathrm{CSW}}^{*}\left(T^{*}, \xi_{h}\right)=R+S B_{\mathrm{CSW}}^{*}\left(T^{*}, 1\right)
$$

donde $R$ y $S$ son los parámetros característicos de una recta en el plano. Si se usa el hecho de que $B_{\mathrm{CSW}}^{*}\left(T_{\mathrm{B}}^{*}, 1\right)=0$ y, usando la temperatura de Boyle para el sistema de referencia, es fácil verificar que

$$
R=1-\frac{V^{*}\left(\xi_{h}, \lambda_{0}\right)}{V^{*}\left(1, \lambda_{0}\right)}
$$

a partir de la cual se obtiene

$$
S=\frac{V^{*}\left(\xi_{h}, \lambda_{0}\right)}{V^{*}\left(1, \lambda_{0}\right)}
$$

por lo que la ecuación 5.9 se escribe en la forma final

$$
B_{\mathrm{CSW}}^{*}\left(T^{*}, \xi_{h}\right)=1-S+S B_{\mathrm{CSW}}^{*}\left(T^{*}, 1\right)
$$

que es similar a la dada por la teoría ANC, ec. (3.26), correspondiendo el parámetro $S$ de la ecuación (5.11) con el de la suavidad. En este caso, $B_{0}^{*}\left(T^{*}\right)=B_{\mathrm{CSW}}^{*}\left(T^{*}, 1\right)$, corresponde al segundo coeficiente virial de una esfera.

\footnotetext{
${ }^{3}$ La misma expresión se halla usando un caso especial del potencial de pozo cuadrado usado por Kihara [10,18], y en el apéndice A.2.2 se muestra el desarrollo.
} 


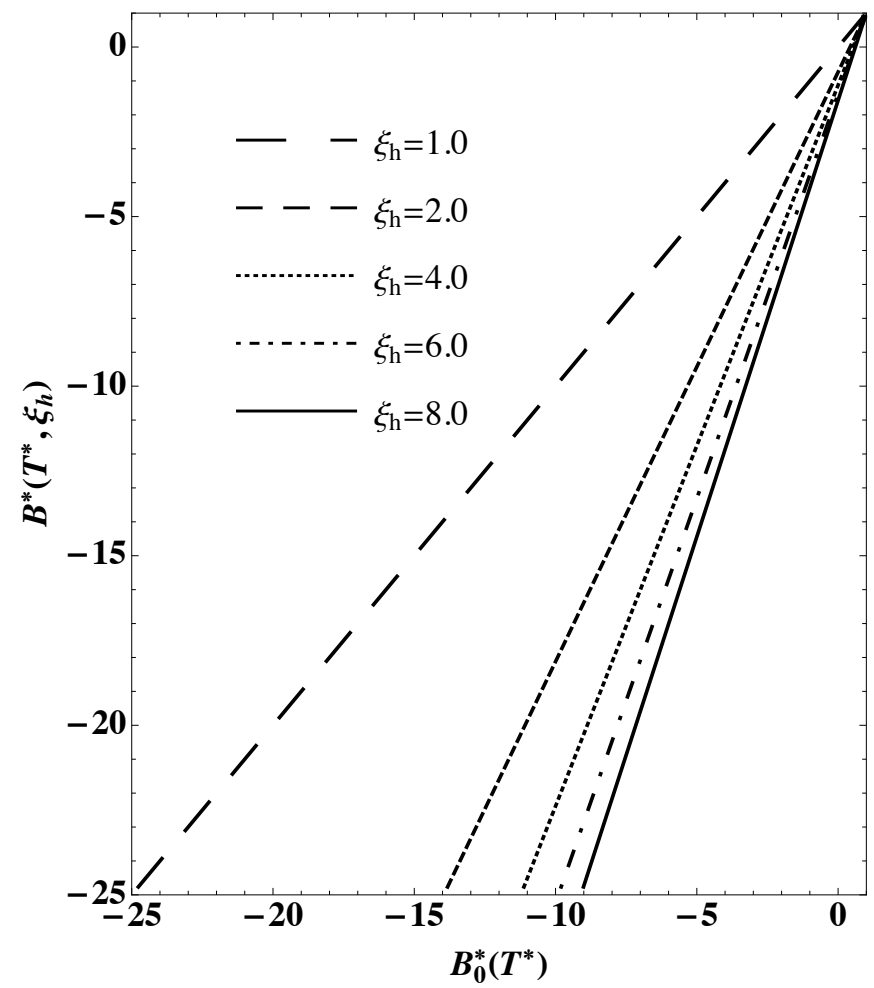

Figura 5.1: Relación lineal entre $B_{\mathrm{CSW}}^{*}\left(T^{*}, \xi_{h}\right)$ y el de referencia $B_{\mathrm{CSW}}^{*}\left(T^{*}, \xi_{h}=1.0\right)$. Las distintas curvas pertenecen a distintas elongaciones y se han construido usando temperaturas reducidas en el intervalo $[0.3$, 10.0].

\subsection{Potenciales ANC para moléculas convexas}

El proceso anterior puede ser generalizado a potenciales continuos considerando partículas que interactúan de forma más realista que el caso CSW. La construcción de los volúmenes reducidos $b^{*}\left(T^{*}\right)$ y $\Lambda^{*}\left(T^{*}\right)$ para esta nueva función potencial permite relacionarla con la teoría ANC y por lo mismo se le asignará el nombre de interacción de cuerpos convexos ANC (CANC). Se plantea el proceso completo para cualquier cuerpo convexo, y con dicha interacción se calculan el segundo coeficiente virial y la función suavidad tanto para la zona repulsiva como para la atractiva. Posteriormente se construye una función suavidad que relaciona los segundos coeficientes viriales de forma lineal, enfocándose el trabajo final en aquellos cuerpos que representen efectivamente a una molécula lineal. La elongación, como se verá, se convierte nuevamente en el parámetro principal en términos del cual se escribe la termodinámica de aquel gas compuesto de tales moléculas.

\subsubsection{Interacción CANC}

El caso CSW revisado en la sección anterior se generaliza construyendo una interacción discontinua entre dos CB de ancho $h$ y elongación $\xi_{h}$, ambos con medidas fundamentales: $V_{h}, A_{h}, M_{h}$. Este potencial discreto tiene la forma

$$
\Phi(r, \Omega)=\left\{\begin{array}{cc}
\infty & r<r_{1}(\Omega) \\
\epsilon_{n} & r_{n}(\Omega)<r<r_{n+1}(\Omega) \\
0 & r>r_{c}(\Omega)
\end{array}\right.
$$


donde $r$ es la distancia entre sus centros de masa, $\Omega$ la orientación relativa entre ellos y $\epsilon_{n}$ es la profundidad del respectivo " $n$-escalón". Se considerará una zona repulsiva por debajo de un escalón $m$-ésimo y por arriba de éste sólo intervienen fuerzas atractivas de manera tal que en el c-ésimo escalón la interacción se anula.

Para una $\Omega$ fija, la derivada parcial del factor de Boltzmann con respecto a la distancia $r$ puede escribirse como [36]

$$
\frac{\partial \mathrm{e}^{-\Phi(r, \Omega) / k_{\mathrm{B}} T}}{\partial r}=\sum_{n=0}^{c} \delta\left(r-r_{n}(\Omega)\right)\left[\mathrm{e}^{-\epsilon_{n} / k_{\mathrm{B}} T}-\mathrm{e}^{-\epsilon_{n-1} / k_{\mathrm{B}} T}\right]
$$

la cual sustituida en las integrales de ANC (3.9) y 3.10) (previa integración por partes) permite escribirlas como

$$
\sigma^{3} \mathrm{e}^{\epsilon / k_{\mathrm{B}} T}=\sum_{n=0}^{m}\left\langle r_{n}^{3}(\Omega)\right\rangle\left[\mathrm{e}^{-\epsilon_{n} / k_{\mathrm{B}} T}-\mathrm{e}^{-\epsilon_{n-1} / k_{\mathrm{B}} T}\right]
$$

y

$$
R^{3}\left(\mathrm{e}^{\epsilon / k_{\mathrm{B}} T}-1\right)=-\sum_{n=m+1}^{\mathrm{c}}\left\langle r_{n}^{3}(\Omega)\right\rangle\left[\mathrm{e}^{-\epsilon_{n} / k_{\mathrm{B}} T}-\mathrm{e}^{-\epsilon_{n-1} / k_{\mathrm{B}} T}\right]
$$

El parámetro $\left\langle r_{n}^{3}(\Omega)\right\rangle$ se obtiene de forma similar al caso CSW. Así, uno de los cuerpos $h$ se mantiene fijo mientras un segundo se traslada alrededor del primero manteniendo constantes tanto la orientación como la distancia entre superficies $\Delta \rho_{n}=n\left(r_{n}-r_{n-1}\right)$. Así, el $n$-ésimo escalón del potencial caracteriza espacialmente al $n$-cuerpo convexo paralelo a $h$; esto es, $\left\langle r_{n}^{3}(\Omega)\right\rangle=\left\langle V_{h+\Delta \rho_{n}+h}\right\rangle$. Esto significa que al realizar los promedios angulares anteriores habrá una dependencia única con la distancia $\rho$ entre sus superficies. Posteriormente, al tomar los límites: $n \rightarrow \infty, \Delta \rho_{n} \rightarrow 0$, y $\Delta \epsilon \rightarrow 0$, las sumas anteriores pueden escribirse como

$$
2 \sigma^{3} \mathrm{e}^{\epsilon / k_{\mathrm{B}} T}=\int_{0}^{\rho_{0}} d \rho\left\langle V_{h+\rho+h}\right\rangle \frac{\partial \mathrm{e}^{-\Phi(\rho) / k_{\mathrm{B}} T}}{\partial \rho}
$$

y

$$
2 R^{3}\left(\mathrm{e}^{\epsilon / k_{\mathrm{B}} T}-1\right)=-\int_{\rho_{0}}^{\infty} d \rho\left\langle V_{h+\rho+h}\right\rangle \frac{\partial \mathrm{e}^{-\Phi(\rho) / k_{\mathrm{B}} T}}{\partial \rho}
$$

donde el potencial $\Phi(\rho)$ es un potencial esférico continuo dependiente de la mínima distancia $\rho$ entre las superficies de los cuerpos convexos. $\rho_{0}$ identifica la posición del mínimo, tal que $\Phi\left(\rho_{0}\right)=-\epsilon$ y se ha hecho coincidir con $r_{m}=r_{m+1}$ del potencial discreto, quedando todo en términos del nódulo central $h$.

Mediante una integración por partes las dos ecuaciones de arriba pueden expresarse como

$$
2 \sigma^{3} \mathrm{e}^{\epsilon / k_{\mathrm{B}} T}=\left\langle V_{h+\rho_{0}+h}\right\rangle\left[\mathrm{e}^{\epsilon / k_{\mathrm{B}} T}-1\right]+\left\langle V_{h+h}\right\rangle-\int_{0}^{\rho_{0}} d \rho\left\langle A_{h+\rho+h}\right\rangle\left[\mathrm{e}^{-\Phi(\rho) / k_{\mathrm{B}} T}-1\right]
$$

y

$$
2 R^{3}\left[\mathrm{e}^{\epsilon / k_{\mathrm{B}} T}-1\right]=\left\langle V_{h+\rho_{0}+h}\right\rangle\left[\mathrm{e}^{\epsilon / k_{\mathrm{B}} T}-1\right]+\int_{\rho_{0}}^{\infty} d \rho\left\langle A_{h+\rho+h}\right\rangle\left[\mathrm{e}^{-\Phi(\rho) / k_{\mathrm{B}} T}-1\right]
$$

cuya combinación ANC de acuerdo a (3.3) permite recuperar al segundo coeficiente virial obtenido por la teoría convencional de cuerpos convexos, ec. A.28, pág. 109.

La forma exacta de $\left\langle V_{h+\rho+h}\right\rangle$ y $\left\langle A_{h+\rho+h}\right\rangle$ aparecen en las ecuaciones C.10 y C.11 del apéndice C.2 Si el potencial $\Phi(\rho)$ es del tipo LJ 2.12) 4 las integrales involucradas tienen solución analítica dependiente de la temperatura. Introduciendo las cantidades reducidas $T^{*}=k_{\mathrm{B}} T / \epsilon$ y $\Phi^{*}=\Phi / \epsilon$ es sencillo demostrar que los diámetros de colisión pueden escribirse como

$$
\begin{aligned}
\sigma^{3}= & \frac{1}{2}\left\langle V_{h+h}\right\rangle \mathrm{e}^{-\frac{1}{T^{*}}}+\frac{1}{2}\left\langle V_{h+\rho_{0}+h}\right\rangle\left(1-\mathrm{e}^{-\frac{1}{T^{*}}}\right) \\
& -\mathrm{e}^{-\frac{1}{T^{*}}}\left\{\left(A_{h}+\frac{M_{h}^{2}}{4 \pi}\right) \rho_{0}\left(\mathcal{D}_{1}\left(T^{*}\right)-1\right)+M_{h} \rho_{0}^{2}\left(\mathcal{D}_{2}\left(T^{*}\right)-1\right)+\frac{2 \pi \rho_{0}^{3}}{3}\left(\mathcal{D}_{3}\left(T^{*}\right)-1\right)\right\}
\end{aligned}
$$

\footnotetext{
${ }^{4}$ Es decir, el potencial de Kihara.
} 
y

$$
R^{3}=\frac{1}{2}\left\langle V_{h+\rho_{0}+h}\right\rangle+\left(\mathrm{e}^{\frac{1}{T^{*}}}-1\right)^{-1}\left\{\left(A_{h}+\frac{M_{h}^{2}}{4 \pi}\right) \rho_{0} \mathcal{E}_{1}\left(T^{*}\right)+M_{h} \rho_{0}^{2} \mathcal{E}_{2}\left(T^{*}\right)+\frac{2 \pi}{3} \rho_{0}^{3} \mathcal{E}_{3}\left(T^{*}\right)\right\}
$$

donde las integrales $\mathcal{D}_{k}\left(T^{*}\right)$ y $\mathcal{E}_{k}\left(T^{*}\right)$ son las mismas que aparecen en la sección de la teoría ANC, y su representación semianalítica para ambas integrales ha sido planteada y resuelta como se muestra en el apéndice B.2.2 pág. 112 Como puede observarse, estos volúmenes tienen dependencia con la temperatura, las propiedades geométricas del nódulo central $h$, así como del mínimo, $\rho_{0}$ y $\epsilon$, del potencial continuo.

\subsubsection{Volúmenes de colisión}

Considérense dos cuerpos convexos paralelos entre sí, separados una distancia $\rho_{0}$ de sus superficies. El cuerpo central (ancho $h$ ) modelará la molécula y el cuerpo paralelo externo (ancho $H$ ) se ubica precisamente a la distancia donde se halla el mínimo energético del potencial $\Phi(\rho)$; es decir, $H=h+2 \rho_{0}$. Al primer cuerpo se le denominará cuerpo $h$ y al segundo cuerpo $H$. Si se introduce la razón $\lambda_{0}=\frac{H}{h}>1$ y por simplicidad se emplea el volumen $\frac{2 \pi}{3} h^{3}$ como unidad de reducción, entonces los volúmenes 5.21) y 5.22 se escriben de forma adimensional

$$
\begin{aligned}
b^{*}= & \frac{\sigma^{3}}{\frac{2 \pi}{3} h^{3}}= \\
= & \frac{\left\langle V_{h+h}\right\rangle}{\frac{4 \pi}{3} h^{3}} \mathrm{e}^{-\frac{1}{T^{*}}}+\frac{\left\langle V_{h+\rho_{0}+h}\right\rangle}{\frac{4 \pi}{3} h^{3}}\left(1-\mathrm{e}^{-\frac{1}{T^{*}}}\right)-\mathrm{e}^{-\frac{1}{T^{*}}}\left\{\left(\frac{A_{h}+\frac{M_{h}^{2}}{4 \pi}}{\frac{2 \pi}{3} h^{2}}\right)\left(\frac{\lambda_{0}-1}{2}\right)\left(\mathcal{D}_{1}\left(T^{*}\right)-1\right)\right. \\
& \left.+\frac{M_{h}}{\frac{2 \pi}{3} h}\left(\frac{\lambda_{0}-1}{2}\right)^{2}\left(\mathcal{D}_{2}\left(T^{*}\right)-1\right)+\left(\frac{\lambda_{0}-1}{2}\right)^{3}\left(\mathcal{D}_{3}\left(T^{*}\right)-1\right)\right\}
\end{aligned}
$$

y

$$
\begin{aligned}
\Lambda^{*}= & \frac{R^{3}}{\frac{2 \pi}{3} h^{3}}= \\
= & \frac{\left\langle V_{h+\rho_{0}+h}\right\rangle}{\frac{4 \pi}{3} h^{3}}+\frac{1}{\mathrm{e}^{\frac{1}{T^{*}}}-1}\left\{\left(\frac{A_{h}+\frac{M_{h}^{2}}{4 \pi}}{\frac{2 \pi}{3} h^{2}}\right)\left(\frac{\lambda_{0}-1}{2}\right) \mathcal{E}_{1}\left(T^{*}\right)+\frac{M_{h}}{\frac{2 \pi}{3} h}\left(\frac{\lambda_{0}-1}{2}\right)^{2} \mathcal{E}_{2}\left(T^{*}\right)\right. \\
& \left.+\left(\frac{\lambda_{0}-1}{2}\right)^{3} \mathcal{E}_{3}\left(T^{*}\right)\right\}
\end{aligned}
$$

dejándolos en función de la elongación $\xi_{h}$ del nódulo central y del parámetro $\lambda_{0}$.

\subsubsection{Segundo coeficiente virial}

El método anterior puede emplearse para cualquier cuerpo convexo cuyas medidas fundamentales sean conocidas. El segundo coeficiente virial de este tipo de interacciones se obtiene combinando las ecuaciones 5.23 y 5.24 con la ecuación 3.13 pág. 33). Como posteriormente se demuestra, el comportamiento del segundo coeficiente virial CANC (para cualquier elongación y para cualquier CB aquí empleado) con respecto al coeficiente virial de la referencia es aproximadamente lineal tal como lo establece la teoría ANC. A modo de ejemplo, en la figura 5.2 se presenta un conjunto de datos $B^{*}\left(T^{*}, \xi_{h}\right)$ vs $B_{0}^{*}\left(T^{*}\right)$, para distintas temperaturas y elongaciones, de un sistema de elipsoides prolato. Como se observa en la figura para elongaciones grandes y/o a temperaturas bajas (conjunto de puntos extremos superiores) la linealidad entre ambos coeficientes ya no es exacta; sin embargo, se considerará la relación lineal como una aproximación. 


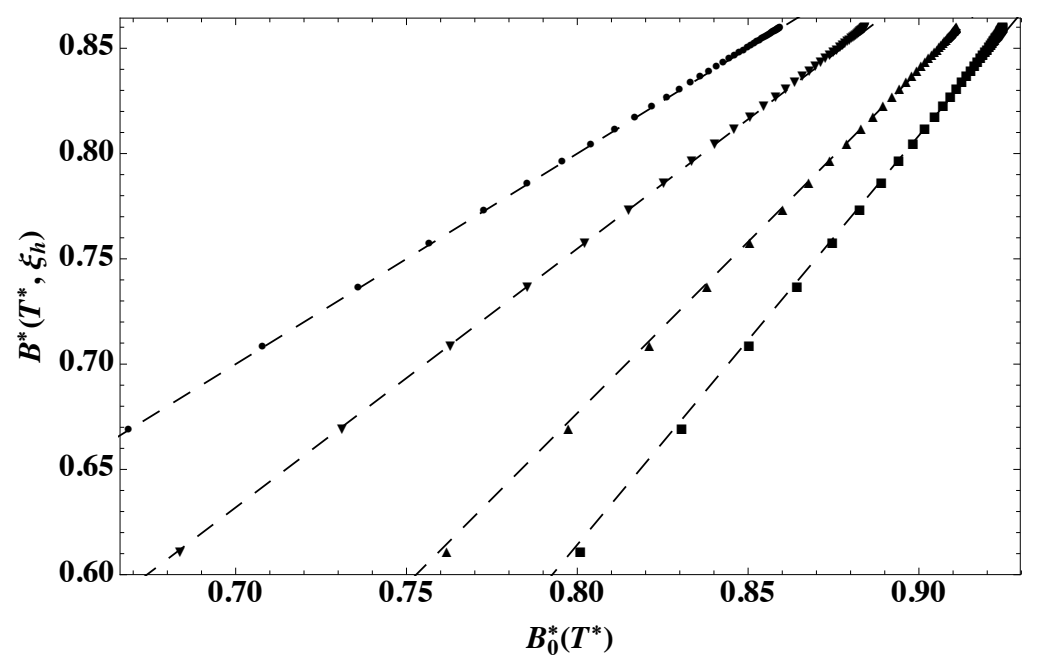

Figura 5.2: Comportamiento lineal entre los coeficientes viriales con distintas elongaciones y la referencia esférica para un elipsoide prolato: $\xi_{h}=1.0(\bullet), 2.0(\boldsymbol{\nabla}), 5.0(\boldsymbol{\Lambda})$ y $10.0(\boldsymbol{\nabla})$. Los puntos superiores pertenecen a temperaturas bajas. Las líneas rectas a trazos son para ayudar a la vista.

\subsubsection{Suavidad repulsiva y atractiva}

Para hallar de manera explícita expresiones para las suavidades $\left(S_{\mathrm{R}}\right.$ y $\left.S_{\mathrm{A}}\right)$ en función de la elongación de la partícula se usan las relaciones lineales aproximadas dadas por

$$
\begin{aligned}
& \mathrm{e}^{-1 / T^{*}} \mathcal{D}_{1}\left(T^{*}\right)=\alpha_{1}+\alpha_{2} \mathrm{e}^{-1 / T^{*}} \mathcal{D}_{3}\left(T^{*}\right) \\
& \mathrm{e}^{-1 / T^{*}} \mathcal{D}_{2}\left(T^{*}\right)=\beta_{1}+\beta_{2} \mathrm{e}^{-1 / T^{*}} \mathcal{D}_{3}\left(T^{*}\right)
\end{aligned}
$$

para la parte repulsiva, $\mathrm{y}$

$$
\begin{aligned}
& {\left[\mathrm{e}^{1 / T^{*}}-1\right]^{-1} \mathcal{E}_{1}\left(T^{*}\right)=\alpha_{3}+\alpha_{4}\left[\mathrm{e}^{1 / T^{*}}-1\right]^{-1} \mathcal{E}_{3}\left(T^{*}\right)} \\
& {\left[\mathrm{e}^{1 / T^{*}}-1\right]^{-1} \mathcal{E}_{2}\left(T^{*}\right)=\beta_{3}+\beta_{4}\left[\mathrm{e}^{1 / T^{*}}-1\right]^{-1} \mathcal{E}_{3}\left(T^{*}\right)}
\end{aligned}
$$

para la atractiva, ver figura 5.3 . donde $\alpha_{i}$ y $\beta_{i}$ son parámetros (ver tabla 5.1 que ajustan a una serie de puntos obtenidos por parametrización de ambas cantidades en el intervalo de temperatura: $0.3 \leq T^{*} \leq 10.0$.

\begin{tabular}{|c|c|c|}
\hline$i$ & $\alpha_{i}$ & $\beta_{i}$ \\
\hline 1 & -0.021 & -0.018 \\
\hline 2 & 0.452 & 0.775 \\
\hline 3 & 0.059 & 0.082 \\
\hline 4 & 0.150 & 0.432 \\
\hline
\end{tabular}

Tabla 5.1: Parámetros $\alpha_{i}$ y $\beta_{i}$ para suavidades repulsiva y atractiva.

Sustituyendo estos ajustes en las ecuaciones (5.23) y (5.24), y después de un proceso algebraico sencillo, los volúmenes de colisión reducidos pueden presentarse en una forma muy similar a las ecs. (3.23) y (3.24) 


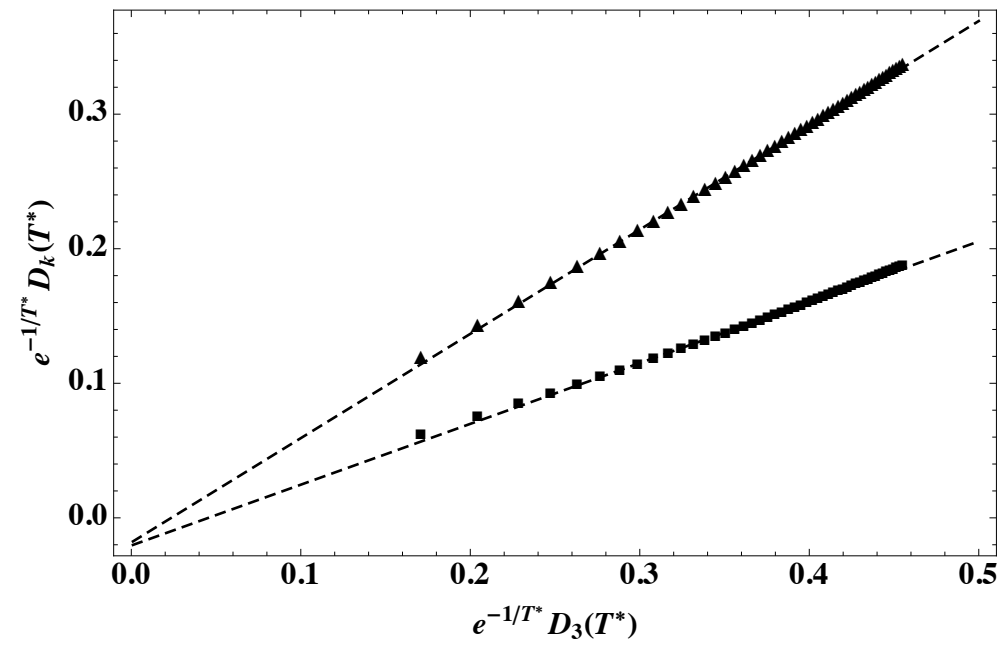

Figura 5.3: Relación lineal entre $\mathrm{e}^{-1 / T^{*}} \mathcal{D}_{k}\left(T^{*}\right)$ vs $\mathrm{e}^{-1 / T^{*}} \mathcal{D}_{3}\left(T^{*}\right): k=1(\mathbf{\square}), k=2(\boldsymbol{\Lambda})$. Un comportamiento similar se halla para el caso atractivo. Los puntos superiores corresponden a temperaturas bajas y las líneas rectas a los ajustes (5.27) y (5.28).

pertenecientes a la teoría ANC:

$$
b_{\mathrm{CB}}^{*}\left(T^{*}, \xi_{h}\right)=\frac{b^{*}}{b_{h}^{*}\left(\xi_{h}\right)}=\left[1-f_{\mathrm{R}}\left(\xi_{h}\right)\right]-S_{\mathrm{R}}\left(\xi_{h}\right)+S_{\mathrm{R}}\left(\xi_{h}\right) b_{0}^{*}\left(T^{*}\right)
$$

y

$$
\Lambda_{\mathrm{CB}}^{*}\left(T^{*}, \xi_{h}\right)=\frac{\Lambda^{*}}{b_{h}^{*}\left(\xi_{h}\right)}=\left[1+f_{\mathrm{A}}\left(\xi_{h}\right)\right]-S_{\mathrm{A}}\left(\xi_{h}\right)+S_{\mathrm{A}}\left(\xi_{h}\right) \Lambda_{0}^{*}\left(T^{*}\right) .
$$

donde

$$
b_{h}^{*}\left(\xi_{h}\right)=\frac{\left\langle V_{h+\rho_{0}+h}\right\rangle}{\frac{4 \pi}{3} h^{3}},
$$

con una dependencia extra en $\lambda_{0}$, y la forma exacta del resto de los términos se presentan en las siguientes líneas.

Suavidades CANC. Las funciones que determinan la suavidad para las zonas repulsiva y atractiva están definidas mediante las siguientes dos ecuaciones

$$
\begin{aligned}
& S_{\mathrm{R}}\left(\xi_{h}\right)=\frac{1}{b_{h}^{*}\left(\xi_{h}\right)}\left[\frac{A_{h}+\frac{M_{h}^{2}}{4 \pi}}{\frac{2 \pi}{3} h^{2}}\left(\frac{\lambda_{0}-1}{2}\right) \alpha_{2}+\frac{M_{h}}{\frac{2 \pi}{3} h}\left(\frac{\lambda_{0}-1}{2}\right)^{2} \beta_{2}+\left(\frac{\lambda_{0}-1}{2}\right)^{3}\right] \\
& S_{\mathrm{A}}\left(\xi_{h}\right)=\frac{1}{b_{h}^{*}\left(\xi_{h}\right)}\left[\frac{A_{h}+\frac{M_{h}^{2}}{4 \pi}}{\frac{2 \pi}{3} h^{2}}\left(\frac{\lambda_{0}-1}{2}\right) \alpha_{4}+\frac{M_{h}}{\frac{2 \pi}{3} h}\left(\frac{\lambda_{0}-1}{2}\right)^{2} \beta_{4}+\left(\frac{\lambda_{0}-1}{2}\right)^{3}\right] .
\end{aligned}
$$

Volúmenes de referencia CANC. Los volúmenes $b_{0}^{*}$ y $\Lambda_{0}^{*}$ se definen como

$$
\begin{aligned}
b_{0}^{*}\left(T^{*}, \xi_{h}\right) & =\frac{1}{b_{h}^{*}\left(\xi_{h}\right)}\left[1-\frac{\mathcal{D}_{3}\left(T^{*}\right)}{\mathrm{e}^{1 / T^{*}}}\right] \\
\Lambda_{0}^{*}\left(T^{*}, \xi_{h}\right) & =\frac{1}{b_{h}^{*}\left(\xi_{h}\right)}\left[1+\frac{\mathcal{E}_{3}\left(T^{*}\right)}{\mathrm{e}^{1 / T^{*}}-1}\right] .
\end{aligned}
$$


que son precisamente los volúmenes ANC de referencia, en su versión modificada, ya presentados en la sección 3.5.4. ecs. 3.49) y 3.50).

Funciones extras. Las funciones

$$
f_{\mathrm{R}}\left(\xi_{h}\right)=\frac{1}{b_{h}^{*}\left(\xi_{h}\right)}\left[\frac{A_{h}+\frac{M_{h}^{2}}{4 \pi}}{\frac{2 \pi}{3} h^{2}}\left(\frac{\lambda_{0}-1}{2}\right) \alpha_{1}+\frac{M_{h}}{\frac{2 \pi}{3} h}\left(\frac{\lambda_{0}-1}{2}\right)^{2} \beta_{1}\right]
$$

y

$$
f_{\mathrm{A}}\left(\xi_{h}\right)=\frac{1}{b_{h}^{*}\left(\xi_{h}\right)}\left[\frac{A_{h}+\frac{M_{h}^{2}}{4 \pi}}{\frac{2 \pi}{3} h^{2}}\left(\frac{\lambda_{0}-1}{2}\right) \alpha_{3}+\frac{M_{h}}{\frac{2 \pi}{3} h}\left(\frac{\lambda_{0}-1}{2}\right)^{2} \beta_{3}\right]
$$

están constituidas por términos que pueden ser despreciados en la mayoría de los casos. Por ejemplo, en el caso del esferocilindro prolato estos dos factores tienen valores máximos de $f_{\mathrm{R}}\left(\xi_{h}\right) \approx-0.02$ y $f_{\mathrm{A}}\left(\xi_{h}\right) \approx 0.03$ para una elongación $\xi_{h}=1.0$ y $\lambda_{0}=3 / 2$.

\subsection{Aplicación a cuerpos convexos específicos}

Todas las propiedades arriba definidas son aplicables a cualquier cuerpo convexo cuyas medidas fundamentales sean conocidas y se expondrán sus comportamientos con respecto a la elongación del nódulo central, $\xi_{h}$. Los cuerpos convexos aquí estudiados, junto con sus medidas fundamentales y algunos de sus parámetros importantes son incluidos en el apéndice C tabla C.1] Dichos cuerpos se identificarán de la siguiente manera: Esferocilindro prolato (PSC), esferocilindro oblato (OSC), elipsoide prolato (PEL), elipsoide oblato (OEL), esferocono (EC) y doble-cono (DC).

En específico, el interés se centrará en aquellos CB que tienden a una esfera cuando $\xi_{h} \rightarrow 1$, la cual será el modelo de referencia; en este grupo caen los esferocilindros y elipsoides. Por su sencillez funcional, el caso prolato para el esferocilindro ocupa gran parte del material aquí presentado, ver tabla C.2. En lo que sigue, los cálculos de las propiedades se harán empleando el valor $\lambda_{0}=3 / 2$, el cual es ampliamente utilizado en el estudio de estos sistemas para gases nobles [10].

\subsubsection{Potencial CSW}

Se usarán aquellos cuerpos cuerpos convexos mostrados en la tabla C.1 En particular, para el ancho de pozo $\lambda_{0}=3 / 2$, las ecuaciones (5.6) y (5.7) dan como resultado las siguientes cantidades

$$
V^{*}\left(1, \frac{3}{2}\right)=\frac{61}{64}, \quad B_{0}^{*}\left(T^{*}\right)=\frac{125-61 \mathrm{e}^{1 / T^{*}}}{64},
$$

donde se ha empleado también la información proveniente de la tabla C.2 En la figura 5.4 se presenta el segundo coeficiente virial CSW de un cuerpo convexo PSC para distintas elongaciones del cuerpo central.

La temperatura de Boyle y la "suavidad" de este sistema pueden ligarse partiendo de las ecuaciones 5.8 y (5.11) respectivas. Ambas ecuaciones estan relacionadas por el volumen $V^{*}\left(\xi_{h}, \lambda_{0}\right)$ por lo que $T_{\mathrm{B}}^{*}$ puede escribirse como

$$
T_{\mathrm{B}}^{*}\left(\xi_{h}\right)=\left[\ln \left(1+\frac{64}{61 S\left(\xi_{h}\right)}\right)\right]^{-1}
$$

en términos del parámetro $S$ del sistema CSW. Esta última relación se encuentra ilustrada en la figura 5.5, en la cual se han graficado (usando la misma escala) las dos propiedades en función de la elongación del nódulo central. 


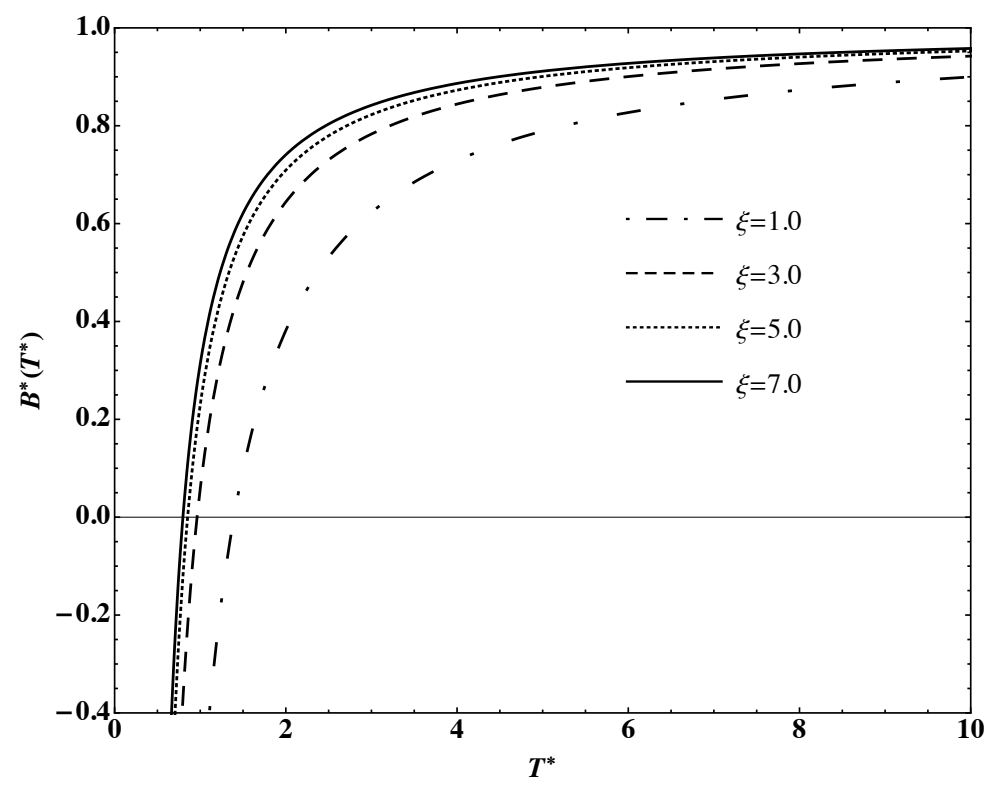

Figura 5.4: Segundo coeficiente virial CSW para un cuerpo convexo PSC con distintas elongaciones. En todos los casos se empleó $\lambda_{0}=3 / 2$.

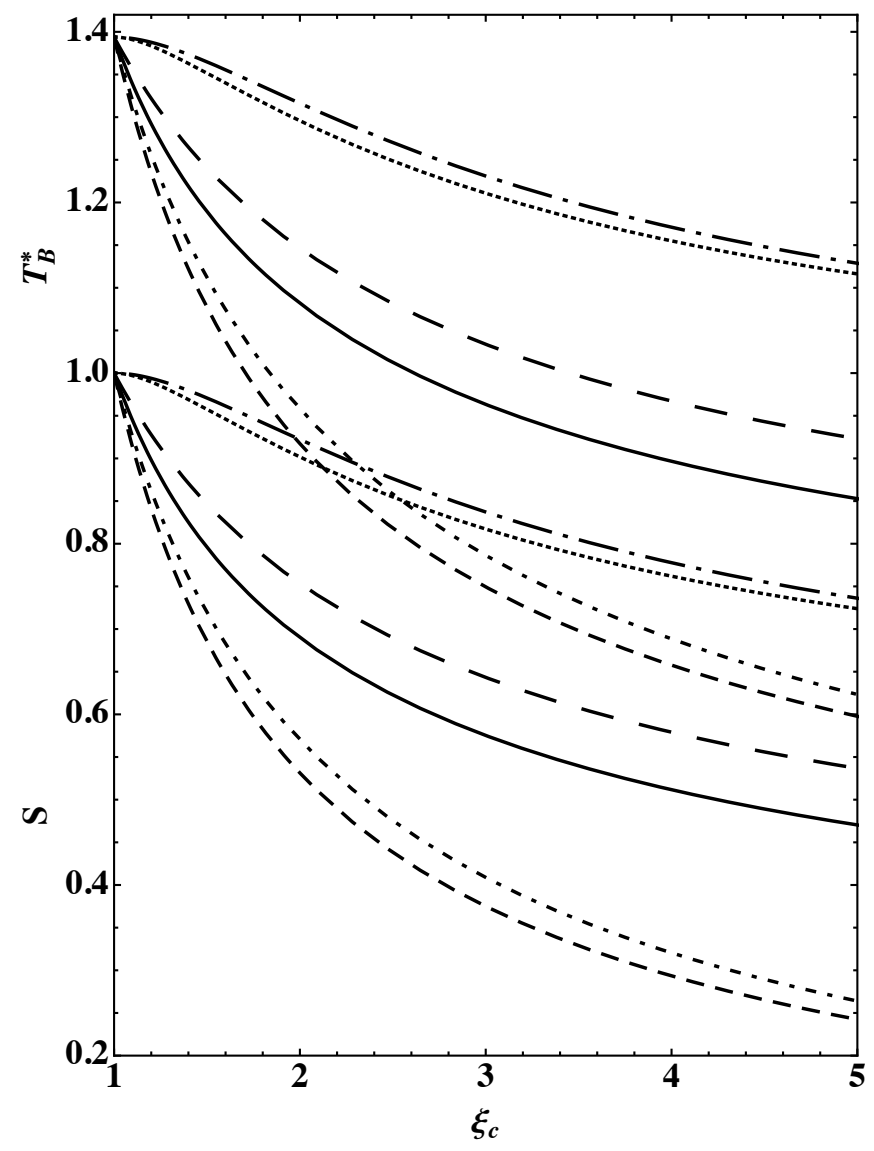

Figura 5.5: Temperatura de Boyle $T_{\mathrm{B}}^{*}\left(\xi_{h}\right)$ (curvas superiores) y $S\left(\xi_{h}\right)$ (curvas inferiores) para un fluido CSW en función de la elongación $\xi_{h}$. Para ambas propiedades se tiene que:

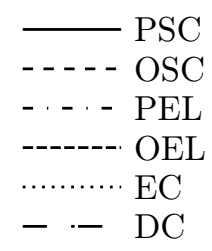

y en todos los casos se ha empleado $\lambda_{0}=3 / 2$. 


\subsubsection{Potencial CANC}

Debido a la sencillez de las medidas fundamentales del cuerpo PSC, la mayoría de los resultados aquí presentados se han enfocado al caso prolato del esferocilindro; sin embargo, en algunos casos se presentan resultados para otros CB también de interés. Al igual que en el caso CSW, se ha empleado $\lambda_{0}=3 / 2$, para mostrar las propiedades características de este sistema.

\section{Volúmenes de colisión}

El comportamiento con respecto a la elongación y a la temperatura para ambos volúmenes reducidos se presenta en las figuras 5.6(a) y 5.6(b) usando esferocilindros y elipsoides prolatos; el comportamiento es similar al obtenido por Ramos et. al. [36] para el primer cuerpo.

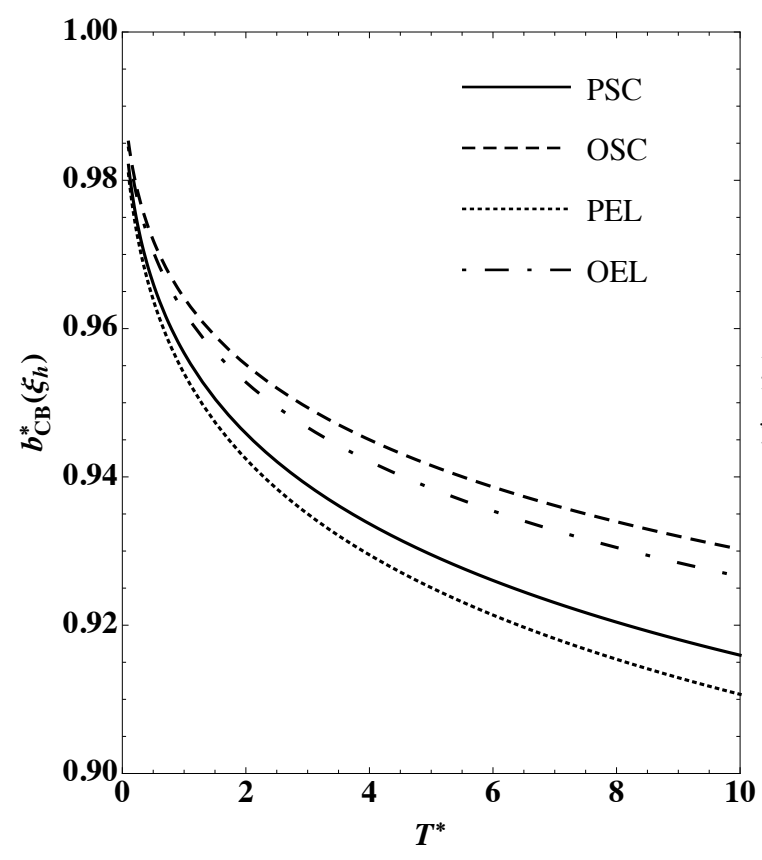

(a)

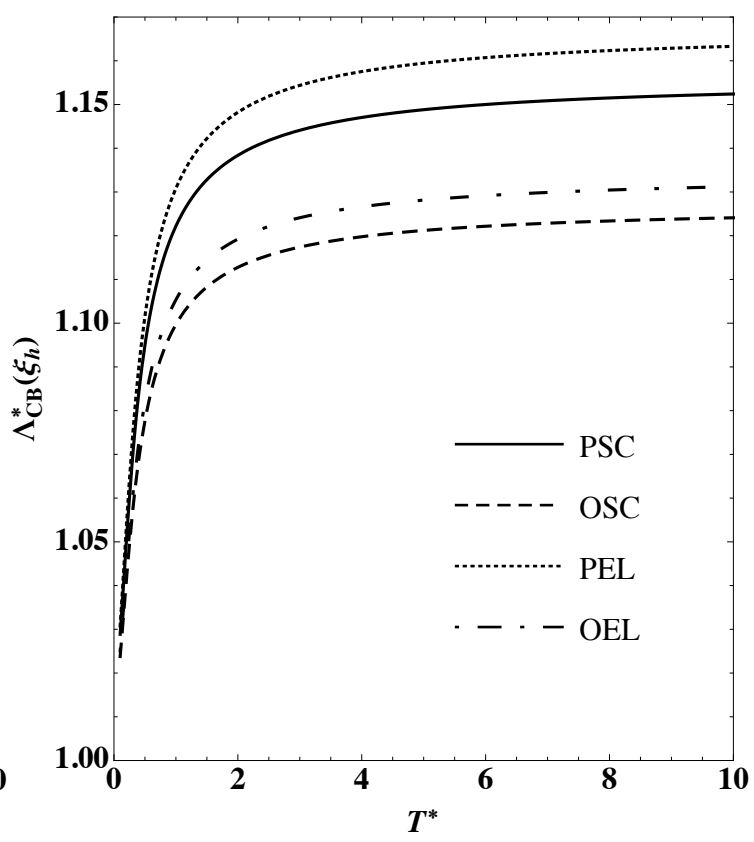

(b)

Figura 5.6: Dependencia de los volúmenes de colisión con respecto a la temperatura. La elongación se mantiene fija: $\xi_{h}=2.0$.

\section{Segundo coeficiente virial}

El segundo coeficiente virial CANC reducido se obtiene combinando las ecuaciones $(5.29)$ y $(5.30$ con la ecuación (3.13 pág. 33). Dos ejemplos de este coeficiente son mostrados en las figuras 5.7(a) y 5.7(b) para cuerpos convexos elipsoidales (prolato y oblato, respectivamente); para cuerpos esferocilíndricos el comportamiento es muy similar.

\section{Suavidad repulsiva y atractiva}

La función suavidad (repulsiva y atractiva) tiene una dependencia única con la elongación de la partícula. Para los cuerpos convexos empleados prolato y oblato, tanto la suavidad repulsiva como para la atractiva son obtenidas sustituyendo las cantidades fundamentales respectivas en las ecuaciones $(5.32)$ y $(5.33$ y para el caso 


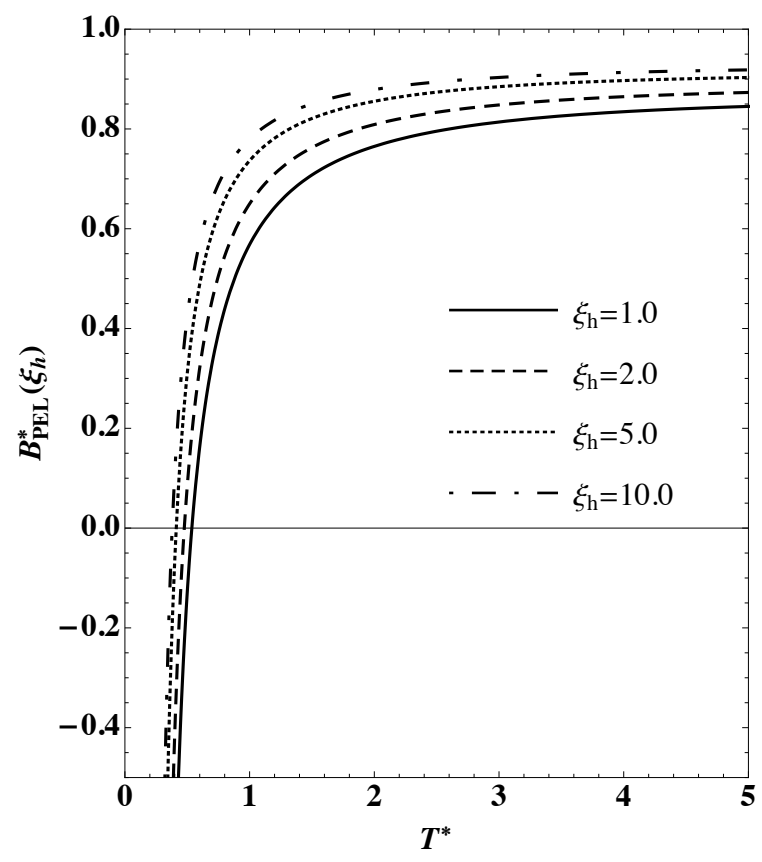

(a)

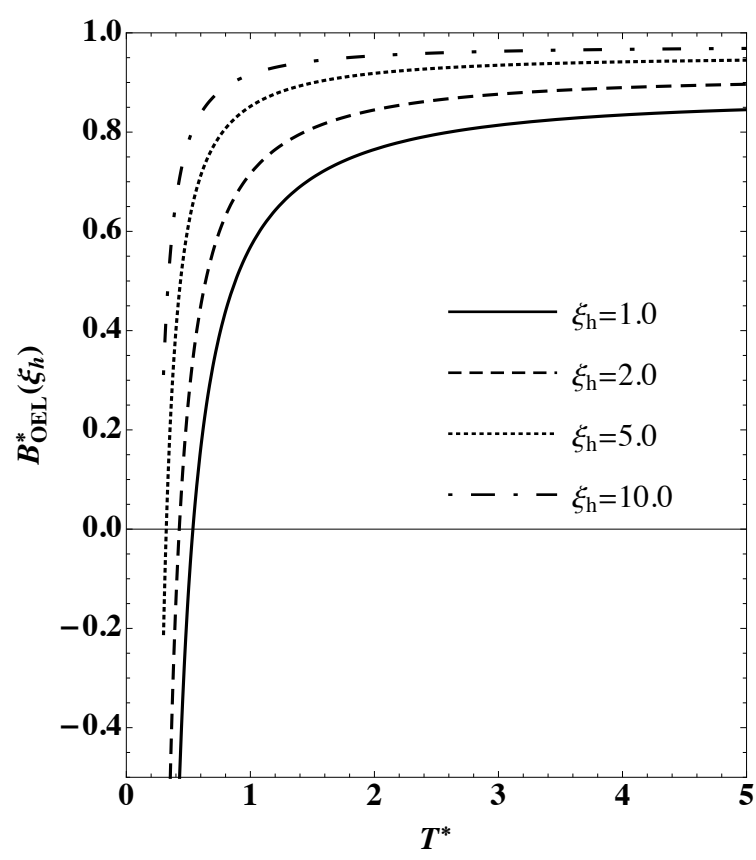

(b)

Figura 5.7: Segundos coeficientes viriales para interacciones CANC, PEL (a), OEL (b), para distintas elongaciones.

$\lambda_{0}=3 / 2$ se muestran algunos ejemplos en las figuras 5.8(a) y $5.8(\mathrm{~b})$ para los esferocilindros prolato y oblato, respectivamente.

\subsection{Aplicación a sistemas reales}

En las secciones anteriores se han introducido modelos ANC generales para el estudio de moléculas convexas y se ha revisado el efecto de la elongación sobre el segundo coeficiente virial. En esta sección se aplicarán dichos resultados al estudio de fluidos reales; en específico, con moléculas consideradas lineales tales como los $n$ alcanos (nA) y los n-perfluoroalcanos (nPA). Las características "geométricas" de estas moléculas se considerarán similares a las de un cuerpo convexo del tipo esferocilíndrico prolato, por lo que la elongación de dicho modelo puede vincularse al número de carbonos de la cadena principal de la molécula real. Establecido este vínculo se construye un modelo ANC que reproduzca la termodinámica de los gases de dichas moléculas.

Empleando el modelo ANC, Ramos et. al. [37] realizaron la inversión de datos experimentales del segundo coeficiente virial de las familias nA y $\mathrm{nPA}$, obteniendo parámetros ANC $(\epsilon, \delta, S)$ para cada uno de los primeros elementos de ambas familias y cuyos suavidades se hallan en la tabla 5.2. En particular, el cambio de la suavidad en términos del número de carbonos de la cadena principal de cada elemento de una familia, es similar al que se presenta en la figura 5.8 es decir, al de la suavidad del sistema de cuerpos convexos con respecto a la elongación de los mismos. En general, se busca una relación directa entre los datos experimentales del segundo coeficiente virial de la sustancia real con los parámetros efectivos de la teoría ANC.

La teoría ANC relaciona linealmente al segundo coeficiente virial ANC con uno de referencia, ec. [3.26), donde la suavidad $S$ es precisamente la pendiente de dicha función. Para el caso de cuerpos convexos, y en específico para el PSC, este comportamiento lineal se visualiza en las gráficas de la figura 5.2. La función suavidad que relaciona ambas cantidades puede elegirse igual a la forma funcional de las suavidades $S_{\mathrm{R}}$ ó $S_{\mathrm{A}}$, 


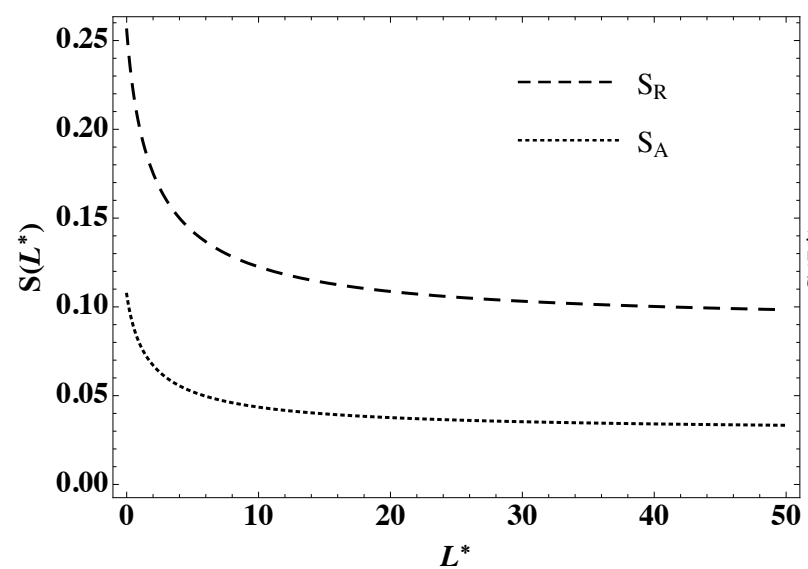

(a)

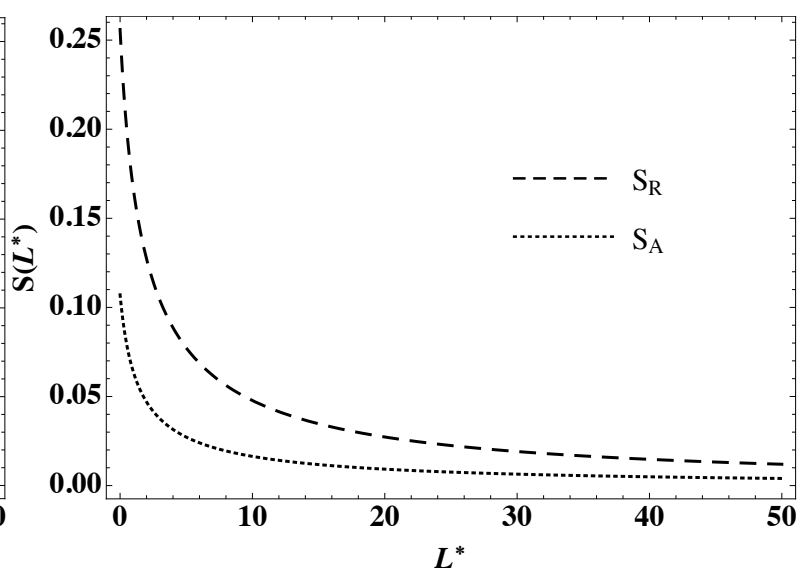

(b)

Figura 5.8: Suavidades repulsiva $S_{\mathrm{R}}\left(L^{*}\right)$, atractiva $S_{\mathrm{A}}\left(L^{*}\right)$ para una interacción PSC y para una OSC.

\begin{tabular}{|c|c|c|c|c|c|c|c|c|}
\hline Familia & $\mathbf{1}$ & $\mathbf{2}$ & $\mathbf{3}$ & $\mathbf{4}$ & $\mathbf{5}$ & $\mathbf{6}$ & $\mathbf{7}$ & $\mathbf{8}$ \\
\hline $\mathbf{n A}$ & 0.9073 & 0.8088 & 0.7008 & 0.6148 & 0.5503 & 0.5119 & 0.4693 & 0.4388 \\
\hline nPA & 0.6558 & 0.5742 & 0.4943 & 0.4382 & 0.3946 & 0.3584 & 0.3294 & \\
\hline
\end{tabular}

Tabla 5.2: Suavidades para la familia de $n$-alcanos y $n$-perfluoroalcanos obtenidas por del Río et. al. [37] mediante la inversión de datos experimentales del segundo coeficiente virial. 
es decir,

$$
S\left(\xi_{h}\right)=\frac{1}{b_{h}^{*}\left(\xi_{h}\right)}\left[\frac{A_{h}+\frac{M_{h}^{2}}{4 \pi}}{\frac{2 \pi}{3} h^{2}}\left(\frac{\lambda_{0}-1}{2}\right) \eta_{1}+\frac{M_{h}}{\frac{2 \pi}{3} h}\left(\frac{\lambda_{0}-1}{2}\right)^{2} \eta_{2}+\left(\frac{\lambda_{0}-1}{2}\right)^{3}\right]
$$

donde ahora $K, \eta_{1}, \eta_{2}$ y $\lambda_{0}$ son parámetros libres. Si además se supone una dependencia lineal de la elongación del cuerpo convexo que modela la molécula con el número de carbonos $n$, se tiene para el caso del esferocilindro prolato que

$$
L^{*}=(n-1) K
$$

donde $L^{*}=\xi_{h}-1$ y la constante de proporcionalidad $K$ mide la elongación de una fracción de la cadena correspondiente a un carbono.

Los mejores parámetros del modelo anterior que ajustan los datos de suavidad de la tabla 5.2 aparecen en la tabla 5.3 y sus respectivas curvas en la figura 5.9. En dicho ajuste no se consideraron los primeros elementos de cada familia $\left(\mathrm{CH}_{4}\right.$ y $\left.\mathrm{CF}_{4}\right)$ debido a su ligera desviación cualitativa con respecto a los otros elementos. Sin embargo, la forma en la que esta escrita la ecuación (5.38) permite extrapolar la suavidad para el caso de la elongación nula $(n=1)$. Para el caso de los alcanos la suavidad extrapolada es $S=1.0$, el que supera ligeramente al del metano $(S=0.9073)$; mientras que para los nPA es de $S=0.738$, superando de la misma manera a la sustancia $\mathrm{CF}_{4}(S=0.6558)$ - ver tabla 5.2

\begin{tabular}{ccccc}
\hline Familia & $\lambda_{0}$ & $\eta_{1}$ & $\eta_{2}$ & $K$ \\
\hline $\mathrm{nA}$ & 1.742 & 0.453 & 11.129 & 0.456 \\
$\mathrm{nPA}$ & 1.312 & 1.328 & 22.641 & 0.452 \\
\hline
\end{tabular}

Tabla 5.3: Parámetros ajustados de las suavidades en la tabla 5.2 según el modelo 5.38.

La constante de proporcionalidad para los $n$-alcanos obtenida en este trabajo ( $K=0.456)$ es muy similar a la longitud de enlace reducida $L^{*}=0.4123$ utilizada por Vega et. al. [29], en el estudio de coeficientes viriales de alcanos. Esta longitud es muy cercana a la razón entre la longitud de enlace C-C: $l=1.54 \AA$ y el parámetro de longitud en una interacción sitio-sitio Lennard-Jones: $d=3.70 \AA$ A Para el caso nPA, la constante de proporcionalidad obtenida es muy distinta a la de Ramos et. al. [37], mientras que en este trabajo es de $K=0.452$, lo que indica un tamaño reducido por carbono muy similar al de los alcanos; el obtenido en dicha referencia es mucho menor, $K=0.2$. Es decir, aunque los tamaños absolutos para ambas moléculas sean distintas, debido al cambio de los átomos de hidrógeno por fluóres, la elongación de moléculas de distintas familias con número igual de carbonos será muy similar.

\subsection{Conclusiones}

Este capítulo se ha centrado en la construcción de un potencial efectivo del tipo ANC que reproduce la termodinámica de un gas formado por moléculas convexas. El capítulo inicia estableciendo una relación directa entre la visión ANC y el trabajo desarrollado por otros autores al modelar a una molécula mediante un cuerpo convexo. Para ello se ha empleado un potencial CSW que es una extensión al de un pozo cuadrado pero con una dependencia en la orientación angular relativa entre las moléculas; el límite impenetrable consiste en un CB y el alcance del pozo corresponde también a un cuerpo convexo paralelo al central. Utilizando este nuevo potencial se obtienen formas analíticas para el segundo coeficiente virial y la temperatura de Boyle, escritas en función de las medidas fundamentales del CB empleado. 


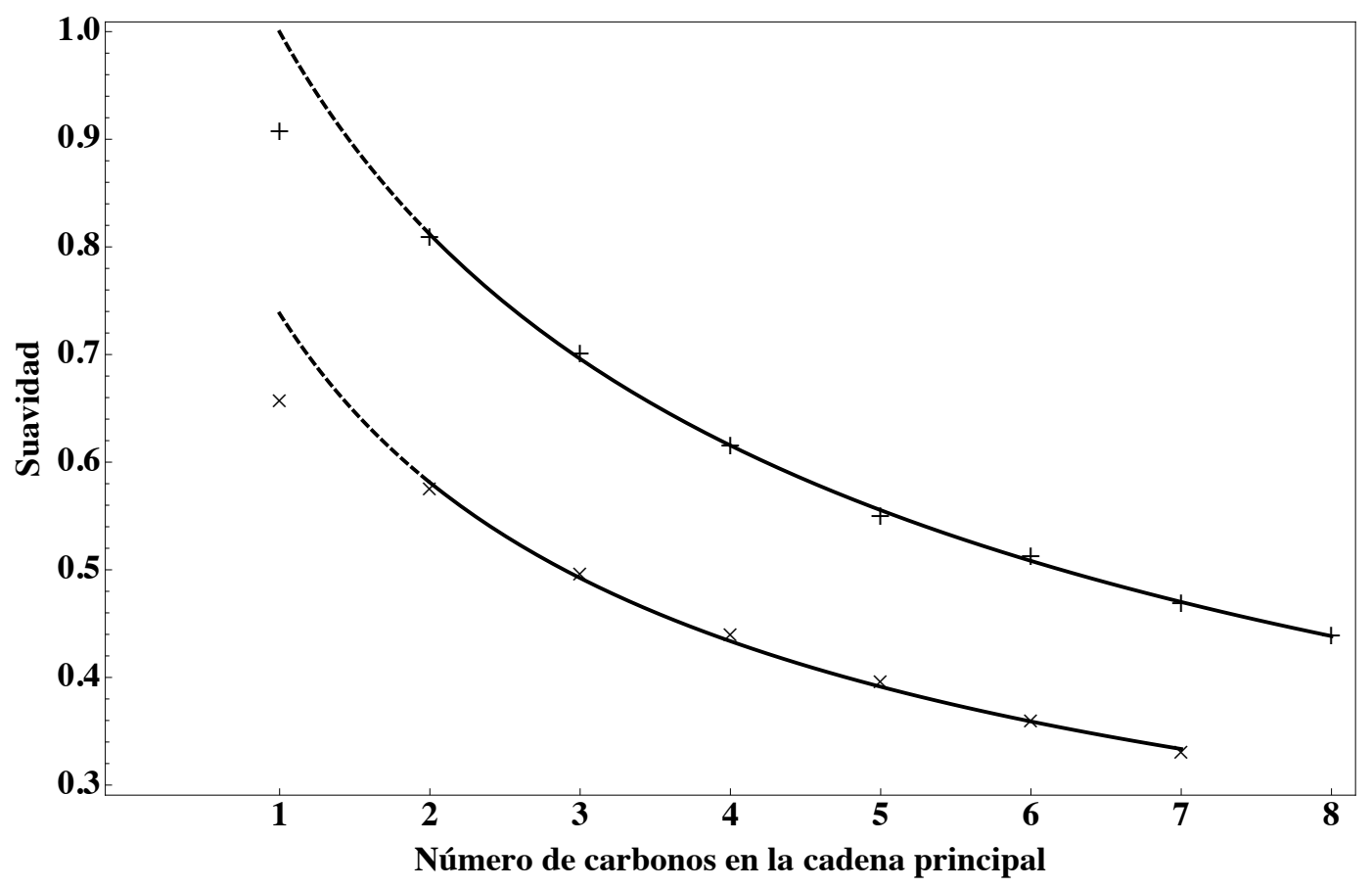

Figura 5.9: Dependencia de la suavidad con respecto al número de carbonos de la molécula usando un cuerpo PSC. Las curvas sólidas corresponden al ajuste hecho a la ecuación (5.38) empleando los datos experimentales dados por Ramos et. al. [37]: $\mathrm{nA}(+), \mathrm{nPA}(\times)$.

En esta parte del trabajo se ha considerado un conjunto amplio de cuerpos convexos: esferocilindros (prolato y oblato), elipsoides (prolato y oblato), esfero-cono, doble-cono, tetraedro regular y octaedro regular, presentando todos ellos un comportamiento similar cuando la elongación se modifica. El comportamiento del segundo coeficiente virial $B_{\mathrm{CSW}}^{*}$ con respecto a la elongación de la molécula es presentado en la figura 5.4 . En dicha figura se muestra la dependencia de este coeficiente con respecto a la temperatura para distintos valores de $\xi$ y de nuevo para distintos CB. En todos los casos se observa que cuando el cuerpo convexo se aleja de la esfericidad comienza a cobrar mayor relevancia la parte atractiva del coeficiente, es decir, el término negativo de la ecuación (3.13). El resultado anterior se verifica más fácilmente observando el comportamiento de la temperatura de Boyle, $T_{\mathrm{B}}^{*}$. Esta temperatura corresponde a aquella en la que el segundo coeficiente virial se hace cero, por lo que al darse el efecto arriba descrito la intersección con el eje de la temperatura se corre hacia la izquierda dando como resultado la disminución mostrada en la figura 5.5. El conjunto de resultados presentados concuerdan con trabajos de otros autores, mostrando que la elongación efectiva $\xi$ de la molécula influye en el ancho del potencial efectivo haciéndolo más atractivo cuando el cuerpo se elonga. Al final de la sección 5.3. se hace referencia al cálculo del segundo coeficiente virial de cuerpos convexos, realizado por Kihara, que interactúan mediante un potencial de pozo cuadrado (ligeramente distinto al aquí encontrado) y en dicha sección se ha analizado su equivalencia con el coeficiente virial obtenido.

El siguiente paso consiste en un replanteamiento del modelo anterior el cual permite obtener formas analíticas de los volúmenes de colisión y escribirlos en términos de un potencial continuo (radialmente simétrico) y con una dependencia explícita con la distancia entre los nódulos centrales $\rho$ de los cuerpos convexos. El potencial del que se parte es, específicamente, una función discreta en la distancia $r$ (la distancia entre centros de masa de los cuerpos convexos) y en función de la orientación angular, ec. (5.13. Esta dependencia permite que la energía de interacción entre dos moléculas convexas se mantenga constante mientras la distancia entre sus nódulos centrales se encuentre fija; dicha característica es la que permite escribir de forma analítica las cantidades físicas 
calculadas y en términos del covolumen formado por los dos cuerpos interactuantes. Así, los volúmenes de colisión (al igual que el segundo coeficiente virial) logran ser expresados en términos de funciones gamma incompletas con una dependencia explícita en la temperatura y en las medidas fundamentales del cuerpo convexo utilizado; expresiones totalmente analíticas.

El potencial continuo incorporado en las expresiones anteriores ha sido del tipo ANC por lo que, lo mismo que en el caso polar, los parámetros efectivos incorporan las características de la molécula modelo. Los parámetros de suavidad y diámetro efectivos son escritos en función de las medidas fundamentales del CB empleado; caso especial se da para el parámetro efectivo de la energía el cual, como ya se ha establecido, por sus características especiales se mantendrá independiente de la elongación del cuerpo convexo y del estado del sistema.

El trabajo se concentró en aquellos cuerpos convexos cuyo límite en la elongación es la unidad correspondiente a una esfera: esferocilindros y elipsoides, ambos del tipo prolato y oblato. En las figuras 5.6 se observa el comportamiento de los volúmenes de colisión en términos de la temperatura reducida y de la elongación $\xi$ del cuerpo convexo. Como puede observarse, los resultados son similares al caso CSW: a medida que la elongación del $\mathrm{CB}$ aumenta el potencial efectivo del que parten dichas expresiones se hace más atractivo. La comparación entre los volúmenes de colisión con sus respectivos volúmenes de referencia (aquellos generados a la distancia $\rho=\rho_{0}$ del mínimo del potencial) permite la obtención de las funciones suavidad, $S_{\mathrm{R}}$ y $S_{\mathrm{A}}$, para las zonas repulsiva y atractiva del potencial, respectivamente. Ambas suavidades para el caso prolato y oblato del esferocilindro se muestran en la figura 5.8 ahí se visualiza de mejor manera el comportamiento del potencial efectivo con respecto a $\xi$ : las suavidades en ambas ramas disminuyen rápidamente a medida que el cuerpo se elonga mostrando que el potencial efectivo se hace más suave. Obsérvese en la misma figura que para elongaciones grandes la suavidad tiende a un valor constante y el mismo comportamiento se halla para las suavidades en el caso oblato, Fig. 5.8(b). aunque dichos parámetros caen mucho más rápido con $\xi$ en comparación al caso prolato y para elongaciones grandes sus valores son cercanos al cero.

El segundo coeficiente virial para el gas formado por las moléculas convexas se presenta en la sección 5.5.2. figuras 5.7(a) y 5.7(b) de la página 91 , en dichas figuras se muestra el comportamiento de este coeficiente con respecto a la temperatura reducida y para distintas elongaciones de los cuerpos PSC y OSC, respectivamente. Los resultados son muy similares a los hallados en el caso CSW aunque en este caso se tiene en $S_{\mathrm{R}}$ y en $S_{\mathrm{A}}$ dos parámetros que miden numéricamente cuánto se suaviza el potencial efectivo involucrado. El cálculo del segundo coeficiente virial para otros cuerpos convexos (por ejemplo, el resto de cuerpos mencionados en la tabla C.1 ó aquellos que aparecen en las referencias en el capítulo (4) puede hacerse de forma directa sustituyendo sus respectivos volúmenes de colisión en la expresión dada por la ec. (3.3); es decir, el método puede aplicarse, en principio, a cualquier $\mathrm{CB}$ bien caracterizado.

Cuando se utiliza el potencial LJ 12-6 en las expresiones 5.17) y 5.18, y haciendo uso de las ecuaciones (3.11), 3.12) y (3.13), resulta fácil demostrar que el segundo coeficiente virial es el mismo que se halla en la teoría ANC en su versión modificada, ec. 3.51), sección 3.5.4 El desarrollo empleado para obtener los parámetros $S_{\mathrm{R}}$ y $S_{\mathrm{A}}$ del potencial efectivo para estos sistemas nos ubica en la versión ANC $2 \mathrm{~S}$.

La aplicación de estos resultados a sustancias reales se presenta en la sección 5.6. En ella se plantea una equivalencia entre la elongación del cuerpo convexo con la de la molécula real. Se han elegido dos familias de sustancias que por sus características permiten vincular el tamaño de sus cadenas principales con la elongación del cuerpo convexo que lo representa: los $n$-alcanos y los n-perfluoroalcanos; así, la elongación del cuerpo convexo se puede escribir en función del número de carbonos de las moléculas. Los parámetros ANC 1S para cada uno de los integrantes de estas dos familias de sustancias existen en la literatura y el comportamiento del parámetro suavidad con respecto al número de carbonos de la cadena principal es muy similar al que realiza la función suavidad del modelo generado con respecto a la elongación, figura 5.8

Los cuerpos prolato han sido empleados en diversos trabajos de esta índole y aquí, en particular, se ha empleado el esferocilindro ya que con él se tiene la ventaja de obtener expresiones analíticas mucho más sencillas. La forma funcional de la suavidad se ha elegido similar al de las suavidades repulsiva y atractiva 
encontradas en el caso 2S, y siendo ajustada a los datos de suavidad de las dos familias. Una característica importante se rescata de estos resultados: El parámetro $K$ es muy parecido para los dos casos, lo cual significa que la elongación carbono-carbono es muy similar para las dos familias. Esto no significa que los tamaños entre moléculas de distintas familias, pero con igual número de carbonos, sean iguales sino que la razón largo-ancho (carbono-carbono) de las moléculas es muy similar para ambas familias.

\section{Referencias}

[1] T. Boublík. Mol. Phys., 90:585, 1997.

[2] T. Boublík. Mol. Phys., 95:363, 1998.

[3] D. C. Williamson and F. del Río. J. Chem. Phys., 109(11):4675-4686, 1998.

[4] A. Cuetos, B. Martínez-Haya, and S. Lago. J. Chem. Phys. B, 109(28):13729-13736, 2005.

[5] T. Boublík and J. Winkelmann. Mol. Phys., 96(3):435-441, 1999.

[6] J. Janeček and T. Boublík. Phys. Chem. Chem. Phys., 5:2391-2397, 2003.

[7] J. Janeček and T. Boublík. Mol. Phys., 104(2):197-210, 2006.

[8] B. M. Ladanyi and D. Chandler. J. Chem. Phys., 62(11):4308, 1975.

[9] T. Boublík. Coll. Czech. Chem.Commun., 59:756-767, 1994.

[10] T. Kihara. Intermolecular Forces. John Wiley and Sons, New York, 1978.

[11] A. Isihara. J. Chem. Phys., 18:1446, 1950.

[12] A. Isihara and T. Hayashida. J. Phys. Soc. Japan, 6:40-45, 1950.

[13] A. Isihara and T. Hayashida. J. Phys. Soc. Japan, 6:46-50, 1950.

[14] T. Kihara. J. Phys. Soc. Japan, 6(5):289-296, 1951.

[15] T. Kihara. J. Phys. Soc. Japan, 8:686-687, 1953.

[16] T. Kihara. Rev. Modern Phys., 25(4):831-843, 1953.

[17] T. Kihara. Rev. Modern Phys., 27(4):412-423, 1955.

[18] T. Kihara and K. Miyoshi. J. Stat. Phys., 13(4), 1975.

[19] K Yamazaki and T. Kihara. J. Stat. Phys., 14(6):509-519, 1976.

[20] T. Boublík. Mol. Phys., 27:1415-1427, 1974.

[21] M. Sindelka and T. Boublík. Mol. Phys., 96(2):243-247, 1999.

[22] M. Sindelka and T. Boublík. Mol. Phys., 97(9):1035-1051, 1999.

[23] T. Boublík. J. Phys. Chem. B, 108:7424-7429, 2004.

[24] I. Nezbeda. Chem. Phys. Lett., 41(1):55-58, 1976.

[25] I. Nezbeda. Czech. J. Phys. B, 35:752-761, 1985. 
[26] C. Vega and S. Lago. J. Phys. Chem., 94(1):310-320, 1991.

[27] C. Vega and S. Lago. Chem. Phys. Lett., 185(5,6):516-521, 1991.

[28] C. Vega and S. Lago. J. Phys. Chem., 96:1895-1899, 1992.

[29] C. Vega, S. Lago, and B. Garzón. J. Chem. Phys., 100(3):2182-2190, 1994.

[30] M. S. Wertheim. Mol. Phys., 89(4):989-1004, 1996.

[31] M. S. Wertheim. Mol. Phys., 89(4):1005-1017, 1996.

[32] J. H. Dymond. J. Chem. Phys., 54(9):3675-3681, 1971.

[33] G. C. Maitland, M. Rigby, E. B. Smith, and W. A. Wakeham. Statistical Intermolecular Forces. Clarendon Press, Oxford, 1981.

[34] J. O. Hirschfelder, C. F. Curtiss, and R. F. Bird. Molecular Theory of gases and liquids. Wiley, 1954.

[35] D. Cook and J. S. Rowlinson. Proc. R. Soc. Lond. A, 219:405-418, 1953.

[36] J. E. Ramos, F. del Río, and I. A. McLure. J. Phys. Chem. B, 102:10576-10585, 1998.

[37] J. E. Ramos, F. del Río, and I. A. McLure. Phys. Chem. Chem. Phys., 2:2731-2741, 2000.

[38] C. G. Gray, K. E. Gubbins, and C. G. Joslin. Theory of Molecular Fluids Volume 2: Applications. Oxford University Press, Oxford, 2011. 


\section{Capítulo 6}

\section{Conclusiones y perspectivas}

\section{Conclusiones}

En esta tesis se han construido potenciales efectivos esféricos para dos fluidos cuyas partículas interactúan mediante un potencial anisotrópico: el primero formado por dipolos puntuales y el segundo por cuerpos convexos. Los sistemas efectivos son del tipo ANC con parámetros que pueden incluir una dependencia explícita del estado y de las propiedades moleculares intrínsecas del fluido estudiado; así, en el primer caso, dependerán de la intensidad del dipolo de la partícula y, para el segundo, de la forma geométrica de la misma. El análisis de este tipo de fluidos suele ser complejo y generalmente se realiza vía teoría de perturbaciones o mediante simulación computacional, por lo que estas interacciones efectivas aportan una herramienta simplificada con la cual estudiarlos, además de generar una comprensión más amplia de la física del fluido.

Para ambos fluidos se han obtenido expresiones explícitas del segundo coeficiente virial $(B)$, al igual que de la temperatura de Boyle $\left(T_{\mathrm{B}}\right)$, y sólo en el caso de los fluidos polares se ha trabajado con densidades mayores a la crítica, planteándose incluso una ecuación de estado (EDE) para fluidos Stockmayer (SM) polarizables (SMP).

Aunque el concepto de potencial efectivo es el mismo para ambos sistemas, el hecho de usar diámetros de colisión en la teoría ANC (capítulo 3), sumado a la funcionalidad de los respectivos potenciales, ha hecho que nos inclinemos por metodologías distintas en su obtención. Así, para un fluido Stockmayer no se calculan de inicio los diámetros de colisión, sino que primero se realiza un promedio angular del potencial SMP, mientras que en el caso de los cuerpos convexos dichos diámetros sí son obtenidos de inmediato. Es importante notar que, aunque distintos los planteamientos de ambos potenciales efectivos, el objetivo final es alcanzado: obtener efectos promedio representativos, incluidos en una función potencial, que permitan reproducir propiedades termodinámicas de los sistemas de estudio.

El capítulo 4 se concentra en el estudio de fluidos polares cuyas partículas interactúan según el potencial SMP. Estas partículas consisten en un centro Lennard-Jones con un dipolo puntual incrustado y que están sujetas a la polarización, sección 4.4 En búsqueda de efectos promedio, una primera simplificación del potencial se ha logrado promediando angularmente al potencial SMP usando el marco del ensamble canónico, obteniendo con ello un potencial esfericalizado (SMPs), sección 4.4.2 Este nuevo potencial funciona muy bien a altas temperaturas, bajas densidades e intensidades pequeñas para el dipolo. El principio de estados correspondientes (PEC) ha sido fundamental en este capítulo; basándonos en dicho principio una segunda simplificación se ha logrado al introducir un potencial efectivo del tipo ANC que reproduzca la funcionalidad del potencial SMPs: si ambos potenciales son conformales dichos sistemas comparten la misma termodinámica, sección 4.4.5. Comprobado esto último para un conjunto de estados bien determinados, la propuesta principal del trabajo consiste en suponer que el sistema efectivo es una buena representación del sistema original SMP.

Este procedimiento entrega excelentes resultados a bajas densidades, como puede verse en la sección 4.5.3. 
en específico, en la reproducción del segundo coeficiente virial y de la temperatura de Boyle. Estos resultados dependerán de la intensidad del dipolo: a mayor momento dipolar más difícil es encontrar un potencial efectivo que sea conformal con el potencial SMPs. Así, se ha demostrado que para momentos dipolares pequeños y/o temperaturas grandes el potencial Lennard-Jones 12-6 funciona adecuadamente como un potencial efectivo, pero para condiciones distintas (en específico momentos dipolares mayores) un potencial ANC resulta una mejor elección.

Para densidades mayores, se incorpora un factor extra (denominado "efecto de frustración") en el parámetro energético del potencial efectivo, sección 4.5.4. Este efecto de largo alcance surge de introducir una nueva partícula en la interacción binaria de dos dipolos. Para su construcción se ha considerado, además del principio de estados correspondientes, el tercer coeficiente virial de un fluido SMP hallado en la literatura.

Una EDE efectiva para un fluido SMP se ha construido usando el factor de frustración anterior y asignando de forma efectiva toda la información del líquido a los primeros dos coeficientes viriales, sección 4.6. Para ello se ha empleado información extra hallada en trabajos independientes (simulaciones de sistemas SM) y la EDE correspondiente al fluido LJ 12-6. Así, según el PEC esta última EDE con parámetros efectivos reproducirá los resultados de un fluido SM; por ello a esta última expresión efectiva se le asigna el nombre de EDE de un fluido SM. Esta EDE entrega resultados adecuados para densidades por arriba de la crítica, temperaturas subcríticas y momentos dipolares iguales o menores a la unidad, correspondiente a las curvas líquido-vapor de un sistema SM; para momentos dipolares mayores la EDE se emplea de forma aproximada. Esta ecuación se ha usado para construir la curva ortobárica de sistemas SMP que concuerda con los resultados obtenidos por otros autores, sección 4.6.3. El efecto de polarizabilidad (obtenida a primer orden a nivel del segundo coeficiente virial) dentro del modelo puede aplicarse para fluidos SMP con momento dipolar pequeño y polarizabilidades altas.

En el capítulo 5 se han obtenido potenciales efectivos que reproducen la termodinámica básica de sistemas formados por cuerpos convexos (CB). Todo ello en términos de sus propiedades geométricas $V, A$ y $M$ conocidas como medidas fundamentales, las cuales son escritas, a su vez, en función de la elongación del cuerpo. El potencial efectivo se ha construido para dos interacciones entre CB's: el pozo cuadrado convexo (CSW) y el ANC convexo (CANC), que es una extensión continua del potencial inmediato anterior.

El potencial de pozo cuadrado convexo es una variante del pozo cuadrado ya estudiado por Kihara, aunque en este caso se establece una relación entre el segundo coeficiente virial de un fluido CSW con respecto al respectivo coeficiente CSW esférico tomado como referencia, sección 5.3.1. Se demuestra que la relación entre ambos coeficientes es lineal por lo que se ha introducido, a similitud de la teoría ANC, un parámetro suavidad dependiente de la elongación de la partícula, sección 5.3.4. El resultado anterior se extiende a un caso continuo obteniéndose de forma analítica los diámetros medios de colisión $\sigma$ y $R$ (sección 5.4 para cualquier cuerpo convexo y cuya combinación según la teoría ANC lleva al segundo coeficiente virial común en la termodinámica de cuerpos convexos. Nuevamente, al igual que el caso CSW, el segundo coeficiente virial de un fluido CANC cumple una relación lineal con el respectivo coeficiente virial de un fluido CANC esférico. Dicha relación lineal, escrita en la forma ANC, permite hallar la forma exacta de los parámetros de suavidad (repulsivo y atractivo) en términos de la elongación de la partícula.

Particularmente se ha trabajado con esferocilindros prolatos (por la sencillez funcional de sus medidas fundamentales) para modelar moléculas lineales, tales como los $n$-alcanos y $n$-perfluoroalcanos, sección 5.6. Se han obtenido expresiones para los parámetros efectivos de la suavidad repulsiva y atractiva para el potencial ANC. Estos parámetros son escritos en función del número de carbonos de la cadena principal de la molécula acorde a los resultados obtenidos por otros autores; sin embargo, el método no permite hallar el parámetro energético ya que éste permanece constante.

Los resultados presentados en el capítulo 4 (Sistemas Polares) han sido publicados en la referencia [42]:

Rocha-Ichante A., del Río F., Ávalos E., "Equation of state and liquid-vapor equilibrium of polarizable Stockmayer fluids", J. Chem. Phys., 133, 2010.

Por último, paralelo a este trabajo, se han encontrado expresiones analíticas para los volúmenes de colisión 
de referencia ANC. Los volúmenes $b_{0}^{*}\left(T^{*}\right)$ y $\Lambda_{0}^{*}\left(T^{*}\right)$ (en sus versiones $2 \mathrm{~S}$ original y modificado) son escritas en términos de funciones gamma incompletas. Estas nuevas formas analíticas se han empleado en los capítulos 4 y 5 logrando expresiones matemáticas más compactas, y rutinas computacionales mucho más sencillas. Estos hechos, y los recientes trabajos en proceso, muestran que la teoría ANC es un excelente modelo efectivo para trabajar este tipo de fluidos.

\section{Perspectivas}

En cuanto a sistemas efectivos polares. La construcción de un modelo efectivo ANC, que reproduce la termodinámica de sistemas SM y SMP, se ha hecho vía el principio de estados correspondientes. Esto significa que existe conformalidad entre nuestro potencial modelo y un potencial SM promediado sobre todas las posibles configuraciones de los dipolos, es decir, un potencial esfericalizado. Para magnitudes pequeñas del dipolo la conformalidad se logra directamente con un potencial LJ 12-6; sin embargo, cuando dicha magnitud es mayor, el potencial ANC modificado ha sido requerido. El parámetro suavidad de nuestro modelo es el que permite que la conformalidad entre ambos potenciales se mantenga, aún para sistemas con dipolo grande. Un primer intento se ha realizado con este trabajo y es aquí, precisamente, donde quedan dos grandes objetivos pendientes: i) formalizar el modelo efectivo ANC modificado e ii) introducir el modelo ANC original para un estudio cuantitativo de este tipo de sistemas. Intentos en esta última dirección ya han sido realizados, como ya se mencionó en el cuerpo principal de la tesis; sin embargo, sólo se han logrado para intensidades bajas del dipolo.

Un punto importante revisado en este trabajo ha sido el efecto de frustración dentro de los modelos efectivos LJ y ANC. Este factor es un intento por interpretar cómo la interacción (de largo alcance) entre dos partículas SMP se ve afectada por la presencia de una tercera. Este factor reduce el parámetro efectivo de energía (la profundidad del pozo del potencial efectivo), y su efecto a bajas densidades aparece a nivel del tercer coeficiente virial. Un análisis desde el punto de vista de potenciales efectivos deberá incorporar más elementos que lo sustenten, por ejemplo, vía simulación molecular ó métodos perturbativos, especialmente a densidades grandes. Sin embargo, el hecho de que un potencial tipo ANC reproduzca el segundo coeficiente virial de fluidos SMP (aún para momentos dipolares considerados grandes) indica que sus parámetros efectivos incorporan adecuadamente las características del fluido polar, lo que da un peso importante al uso de este tipo de potenciales. Su aplicación a sistemas de mayor densidad está sujeto al conocimiento de una ecuación de estado de sistemas ANC, lo que hasta el momento sólo se tiene parcialmente.

Finalmente, como se ha mencionado en la sección 4.3 ya que los fluidos SMP son ampliamente usados en aquellos sistemas que mantienen una equivalencia con los polares (tales como los fluidos ferroeléctricos, magnetoreológicos y en el autoensamblaje en polímeros), se abre la posibilidad de introducir un modelo efectivo ANC, similar al construido en este trabajo, que intente reproducir las características principales de todos esos sistemas equivalentes.

En cuanto a sistemas formados por cuerpos convexos. Para los modelos efectivos de pozo cuadrado y ANC, hasta el momento se tienen expresiones analíticas para los volúmenes de colisión, el segundo coeficiente virial y los parámetros de suavidad ANC, todas ellas en términos de las características de la elongación de la molécula y demás características geométricas. En principio, cualquier cuerpo convexo cuyas medidas fundamentales sean conocidas puede emplearse, por lo que su aplicación a fluidos reales puede realizarse considerando la geometría aproximada de la molécula, y cuyo tamaño relativo puede relacionarse con la elongación del cuerpo convexo empleado en dicho desarrollo. 
Apéndices 



\section{Apéndice A}

\section{Conceptos extras de Física Estadística}

\section{A.1. Integrales de configuración}

Trabajando en el ensemble gran canónico, el factor de compresibilidad está definido mediante la relación $\eta^{\prod}$

$$
\frac{P V}{k_{\mathrm{B}} T}=\ln \Xi .
$$

donde $\Xi$ es la función de partición del gran canónico. Para fluidos puros esta última función está dada por

$$
\Xi=\sum_{N=0} Q_{N}(N, V, T) e^{N \nu / k_{\mathrm{B}} T}
$$

con $Q_{N}$ como la función de partición canónica de un sistema de $N$ moléculas y $\nu$ el potencial químico por molécula del fluido.

La evaluación de la función de partición canónica se encuentra sujeta a la energía del sistema termodinámico. Si dicho sistema está compuesto de partículas identificables e interactuantes, su energía en el $i$-ésimo estado cuántico puede expresarse como

$$
E_{i}(N, V, T)=E_{i}^{*}+\Psi\left(\boldsymbol{r}_{1}, \boldsymbol{r}_{2}, \cdots, \boldsymbol{r}_{N}\right)
$$

donde el primer sumando identifica a la energía correspondiente para un conjunto hipotético de partículas independientes y $\Psi$ denota a la energía de configuración de las $N$ partículas a nivel macroscópico. Sustituyendo esta energía en la definición de la función de partición canónica se obtiene

$$
Q_{N}(N, V, T)=\sum_{i} \exp \left(-\frac{E_{i}}{k_{B} T}\right)=\sum_{i} \exp \left(-\frac{E_{i}^{*}}{k_{\mathrm{B}} T}\right) \cdot \frac{1}{V^{N}} \int \cdots \int \mathrm{e}^{-\Psi / k_{\mathrm{B}} T} d \boldsymbol{r}_{1} \boldsymbol{r}_{2} \cdots \boldsymbol{r}_{N}
$$

por lo que la ec. A.1 es escrita como

$$
\frac{P V}{k_{\mathrm{B}} T}=\ln \left[1+\sum_{N=1}^{\infty} Q_{N}(N, V, T) \lambda^{N}\right]
$$

y $\lambda=\exp \left[\nu / k_{\mathrm{B}} T\right]$. En el caso de moléculas monoatómicas la función de partición canónica contiene tan solo contribuciones traslacionales y A.2 puede ser escrito como

$$
\Xi=\sum_{N \geq 0} \frac{\Lambda^{-3 N}}{N !} Q_{N}^{C}(N, V, T) \mathrm{e}^{N \nu / k_{\mathrm{B}} T}
$$

\footnotetext{
${ }^{1}$ Ver referencias $[1-3]$.
} 
con la función $\Lambda$ definida por

$$
\Lambda=\sqrt{\frac{h^{2}}{2 \pi m k_{\mathrm{B}} T}}
$$

donde $m$ es la masa de la molécula y $h$ la constante de Planck.

El término $Q_{N}^{C}$ es la función de partición configuracional

$$
Q_{N}^{C}=\int \cdots \int \mathrm{e}^{-\Phi\left(\boldsymbol{r}_{1}, \boldsymbol{r}_{2}, \cdots, \boldsymbol{r}_{N}\right) / k_{\mathrm{B}} T} d \boldsymbol{r}^{N}
$$

para un sistema puro formado por $N$ moléculas monoatómicas en el volumen $V$.

Si hace un desarrollo del factor de compresibilidad en términos de la actividad absoluta $\left(a=\Lambda^{-3} \mathrm{e}^{\nu / k_{\mathrm{B}} T}\right) \mathrm{se}$ obtiene, la ecuación A.1 toma la forma

$$
\frac{p V}{k_{\mathrm{B}} T}=V \sum_{i \geq 1} b_{i} a^{i}
$$

el cual puede ser comparado con la expresión mostrada en A.6 al desarrollar el exponencial del factor de compresibilidad A.1 en la siguiente forma

$$
\mathrm{e}^{p V / k_{\mathrm{B}} T}=\mathrm{e}^{V \sum_{i \geq 1} b_{i} a^{i}}=\prod_{i \geq 1} \mathrm{e}^{V b_{i} a^{i}}=\prod_{i \geq 1} \sum_{m_{i} !}\left(V b_{i} a^{i}\right)^{m_{i}}
$$

y comparando los respectivos coeficientes de este último desarrollo con aquellos de la ecuación A.6 para igual número de moléculas se encuentra que

$$
\begin{aligned}
Q_{1}^{C} & =V b_{1} \\
Q_{2}^{C} & =\left(V b_{1}\right)^{2}+2 V b_{2} \\
Q_{3}^{C} & =\left(V b_{1}\right)^{3}+6 V^{2} b_{1} b_{2}+6 V b_{3} \\
\ldots &
\end{aligned}
$$

lo que indica que los coeficientes $b_{i}$ pueden expresarse así en términos de las integrales de configuración a partir de 1 hasta las $N$ molećulas de tal forma que se encuentren solas en el volumen total del sistema, es decir, a densidad cero. Tales arreglos son conocidos como racimos moleculares y por ello a los términos $b_{i}$ se les llama integrales de racimo.

Una forma de eliminar la actividad absoluta $a$ del procedimiento anterior es encontrar una relación entre este parámetro y la densidad $\rho$. Para tal efecto se parte del número de moléculas que existen en el gran canónico,

$$
N=k_{\mathrm{B}} T\left(\frac{\partial \ln \Xi}{\partial \nu}\right)_{T, V}=\left(\frac{\partial \ln \Xi}{\partial \ln a}\right)_{T, V}
$$

y reordenando se logra una relación entre los dos parámetros de interés

$$
\rho=\frac{a}{V}\left(\frac{\partial\left(p V / k_{\mathrm{B}} T\right)}{\partial a}\right)_{T, V}=\sum_{i \geq 1} i b_{i} a^{i}
$$

lo que permite expresar a la actividad absoluta en términos de la densidad como

$$
a=\sum_{j \geq 1} c_{j} n^{j}
$$

cuya comparación con los coeficientes de la ecuación A.2 se tiene

$$
\begin{aligned}
& c_{1}=1 / b_{1} \\
& c_{2}=-2 b_{2} / b_{1}^{3} \\
& c_{3}=-3 b_{3} / b_{1}^{4}+8 b_{2}^{2} / b_{1}^{5} \\
& \ldots
\end{aligned}
$$


por lo que la ecuación virial se escribe finalmente como

$$
\begin{aligned}
Z & =\frac{P V}{N k_{\mathrm{B}} T}=\frac{V}{N} \sum_{i \geq 1} b_{i} a^{i} \\
& =1+\left(b_{2} / b_{1}-2 b_{2} / b_{1}^{2}\right) \rho+\left(8 b_{2}^{2} / b_{1}^{4}-3 b_{3} / b_{1}^{3}-4 b_{2}^{2} / b_{1}^{4}+b_{3} / b_{1}^{3}\right) \rho^{2} \cdots
\end{aligned}
$$

\section{A.2. Expresiones analíticas de $B(T)$ y $C(T)$ para sistemas específicos}

A continuación se presentan el segundo y tercer coeficiente virial analíticos (sección 2.3.2 pág. 13) para algunos potenciales de interés en esta investigación. Algunas de las integrales que aparecen en estos cálculos sirven de base para aquellas que aquí han surgido, en especial aquellas que involucran el potencial Lennard-Jones $12-6$, ec. 2.12 .

\section{A.2.1. Integrales $\mathcal{F}_{k}(T)$ y $\mathcal{H}_{n}(T)$ en la literatura}

\section{Formas integrales $\mathcal{F}_{k}(T)$}

Las funciones $\mathcal{F}_{k}(T)$ surgen en el estudio de cuerpos convexos y su forma integral está dada por

$$
\mathcal{F}_{k}(T)=\int_{0}^{\infty}\left(1-e^{-\Phi_{0}(x) / k_{\mathrm{B}} T}\right) d x^{k}
$$

la cual puede ser resuelta utilizando la técnica de Lennard-Jones $[4,5]$. En el caso que $\Phi_{0}=\Phi_{\mathrm{LJ}}$ se tiene

$$
\mathcal{F}_{k}(T)=-\frac{k}{12} \sum_{j=0}^{\infty} \frac{2^{j}}{j !} \Gamma\left(\frac{6 j-k}{12}\right)\left(\frac{\epsilon}{k_{\mathrm{B}} T}\right)^{\frac{6 j+k}{12}}
$$

que puede ser generalizada fácilmente para potenciales tipo Mie [6].

La integral A.17) puede ser resuelta de manera cerrada de diversas maneras [7-9] y expresarse en términos de funciones especiales como

$$
\begin{aligned}
\mathcal{F}_{k}(T)= & \left(\frac{2 \epsilon}{k_{\mathrm{B}} T}\right)^{\frac{6+k}{12}} \Gamma\left[\frac{6-k}{6}\right] \exp \left[\frac{\epsilon}{2 k_{\mathrm{B}} T}\right] \times \\
& \left\{\left(\frac{2 \epsilon}{k_{\mathrm{B}} T}\right)^{-\frac{1}{2}}\left(\frac{6-k}{6}\right) D_{-\frac{12-k}{6}}\left[-\left(\frac{2 \epsilon}{k_{\mathrm{B}} T}\right)^{\frac{1}{2}}\right]-D_{-\frac{6-k}{6}}\left[-\left(\frac{2 \epsilon}{k_{\mathrm{B}} T}\right)^{\frac{1}{2}}\right]\right\}
\end{aligned}
$$

en términos de funciones cilíndrico-parabólicas [10], $D_{y}[x]$. Una nueva presentación de $\mathcal{F}_{k}(T)$ ha sido obtenida durante la investigación y en la siguiente sección se muestran los principales cálculos.

\section{Formas integrales $\mathcal{H}_{n}(T)$}

La solución analítica $\mathcal{H}_{n}(T)$ fue introducida por Buckingham et. al. [11] para lograr mejores resultados (desde el punto de vista numérico) a los obtenidos originalmente por Stockmayer [12] y Rowlinson [13], en sus estudios de fluidos polares. La integral que involucra esta función está dada por

$$
\int_{0}^{\infty} r^{-n} \exp \left[-\frac{\Phi_{0}}{k_{\mathrm{B}} T}\right] r^{2} d r=\frac{1}{12} \sigma^{3-n}\left(\frac{k_{\mathrm{B}} T}{4 \epsilon}\right) \mathcal{H}_{n}(T)
$$

para el caso en que $\Phi_{0}=\Phi_{\mathrm{LJ}}$, y en las referencias citadas se demuestra que $\mathcal{H}_{n}(T)$ está dada por

$$
\mathcal{H}_{n}(T)=\left(\frac{4 \epsilon}{k_{\mathrm{B}} T}\right)^{\frac{15-n}{12}} \sum_{m=0}^{\infty} \frac{1}{m !}\left(\frac{4 \epsilon}{k_{\mathrm{B}} T}\right)^{m / 2} \Gamma\left[\frac{6 m+n-3}{12}\right] .
$$

Para el caso general, $\Phi_{0}=\Phi_{\text {Mie }}$, ec. 2.14, la integral A.20 se resuelve de manera similar siendo reportados por diversos autores [14]. 


\section{A.2.2. Segundo coeficiente virial}

\section{Esferas duras}

Para este potencial, ec. 2.10, la integración es directa

$$
B_{\mathrm{HS}}(T)=-\frac{1}{2} \int_{0}^{\sigma}(-) 4 \pi r^{2} d r=\frac{2 \pi \sigma^{3}}{3}
$$

el cual resulta independiente de la temperatura. El valor de este coeficiente es cuatro veces el volumen de una esfera con diámetro $\sigma$.

\section{Pozo cuadrado}

Para el potencial SW, ec. 2.11), el segundo coeficiente virial se escribe en la forma

$$
\begin{aligned}
B_{\mathrm{SW}}(T) & =2 \pi\left\{\int_{0}^{\sigma} r^{2} d r+\int_{\sigma}^{\lambda \sigma}\left(1-\mathrm{e}^{\epsilon / k_{\mathrm{B}} T}\right) r^{2} d r\right\} \\
& =b_{0}\left[1-\left(\lambda^{3}-1\right)\left(\mathrm{e}^{\epsilon / k_{\mathrm{B}} T}-1\right)\right]
\end{aligned}
$$

donde el volumen $b_{0}$ es el segundo coeficiente virial de la esfera dura, ec. A.22 y se tiende a este valor a medida que se cumple el límite $\lambda \rightarrow 1$ o $\epsilon \rightarrow 0$. El comportamiento de $B_{\mathrm{SW}}$ a temperaturas bajas e intermedias es (cualitativamente hablando) bastante cercano al que típicamente muestra el experimento de dicha propiedad, figura 2.4 , página 14 .

\section{Lennard-Jones}

Para el caso del potencial LJ, ec. 2.12, el segundo coeficiente virial en términos de $\sigma$ puede ser escrito en la siguiente forma [4]

$$
B_{\mathrm{LJ}}(T)=\frac{2 \pi}{3} \sigma^{3} \mathcal{F}_{3}(T)
$$

donde la integral $\mathcal{F}_{3}(T)$ forma parte de las ecuaciones A.18. Esto es, el segundo coeficiente virial en forma semianalítica es

$$
B_{\mathrm{LJ}}(T)=-\frac{\pi}{6} \sigma^{3} \sum_{j=0}^{\infty} \frac{2^{j}}{j !} \Gamma\left(\frac{2 j-1}{4}\right)\left(\frac{\epsilon}{k_{\mathrm{B}} T}\right)^{\frac{2 j+1}{4}}
$$

y en forma cerradd $2^{2}$

$$
\begin{aligned}
B_{\mathrm{LJ}}(T)= & \frac{\pi^{3 / 2}}{3 \sqrt[4]{2}} \sigma^{3}\left(\frac{\epsilon}{k_{\mathrm{B}} T}\right)^{1 / 4} \exp \left(\frac{\epsilon}{2 k_{\mathrm{B}} T}\right) \times \\
& \left\{D_{-\frac{3}{2}}\left[-\left(\frac{\epsilon}{2 k_{\mathrm{B}} T}\right)^{1 / 2}\right]-\left(\frac{8 \epsilon}{k_{\mathrm{B}} T}\right)^{\frac{1}{2}} D_{-\frac{1}{2}}\left[-\left(\frac{\epsilon}{2 k_{\mathrm{B}} T}\right)^{1 / 2}\right]\right\} .
\end{aligned}
$$

Cuando el potencial LJ elegido tiene la forma de la ec. 2.13, pág. 10) el respectivo segundo coeficiente virial resultante puede obtenerse sustituyendo simplemente el valor de $\sigma$ por $2^{-1 / 6} \delta$ en las ecuaciones A.25-A.27 3

\footnotetext{
${ }^{2} \mathrm{El}$ procedimiento completo puede revisarse en la referencia [9].

${ }^{3}$ Otra manera de escribir el segundo coeficiente virial del potencial LJ es el dado por la ecuación

$$
B_{\mathrm{LJ}}(T)=\frac{2 \pi}{3} \sigma^{3}\left[\mathcal{H}_{12}(T)-\frac{1}{2} \mathcal{H}_{6}(T)\right]
$$
}

con las funciones $\mathcal{H}_{n}(T)$ definidas por A.21, y utilizadas ampliamente en el caso de sistemas polares. 


\section{Kihara}

La dependencia angular, implícita en este potencial, hace que el cálculo del segundo coeficiente virial parta de la ecuación 2.20 pág. 2.20 . La geometría de cuerpos convexos (sección C.2 permite que la integración de esta ecuación sea analítica y liga el segundo coeficiente virial con el covolumen generado por dos de tales cuerpos. Sustituyendo el potencial de Kihara (2.15), el cual depende de la distancia más corta $\rho$ entre las superficies de dichos cuerpos, se puede demostrar que el segundo coeficiente virial de un sistema formado por moléculas convexas está dado por [15]

$$
2 B_{\mathrm{K}}(T)=\left\langle V_{h+h}\right\rangle+\int_{0}^{\infty}\left(1-\mathrm{e}^{-\Phi_{\mathrm{K}}(\rho) / k_{\mathrm{B}} T}\right)\left\langle A_{h+\rho+h}\right\rangle d \rho
$$

donde $h$ representa al nódulo de la molécula y el término general $\left\langle V_{h+\rho+h}\right\rangle$ puede ser escrito en términos de los parámetros del cuerpo convexo central $M_{h}, S_{h}$ y $V_{h}$ (ec C.10) permitiendo escribir finalmente al segundo coeficiente virial en la forma

$$
B_{\mathrm{K}}(T)=\frac{2 \pi}{3} \rho_{0}^{3} \mathcal{F}_{3}(T)+M_{h} \rho_{0}^{2} \mathcal{F}_{2}(T)+\left(A_{h}+\frac{M_{h}^{2}}{4 \pi}\right) \rho_{0} \mathcal{F}_{1}(T)+\left(V_{h}+\frac{M_{h} A_{h}}{4 \pi}\right)
$$

donde $\mathcal{F}_{k}(T)(k=1,2,3)$ son las integrales dadas en la sección A.2.1 La ecuación A.29 se reduce al caso LJ 12-6 A.24 cuando el volumen del cuerpo convexo central tiende a cero.

\section{Pozo cuadrado Kihara}

El caso más general de un potencial de pozo cuadrado convexo, en términos de la distancia más corta $\rho$ entre los nódulos duros, es escrito como

$$
\Phi(\rho)=\Phi_{\mathrm{SW}}(\rho)
$$

donde $\Phi_{\mathrm{SW}}$ es la interacción tipo pozo cuadrado convencional dada por la ecuación 2.11, pág. 10. El parámetro de distancia $\sigma$ se ha empleado para reducir las integrales $\mathcal{F}_{k}(T)$, (ec. A.17), obteniéndose

$$
\mathcal{F}_{k}\left(T^{*}\right)=1+\left(1-\mathrm{e}^{1 / T^{*}}\right)\left(\lambda^{k}-1\right)
$$

donde $\lambda=\rho / \sigma$, por lo que el cálculo del segundo coeficiente virial (ec. A.29, pág. 109 es directo. El caso particular de este potencial con $\lambda=2$ es resuelto por Kihara en las referencias [15, 16].

Cuando la parte impenetrable del potencial corresponde exactamente con el nódulo duro $(\sigma=0)$ se obtiene la versión del potencial CSW que aquí se ha manejado, ec. 5.1. pág. 81, ahora escrita en términos de la distancia mínima $\rho$ entre superficies de los CB

$$
\Phi_{\mathrm{CSW}}(\rho)=\left\{\begin{array}{cc}
\infty, & \rho>0, \\
-\epsilon, & 0<\rho<\rho_{0}, \\
0, & \rho_{0}<\rho,
\end{array}\right.
$$

donde $\rho_{0}$ es el ancho del pozo y la integrales arriba presentadas se reducen todas ellas a la forma simple $F_{k}=1-\mathrm{e}^{1 / T^{*}}$.

El segundo coeficiente virial obtenido mediante este camino tiene la forma

$$
B_{\mathrm{CSW}}\left(T^{*}, \xi_{h}\right)=\left(V+\frac{M A}{4 \pi}\right)-\left\{\left(A+\frac{M^{2}}{4 \pi}\right) \rho_{0}+M \rho_{0}^{2}+\frac{2 \pi}{3} \rho_{0}^{3}\right\}\left(\mathrm{e}^{1 / T^{*}}-1\right)
$$

que es el mismo dado en la expresión 5.5 en la página 81 . 


\section{Stockmayer}

A pesar de que el potencial SM tiene una forma analítica más compleja que las funciones LJ 12-6 o Kihara, la obtención del segundo coeficiente virial para este fluido se logra mediante un procedimiento muy semejante a los ya revisados. Esto es, se desarrolla el exponencial positivo del factor de Boltzmann y la integración se realiza sobre el término general dentro de la serie resultante.

El resultado final mediante este procedimiento puede ser escrito de diversas maneras [11-13] siendo todas ellas equivalentes. Una forma clásica de expresarlo es la siguiente [11]

$$
B_{\mathrm{SM}}(T)=B_{\mathrm{LJ}}(T)-\frac{\pi \sigma^{3}}{6} \sum_{n=1}^{\infty} 2^{n} t^{* 2 n}\left(\frac{4 \epsilon}{k_{\mathrm{B}} T}\right)^{2 n-1} \mathcal{H}_{n}(T)\left[\frac{n !}{(2 n+1) !}\right]^{2} \sum_{i=0}^{n} \frac{(2 i) !}{i !^{2}}
$$

donde

$$
t^{* 2}=\frac{\mu^{2}}{\sqrt{8} \epsilon \sigma^{3}}
$$

y $\mathcal{H}_{n}(T)$ está definida por la ecuación A.21. Como puede verse el término del lado derecho contiene al segundo coeficiente virial del potencial Lennard-Jones el cual está definido por cualquiera de las formas A.25- A.27.

\section{Stockmayer polarizable}

El segundo coeficiente virial para un fluido Stockmayer polarizable puede expresarse de la siguiente manera

$$
\begin{aligned}
B_{\mathrm{PSM}}\left(T, \mu^{2}, \alpha\right)= & B_{\mathrm{LJ}}(T)+\frac{x^{2}}{3} h_{1}+\frac{x^{4}}{25} h_{2}+\ldots \\
& +\left(2 x h_{1}+\frac{4 x^{3}}{5} h_{2}+\ldots\right) \alpha+O\left(\alpha^{2}\right)
\end{aligned}
$$

con $x=\mu^{2} / k_{\mathrm{B}} T$ y donde la integral

$$
h_{j}(T)=-2 \pi \int_{0}^{\infty} d r r^{2-6 j} \exp \left[-\frac{\Phi_{\mathrm{LJ}}}{k_{\mathrm{B}} T}\right]
$$

se resuelve numéricamente en [17] y en la misma referencia se hallan términos adicionales. En la siguiente sección se presenta una solución analítica para $h_{j}(T)$.

\section{A.2.3. Tercer coeficiente virial}

\section{Lennard-Jones}

Un procedimiento similar al cálculo del segundo coeficiente virial de este potencial es aplicado para la obtención de $C(T)$, dado por

$$
C_{\mathrm{LJ}}(T)=\frac{5}{9} \pi^{2} \sigma^{6}\left(\frac{\epsilon}{k_{\mathrm{B}} T}\right)^{1 / 2} \sum_{j=0}^{\infty} \gamma_{j}(12)\left(\frac{\epsilon}{k_{\mathrm{B}} T}\right)^{j / 2}
$$

El procedimiento completo puede revisarse en las referencias [6,18]; ahí mismo se presentan los cálculos numéricos de las integrales dobles $\gamma_{j}(12)$.

\section{Stockmayer}

El cálculo del tercer coeficiente virial para un fluido SM conlleva el mismo método utilizado para el coeficiente anterior y tiene la siguiente forma

$$
C_{\mathrm{SM}}(T)=C_{\mathrm{LJ}}(T)+\frac{2 \pi^{2}}{3} \sigma^{6} \sum_{n=2}^{\infty} \sum_{l=2}^{n} \frac{2^{n}}{n !\left(k_{\mathrm{B}} T\right)^{\frac{2 n+l+2}{4}}}\left(\begin{array}{l}
n \\
l
\end{array}\right)\left(\frac{\mu^{2}}{2 \sqrt{2} \epsilon \sigma^{3}}\right)^{l} Q_{n, l}
$$

donde los coeficientes numéricos $Q_{n, l}$ pueden ser consultados en la cita original [19]. Este coeficiente se escribe en términos del tercer coeficiente virial de un fluido LJ, $C_{\mathrm{LJ}}(T)$, ec. A.37). 


\section{Apéndice B}

\section{Integrales y ajustes numéricos extras.}

\section{B.1. Simplificación a la forma cerrada de $\mathcal{F}_{k}(T)$}

La expresión mostrada en A.19 puede simplificarse de sobremanera. Introduciendo el cambio de variable $\omega(T)=\frac{2 \epsilon}{k_{\mathrm{B}} T}$ y reacomodando los índices en $k$, la expresión se escribe como

$$
\mathcal{F}_{k}(T)=-\omega(T)^{\frac{k}{12}} \Gamma\left[\frac{6-k}{6}\right] \mathrm{e}^{\frac{\omega(T)}{4}}\left\{\left(\frac{k}{6}-1\right) D_{\frac{k}{6}-2}\left[-\omega(T)^{\frac{1}{2}}\right]+\omega(T)^{\frac{1}{2}} D_{\frac{k}{6}-1}\left[-\omega(T)^{\frac{1}{2}}\right]\right\}
$$

Ayudándonos de las definiciones $z=-\omega(T)^{1 / 2}, p=\frac{k}{6}-1$, y usando las fórmulas de recurrencia de las funciones cilíndrico-hiperbólicas [10,20], la expresión entre corchetes se reduce a

$$
\begin{aligned}
\left(\frac{k}{6}-1\right) D_{\frac{k}{6}-2}\left[-\omega(T)^{\frac{1}{2}}\right]+\omega(T)^{\frac{1}{2}} D_{\frac{k}{6}-1}\left[-\omega(T)^{\frac{1}{2}}\right] & =p D_{p-1}[z]-z D_{p}[z] \\
& =-D_{p+1}[z]
\end{aligned}
$$

Por lo tanto, la ecuación A.19 se escribe finalmente como

$$
\mathcal{F}_{k}(T)=\left(\frac{2 \epsilon}{k_{\mathrm{B}} T}\right)^{\frac{k}{12}} \Gamma\left[\frac{6-k}{6}\right] \exp \left[\frac{\epsilon}{2 k_{\mathrm{B}} T}\right] D_{\frac{k}{6}}\left[-\left(\frac{2 \epsilon}{k_{\mathrm{B}} T}\right)^{\frac{1}{2}}\right]
$$

\section{B.2. Evaluación analítica de integrales}

\section{B.2.1. Potencial esfericalizado SMP}

El potencial esfericalizado de un fluido PSM (sección 4.4.2 involucra el cálculo de la siguiente integral

$$
\begin{aligned}
\frac{1}{8 \pi}\left\langle\exp \left(-\frac{\Phi_{e l}}{k_{\mathrm{B}} T}\right)\right\rangle_{\Omega} & =\left\langle\sum_{m=0}^{\infty} \frac{1}{m !}\left(-\frac{\Phi_{i j}^{d d}}{k_{\mathrm{B}} T}\right)^{m} \sum_{n=0}^{\infty} \frac{1}{n !}\left(-\frac{\Phi_{i j}^{d i}}{k_{\mathrm{B}} T}\right)^{n}\right\rangle_{\Omega} \\
& =\sum_{m, n=0}^{\infty} \frac{1}{m ! n !}\left(-\frac{1}{k_{\mathrm{B}} T}\right)^{m+n}\left(\frac{\mu^{2}}{r^{3}}\right)^{m+n}\left(\frac{\alpha}{r^{3}}\right)^{n}\left\langle g_{i j}^{m} h_{i j}^{n}\right\rangle_{\Omega}
\end{aligned}
$$


donde las funciones $\Phi_{i j}^{d d}, \Phi_{i j}^{d i}$ y $\Phi_{e l}$ son los potenciales dados por las ecuaciones (4.8, 4.10, y 4.17), respectivamente. El cálculo de la integral angular se presenta por separado

$$
\begin{aligned}
&\left\langle g_{i j}^{m} h_{i j}^{n}\right\rangle_{\Omega}=\frac{1}{8 \pi} \int d \Omega\left(2 \cos \theta_{i} \cos \theta_{j}-\operatorname{sen} \theta_{i} \operatorname{sen} \theta_{j} \cos \varphi\right)^{m}\left(2+3 \cos ^{2} \theta_{i}+3 \cos ^{2} \theta_{j}\right)^{n} \\
&=\frac{1}{8 \pi} \int d \Omega \sum_{k=0}^{m}\left(\begin{array}{c}
m \\
k
\end{array}\right)\left(2 \cos \theta_{i} \cos \theta_{j}\right)^{m-k}\left(-\operatorname{sen} \theta_{i} \operatorname{sen} \theta_{j} \cos \varphi\right)^{k} \sum_{l=0}^{n}\left(\begin{array}{c}
n \\
l
\end{array}\right) 2^{n-l} 3^{l}\left(\cos ^{2} \theta_{i}+\cos ^{2} \theta_{j}\right)^{l} \\
&=\frac{1}{8 \pi} \int d \Omega \sum_{k=0}^{m}\left(\begin{array}{c}
m \\
k
\end{array}\right)\left(2 \cos \theta_{i} \cos \theta_{j}\right)^{m-k}\left(-\operatorname{sen} \theta_{i} \operatorname{sen} \theta_{j} \cos \varphi\right)^{k} \sum_{l=0}^{n}\left(\begin{array}{c}
n \\
l
\end{array}\right) 2^{n-l} 3^{l} \sum_{q=0}^{l}\left(\begin{array}{c}
l \\
q
\end{array}\right) \cos ^{2 q} \theta_{i} \cos ^{2 l-2 q} \theta_{j} \\
&=\frac{1}{8 \pi} \sum_{k=0}^{m} \sum_{l=0}^{n} \sum_{q=0}^{l}\left(\begin{array}{c}
m \\
k
\end{array}\right)\left(\begin{array}{c}
n \\
l
\end{array}\right)\left(\begin{array}{c}
l \\
q
\end{array}\right) 2^{m-k+n-l}(-1)^{k} 3^{l} \times \\
& \quad \times \int_{0}^{\pi} \cos ^{m-k+2 q} \theta_{i} \operatorname{sen}^{k+1} \theta_{i} d \theta_{i} \int_{0}^{\pi} \cos ^{m-k+2 l-2 q} \theta_{j} \operatorname{sen}^{k+1} \theta_{j} d \theta_{j} \int_{0}^{2 \pi} \cos ^{k} \varphi d \varphi
\end{aligned}
$$

donde se ha empleado de forma reiterada el teorema del binomio [21]. Obsérvese que la última integral se hace cero para valores impares de $k$; por lo que, $m$ debe ser par. Las integrales que aquí aparecen pueden expresarse usando la función beta $[10,20]$

$$
\mathrm{B}(x, y)=2 \int_{0}^{\pi / 2} \operatorname{sen}^{2 x-1} \varphi \cos ^{2 y-1} \varphi d \varphi
$$

lo que permite escribir finalmente

$$
\left\langle g_{i j}^{m} h_{i j}^{n}\right\rangle_{\Omega}=\sum_{k=0}^{m} \sum_{l=0}^{n} \sum_{q=0}^{l}\left(\begin{array}{l}
m \\
k
\end{array}\right)\left(\begin{array}{l}
n \\
l
\end{array}\right)\left(\begin{array}{l}
l \\
q
\end{array}\right) \frac{\mathrm{B}\left(\frac{k+2}{2}, \frac{m-k+2 q+1}{2}\right) \mathrm{B}\left(\frac{k+2}{2}, \frac{m-k+2 l-2 q+1}{2}\right) \mathrm{B}\left(\frac{k+1}{2}, \frac{1}{2}\right)}{2^{2-(m-k)-(n-l)} 3^{-l} \pi}
$$

recordando que sólo valores pares son permitidos para $k$ y $m$.

\section{B.2.2. Evaluación analítica de $\mathcal{D}_{k}(T)$ y $\mathcal{E}_{k}(T)$}

Los volúmenes de colisión, ecs. 3.11 3.12, pág. 33, contienen integrales que para resolverlas han sido reescritas de la siguiente manera:

$$
\begin{aligned}
\int_{0}^{1} \mathrm{e}^{-\Phi_{\mathrm{ANC}-2 \mathrm{~S}}^{*} / T^{*}} y^{2} d y= & \int_{y_{0}}^{1} \mathrm{e}^{-\Phi_{\mathrm{ANC}-2 \mathrm{~S}}^{*} / T^{*}} y^{2} d y=S_{\mathrm{R}}(1-a)\left[a^{2} \int_{0}^{1} e^{-\Phi_{\mathrm{LJ}}^{*} / T^{*}} d Y\right. \\
& \left.+a(1-a) \int_{0}^{1} \mathrm{e}^{-\Phi_{\mathrm{LJ}}^{*} / T^{*}} d Y^{2}+\frac{(1-a)^{2}}{3} \int_{0}^{1} \mathrm{e}^{-\Phi_{\mathrm{LJ}}^{*} / T^{*}} d Y^{3}\right] \\
= & S_{\mathrm{R}}(1-a)\left[a^{2} \mathcal{D}_{1}\left(T^{*}\right)+a(1-a) \mathcal{D}_{2}\left(T^{*}\right)+\frac{(1-a)^{2}}{3} \mathcal{D}_{3}\left(T^{*}\right)\right]
\end{aligned}
$$

donde $y_{0}=\left[1+S_{\mathrm{R}}\left(a^{3}-1\right)\right]^{1 / 3} \mathrm{y}$

$$
\begin{aligned}
\int_{1}^{\infty}\left(\mathrm{e}^{-\Phi_{\mathrm{ANC}-2 \mathrm{~S}}^{*} / T^{*}}-1\right) y^{2} d y= & S_{\mathrm{A}}(1-a)\left[a^{2} \int_{1}^{\infty}\left(\mathrm{e}^{-\Phi_{\mathrm{LJ}}^{*} / T^{*}}-1\right) d Y\right. \\
& +a(1-a) \int_{1}^{\infty}\left(\mathrm{e}^{-\Phi_{\mathrm{LJ}}^{*} / T^{*}}-1\right) d Y^{2} \\
& \left.+\frac{(1-a)^{2}}{3} \int_{1}^{\infty}\left(\mathrm{e}^{-\Phi_{\mathrm{LJ}}^{*} / T^{*}}-1\right) d Y^{3}\right] \\
= & S_{\mathrm{A}}(1-a)\left[a^{2} \mathcal{E}_{1}\left(T^{*}\right)+a(1-a) \mathcal{E}_{2}\left(T^{*}\right)+\frac{(1-a)^{2}}{3} \mathcal{E}_{3}\left(T^{*}\right)\right]
\end{aligned}
$$

donde, para ambos casos, se ha empleado el cambio de variable: $Y=\frac{\xi-a}{1-a}$, tal como se ha indicado en la sección 3.5 .2 . 
Analíticamente, y de manera similar a $\mathcal{F}_{k}(T)$, las integrales $\mathcal{D}_{k}(T)$ y $\mathcal{E}_{k}(T)$ pueden escribirse en términos de funciones gamma incompletas. La forma integral de las funciones gamma incompletas están dadas por $[10,20]$

$$
\gamma[a, x]=\int_{0}^{x} t^{a-1} \mathrm{e}^{-t} d t
$$

y

$$
\Gamma[a, x]=\int_{x}^{\infty} t^{a-1} \mathrm{e}^{-t} d t
$$

que se relacionan con la función gamma

$$
\Gamma[x]=\int_{0}^{\infty} t^{x-1} \mathrm{e}^{-t} d t
$$

de la siguiente manera

$$
\Gamma[\nu]=\Gamma[\nu, a]+\gamma[\nu, a] .
$$

Con ayuda de las definiciones anteriores, la integral $\mathcal{D}_{k}\left(T^{*}\right)$ se puede desarrollar de la siguiente forma

$$
\begin{aligned}
\mathcal{D}_{k}\left(T^{*}\right) & =\int_{0}^{1} \exp \left(-\frac{\Phi_{\mathrm{LJ}}^{*}}{T^{*}}\right) d Y^{k} \\
& =\int_{0}^{1} \exp \left(-\frac{Y^{-12}-2 Y^{-6}}{T^{*}}\right) d Y^{k} \\
& =\int_{0}^{1} \exp \left(-\frac{Y^{-12}}{T^{*}}\right) \exp \left(\frac{2 Y^{-6}}{T^{*}}\right) d Y^{k} \\
& =\int_{0}^{1} \exp \left(-\frac{Y^{-12}}{T^{*}}\right) \sum_{j=0}^{\infty} \frac{1}{j !}\left(\frac{2 Y^{-6}}{T^{*}}\right)^{j} d Y^{k} \quad \text { (Desarrollando la exponencial positiva) } \\
& =\sum_{j=0}^{\infty} \frac{2^{j}}{j ! T^{* j}} \int_{0}^{1} \exp \left(-\frac{Y^{-12}}{T^{*}}\right) Y^{-6 j} d Y^{k} \\
& \left.=\frac{k}{12} \sum_{j=0}^{\infty} \frac{2^{j}}{j !} T^{*-\frac{6 j+k}{12}} \int_{1 / T^{*}}^{\infty} \exp (-t) t^{\frac{6 j-k}{12}-1} d t \quad \text { (Empleando } t=Y^{-12} / T^{*}\right)
\end{aligned}
$$

y usando la ecuación (B.7) se tiene finalmente que

$$
\mathcal{D}_{k}\left(T^{*}\right)=\frac{k}{12} \sum_{j=0}^{\infty} \frac{2^{j}}{j !} \Gamma\left[\frac{6 j-k}{12}, \frac{1}{T^{*}}\right] T^{*-\frac{6 j+k}{12}} .
$$

Para la segunda integral se sigue un camino similar

$$
\begin{aligned}
\mathcal{E}_{k}\left(T^{*}\right) & =\int_{1}^{\infty}\left[\exp \left(-\frac{\Phi_{\mathrm{L} J}^{*}}{T^{*}}\right)-1\right] d Y^{k} \\
& =\int_{1}^{\infty}\left[\exp \left(-\frac{Y^{-12}}{T^{*}}\right) \exp \left(\frac{2 Y^{-6}}{T^{*}}\right)-1\right] d Y^{k} \\
& =\int_{1}^{\infty}\left[\exp \left(-\frac{Y^{-12}}{T^{*}}\right) \sum_{j=0}^{\infty} \frac{1}{j !}\left(\frac{2 Y^{-6}}{T^{*}}\right)^{j}-1\right] d Y^{k} \\
& =\sum_{j=0}^{\infty} \frac{2^{j}}{j ! T^{* j}} \int_{1}^{\infty} \exp \left(-\frac{Y^{-12}}{T^{*}}\right) Y^{-6 j} d Y^{k}-\int_{1}^{\infty} d Y^{k} \\
& =\frac{k}{12} \sum_{j=0}^{\infty} \frac{2^{j}}{j !} T^{*-\frac{6 j+k}{12}} \int_{0}^{1 / T^{*}} \exp (-t) t^{\frac{6 j-k}{12}-1} d t+1
\end{aligned}
$$


y con la ayuda de la forma integral (B.6) se tiene

$$
\mathcal{E}_{k}\left(T^{*}\right)=1+\frac{k}{12} \sum_{j=0}^{\infty} \frac{2^{j}}{j !} \gamma\left[\frac{6 j-k}{12}, \frac{1}{T^{*}}\right] T^{*-\frac{6 j+k}{12}} .
$$

Las expresiones (B.10) y (B.12) se emplean dentro de la teoría ANC en el cálculo analítico de los volúmenes de colisión de referencia, ecs. 3.23 y 3.24, pág. 34].

Nota. Debido a que

$$
\int_{0}^{1}\left[\exp \left(-\frac{\Phi_{\mathrm{LJ}}^{*}}{T^{*}}\right)-1\right] d Y^{k}=\mathcal{D}_{k}\left(T^{*}\right)-1
$$

entonces se cumple

$$
F_{k}\left(T^{*}\right)=1-\left[\mathcal{D}_{k}\left(T^{*}\right)+\mathcal{E}_{k}\left(T^{*}\right)\right]
$$

que relaciona las integrales (B.9) y (B.11) con A.17).

\section{B.2.3. Evaluación analítica de $h_{j}(T)$}

A continuación se muestra la resolución de la integral A.36 de $h_{j}(T)$. El potencial empleado es el LJ 12-6 dado por 2.13) por lo que dicha integral puede escribirse de la siguiente manera

$$
\begin{aligned}
h_{j}\left(T^{*}\right) & =-2 \pi \sigma^{3-6 j} \int_{0}^{\infty} z^{2-6 j} \exp \left[-\frac{4}{T^{*} z^{12}}\right] \exp \left[\frac{4}{T^{*} z^{6}}\right] d z \\
& =-2 \pi \sigma^{3-6 j} \sum_{l=0}^{\infty} \frac{1}{l !}\left(\frac{4}{T^{*}}\right)^{l} \int_{0}^{\infty} z^{2-6 j-6 l} \exp \left[-\frac{4}{T^{*} z^{12}}\right] d z \\
& =-\frac{\pi}{6} \sigma^{3-6 j} \sum_{l=0}^{\infty} \frac{1}{l !}\left(\frac{4}{T^{*}}\right)^{l} \int_{0}^{\infty} s^{-\frac{5-2 j-2 l}{4}} \exp \left[-\frac{4 s}{T^{*}}\right] d s \\
& =-\frac{\pi}{6} \sigma^{3-6 j} \sum_{l=0}^{\infty} \frac{1}{l !}\left(\frac{4}{T^{*}}\right)^{l}\left\{\left(\frac{4}{T^{*}}\right)^{\frac{1-2 j-2 l}{4}} \Gamma\left[\frac{2 j+2 l-1}{4}\right]\right\} \\
& =-\frac{\pi}{6} \sigma^{3-6 j}\left(\frac{4}{T^{*}}\right)^{\frac{1}{4}} \sum_{l=0}^{\infty} \frac{1}{l !}\left(\frac{4}{T^{*}}\right)^{\frac{l-j}{2}} \Gamma\left[\frac{2 j+2 l-1}{4}\right]
\end{aligned}
$$

donde se han empleado unidades reducidas $T^{*}=k_{\mathrm{B}} T / \epsilon, z=r / \sigma$ y se ha empleado el cambio de variable intermedio $s=z^{-12}$. Al igual que en otros casos al final se ha empleado la definición integral de la función gamma $[10,20]$, ec. B.8. 


\section{B.3. Ajustes numéricos}

\section{B.3.1. Escalamiento potencial PSM: Coeficientes para $F_{\epsilon}$ y $F_{\delta}$}

A continuación se presenta la forma de las funciones dependientes de la temperatura $\mathcal{A}_{i}\left(T^{*}\right)$ (caso SM) y $\mathcal{B}_{i}\left(T^{*}\right)$ (caso PSM) que aparecen en las ecuaciones 4.37, 4.38, pág. 57):

$$
\begin{aligned}
& \mathcal{A}_{i}\left(T^{*}\right)=T^{* p}\left(\mathcal{A}_{i 1} T^{*}+\mathcal{A}_{i 2} T^{* 3 / 4}+\mathcal{A}_{i 3} T^{* 1 / 2}\right) \\
& \mathcal{B}_{i}\left(T^{*}\right)=T^{* q} \frac{\mathcal{B}_{i 1} T^{* 3}+\mathcal{B}_{i 2} T^{* 2}+\mathcal{B}_{i 3} T^{*}+\mathcal{B}_{i 4}}{\mathcal{B}_{i 5} T^{* 3}+\mathcal{B}_{i 6} T^{* 2}+\mathcal{B}_{i 7} T^{*}+\mathcal{B}_{i 8}}
\end{aligned}
$$

con

\begin{tabular}{|c|c|c|c|}
\hline${ }_{i} \longrightarrow j$ & 1 & 2 & 3 \\
\hline 1 & -0.044057 & 0.393265 & 0.16051864 \\
\hline 2 & 0.005260116 & -0.01516189 & 0.01217602 \\
\hline 3 & -0.12202235 & 0.1902944 & -0.0543076 \\
\hline 4 & 0 & 0.2807762 & -0.13674715 \\
\hline
\end{tabular}

$$
p=\left\{\begin{array}{cc}
0, & i=1,3,4,6 \\
2, & i=2,5
\end{array}, \mathrm{y} \quad q= \begin{cases}1, & i=1,3 \\
2, & i=2,4\end{cases}\right.
$$

Tabla B.1: Coeficientes $\mathcal{A}_{i j}$.

\begin{tabular}{|c|c|c|c|c|c|c|c|c|}
\hline$i$ & 1 & 2 & 3 & 4 & 5 & 6 & 7 & 8 \\
\hline 1 & 1 & -1.053332 & 0.467631 & 0 & 1 & -0.229426 & 0.195709 & 0 \\
\hline 2 & 0 & 0.6562554 & 0.0129494 & 0 & 1 & -0.068386 & 0.23084 & 0 \\
\hline 3 & -0.122634 & -0.33913 & -0.271577 & 0 & 1 & 4.9155 & 1.83045 & -0.1306995 \\
\hline 4 & 0.008203 & -0.0121586 & 0.00850234 & 0 & 1 & -0.838778 & 0.6513865 & -0.0429287 \\
\hline
\end{tabular}

Los coeficientes de estas funciones están dados en las tablas B.1 y B.2 y son válidos en los intervalos: $T^{*} \in[0.3,10.0], \mu^{*} \in[0.0,2.0]$ y $\alpha^{*} \in[0.0,0.1]$.

Tabla B.2: Coeficientes $\mathcal{B}_{i j}$. 


\section{Apéndice $\mathrm{C}$}

\section{Cuerpos no esféricos}

\section{C.1. Dinámica de cuerpo rígido}

\section{Ángulos de Euler}

La orientación de un cuerpo rígido puede especificarse completamente considerando un sistema de coordenadas fijo a él. Es necesario especificar la relación entre un sistema de ejes fijos en el espacio y uno fijo en el cuerpo. Se puede construir la transformación entre estos dos sistemas con tres rotaciones virtuales sucesivas del cuerpo rígido; de esta manera se introducen los ángulos de Euler [22]. Los ángulos de Euler $\varphi, \theta$ y $\psi$ especifican la orientación del sistema fijo al cuerpo relativo con respecto al sistema fijo en el espacio, y por lo tanto, actúan como tres coordenadas generalizadas. La transformación del sistema del laboratorio al sistema del cuerpo puede lograrse a través de la matriz de transformación $A$, dada por

$$
A=\left(\begin{array}{ccc}
\cos \phi \cos \psi-\operatorname{sen} \phi \cos \theta \operatorname{sen} \psi & \operatorname{sen} \phi \cos \psi+\cos \phi \cos \theta \operatorname{sen} \psi & \operatorname{sen} \theta \operatorname{sen} \psi \\
-\cos \phi \operatorname{sen} \psi-\operatorname{sen} \phi \cos \theta \cos \psi & -\operatorname{sen} \phi \operatorname{sen} \psi+\cos \phi \cos \theta \cos \psi & \operatorname{sen} \theta \cos \psi \\
\operatorname{sen} \phi \operatorname{sen} \theta & -\cos \phi \operatorname{sen} \theta & \cos \theta
\end{array}\right) .
$$

Es una ventaja tomar como ejes del sistema del cuerpo los ejes principales; es decir, los ejes para los cuales el tensor de inercia I es diagonal. Adicionalmente, el eje de simetría es uno de los ejes principales y será escogido como eje $z$ del sistema de coordenadas fijo al cuerpo. Para el movimiento rotacional sobre un punto fijo, el uso de la segunda ley de Newton nos lleva a un conjunto de ecuaciones conocidas como las ecuaciones de Euler [23]

$$
\left(\frac{d \mathbf{L}}{d t}\right)_{e}=\left(\frac{d \mathbf{L}}{d t}\right)_{c}+\boldsymbol{\omega} \times \mathbf{L} \quad \Rightarrow \quad \begin{aligned}
I_{1} \dot{\omega}_{1}-\omega_{2} \omega_{3}\left(I_{2}-I_{3}\right) & =N_{1} \\
I_{2} \dot{\omega}_{2}-\omega_{3} \omega_{1}\left(I_{3}-I_{1}\right) & =N_{2} \\
I_{3} \dot{\omega}_{3}-\omega_{1} \omega_{2}\left(I_{1}-I_{2}\right) & =N_{3}
\end{aligned}
$$

donde $\mathbf{L}$ es el momento angular del cuerpo, y $\boldsymbol{\omega}$ su velocidad angular.

Los subíndices $c$ y $e$ indican el marco con respecto al cuerpo e independiente de él, respectivamente y los $\omega_{i}$ y $N_{i}$, medidos en el sistema del cuerpo. El cambio en los ángulos de Euler está dado por

$$
\dot{\phi}=-\omega_{1} \frac{\operatorname{sen} \phi \cos \theta}{\operatorname{sen} \theta}+\omega_{2} \frac{\cos \phi \cos \theta}{\operatorname{sen} \theta}+\omega_{3}, \quad \dot{\theta}=\omega_{1} \cos \phi+\omega_{2} \operatorname{sen} \phi, \quad \dot{\psi}=\omega_{1} \frac{\operatorname{sen} \phi}{\operatorname{sen} \theta}-\omega_{2} \frac{\cos \phi}{\operatorname{sen} \theta}
$$

donde las velocidades angulares son medidas en el sistema del espacio. 


\section{Ecuaciones de movimiento para sistemas lineales}

Sea $\mathbf{N}$ la torca sobre una partícula dada por

$$
\mathbf{N}=\mathbf{s} \times \mathbf{G}
$$

donde $\mathbf{s}$ es un vector unitario que indica su orientación y $\mathbf{G}$ el campo aplicado a ella que la rota. En el caso lineal el momento angular es simplemente $\mathbf{L}=I \boldsymbol{\omega}$ donde $I$ es el momento de inercia y $\boldsymbol{\omega}$ es la velocidad angular. Las ecuaciones de movimiento para esta partícula son

$$
\begin{aligned}
I \dot{\omega} & =\mathbf{s} \times \mathbf{G} \\
\dot{\mathbf{s}} & =\omega \times \mathbf{s}
\end{aligned}
$$

y de este par ecuaciones de primer orden se puede obtener una ecuación de segundo orden al eliminar a $\omega$

$$
\begin{aligned}
\ddot{\mathbf{s}} & =\dot{\boldsymbol{\omega}} \times \mathbf{s}+\boldsymbol{\omega} \times \dot{\mathbf{s}}=I^{-1}(\mathbf{s} \times \mathbf{G})+\boldsymbol{\omega} \times(\boldsymbol{\omega} \times \mathbf{s}) \\
& =I^{-1} \mathbf{G}-\left(I^{-1}(\mathbf{s} \cdot \mathbf{G})+\dot{\mathbf{s}}^{2}\right) \mathbf{s}
\end{aligned}
$$

donde se ha usado $\boldsymbol{\omega} \cdot \mathbf{s}=0 \mathrm{y} \dot{\mathbf{s}}^{2}=\omega^{2}$.

\section{C.2. Teoría básica de cuerpos convexos.}

Un cuerpo convexo puede caracterizarse por tres medidas fundamentales [15] $V, A$, y $M$ las cuales identifican su volumen, su área superficial y el radio medio de curvatura. Éste último parámetro se encuentra mediante la siguiente integral

$$
M=\frac{1}{2} \iint\left(\frac{1}{R_{1}}+\frac{1}{R_{2}}\right) d A
$$

donde $R_{1}$ y $R_{2}$ son los radios principales de curvatura.

Dichas medidas fundamentales generalmente se escriben en función del ancho del cuerpo $h$ y de su elongación $\xi$, la cual está definida como la razón entre la zona más ancha y la más angosta, $\xi=L / h$. En la tabla C.1 se muestran algunos ejemplos que serán empleados en ese trabajo junto con sus medidas fundamentales [7,24,25]. Las etiquetas manejadas para estos cuerpos se dan en el cuerpo principal de la tesis, sección 5.5

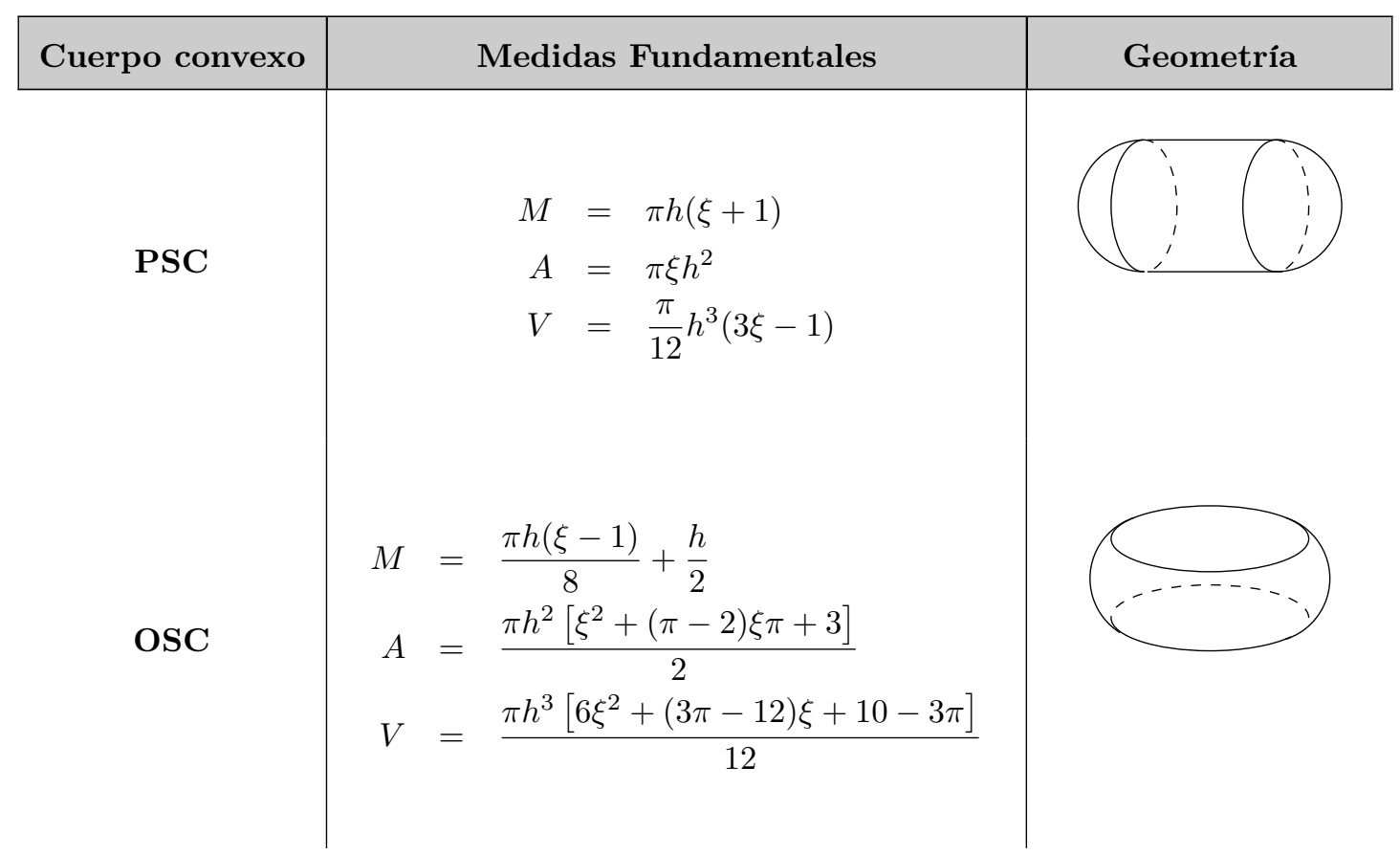


Tabla C.1: Continuación

PEL

$$
\begin{aligned}
M & =\frac{h}{4}\left[\xi+\frac{\ln \left(\left(\xi+\sqrt{\xi^{2}-1}\right)\right.}{\sqrt{\xi^{2}-1}}\right] \\
A & =\frac{\pi h^{2}}{2}\left[1+\frac{\xi^{2} \cos ^{-1} \xi^{-1}}{\sqrt{\xi^{2}-1}}\right]
\end{aligned}
$$$$
V=\frac{\pi}{6} \xi h^{3}
$$

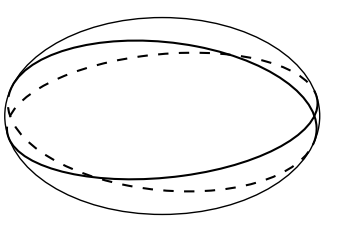

OEL

$$
\begin{aligned}
M & =\frac{h}{4}\left[\frac{1}{\xi}+\frac{\xi \cos ^{-1}(1 / \xi)}{\sqrt{\xi^{2}-1}}\right] \\
A & =\frac{\pi h^{2}}{2}\left[1+\frac{1}{\xi \sqrt{\xi^{2}-1}}\right] \ln \left[\xi+\sqrt{\xi^{2}-1}\right]
\end{aligned}
$$

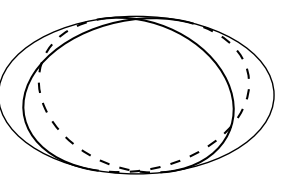

$V=\frac{\pi}{6} \xi^{-1} h^{3}$

DC

$$
\begin{aligned}
M & =\frac{h}{4}\left(\xi+\xi^{-1}\right) \\
A & =\frac{\pi h^{2}}{2}\left(\xi+\xi^{-1}\right) \\
V & =\frac{\pi h^{3}}{12}\left(\xi+\xi^{-1}\right)
\end{aligned}
$$

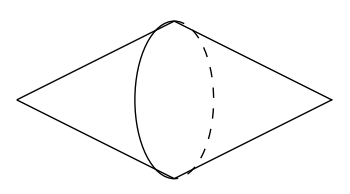

EC

$$
\begin{aligned}
M & =\frac{\pi h}{2}\left(2 \xi+1+\frac{1}{2 \xi-1}\right) \\
A & =\frac{\pi h^{2}}{4}\left(2 \xi+1+\frac{1}{2 \xi-1}\right) \\
V & =\frac{\pi h^{3}}{24}\left(2 \xi+1+\frac{1}{2 \xi-1}\right)
\end{aligned}
$$

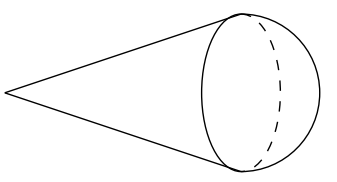

Tabla C.1: Cuerpos convexos usados en el cálculo del segundo coeficiente virial para interacciones tipo CSW y CANC, y sus medidas fundamentales $M, A$, y $V$. Ancho del cuerpo $(h)$ y razón ancho/largo $(\xi)$. Las medidas fundamentales tienen unidades de distancia $(M)$, área $(A)$ y volumen $(V)$. 


\begin{tabular}{|c|c|}
\hline CB & $\left\langle V_{h+h}\right\rangle / \frac{4 \pi}{3} h^{3}$ \\
\hline PSC & $1+\frac{3}{2} L^{*}+\frac{3}{8} L^{* 2}$ \\
\hline OSC & $1+\frac{3}{4} \pi L^{*}+\frac{3}{32}\left(8+\pi^{2}\right) L^{* 2}+\frac{3}{32} \pi L^{* 3}$ \\
\hline PEL & $\frac{\xi_{h}}{4}+\frac{3}{16}\left[\left(1+\frac{\xi_{h}^{2} \cos ^{-1} \xi_{h}^{-1}}{\sqrt{\xi_{h}^{2}-1}}\right)\right]\left[\xi_{h}+\frac{\ln \left(\xi_{h}+\sqrt{\xi_{h}^{2}-1}\right)}{\sqrt{\xi_{h}^{2}-1}}\right]$ \\
\hline OEL & $\frac{\xi_{h}^{2}}{4}+\frac{3 \xi_{h}^{2}}{16\left(\xi_{h}^{2}-1\right)}\left[\xi_{h}^{-1} \sqrt{\xi_{h}^{2}-1}+\xi_{h} \cos ^{-1} \xi_{h}^{-1}\right]\left[\xi_{h} \sqrt{\xi_{h}^{2}-1}+\ln \left(\xi_{h}+\sqrt{\xi_{h}^{2}-1}\right)\right.$ \\
\hline EC & $\frac{3 \xi_{h}^{4}+2 \xi_{h}^{3}-\xi_{h}^{2}}{4\left(1-2 \xi_{h}\right)^{2}}$ \\
\hline DC & $\frac{3+2 \xi_{h}+6 \xi_{h}^{2}+2 \xi_{h}^{3}+3 \xi_{h}^{4}}{16 \xi_{h}^{2}}$ \\
\hline
\end{tabular}

Tabla C.2: Volúmenes $\left\langle V_{h+h}\right\rangle$ de algunos de los cuerpos empleados en el presente estudio. Para los dos primeros $\operatorname{casos} L^{*}=\xi_{h}-1$.

\section{Cuerpo convexo paralelo}

Considérense dos cuerpos convexos tal que uno cubra por completo al otro y que la distancia $\rho$ entre sus respectivas superficies se mantenga constante, ver figura C.1. El CB central, de ancho $h_{1}$ y largo $L_{1}$, tiene medidas fundamentales $V_{1}, A_{1}$ y $M_{1}$ y se le denotará como cuerpo 1 con centro de masa en $O_{1}$. Las medidas fundamentales del cuerpo externo (cuerpo paralelo a 1) de ancho $H=h_{1}+2 \rho$ y elongación $L_{H}$ pueden ser escritas en términos de las respectivas medidas del cuerpo central

$$
\begin{aligned}
V_{1+\rho} & =V_{1}+A_{1} \rho+M_{1} \rho^{2}+\frac{4}{3} \pi \rho^{3} \\
A_{1+\rho} & =A_{1}+2 M_{1} \rho+4 \pi \rho^{2} \\
M_{1+\rho} & =M_{1}+4 \pi \rho
\end{aligned}
$$

y son conocidas como relaciones de Steiner [16]; a este último cuerpo se le ha denotado como $1+\rho$, según la notación acostumbrada en teoría de cuerpos convexos [24].

\section{Covolumen de un cuerpo convexo}

Manteniendo fijo el cuerpo $1+\rho$ arriba definido, ahora se desplaza alrededor de él un cuerpo 2, caracterizado por $M_{2}, A_{2}$ y $V_{2}$, manteniendo fija su orientación, figura C.1. El centro de gravedad, $\mathrm{O}_{2}$ de este nuevo CB forma la superficie de otro cuerpo convexo que denotaremos por $1+\rho+2$. Si esta operación se repite sobre todas las posibles orientaciones de 2 , y se promedian angularmente, se obtiene el cuerpo convexo $\langle 1+\rho+2\rangle$, con medidas 
fundamentales promediq ${ }^{1}$

$$
\begin{aligned}
\left\langle V_{1+\rho+2}\right\rangle=V_{1}+V_{2}+ & \frac{1}{4 \pi}\left(M_{1} A_{2}+M_{2} A_{1}\right) \\
& +\left(A_{1}+A_{2}+\frac{1}{2} M_{1} M_{2}\right) \rho+\left(M_{1}+M_{2}\right) \rho^{2}+\frac{4}{3} \pi \rho^{3} \\
\left\langle A_{1+\rho+2}\right\rangle= & A_{1}+A_{2}+\frac{1}{2 \pi} M_{1} M_{2}+2\left(M_{1}+M_{2}\right) \rho+\pi \rho^{2} \\
\left\langle M_{1+\rho+2}\right\rangle= & M_{1}+M_{2}+4 \pi \rho
\end{aligned}
$$

escrito en términos de las medidas fundamentales de los dos cuerpos rígidos y donde \langle\rangle indica el valor medio sobre todas las orientaciones.

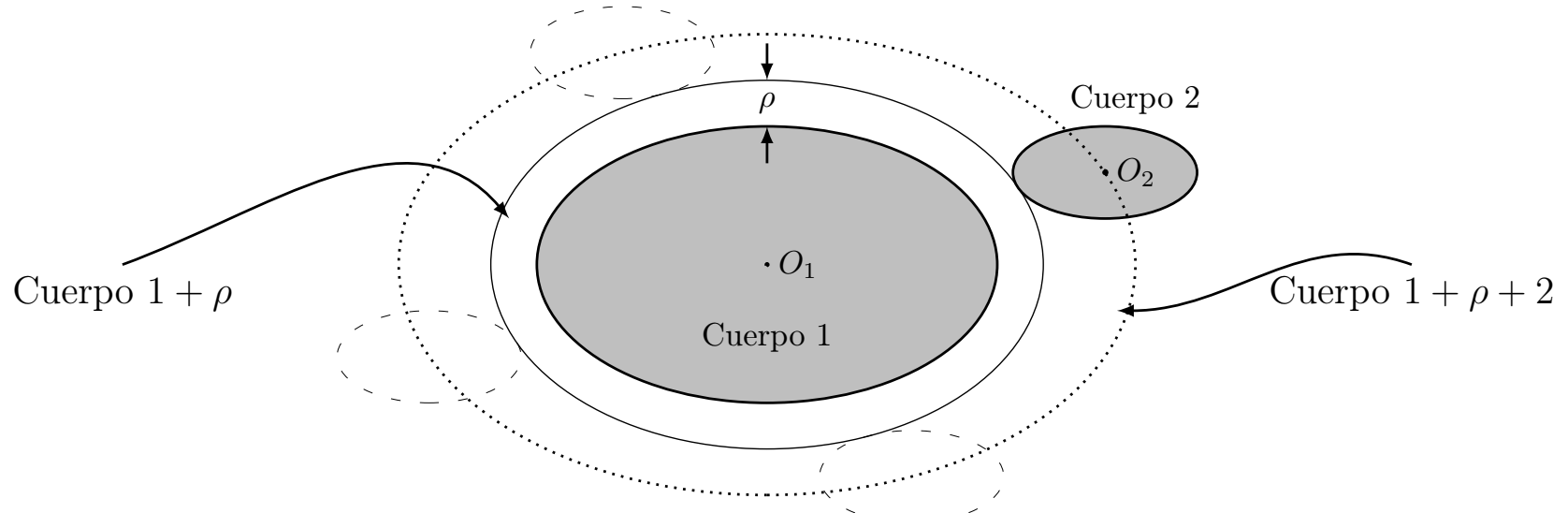

Figura C.1: Cuerpo $1+\rho+2$ (línea punteada) formado por dos cuerpos convexos 1 y 2 cuyas superficies siempre se encuentran separadas una distancia $\rho$.

Para el caso en que los nódulos 1 y 2 se tocan $(\rho=0)$, el volumen obtenido por C.10 se le conoce comúnmente como covolumen. Esta cantidad corresponde, cuando se aplica al modelado de gases, precisamente al volumen ocupado por dos moléculas el cual debe excluirse del total sobre el que se pueden mover el resto de ellas.

\section{Referencias}

[1] W. Greiner, L. Neise, and H. Stocker. Thermodynamics and Statistical Mechanics. Springer, 1997.

[2] R. K. Pathria. Statistical Mechanics. BH, Oxford, 2001.

[3] A. Isihara. Statistical Physics. Academic Press Inc., New York, USA, 1971.

[4] J. E. Lennard-Jones. Proc. Phys. Soc., 43:461-482, 1931.

[5] T. Kihara. J. Phys. Soc. Japan, 6(5):289-296, 1951.

[6] T. Kihara. Rev. Modern Phys., 25(4):831-843, 1953.

[7] W. Witschel. Int. J. Thermophys., 11(6):1075-1085, 1990.

[8] P. Vargas, E. Muñoz, and L. Rodriguez. Phys. A, 290:92-100, 2000.

\footnotetext{
${ }^{1}$ Método establecido por separado por Isihara y por Hadwiger [26].
} 
[9] A. J. M. Garrett. J. Phys. A: Math. Gen., 13:379-380, 1980.

[10] I. S. Gradshteyn and I. M. Ryzhik. Table of Integrals, series, and products. Elsevier, 2007.

[11] A. D. Buckingham and J. A. Pople. Trans. Far. Soc., 51:1173-1179, 1955.

[12] W. H. Stockmayer. J. Chem. Phys., 9:398-402, 1941.

[13] J. S. Rowlinson. Trans. Far. Soc., 45:974, 1949.

[14] S. C. Saxena and K. M. Joshi. Phys. fluids, 5(10):1217-1222, 1962.

[15] T. Kihara. Intermolecular Forces. John Wiley and Sons, New York, 1978.

[16] T. Kihara and K. Miyoshi. J. Stat. Phys., 13(4), 1975.

[17] J. Bartke and R. Hentschke. Phys. Rev. E, 75:061503, 2007.

[18] R. B. Bird, E. L. Spotz, and J. O. Hirschfelder. J. Chem. Phys., 18(10):1395-1402, 1950.

[19] J. S. Rowlinson. J. Chem. Phys., 19:7, 1951.

[20] M. Abramowitz and I. A. Stegun. Handbook of Mathematical Functions with Formulas, Graphs, and Mathematical Tables. Dover, New York, 1972.

[21] A. D. Polyanin and A. V. Manzhirov. Handbook of mathematics for engineers and scientists. Chapman \& Hall/CRC, Taylor \& Francis Group, 2007.

[22] H. Goldstein. Classsical mechanics. Reverté S. A., 2000.

[23] S. T. Thornton and J. B. Marion. Classsical dynamics of particles and systems. Thomson. Brooks/Cole, 2004 .

[24] C. G. Gray, K. E. Gubbins, and C. G. Joslin. Theory of Molecular Fluids Volume 2: Applications. Oxford University Press, Oxford, 2011.

[25] T. Kihara, K. Yamazaki, M. S. Jhon, and U. R. Kim. Chem. Phys. Lett., 9(1):62-64, 1971.

[26] T. Kihara. Prog. Theor. Phys. Supplement, (40), 1967. 